\title{
Klystron Switching Power Supplies for the International Linear Collider
}

Andrea Fraioli

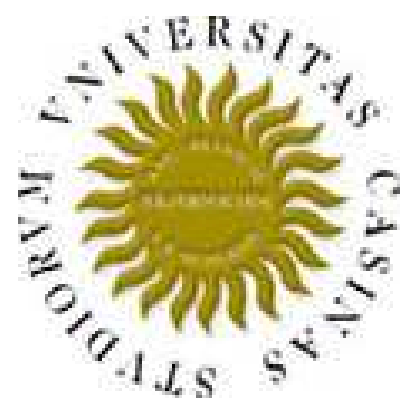

Master's Degree Dissertation

December 2009 


\title{
DISSERTATION ABSTRACT
}

\section{Klystron Switching Power Supplies For the International Linear Collider}

\author{
dott. Andrea Fraioli
}

Supervisor

Prof. Giovanni Busatto
Supervisor

Prof. Carmine E. Pagliarone

Electrical Engineering Master's Degree

At the

UNIVERSITY OF CASSINO

FACULTY OF ENGINEERING

December 2009

The International Linear Collider is a majestic High Energy Physics particle accelerator that will give physicists a new cosmic doorway to explore energy regimes beyond the reach of today's accelerators. ILC will complement the Large Hadron Collider (LHC), a proton-proton collider at the European Center for Nuclear Research (CERN) in Geneva, Switzerland, by producing electron-positron collisions at center of mass energy of about $500 \mathrm{GeV}$. In particular, the subject of this dissertation is the $R \& D$ for a solid state Marx Modulator and relative switching power supply for the International Linear Collider Main LINAC Radio Frequency stations. 


\section{Acknowledgements}

I would like to express my gratitude to Prof. Carmine Elvezio Pagliarone. His continuous supervision during my work at Fermi National Accelerator Laboratory (Fermilab) and before has been for me very important and intellectually a pleasure. He guided me through all different aspects of Particle Accelerator, Collider Physics and in particular on our intense $R \& D$ on a Solid State Marx Modulator for the ILC Klystron Modulators.

I would like to extend my gratitude to Prof. Giovanni Busatto for his supervision during my work at University of Cassino. His knowledge of power electronics devices has guided me during the Switch in Line Converter project.

Besides I want express my gratitude to the NTA-ILC Pisa Group Leader Dott. Franco Bedeschi, for supporting us in this R\&D activity, and then to Prof. Giovanni Maria Piacentino, the senior Cassino University physicist for the support and collaboration in the Cassino Group. At the end a special thanks goes to Fermi National Accelerator Laboratory for technical support during this research activity. 


\section{Dedication}

A mia Madre ed a mio Padre, per aver dedicato tutto il loro amore e tutti i loro sacrific quotidiani a me e a mio Fratello, dedico questa tesi quale frutto di un intenso lavoro intellettuale prolifico di soddisfazioni. 


\section{Table of Contents}

$\begin{array}{ll}\text { Abstract } & \text { ii } \\ \text { Acknowledgements } & \text { iii } \\ \text { Dedication } & \text { iv } \\ \text { Index } & \text { v } \\ \text { List of Tables } & \text { viii } \\ \text { List of Figures } & \text { ix }\end{array}$ 


\section{INDEX}

\section{Physics motivations for the International Linear Collider}

1.1. Introduction

1.2. Higgs Boson Physics

1.3. Dark Matter \& Extra Dimension

\section{The ILC accelerator Design}

2.1. Electron and positron sources 8

2.2. Service Rings 10

2.2.1. Damping Rings 12

2.2.2. Ring to Main Linac 12

2.3. Main Linac 15

2.4. The ILC Accelerating system 16

$\begin{array}{ll}\text { 2.4.1. Superconducting cavities } & 17\end{array}$

$\begin{array}{ll}\text { 2.4.2. RF distribution system } & 17\end{array}$

$\begin{array}{ll}\text { 2.4.3. Klystron } & 19\end{array}$

2.4.4. Klystron Modulator 21

2.4.5. The cryogenic systems 24

2.5. Estimate for construction of ILC 26

\section{Klystron Modulator Power Supplies}

3.1. Modulator charging power supply design requirements 35

3.2. Modulator power supply design analysis 37

3.2.1. TESLA design 37

3.2.2. A Russian design for TESLA 39

3.2.3. Fermilab Power Supply 42

3.2.4. SCR bridge in series with a diode rectifier 43

3.2.4.1. SCR bridge with single phase control $\quad 44$

3.2.4.2. Digital regulation 46

3.2.5. Series connection of buck converters 50

3.3. The Buck converter solution 51

3.3.1. Buck converter schematic and design requirements 51

3.3.2. Circuit simulation and analysis $\quad 52$

3.3.3. Main switch control for constant power 53

3.3.4. Buck converter waveforms 58

3.4. The Switch In Line Converter 59

3.4.1. Operation Principle 59

3.4.2. $30 \mathrm{~kW} \mathrm{SILC} \mathrm{Project} \quad 61$

3.4.2.1. $\quad$ SILC design requirements and Project 62

$\begin{array}{lll}\text { 3.4.2.2. } & \text { Circuit simulation and analysis } & 64\end{array}$

$\begin{array}{lll}\text { 3.4.2.3. Control signals of switches } & 64\end{array}$ 
3.4.2.4. SILC waveforms $\quad 69$

$\begin{array}{ll}\text { 3.4.2.5. Soft switching commutation } & 69\end{array}$

$\begin{array}{lll}\text { 3.4.2.5.1. Snubbers circuits } & 69\end{array}$

3.4.2.6. Select of SILC's switches $\quad 75$

3.4.2.6.1. Insulated Gate Bipolar Transistors (IGBTs) 76

3.4.2.6.2. Choose of snubber capacity and switching commutations 76

\section{A Solid State Marx Modulator for ILC}

$\begin{array}{ll}\text { 4.1. Introduction } & 79\end{array}$

4.2. The Fermilab Bouncer Modulator $\quad 79$

4.2.1. Operation principle $\quad 81$

4.2.2. The Bouncer Modulator key components and costs 81

4.3. The Marx Generator Concept 83

$\begin{array}{ll}\text { 4.3.1. Introduction } & 83\end{array}$

$\begin{array}{ll}\text { 4.3.2. The classical layout } & 84\end{array}$

4.3.3. Solid State Marx Generator 86

4.4. $\mathrm{A} \mathrm{SM}^{2}$ Solid State Marx Modulator for ILC $\quad 87$

4.4.1. The $\mathrm{SM}^{2}$ fundamental cell design $\quad 89$

4.4.2. The $\mathrm{SM}^{2}$ Main Marx generator 90

4.4.2.1. The Main Marx Layout and Design Requirements 90

4.4.2.2. Stray Capacitance Charging Losses $\quad 92$

$\begin{array}{ll}\text { 4.4.2.3. Main Marx output response } & 92\end{array}$

4.4.3. The Flattener circuit 94

4.4.4. The $\mathrm{SM}^{2}$ Switching Trigger System 96

4.5. Solid State Marx Modulator Single Failure Risks 98

4.6. $\mathrm{SM}^{2}$ Modulator circuit simulations and analysis 99

$\begin{array}{ll}\text { 4.7. Conclusions } & 100\end{array}$

\section{Appendix}

A. The synchrotron radiation energy loss 104

$\begin{array}{ll}\text { B. The concept of luminosity } & 109\end{array}$

\section{Bibliography}




\section{LIST OF TABLES}

2.01 Electrons Source System parameters 11

2.02 Nominal Positron Source parameters. 11

2.03 Positron Damping Ring parameters; the Electron Damping Ring is identical 13 except for a smaller injected emittance.

2.04 Basic beam parameters of the RTML. 15

2.05 Nominal beam parameters in the ILC Main Linac. 16

2.06 ILC 9-cell Superconducting Cavity layout parameters. 18

2.07 Properties of high-RRR (residual resistivity ratio) Niobium suitable for use in ILC 18 Cavities.

2.08 RF Unit parameters. $\quad 20$

2.09 10MW MBK parameters. 22

2.10 Modulator Specifications \& Requirements Assuming Klystron $\mu \mathrm{P}=3.38, \quad 25$ Effy $=65 \%$.

2.11 Superconducting RF modules in the ILC, excluding the two 6-cavity energy 27 compressor cryomodules located in the electron and positron LTRs.

2.12 Possible division of responsibilities for the 3 sample sites (ILC Units). 31

2.13 Distribution of the ILC Value Estimate by area system and common infrastructure, 32 in ILC Units. The estimate for the experimental detectors for particle physics is not included .

2.14 Explicit labor, which may be supplied by collaborating laboratories or institutions, listed by Global, Technical, and some Area-specific Systems.

2.15 Composition of the management structure at ILC. 


\section{LIST OF FIGURES}

1.01 Feynman diagrams for Higgsstrahlung (top) and WW fusion (bottom). Right: cross sections of the two processes as function of the center of mass energy for Higgs masses of 200 and $320 \mathrm{GeV} / \mathrm{c}^{2}$.

$1.02 \mathrm{SM}$ and MSSM predictions for $\mathrm{g}_{\mathrm{ttH}}$ versus $\mathrm{g}_{\mathrm{WWH}}$ and expected precision of the corresponding LHC and ILC measurements

1.03 Reconstructed mass sum and difference for $\mathrm{HA} \rightarrow 4 \mathrm{~b}$ for masses of $\mathrm{m}_{\mathrm{H}}=250$ $\mathrm{GeV} / \mathrm{c}^{2}$ and $\mathrm{m}_{\mathrm{A}}=300 \mathrm{GeV} / \mathrm{c}^{2}$ at a center of mass energy of $800 \mathrm{GeV} / \mathrm{c}^{2}$.

1.04 Left: Production mechanism for $\mathrm{e}^{+} \mathrm{e}^{-} \rightarrow \gamma \mathrm{G}$, the graviton escapes into extra dimensions. Right: The cross section of this process as a function of the center of mass energy for different numbers of extra dimensions. The points with error bars indicate the precision of the ILC measurements.

1.05 Dark matter relic density versus WIMP mass in the LCC1 SUSY scenario. Possible parameter choices are indicated as black dots, which are compared to the sensitivity of present and future measurements from satellite and accelerator based experiments.

2.01 A schematic layout of the International Linear Collider

2.02 Cutaway view of the linac dual-tunnel configuration. On the left you can see the Main Linac tunnel, on the right the service tunnel hosting the klystrons, the klystron modulators and switching power supplies.

2.03 Schematic view of the polarized Electron Source.

2.04 Overall layout of the Positron Source.

2.05 Dynamic aperture of ILC Damping Ring (without field or alignment error) for relative momentum errors of $-1 \%, 0 \%$ and $1 \%$ at $\mathrm{x}=44 \mathrm{~m}$ and $\mathrm{y}=18 \mathrm{~m}$. The tick green line represents the size of the injected positron beam.

2.06 Schematic of RTML.

2.07 9-cells Superconducting RF Cavity.

$2.08 \mathrm{RF}$ unit diagram showing the basic waveguide distribution layout between the klystron and 26 cavities in three cryomodules.

2.09 Waveguide circuit from tap-off hybrid to coupler input, showing the various components (except for the directional coupler).

2.10 Toshiba 3736 Multi-Beam Klystron.

2.11 a) CPI VKL-8301, b) Thales TH 1801, c) Toshiba MBK E3736.

2.12 Test results for: a) CPI VKL-8301 at reduced pulse width; b) Toshiba MBK E3736 at full spec pulse width; c) Thales TH1801 at reduced pulse width. 
2.13 a) Capacitor Stack, b) Dual IGBT Switch, c) Bouncer Choke, d) Pulse transformer. $\quad 24$

2.14 Damping Ring 1.2 MW RF station (1 of 20). 25

2.15 The overall layout concept for the cryogenic systems. 26

2.16 Cooling scheme of a cryo-string. 28

2.17 Two-phase helium flow for level and for sloped systems. 29

2.18 Distribution of the ILC value estimate by area system and common infrastructure, 30 in ILC Units. The estimate for the experimental detectors for particle physics is not included.

2.19 Explicit labor, which may be supplied by collaborating laboratories or institutions listed by Global, Technical, and some Area-specific Systems.

3.01 Schematic of the series resonance sine converter. 36

3.02 Equivalent circuit of the power supply. 36

3.03 Series resonance sine converter with only one capacitor 36

3.04 Voltage and current function of the half bridge. 37

3.05 Voltage and current waveform of power supply. 38

3.06 Simplified circuit diagram of a charge device with an electron protection. 39

3.07 Fermilab DC power supply $10.6 \mathrm{kV} @ 14 \mathrm{~A} . \quad 41$

3.08 PS Voltage / Current and Capacitor Voltage. 42

3.09 Series connection of a diode and a SCR bridge. 43

3.10 Voltage curve form of the main capacitor bank in the modulator. 43

3.11 Voltage curve of series connection of diode and SCR bridge. 44

3.12 Real and reactive power in dependence of the phase angle $\alpha$. 45

3.13 Power diagram of a series connection of a diode and SCR bride $\alpha(\mathrm{P})$. 46

3.14 Simplified modulator circuit. $\quad 46$

3.15 Voltage curve of the main capacitor. $\quad 47$

3.16 Stored reference charging curve of the capacitor. $\quad 47$

3.17 Input power of the modulator. $\quad 47$

3.18 RAM curves of the capacitor reference curves. 48 
3.19 Linearized and scaled reference curve and monitored curve. 48

3.20 Output signal of the regulation. $\quad 48$

3.21 Block diagram of the digital self-learning regulation. 49

3.22 Power supplies with stacked buck converters. 50

3.23 The overall layout of buck converter.

3.24 a) RC Circuit for carrier waveform; b) RC Circuit for modulating waveform. 53

3.25 The main capacitor bank as black box.

3.26 Control waveform of main switch for constant power. 55

3.27 Buck converter input current. $\quad 56$

3.28 Main switch output voltage. $\quad 56$

3.29 Main capacitor bank voltage. $\quad 57$

3.30 Modulator output pulses. $\quad 57$

3.31 SILC Converter schematic. 58

3.32 SILC switching commands and current waveforms. 59

3.33 Dependence of the output current on the phase shift delay. 60

$\begin{array}{ll}3.34 & \text { Phase-shift control characteristic. }\end{array} 60$

3.35 Switches control signals in the range time $0<\mathrm{t} \leq 100 \mathrm{~ms}$. 63

3.36 Switches control signals after $100 \mathrm{~ms}$. 63

3.37 Transformer current primary windings T1' and T1. 65

3.38 Transformers voltage primary windings T1' and T1. 65

3.39 Voltage of the transformers secondary windings T1' and T1 in the 66 configuration with center-tapped transformer.

3.40 Voltage on the rectifier diodes in the configuration with center-tapped transformer. 66

3.41 Voltage of the transformers secondary windings relatively at Graetz bridge 67 configuration.

3.42 Voltage on the rectifier diodes in the configuration with Graetz bridge. 67

$\begin{array}{lll}3.43 & \text { Main capacitor bank voltage. } & 68\end{array}$

3.44 Charging and discharging current of the main capacitor bank. 68 
3.46 Switching loci relatively to hard turn-on and turn-off of a semiconductor device. $\quad 70$

$\begin{array}{ll}3.47 \text { Circuit configuration of turn-off snubber. } & 71\end{array}$

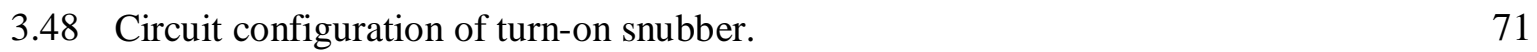

3.49 Switching trajectories with and without turn-on snubber. $\quad 71$

$\begin{array}{lll}3.50 & \text { IGBT circuit symbol and equivalent circuit. } & 72\end{array}$

3.51 Datasheet of CM600HA-12H Power IGBT. 74

$\begin{array}{ll}3.53 & \text { Total power losses depending of snubber capacity. }\end{array}$

$\begin{array}{ll}3.54 \text { IGBT1 turn-off. } & 77\end{array}$

$\begin{array}{ll}3.55 & \text { IGBT2 turn-off. }\end{array}$

$\begin{array}{ll}3.56 & 7 \text { IGBT3 turn-off. }\end{array}$

$\begin{array}{lll}3.57 & \text { IGBT4 turn-off. } & 77\end{array}$

$\begin{array}{lll}3.58 & \text { IGBT1 turn-on. } & 78\end{array}$

$\begin{array}{lll}3.59 & \text { IGBT2 turn-on. } & 78\end{array}$

$\begin{array}{lll}3.60 & \text { IGBT3 turn-on. } & 78\end{array}$

$\begin{array}{lll}3.61 & \text { IGBT4 turn-on. } & 78\end{array}$

4.01 a) The New Muon Lab building; b) the ILC Test Facility at the New Muon Lab 80

4.02 Layout of the beam line and service apparatus: cooling, RF lines, klystron, $\quad 80$ Modulator, etc. in the New Muon Lab complex.

4.03 DC power supply and bouncer modulator design with GTOs switches. 82

4.04 The Fermilab Bouncer Modulator design with IGBTs switches. 82

4.05 A classical 4-stages Marx generator circuit. $\quad 84$

4.06 A 3-stages Marx generator layout; figure a) shows the current charging path, $\quad 85$ while figure b) gives the discharging current path.

4.07 An example of 3-stages Solid State Marx Bank emp88loying ideal charge and 86 discharge IGBTs $\left(\mathrm{S}_{\mathrm{C}}\right.$ and $\left.\mathrm{S}_{\mathrm{D}}\right)$.

4.08 Klystron power supply complex, using our Solid State Marx Modulator $\left(\mathrm{SM}^{2}\right) . \quad 87$

4.09 The $\mathrm{SM}^{2}$ fundamental cell layout. $\quad 88$

4.10 The Main Marx block layout. $\quad 89$ 
4.11 Marx bank efficiency versus the number of Marx stages for various stray stage 90 capacitances. The inset shows a close-up of 10-stages modulator efficiency.

$\begin{array}{ll}4.12 & \text { Simulated single cell output voltage. }\end{array}$

4.13 Simulated single cell output current. $\quad 91$

4.14 Simulated single cell output energy. $\quad 91$

4.15 Simulated Main Marx output voltage; the droop in the output voltage has a value of 93 about $13 \%$.

4.16 Simulated Main Marx output current; the droop in the output current has a value of 93 about $13 \%$.

4.17 Simulated Main Marx output energy. 93

4.18 Overall $\mathrm{SM}^{2}$ Marx Modulator layout. As it is possible to see in the circuit 95 schematic, the Flattener circuit is in series with the Main Marx generator.

4.19 Full SM ${ }^{2}$ Modulator conceptual design, where STS is the Switching Trigger System 96 for the $\mathrm{SM}^{2}$ modulator, $\mathrm{MM}$ is the $\mathrm{SM}^{2}$ Main Marx modulator, the MMDS is the Main Marx Driving System, FDS is the Flattener Driving System and the KEDOSS is the Klystron Energy Droop Out Safety System. PS is the $\mathrm{SM}^{2} \mathrm{DC} / \mathrm{DC}$ switching power supply.

4.20 Sequence of the trigger signals necessary to operate the Flattener charging switches. $\quad 97$

4.21 Pulse width of the trigger signals necessary to operate the Flattener discharging 97 switches.

$\begin{array}{lll}4.22 & \text { Simulated Flattener output voltage. } & 98\end{array}$

4.23 Simulated voltage (a) and current (b) droop as a function of number of cell that 99 failed in the $\mathrm{SM}^{2}$ modulator.

4.24 Simulated $\mathrm{SM}^{2} 120 \mathrm{kV}$ output train pulses; the repetition rate is $10 \mathrm{~Hz} . \quad 100$

4.25 Simulated $\mathrm{SM}^{2}$ output voltage with droop compensation; the voltage droop is 101 about $1 \%$.

4.26 Simulated $\mathrm{SM}^{2}$ output voltage with droop compensation; the voltage droop is 101 about $1 \%$.

4.27 Simulated $\mathrm{SM}^{2}$ output energy.

4.28 Simulated result on the output voltage, in the worst statistical scenario 102

$\begin{array}{ll}\text { A.1. Angular distribution of synchrotron radiation } & 107\end{array}$ 
"Ci sono soltanto due possibili conclusioni: Se il risultato conferma le ipotesi, allora hai appena fatto una misura. Se il risultato è contrario alle ipotesi, allora hai fatto una scoperta."

Enrico Fermi

"If we take everything into account, not only what the ancient knew, but all of what we know today that they didn't know, then I think we must frankly admit that we do not know."

Richard P. Feynman 


\section{Chapter 1}

\section{Physics motivations for the International Linear Collider}

The International Linear Collider (ILC) is the next large project in accelerator based particle physics. It is complementary to the Large Hadron Collider (LHC) in many aspects. Measurements from both machines together will finally shed light onto the known deficiencies of the Standard Model of particle physics and allow to unveil a possible underlying more fundamental theory. Here, the possibilities of the ILC will be discussed with special emphasis on the Higgs sector and on topics with a strong connection to cosmological questions like extra dimensions or dark matter candidates.

\section{$1.1 \quad$ Introduction}

The Standard Model of particle physics (SM) provides a unified and precise description of all known subatomic phenomena. It is consistent at the quantum loop level and it covers distances down to $10^{-18} \mathrm{~m}$ and times from today until $10^{-10} \mathrm{~s}$ after the Big Bang. Despite its success, the SM has some deficiencies which indicate that it is only the effective low energy limit of a more fundamental theory. These deficiencies comprise the absence of experimental evidence for the Higgs particle, its number of free parameters and their values, fine-tuning and stability problems above energies of about $1 \mathrm{TeV}$, and, last but not least, its ignorance of gravity. Furthermore, the SM contains no particle which could account for the cold dark matter observed in the universe. There are good reasons to expect phenomena beyond the SM at the $\mathrm{TeV}$ scale, i.e. in the reach of the immediate generation of new accelerators. Any new physics which solves the hierarchy problem between the electroweak and the Planck scale needs to be close to the former. Experimental hints arise from the fits to electroweak precision data, which require either a Higgs boson mass below $250 \mathrm{GeV} / \mathrm{c}^{2}$ or something else which causes similar loop corrections. Furthermore, most cold dark matter scenarios based on the hypothesis of a weakly interacting massive particle favour masses of about $100 \mathrm{GeV} / \mathrm{c}^{2}$. If there are new particles "around the corner", the LHC is likely to discover them. The ultimate goal, however, is not only to discover new particles, but to measure their properties and interaction with high precision in order to pin down the underlying theory and to determine its parameters. In the unlikely case that the LHC will not find any new particles, the task of the ILC would be to measure the SM parameters with even higher precision than before $[5,6]$ in order to find out what is wrong with today fits that point to a light Higgs and new physics at the $\mathrm{TeV}$ scale. In any case, an electron position collider will provide an invaluable tool complementary to the LHC. 

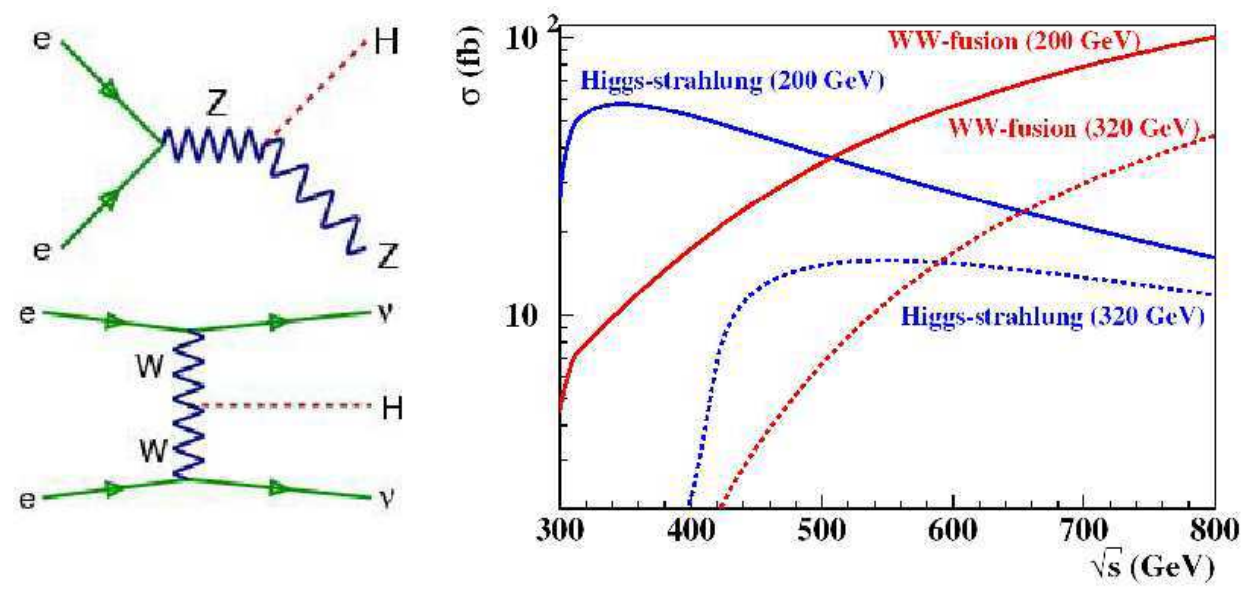

Figure 1.1: Left: Feynman diagrams for Higgsstrahlung (top) and WW fusion (bottom). Right: cross sections of the two processes as function of the center of mass energy for Higgs masses of 200 and $320 \mathrm{GeV} / \mathrm{c}^{2}$ [1].

\subsection{Higgs Boson Physics}

The last unobserved ingredient of the SM is the Higgs boson. It gives mass to the originally mass-less weak gauge bosons and to all quarks and charged leptons, and it is essential to keep the theory finite. The most characteristic property of this fundamental scalar field is that its couplings have to be proportional to the mass of the particles it interacts with - if the interaction with the Higgs field is indeed responsible for the mass generation. If the SM Higgs boson exists, it will be discovered at the LHC once the data from about one year of running time have been analysed. The LHC experiments will also be able to measure quite precisely its mass and the ratios of couplings. The ILC on the other hand would be a factory for Higgs bosons, with as many Higgs events expected per day as the LHC experiments collect per year. The high production rate and the clean environment of an electron positron collider will allow to determine very precisely the complete profile of the Higgs boson and thus to establish the Higgs mechanism experimentally and to prove that this new particle really is the SM Higgs boson. If it turns out that it is not, it might be one of several Higgs bosons appearing in alternative models, e.g. in Supersymmetry (SUSY). Then it is even more important to analyse the profile of all accessible Higgs bosons in order to determine the type and the parameters of the underlying theory. At the ILC, the SM Higgs boson will be produced by two processes Higgsstrahlung and WW fusion, whose Feynman diagrams and cross sections are shown in Figure 1.1. The Higgsstrahlung process, which dominates for center of mass energies up to about $300 \mathrm{GeV} / \mathrm{c}^{2}$ above the Higgs mass, provides the possibility of a decay mode independent mass measurement via the recoil mass against the $\mathrm{Z}^{0}$ boson produced together with the Higgs, which even works for decays to invisible particles. 
With the detectors planned for the ILC, precisions below $0.1 \%$ are achievable over the mass range favoured by the SM. The WW fusion process is a unique tool for determining the total width of the Higgs boson even for low Higgs masses, i.e. $m_{H}<200 \mathrm{GeV} / \mathrm{c}^{2}$, by measuring the total WW fusion cross section and the branching ration $\mathrm{BR}\left(\mathrm{H} \rightarrow \mathrm{WW}^{*}\right)$. At high masses, the Higgs is so broad that its width can be determined directly from its line-shape. The total width then gives access to all couplings via measuring the branching fractions. Figure 1.2 shows the expected precision for the different Higgs branching ratios. Especially disentangling decays to $\bar{b} \bar{b}, c \bar{c}$ and gg is challenging and requires an excellent vertex detector. The only coupling not accessible in decays is the top Higgs Yukawa coupling $\mathrm{g}_{\mathrm{t}}$ due to the high mass of the top quark. At the ILC alone, one would need to collect $1000 \mathrm{fb}^{-1}$ at $800 \mathrm{GeV} / \mathrm{c}^{2}$ to produce enough $\mathrm{e}^{+} \mathrm{e}^{-} \rightarrow t \bar{t} \mathrm{H}$ events to reach a precision of $5 \%$ to $10 \%$ for $\mathrm{g}_{\mathrm{t}}$. A more elegant way to extract $\mathrm{g}_{\mathrm{tt}} \mathrm{H}$ would be to combine the rate measurement of $q \bar{q} / \mathrm{gg} \rightarrow t \bar{t}$ $\mathrm{H}$ (with the Higgs decaying further to $\bar{b} \bar{b}$ or $\mathrm{W}^{+} \mathrm{W}^{-}$) from the $\mathrm{LHC}$, which is proportional to $\mathrm{g}_{\mathrm{t}}{ }^{2} \mathrm{~g}_{\mathrm{b}}{ }^{2} / \mathrm{w}$ with the absolute measurements of $\mathrm{g}_{\mathrm{b}}$ and $\mathrm{g}_{\mathrm{W}}$ from the ILC. The mass of the top quark is one of the most important SM parameters in order to check the overall consistency of the Higgs mechanism. With a scan of the production threshold, the top mass can be measured at the ILC to $50 \div 100 \mathrm{MeV}$ and its width to $3 \div 5 \%$. The ultimate proof of the Higgs mechanism is the measurement of the Higgs self coupling $\lambda$, which allows to map the Higgs potential and check the relation between $\lambda$, the Higgs mass and its vacuum expectation value: $\lambda=\mathrm{m}_{\mathrm{H}}{ }^{2} / 2 \mathrm{v}^{2}$. This needs Higgsstrahlung events, where the Higgs itself radiates off a second Higgs boson, which leads to 6-fermion final states, including 6-jet final states. These require an excellent jet energy resolution, much superior to for example the LEP detectors. Once all Higgs parameters are measured, a global fit to all Higgs properties will answer the question if it really is the SM Higgs boson - or for example a supersymmetric one - even if no other new particle should be observed at the LHC. Even if other particles will have been already discovered, a careful analysis of the Higgs sector is essential to unveil the model beneath the new phenomena and to determine its parameters. Figure 1.2 shows the SM and some MSSM expectations in the $\mathrm{gt}_{\mathrm{t}} \mathrm{g}_{\mathrm{W}}$ plane and the precisions achievable at LHC and at ILC. If Supersymmetry is realised in nature, there will be at least five physical Higgs bosons: two CP-even bosons $\mathrm{h}$ and $\mathrm{H}$, similar to the $\mathrm{SM}$ one, a neutral, $\mathrm{CP}$-odd boson $\mathrm{A}$, and two charged Higgs bosons $\mathrm{H}^{ \pm}$. In contrast to the SM case, the Higgs masses are not free parameters, but depend in on other SUSY and SM parameters. 


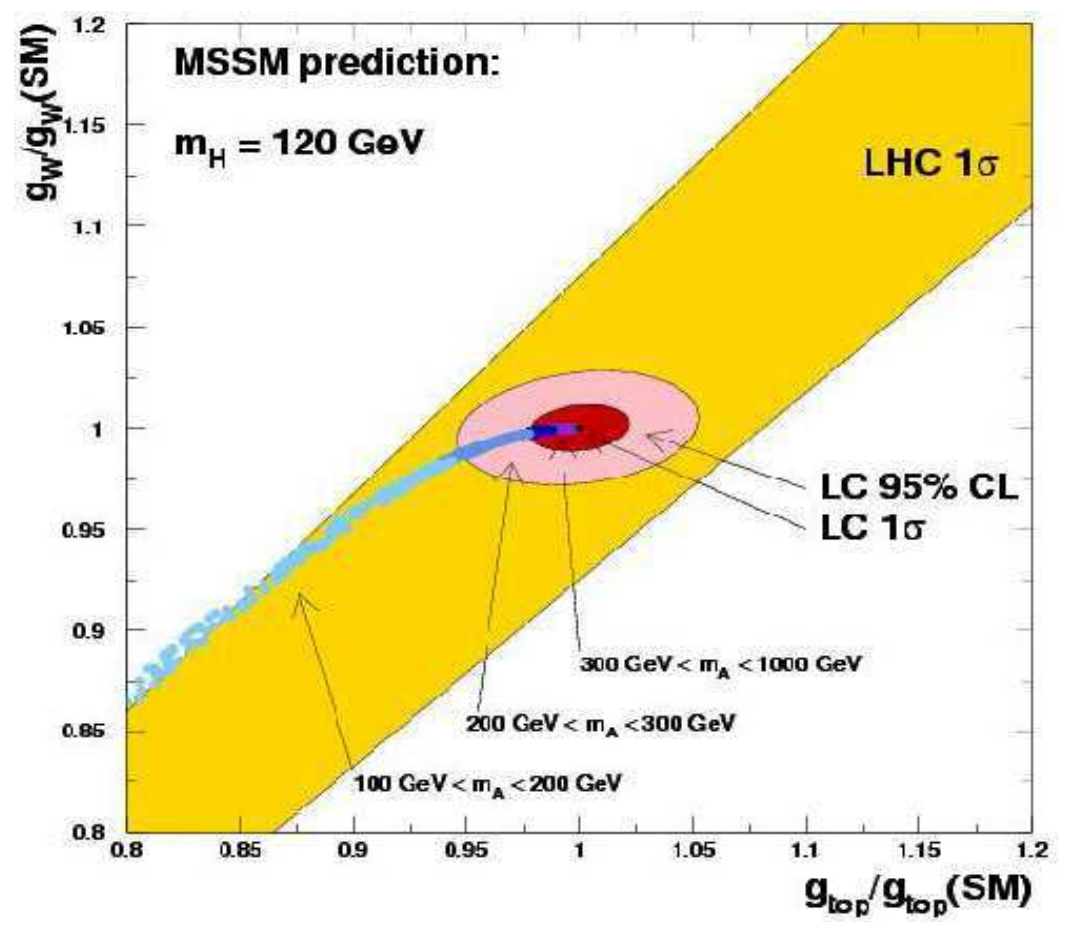

Figure 1.2: $\mathrm{SM}$ and MSSM predictions for $\mathrm{g}_{\mathrm{ttH}}$ versus $\mathrm{g}_{\mathrm{WWH}}$ and expected precision of the corresponding LHC and ILC measurements [1].
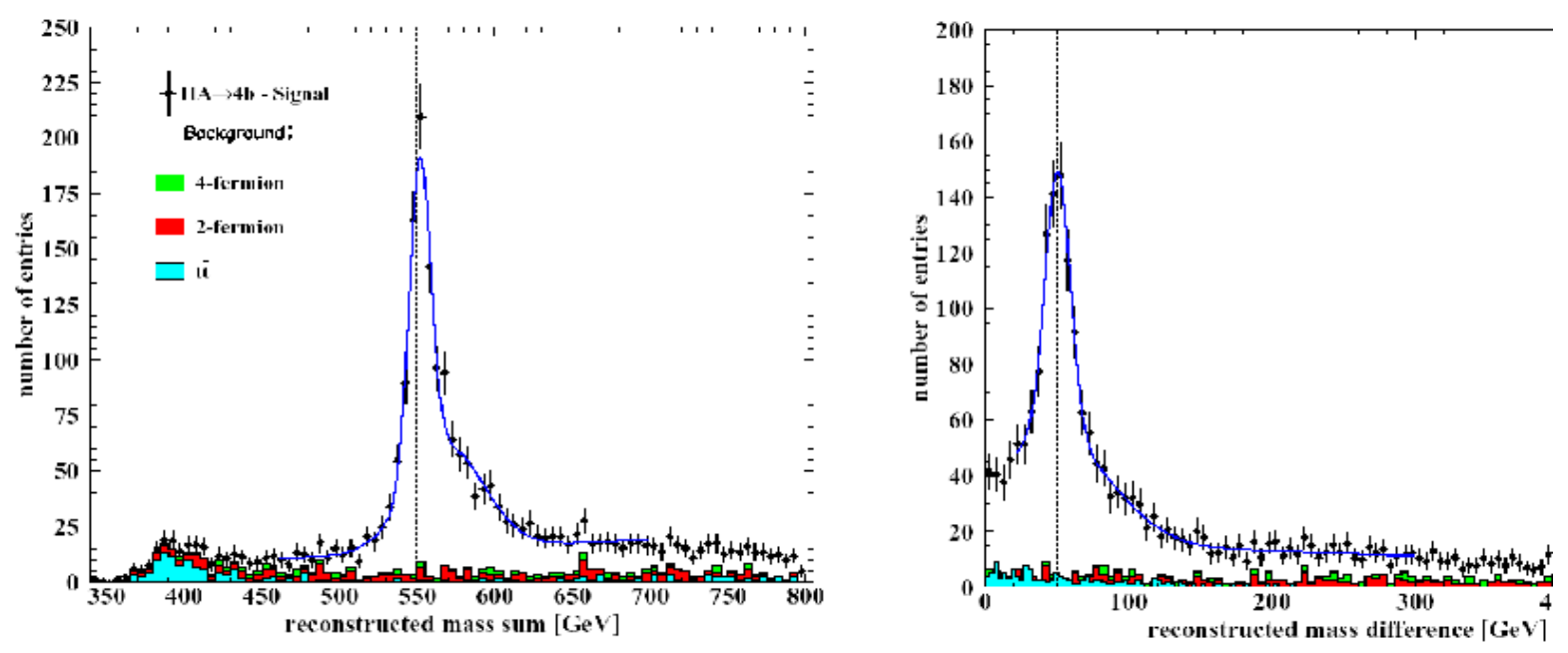

Figure 1.3: Reconstructed mass sum and difference for $\mathrm{HA} \rightarrow 4 \mathrm{~b}$ for masses of $\mathrm{m}_{\mathrm{H}}=250$ $\mathrm{GeV} / \mathrm{c}^{2}$ and $\mathrm{m}_{\mathrm{A}}=300 \mathrm{GeV} / \mathrm{c}^{2}$ at a center of mass energy of $800 \mathrm{GeV} / \mathrm{c}^{2}[1]$. 
At the ILC, associated hA and HA production allows very precise measurements of the SUSY Higgs bosons. As an example, figure 1.3 shows the reconstructed mass sum and difference for $\mathrm{HA} \rightarrow 4 \mathrm{~b}$ for masses of $\mathrm{m}_{\mathrm{H}}=250 \mathrm{GeV} / \mathrm{c}^{2}$ and $\mathrm{m}_{\mathrm{A}}=300 \mathrm{GeV} / \mathrm{c}^{2}$ at a center of mass energy of $800 \mathrm{GeV} / \mathrm{c}^{2}$. The determination of the spin and the $\mathrm{CP}$ quantum numbers of the Higgs bosons via their threshold behaviour, their production angles or even via decays into $\tau$ pairs will then allow disentangling the various Higgs bosons. If there is no light Higgs boson found at the LHC, then the serious questions is: "what makes today precision observables behave as if there were a light Higgs in the loops?" In this case, it is especially important to use the ILC's precision to probe virtual effects. For those, the sensitivity of the ILC reaches far beyond its center of mass energy into the multi-TeV range, in many cases substantially beyond even the LHC reach.

\subsection{Dark Matter \& Extra Dimension}

The notion of additional space-time dimensions appears in many theories beyond the Standard Model of particle physics. Especially models in which only gravity can propagate in the extra dimensions, while all other particles and interactions are confined in the usual four dimensional world, are very attractive because they could explain why gravity appears to be so much weaker than the other interactions. In other words, it provides a solution to the problem of the large hierarchy between the electroweak and the Planck scale. If such a model is realised in nature, then some of the gravitons produced at the ILC via $\mathrm{e}^{+} \mathrm{e}^{-} \rightarrow \gamma \mathrm{G}$ will escape into the extra dimension, producing events with a photon and missing energy and momentum with respect to the initial beam particles. The energy dependence of the cross section for this process is closely correlated to the number of extra dimensions $\delta$. The main background for such processes is SM neutrino production with an additional hard initial state photon, which can be suppressed by one order of magnitude by choosing the right beam polarisations, allowing thus to determine the number of extra dimensions as shown in Figure 1.4. Another intriguing topic for the ILC is the understanding of the dark matter [7] which makes up 23\% of our universe according to recent cosmological observations [2]. The Standard Model of particle physics does not contain a suitable candidate to explain this large amount. One type of candidates which is predicted in many models beyond the SM are Weakly Interacting Massive Particles (WIMPs). In Supersymmetry for example, the Lightest Supersymmetric Particle (LSP) can be such a WIMP candidate, provided it is electrically neutral, not coloured and stable, i.e. if $R$-parity $\left(R_{P}\right)$ is conserved. 

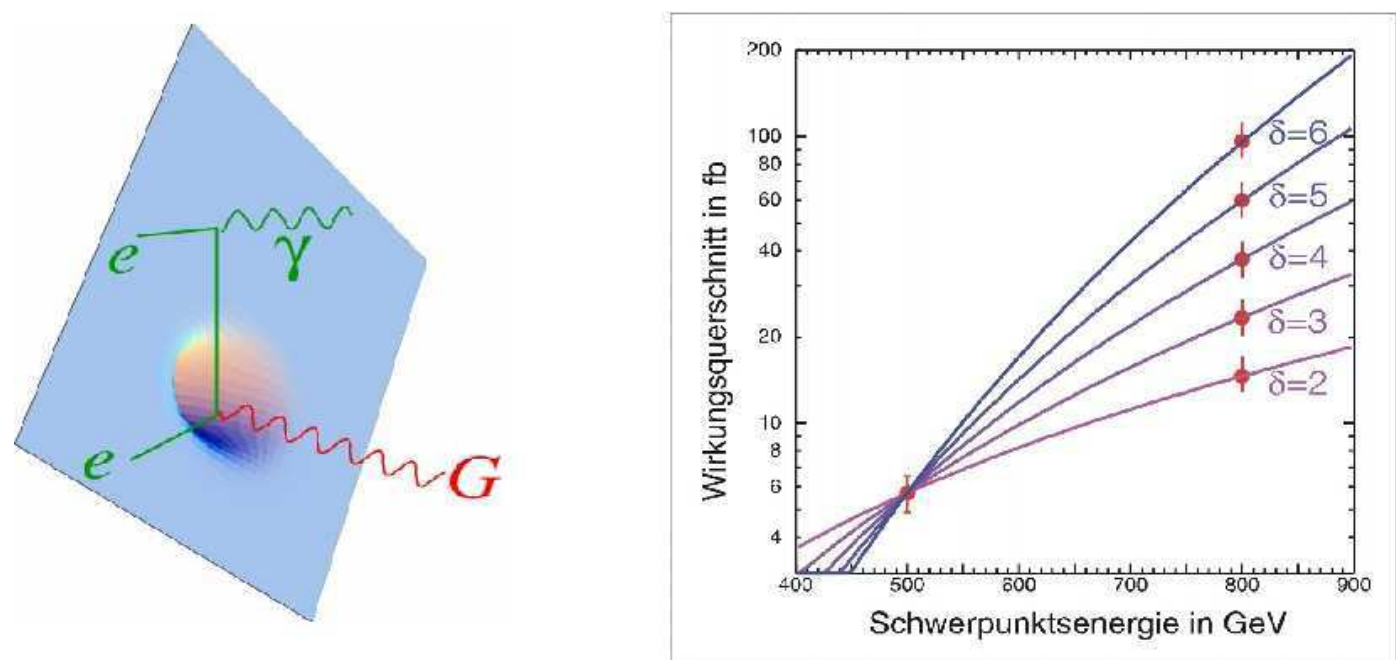

Figure 1.4: Left: Production mechanism for $\mathrm{e}^{+} \mathrm{e}^{-} \rightarrow \gamma \mathrm{G}$, the graviton escapes into extra dimensions. Right: The cross section of this process as a function of the center of mass energy for different numbers of extra dimensions. The points with error bars indicate the precision of the ILC measurements [1].

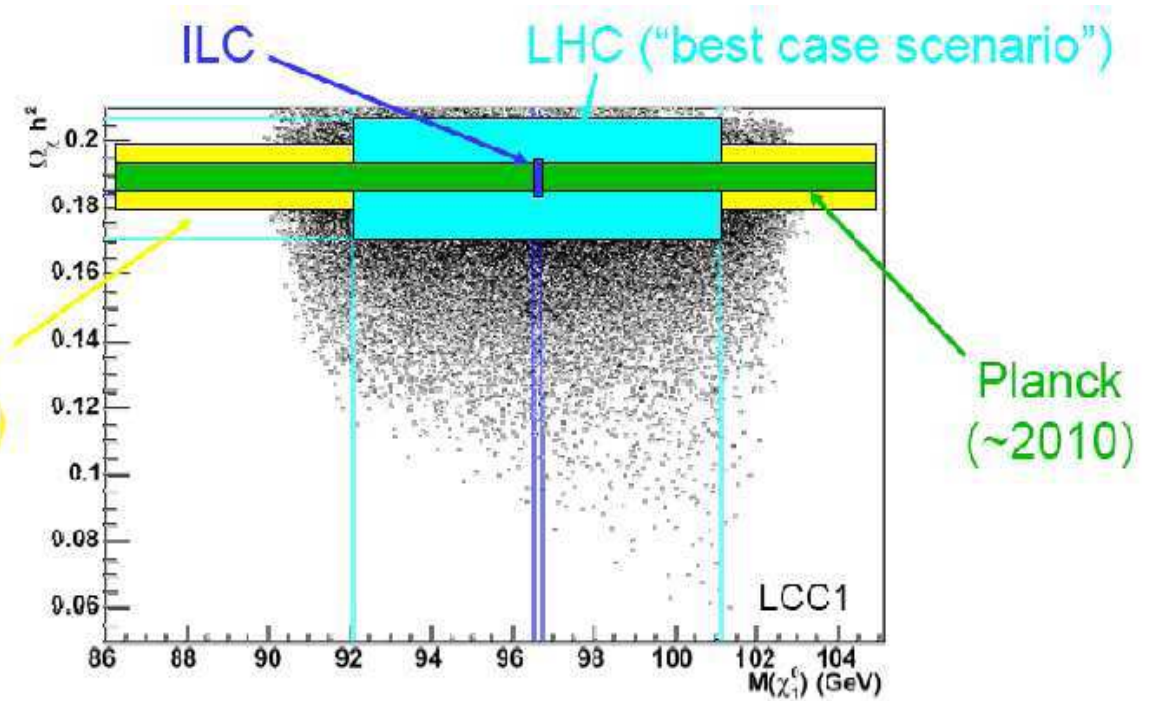

Figure 1.5: Dark matter relic density versus WIMP mass in the LCC1 SUSY scenario. Possible parameter choices are indicated as black dots, which are compared to the sensitivity of present and future measurements from satellite and accelerator based experiments [4]. 
The lightest CP-even Higgs h, for example, must have a mass of less than about $135 \mathrm{GeV} / \mathrm{c}^{2}$. Despite its large center of mass energy, there are regions in the SUSY parameter space where the LHC can see only one of the Higgs bosons, depending on the parameters of the Higgs sector, for example in maximal mixing scenario at intermediate values of $\tan \beta$. In these cases, the additional precision information from the ILC will be especially important. In many SUSY scenarios the lightest neutralino plays this role, which would be discovered at the LHC. The observation and even the mass measurement alone however do not answer the question whether the LSP accounts for the dark matter in the universe. This can only be clarified when all relevant parameters of the model are determined, so that the cross section for all reactions of the WIMPs with themselves and/or other particles and thus the relic density can be calculated. This is illustrated in Figure 1.5, which shows the calculated relic density as a function of the mass of the lightest neutralino in the so called LCC1 scenario. The yellow band shows the current knowledge of the relic density from the WMAP experiment, which is expected to be improved significantly by the Planck satellite in the future (green band). The scattered points result from a scan over the free parameters of this scenario, which can for the same LSP mass yield a large variation in the relic density. The light blue and dark blue boxes indicate the precision of the measurements at the LHC and ILC, respectively. It is evident that only the precision of the ILC can match in an appropriate way the precision of the cosmological observations.

Another unique opportunity for dark matter searches at the ILC opens up independently from specific models. By only assuming that WIMPs annihilate into SM particles, one can use the observed relic density and crossing symmetries to calculate an expected rate for WIMP pair production via $\mathrm{e}^{+} \mathrm{e}^{-} \rightarrow \chi \chi$. The predicted rates [3] show that such events could be observed at the ILC by the detection of an additional hard initial state radiation photon, however further more detailed studies are needed. 


\section{Chapter 2}

\section{The ILC Accelerator Design}

In the past century, physicists have explored smaller and smaller scales, cataloguing and understanding the fundamental components of the universe, trying to explain the origin of mass and probing the theory of extra dimensions. And in recent years, experiments and observations have pointed to evidence that we can only account for a surprising five percent of the universe. The International Linear Collider (ILC) is a proposed linear particle accelerator, consisting of two linear accelerators that face each other (Figure 2.1). ILC will hurl some 10 billion electrons and their anti-particles, positrons, toward each other at nearly the speed of light. Superconducting accelerator cavities operating at temperatures near absolute zero give the particles more and more energy until they smash in a blazing crossfire at the centre of the machine. Stretching approximately 31 kilometers in length, the beams collide 14,000 times every second at extremely high energies $500 \mathrm{GeV}$. Each collision will create an array of new particles that could answer some of the most fundamental questions of all time. The current baseline design allows for an upgrade to a 50-kilometre, 1 trillionelectron-volt $(\mathrm{TeV})$ machine during the second stage of the project. ILC would collide electrons with positrons. The ILC is based on $1.3 \mathrm{GHz}$ superconducting RF cavities operating at a nominal gradient of $35 \mathrm{MeV} / \mathrm{m}$. The collider operates at a repetition rate of $5 \div 10 \mathrm{~Hz}$. The site length is $31 \mathrm{~km}$ for center of mass energy of $500 \mathrm{GeV}$; the site would have to be extended to reach $1 \mathrm{TeV}$. The International Linear Collider's (ILC) most important requirements are:

- an initial center-of-mass (cms) energy up to $500 \mathrm{GeV}$ with the ability to upgrade to 1 $\mathrm{TeV}$;

- an integrated luminosity in the first four years of $500 \mathrm{fb}^{-1}$ at $500 \mathrm{GeV} \mathrm{cms}$ or equivalent at lower energies;

- the ability to scan in energy between 200 and $500 \mathrm{GeV}$ cms.

The installed RF system is capable of accelerating beams for collisions up to center of mass energy of $500 \mathrm{GeV}$. The design peak luminosity of $2 \cdot 10^{34} \mathrm{~cm}^{-2} \mathrm{~s}^{-1}$ at $500 \mathrm{GeV}$ and a collider availability of $75 \%$ should enable to the delivery a total integrated luminosity of about 500

$\mathrm{fb}^{-1}$, in the first four years of physics operations, assuming an annual physics run of 9 months, and a gradual ramp up of luminosity over the four years. 


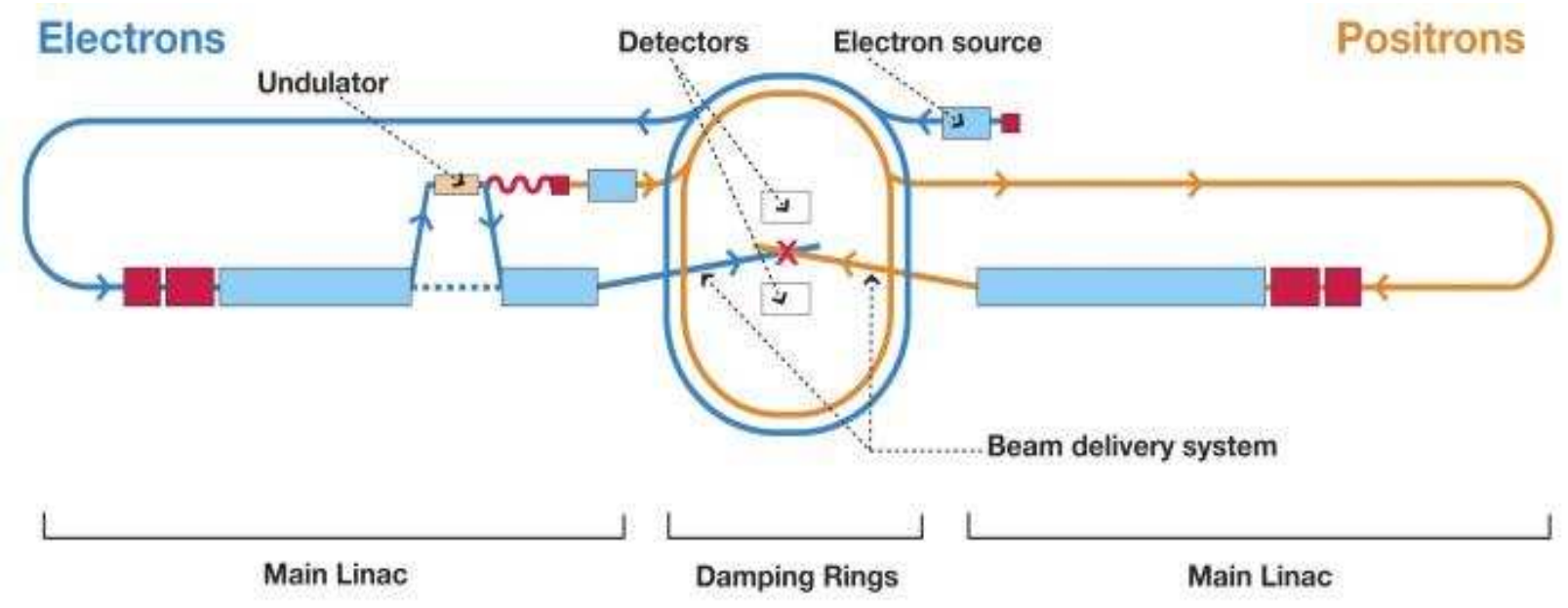

Figure 2.1: A schematic layout of the International Linear Collider.

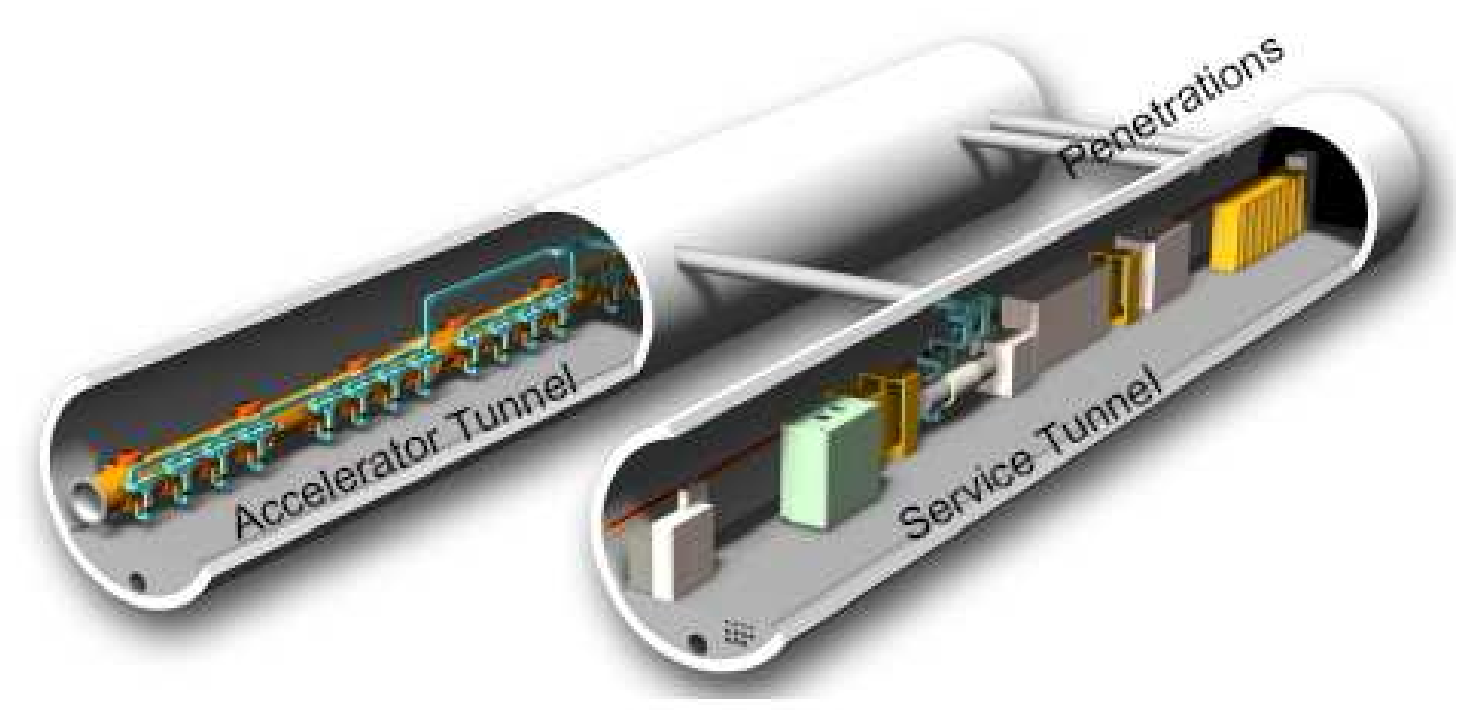

Figure 2.2: Cutaway view of the linac dual-tunnel configuration. On the left you can see the Main Linac tunnel, on the right the service tunnel hosting the klystrons, the klystron modulators and switching power supplies. 


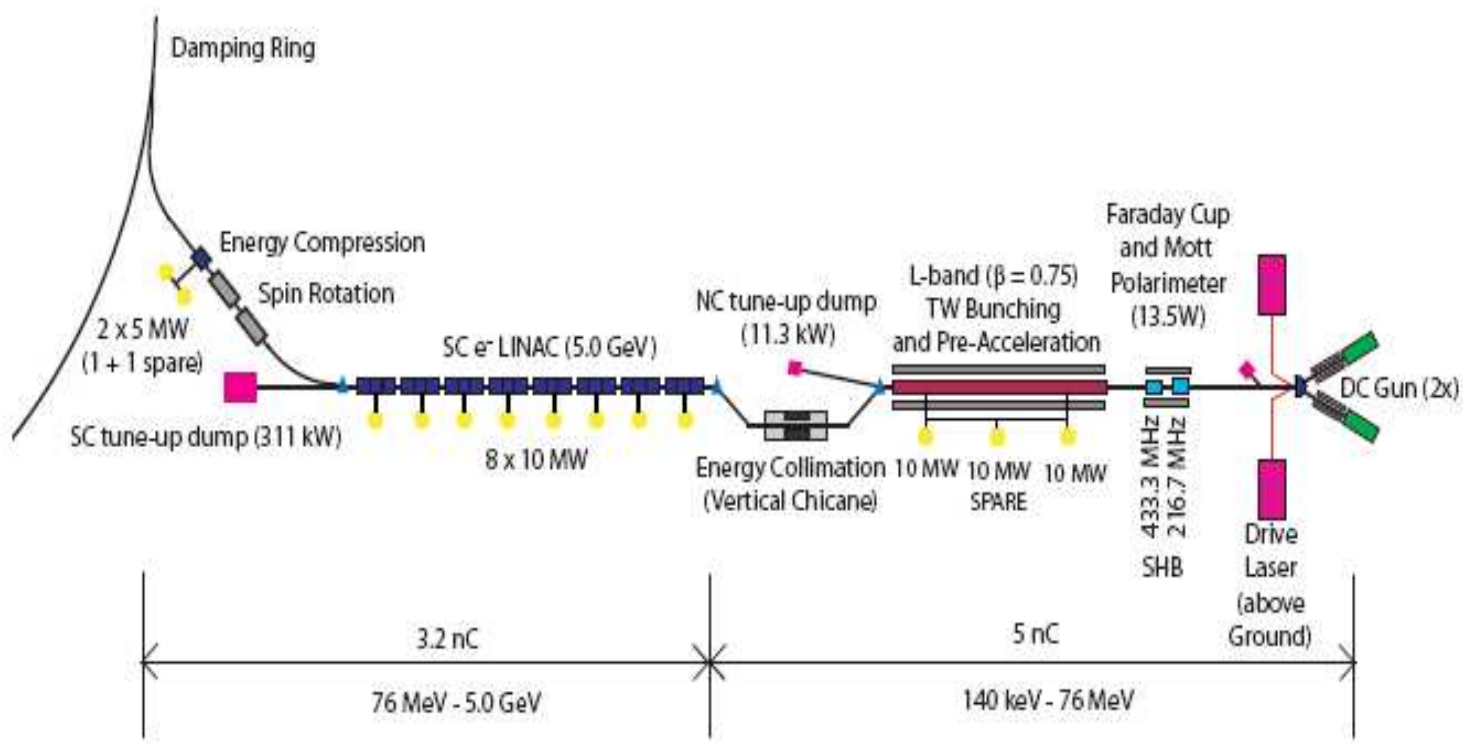

Figura 2.3: Schematic view of the polarized Electron Source.

The energy flexibility has been a consideration throughout the design and essential items to facilitate a future upgrade to $1 \mathrm{TeV}$, such as the length of the beam delivery system and the power rating of the main beam dumps, have been incorporated. The beams are prepared in low energy damping rings that operate at $5 \mathrm{GeV}$ and are $6.7 \mathrm{~km}$ in circumference. They are then accelerated in the main linacs which are around $11 \mathrm{~km}$ per side. Finally, they are focused down to very small spot sizes at the collision point with a beam delivery system that is about $2.2 \mathrm{~km}$ per side. To obtain a peak luminosity of $2 \cdot 10^{34} \mathrm{~cm}^{-2} \mathrm{~s}^{-1}$, the collider requires approximately $230 \mathrm{MW}$ of electrical power.

\subsection{Electron and Positron Sources}

The ILC polarized electron source (Figure 2.3) must produce the required train of polarized electron bunches and transport them to the Damping Ring. The nominal train is 2625 bunches of $2.0 * 10^{10}$ electrons at $5 \mathrm{~Hz}$ with polarization greater than $80 \%$ (Table 2.1). The beam is produced by a laser illuminating a photocathode in a DC gun. Two independent laser and gun systems provide redundancy. Normal-conducting structures are used for bunching and pre-acceleration to $76 \mathrm{MeV}$, after which the beam is accelerated to $5 \mathrm{GeV}$ in a superconducting linac. Before injection into the damping ring, superconducting solenoids rotate the spin vector into the vertical, and a separate superconducting RF structure is used for energy compression. 


\begin{tabular}{|l|c|c|c|}
\hline Parameter & Symbol & Value & Units \\
\hline \hline Electrons per bunch (at gun exit) & $n_{e}$ & $3 \times 10^{10}$ & Number \\
\hline Electrons per bunch (at DR injection) & $n_{e}$ & $2 \times 10^{10}$ & Number \\
\hline Number of bunches & $N_{e}$ & 2625 & Number \\
\hline Bunch repetition rate & $F_{\mu b}$ & 3 & $\mathrm{MHz}$ \\
\hline Bunch train repetition rate & $F_{m b}$ & 5 & $\mathrm{~Hz}$ \\
\hline Bunch length at source & $\Delta t$ & 1 & $\mathrm{~ns}$ \\
\hline Peak current in bunch at source & $I_{a v g}$ & 3.2 & $\mathrm{~A}$ \\
\hline Energy stability & $\mathrm{S}$ & $<5$ & $\% \mathrm{rms}$ \\
\hline Polarization & $P_{e}$ & $80($ min $)$ & $\%$ \\
\hline Photocathode Quantum Efficiency & $\mathrm{QE}$ & 0.5 & $\%$ \\
\hline Drive laser wavelength & $\Lambda$ & $790 \pm 20($ tunable) & $\mathrm{nm}$ \\
\hline Single bunch laser energy & $\mathrm{E}$ & 5 & $\mu J$ \\
\hline
\end{tabular}

Table 2.1: Electrons Source System parameters.

\begin{tabular}{|l|c|c|c|}
\hline Beam Parameters & Symbol & Value & Units \\
\hline \hline Positrons per bunch at IP & $n_{b}$ & $2 \times 10^{10}$ & number \\
\hline Bunches per pulse & $N_{b}$ & 2625 & number \\
\hline Pulse repetition rate & $f_{\text {rep }}$ & 5 & $\mathrm{~Hz}$ \\
\hline Positron energy (DR injection) & $E_{0}$ & 5 & $\mathrm{GeV}$ \\
\hline DR transverse acceptance & $\gamma\left(A_{x}+A_{y}\right)$ & 0.09 & $\mathrm{~m}-\mathrm{rad}$ \\
\hline DR energy acceptance & $\delta$ & \pm 0.5 & $\%$ \\
\hline DR longitudinal acceptance & $A_{l}$ & $\pm 3.4 \times \pm 25$ & $\mathrm{~cm}-\mathrm{MeV}$ \\
\hline Electron drive beam energy & $E_{e}$ & 150 & $\mathrm{GeV}$ \\
\hline Electron beam energy loss in undulator & $\Delta E_{e}$ & 3.01 & $\mathrm{GeV}$ \\
\hline Positron polarization ${ }^{\dagger}$ & $P$ & $\sim 60$ & $\%$ \\
\hline
\end{tabular}

Table 2.2: Nominal Positron Source parameters.

A third polarized electron source $(500 \mathrm{MeV})$ drives the Positron Keep Alive Source (KAS). Polarization is not required in the baseline, but will be required for either the $\mathrm{e}^{-}-\mathrm{e}^{-}$or $\gamma-\gamma$ options. The SLC polarized electron source already meets the requirements for polarization, charge and lifetime. The primary challenge for the ILC source is the long bunch train, which demands a laser system beyond that used at any existing accelerator, and normal conducting structures which can handle high RF power. Both R\&D developments are considered manageable. In other hand, ILC Positron Source uses photo-production to generate positrons (Figure 2.4). 


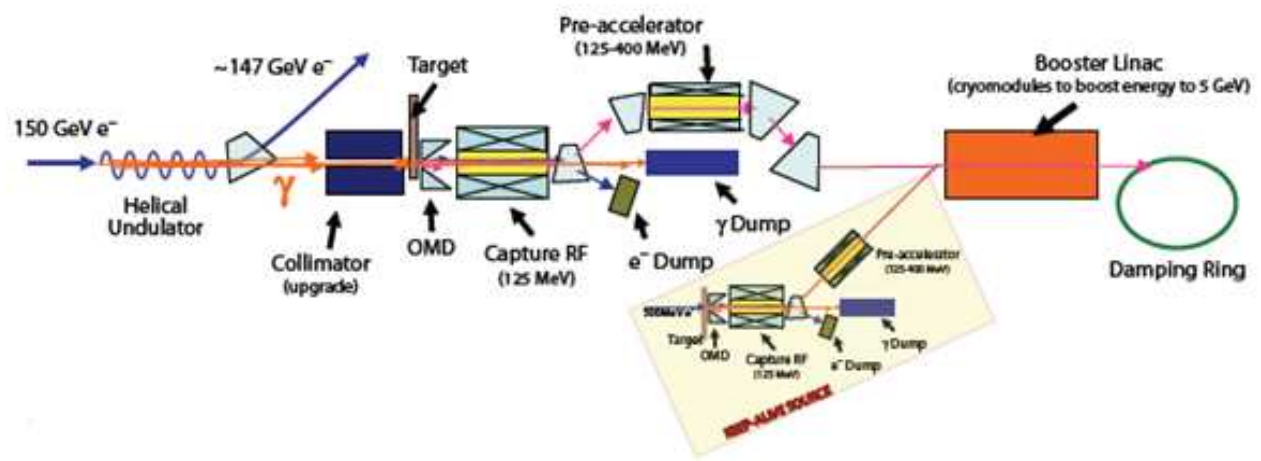

Figura 2.4: Overall layout of the Positron Source.

The electron main linac beam passes through a long helical undulator to generate a multi$\mathrm{MeV}$ photon beam which then strikes a thin metal target to generate positrons in an electromagnetic shower. The positrons are captured, accelerated, separated from the shower constituents and unused photon beam and then are transported to the Damping Ring. Although the baseline design only requires unpolarized positrons, the positron beam produced by the baseline source has a polarization of $\sim 30 \%$, and beam-line space has been reserved for an eventual upgrade to $\sim 60 \%$ polarization (Table 2.2 ). The positron source must perform three critical functions:

- generate a high power multi-MeV photon production drive beam in a suitable short period, high K-value helical undulator;

- produce the needed positron bunches in a metal target that can reliably deal with the beam power and induced radioactivity;

- capture and transport the positron bunch to the ILC Damping Rings with minimal beam loss.

In addition, the Positron Source requires sufficient instrumentation, diagnostics and feedback (feed-forward) systems to ensure optimal operation of the source and ILC.

\subsection{Service Rings}

\subsubsection{Damping Rings}

The ILC damping rings include one electron and one positron ring, each $6.7 \mathrm{~km}$ long, operating at beam energy of $5 \mathrm{GeV}$. The two rings are housed in a single tunnel near the center of the site, with one ring positioned directly above the other. The damping rings must perform three critical functions: 


\begin{tabular}{|l|c|r|}
\hline Parameter & Units & Value \\
\hline \hline Energy & $\mathrm{GeV}$ & 5.0 \\
\hline Circumference & $\mathrm{km}$ & 6.695 \\
\hline Nominal \# of bunches & & 2625 \\
\hline Nominal bunch population & & $2.0 \times 10^{10}$ \\
\hline Maximum \# of bunches & & 5534 \\
\hline Bunch population at max \# of bunches & & $1.0 \times 10^{10}$ \\
\hline Average current & $\mathrm{A}$ & 0.40 \\
\hline Energy loss per turn & $\mathrm{MeV}$ & 8.7 \\
\hline Beam power & $\mathrm{MW}$ & 3.5 \\
\hline Nominal bunch current & $\mathrm{mA}$ & 0.14 \\
\hline RF Frequency & $\mathrm{MHz}$ & 650 \\
\hline Total RF voltage & $\mathrm{MV}$ & 24 \\
\hline RF bucket height & $\%$ & 1.5 \\
\hline Injected betatron amplitude, $A_{x}+A_{y}$ & $\mathrm{~m} . \mathrm{rad}$ & 0.09 \\
\hline Equilibrium $\gamma \epsilon_{x}$ & $\mu \mathrm{m} . \mathrm{rad}$ & 5.0 \\
\hline Chromaticity, $\Xi_{x} / \Xi_{y}$ & & $-63 /-62$ \\
\hline Partition Numbers, $J_{x} / J_{y} / J_{E}$ & & $0.9998 / 1.0000 / 2.0002$ \\
\hline$h$ & $\mathrm{~ms}$ & 14,516 \\
\hline$\nu_{s}$ & & 0.067 \\
\hline$f_{s}$ & $\mathrm{mHz}$ & 3.0 \\
\hline$\alpha_{c}$ & & $5.2 \times 10^{-4}$ \\
\hline$\nu_{x} / \nu_{y}$ & & 25.7 \\
\hline$\sigma_{z}$ & & 12.9 \\
\hline$\sigma_{p} / p$ & & \\
\hline$\tau_{x}$ & & $5.28 \times 10^{-3}$ \\
\hline$\tau_{s}$ & & \\
\hline
\end{tabular}

Table 2.3: Positron Damping Ring parameters; the Electron Damping Ring is identical except for a smaller injected emittance.

- Accept $\mathrm{e}^{-}$and e+ beams with large transverse and longitudinal emittances and produce the low-emittance beams required for luminosity production;

- Damp incoming beam jitter (transverse and longitudinal) and provide highly stable beams for downstream systems;

- Delay bunches from the source to allow feed-forward systems to compensate for pulse- to-pulse variations in parameters such as the bunch charge; 


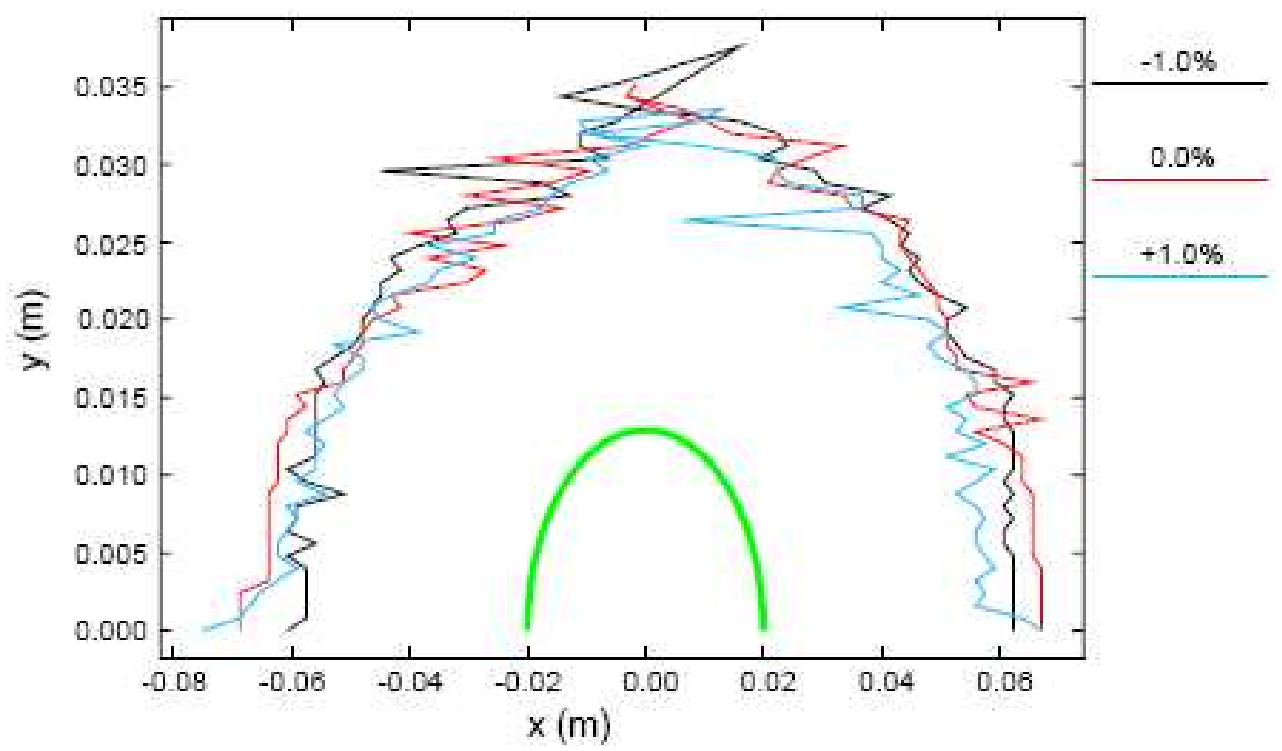

Figure 2.5: Dynamic aperture of ILC Damping Ring (without field or alignment error) for relative momentum errors of $-1 \%, 0 \%$ and $1 \%$ at $\mathrm{x}=44 \mathrm{~m}$ and $\mathrm{y}=18 \mathrm{~m}$. The tick green line represents the size of the injected positron beam.

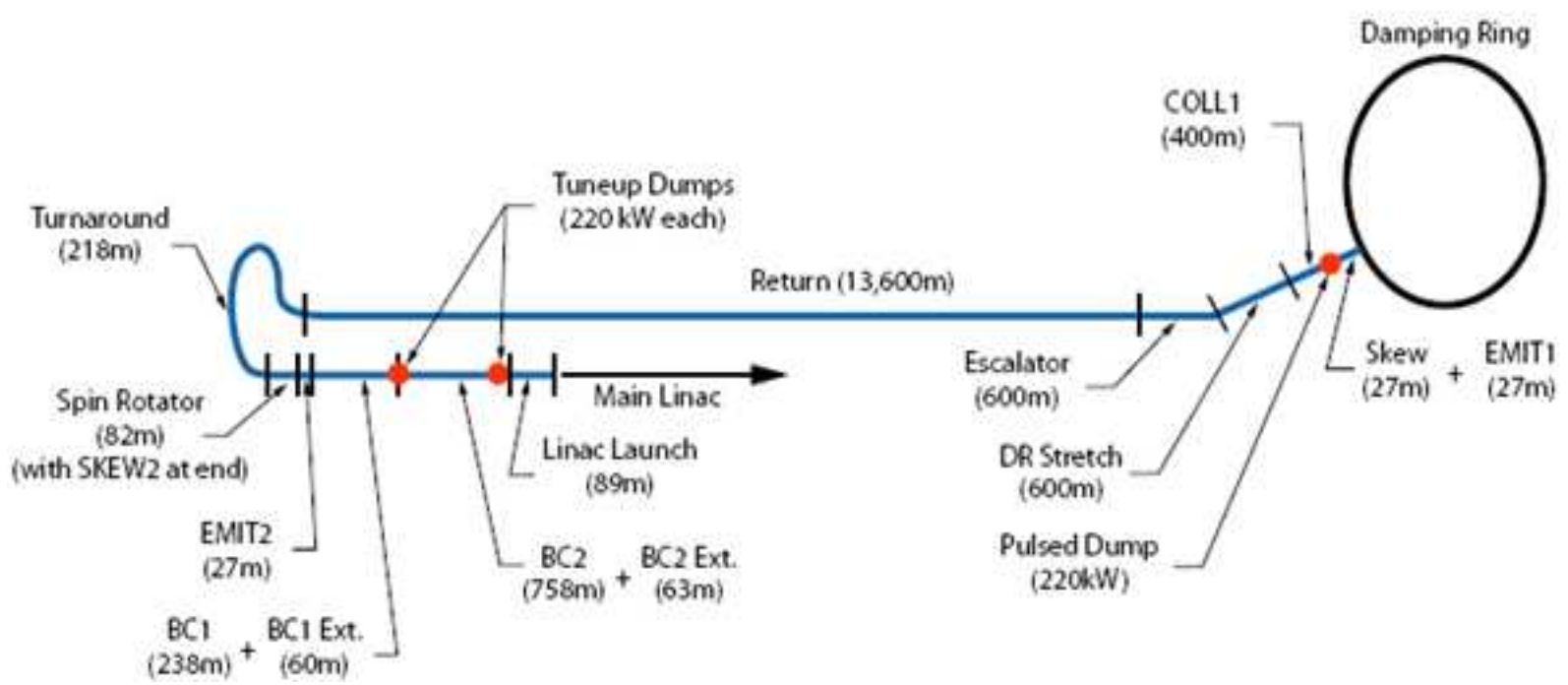

Figure 2.6: Schematic of RTML. 


\begin{tabular}{|c|c|c|}
\hline Parameter & Nominal Value & Low Charge Value \\
\hline Initial energy & \multicolumn{2}{|c|}{$5.0 \mathrm{GeV}$} \\
\hline Initial energy spread & \multicolumn{2}{|c|}{$0.15 \%$} \\
\hline Initial emittances & \multicolumn{2}{|c|}{$8.0 \mu \mathrm{m} \times 20 \mathrm{~nm}$} \\
\hline Initial horizontal beam jitter & \multicolumn{2}{|c|}{$1 \sigma$} \\
\hline Initial bunch length & \multicolumn{2}{|c|}{$9.0 \mathrm{~mm}$} \\
\hline Final bunch length & $0.3 \mathrm{~mm}$ & $0.2 \mathrm{~mm}$ \\
\hline Final energy & $15.0 \mathrm{GeV}$ & $13.7 \mathrm{GeV}$ \\
\hline Final energy spread & $1.5 \%$ & $2.7 \%$ \\
\hline Final horizontal beam jitter & \multicolumn{2}{|c|}{$0.1 \sigma$} \\
\hline ISR emittance growth & $0.9 \mu \mathrm{m}$ & $0.8 \mu \mathrm{m}$ \\
\hline Emittance budget & \multicolumn{2}{|c|}{$1 \mu \mathrm{m} \times 4 \mathrm{~nm}$} \\
\hline
\end{tabular}

Table 2.4: Basic beam parameters of the RTML.

The damping ring system includes the injection and extraction systems, which includes sections of transport lines matching to the sources (upstream of the damping rings) and the RTML system (downstream of the damping rings).

\subsubsection{Ring to Main Linac}

The ILC Ring to Main Linac (RTML) is responsible for transporting and matching the beam from the Damping Ring to the entrance of the Main Linac.

The RTML must perform several critical functions:

- transport of the electron and positron beams from the damping rings, at the center of the ILC accelerator complex, to the upstream ends of their respective linacs ("geometry matching");

- collimation of the beam halo generated in the damping ring;

- rotation of the spin polarization vector from the vertical to any arbitrary angle required at the IP;

- compression of the long Damping Ring bunch length by a factor of $30 \sim 45$ to provide the short bunches required by the Main Linac and the IP; 


\begin{tabular}{|l|r|l||l|r|l|}
\hline Parameter & Value & Units & Parameter & Value & Units \\
\hline \hline Initial beam energy & 15 & $\mathrm{GeV}$ & Initial $\gamma \epsilon_{x}$ & 8.4 & $\mu \mathrm{m}$ \\
\hline Final beam energy & 250 & $\mathrm{GeV}$ & Final $\gamma \epsilon_{x}$ & 9.4 & $\mu \mathrm{m}$ \\
\hline Particles per Bunch & $2 \times 10^{10}$ & & Initial $\gamma \epsilon_{y}$ & 24 & $\mathrm{~nm}$ \\
\hline Beam current & 9.0 & $\mathrm{~mA}$ & Final $\gamma \epsilon_{y}$ & 34 & $\mathrm{~nm}$ \\
\hline Bunch spacing & 369 & $\mathrm{~ns}$ & $\sigma_{z}$ & 0.3 & $\mathrm{~mm}$ \\
\hline Bunch train length & 969 & $\mu \mathrm{s}$ & Initial $\sigma_{E} / E$ & 1.5 & $\%$ \\
\hline Number of bunches & & 2625 & Final $\sigma_{E} / E\left(\mathrm{e}^{-}, \mathrm{e}^{+}\right)$ & $0.14,0.10$ & $\%$ \\
\hline Pulse repetition rate & 5 & $\mathrm{~Hz}$ & Beam phase wrt RF crest & 5 & $\circ$ \\
\hline
\end{tabular}

Table 2.5: Nominal beam parameters in the ILC Main Linac.

\subsection{Main Linacs}

The two main linacs accelerate the electron and positron beams from their injected energy of $15 \mathrm{GeV}$ to the final beam energy of $250 \mathrm{GeV}$ over a combined length of $23 \mathrm{~km}$. This must be accomplished while preserving the small bunch emittances, which requires precise orbit control based on data from high resolution beam position monitors. The linacs utilize L-band $(1.3 \mathrm{GHz})$ superconducting technology, with nine-cell standing-wave niobium cavities operating at an average gradient of $31.5 \mathrm{MV} / \mathrm{m}$ in a $2 \mathrm{~K}$ super-fluid helium bath. The choice of operating frequency is a balance between the high cavity cost due to size at lower frequency and the lower sustainable gradient due to increased surface resistivity at higher frequency. The accelerator gradient is somewhat higher than that typically achievable today and assumes that further progress will be made during the next few years in the aggressive program that is being pursued to improve cavity performance. The Table 2.5 lists the key beam parameters in the main linac. The rms bunch length remains constant along the linacs, while the bunch fractional energy spread decreases roughly as $\mathrm{E}_{0} / \mathrm{E}$, where $\mathrm{E}$ is the beam energy and $E_{0}$ is the initial main linac beam energy. Bunches are phased $5^{\circ}$ off-crest to minimize their energy spread. No BNS energy spread is included to suppress resonant headto-tail bunch trajectory growth as the short-range wake-field is fairly weak. For this same reason, the focusing strength of the quadrupole lattice in the linacs is kept fairly weak to reduce emittance growth from quadrupole misalignments. 


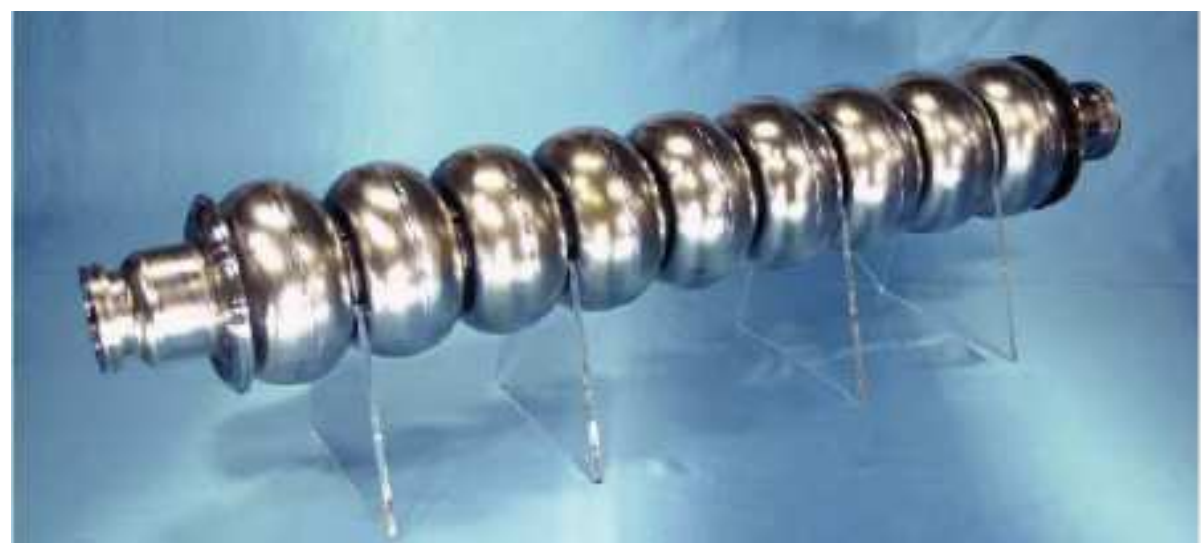

Figure 2.7: 9-cells Superconducting RF Cavity.

\subsection{The ILC Accelerating System}

\subsubsection{Superconducting cavities}

The accelerating gradient in the ILC main linac is supplied by over 16,000 9-cell superconducting RF cavities (Figure 2.7), grouped into approximately $12.6 \mathrm{~m}$ long cryomodules. Another 1200 9-cell cavities provide acceleration in the sources and bunch compressors. The baseline cavities use the TESLA design developed at DESY over the past 10 years. The cavities are qualified at $35 \mathrm{MV} / \mathrm{m}$ gradient in a vertical test and operated at an average gradient of $31.5 \mathrm{MV} / \mathrm{m}$. At these gradients, piezoelectric tuners are required to compensate for Lorentz force detuning. The TESLA 9-cell superconducting cavity was chosen as the baseline design because it has achieved the highest qualification gradients to date for multi-cell cavities, approximately within the range required for ILC. There is significant operational experience with these cavities and it has been demonstrated with beam that accelerating gradients of greater then $30 \mathrm{MV} / \mathrm{m}$ are possible after full installation in a cryomodule. Each 9 cell cavity consists of nine accelerating cells between two end group sections. One end group has a port for coupling RF power from the power source into the structure, and the other end has a port for a field sampling probe used to determine and control the accelerating gradient. Each of these ports accepts an electric field antenna required for qualification and operation. Each end group also has a resonant higher order mode (HOM) coupler structure with a probe port and small electric field antenna for extracting HOM power and for diagnostics. In the process of building a cryomodule, these cavity structures are cleaned, tested and placed in a helium jacket for cooling together with additional peripheral components assembled on them dressing the cavity. 


\begin{tabular}{|l|c|}
\hline Parameter & Value \\
\hline \hline Type of accelerating structure & Standing Wave \\
\hline Accelerating Mode & TM $_{010}, \pi$ mode \\
\hline Fundamental Frequency & $1.300 \mathrm{GHz}$ \\
\hline Average installed gradient & $31.5 \mathrm{MV} / \mathrm{m}$ \\
\hline Qualification gradient & $35.0 \mathrm{MV} / \mathrm{m}$ \\
\hline Installed quality factor & $\geq 1 \times 10^{10}$ \\
\hline Quality factor during qualification & $\geq 0.8 \times 10^{10}$ \\
\hline Active length & $1.038 \mathrm{~m}$ \\
\hline Number of cells & 9 \\
\hline Cell to cell coupling & $1.87 \%$ \\
\hline Iris diameter & $70 \mathrm{~mm}$ \\
\hline $\mathrm{R} / \mathrm{Q}$ & $1036 \Omega$ \\
\hline Geometry factor & $270 \Omega$ \\
\hline $\mathrm{E}_{\text {peak }} / \mathrm{E}_{\text {acc }}$ & 2.0 \\
\hline $\mathrm{B}_{\text {peak }} / \mathrm{E}_{\text {acc }}$ & $4.26 \mathrm{mT} \mathrm{MV}$ \\
\hline Tuning range & $\pm 300 \mathrm{kHz}$ \\
\hline$\Delta \mathrm{f} / \Delta \mathrm{L}$ & $315 \mathrm{kHz} / \mathrm{mm}^{-1}$ \\
\hline Number of HOM couplers & 2 \\
\hline
\end{tabular}

Table 2.6: ILC 9-cell Superconducting Cavity layout parameters.

\begin{tabular}{|l|c||l|c|}
\hline Element & $\begin{array}{c}\text { Impurity content } \\
\text { in ppm (wt) }\end{array}$ & Property & Value \\
\hline \hline $\mathrm{Ta}$ & $\leq 500$ & RRR & $\geq 300$ \\
\hline $\mathrm{W}$ & $\leq 70$ & Grain size & $\approx 50 \mu \mathrm{m}$ \\
\hline $\mathrm{Ti}$ & $\leq 50$ & Yield strength & $>50 \mathrm{MPa}$ \\
\hline $\mathrm{Fe}$ & $\leq 30$ & Tensile strength & $>100 \mathrm{MPa}$ \\
\hline $\mathrm{Mo}$ & $\leq 50$ & Elongation at break & $30 \%$ \\
\hline $\mathrm{Ni}$ & $\leq 30$ & Vickers hardness & \\
\hline $\mathrm{H}$ & $\leq 2$ & HV 10 & $\leq 50$ \\
\hline $\mathrm{N}$ & $\leq 10$ & & \\
\hline $\mathrm{O}$ & $\leq 10$ & & \\
\hline $\mathrm{C}$ & $\leq 10$ & & \\
\hline
\end{tabular}

Table 2.7: Properties of high-RRR (residual resistivity ratio) Niobium suitable for use in ILC Cavities.

A dressed cavity contains one 9-cell niobium cavity structure, coarse and fine tuners for adjusting the frequency of the structure, magnetic shielding material to minimize the cavity losses, a variable coupling high power input antenna for powering the cavity, an electric field sampling antenna and two higher order mode electric field antennas. Nine of these dressed cavities are usually connected into a string and are a subcomponent of a superconducting cryomodule. The basic design parameters for this cavity are listed in Table 2.6 and 2.7. 


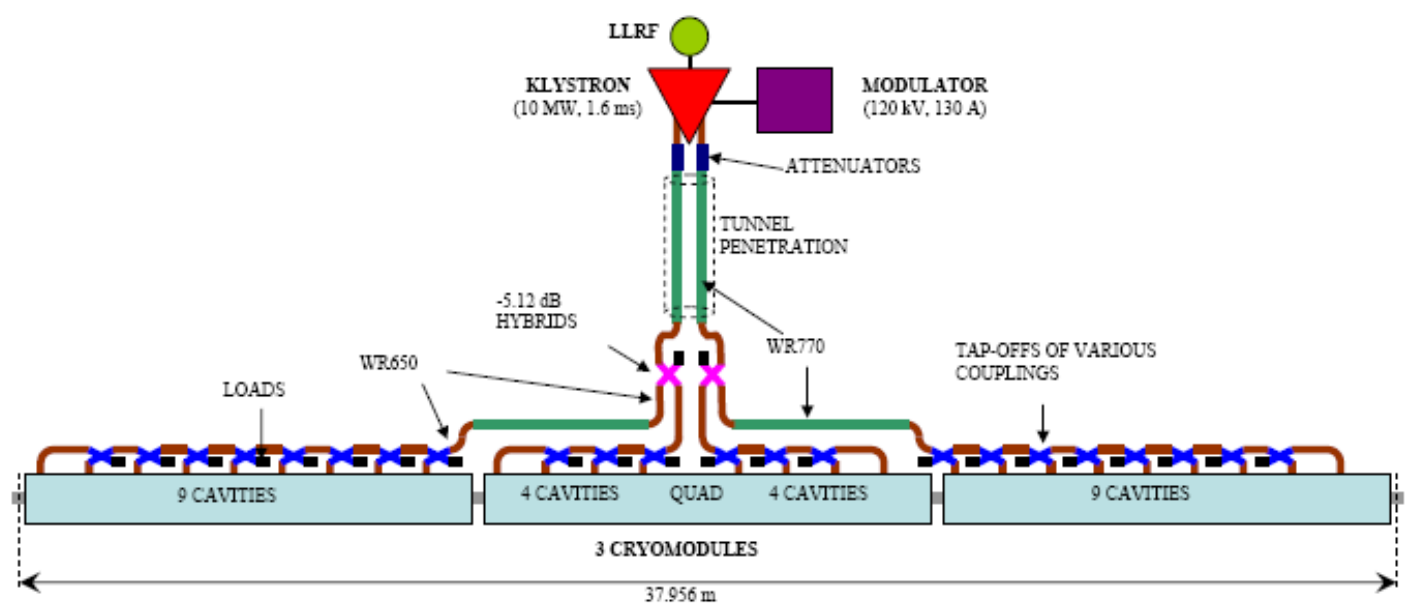

Figure 2.8: RF unit diagram showing the basic waveguide distribution layout between the klystron and 26 cavities in three cryomodules.

\subsubsection{RF distribution system}

The main linacs are composed of RF units whose layout is illustrated in Figure 2.8 and whose parameters are listed in Table 2.8. Each unit has a stand-alone RF source that powers three contiguous cryomodules containing a total of 26 cavities (with 9,8 and 9 cavities in each cryomodule, respectively). The RF source includes a high-voltage modulator, a $10 \mathrm{MW}$ klystron and a waveguide system that distributes the RF power to the cavities. It also includes the low-level RF (LLRF) system to regulate the cavity field levels, interlock systems to protect the source components, and the power supplies and support electronics associated with the operation of the source. To facilitate maintenance and limit radiation exposure, the RF source is housed mainly in a separate service tunnel that runs parallel to the beam tunnel. The modulator is a conventional pulse-transformer type with a bouncer circuit to compensate the voltage droop that occurs in the main storage capacitor during the pulse. The modulator produces $120 \mathrm{kV}, 130 \mathrm{~A}, 1.6 \mathrm{~ms}, 5-10 \mathrm{~Hz}$ pulses with an efficiency of $83 \%$, including the charging supply and rise time losses. These high voltage pulses power a multi-beam klystron (MBK) that amplifies $100 \mathrm{~W}, 1.6 \mathrm{~ms}$ RF pulses from the LLRF system up to $10 \mathrm{MW}$. This klystron has higher power and improved efficiency (65\% goal) relative to commercial $5 \mathrm{MW}$ tubes $(40-45 \%)$. The dual outputs of the klystron feed into two waveguides, each carrying half the power, which runs roughly $11 \mathrm{~m}$ to the linac through a penetration between the service tunnel and the main tunnel. High-power in-line attenuators allow more power to be sent through one arm than the other to accommodate different average gradient capabilities in the sets of cavities they feed. The penetration emerges approximately at the center of the middle cryomodule of the unit. Here, a hybrid splitter divides the power in each waveguide with a $4: 9$ ratio $(-5.12 \mathrm{~dB})$. 


\begin{tabular}{|l|r|l|}
\hline Parameter & Value & Units \\
\hline \hline Modulator overall efficiency & 82.8 & $\%$ \\
\hline Maximum klyston output power & 10 & $\mathrm{MW}$ \\
\hline Klystron efficiency & 65 & $\%$ \\
\hline RF distribution system power loss & 7 & $\%$ \\
\hline Number of cavities & 26 & \\
\hline Effective cavity length & 1.038 & $\mathrm{~m}$ \\
\hline Nominal gradient with $22 \%$ tuning overhead & 31.5 & $\mathrm{MV} / \mathrm{m}$ \\
\hline Power limited gradient with $16 \%$ tuning overhead & 33.0 & $\mathrm{MV} / \mathrm{m}$ \\
\hline RF pulse power per cavity & 293.7 & $\mathrm{~kW}$ \\
\hline RF pulse length & 1.565 & $\mathrm{~ms}$ \\
\hline Average RF power to 26 cavities & 59.8 & $\mathrm{~kW}$ \\
\hline Average power transferred to beam & 36.9 & $\mathrm{~kW}$ \\
\hline
\end{tabular}

Table 2.8: RF Unit parameters.

The lower power output of each splitter feeds half the center cryomodule and the higher power output is carried approximately $6 \mathrm{~m}$ to one of the outer cryomodules (see Figure 2.8). Along each cryomodule, RF power is equally distributed among the cavities in a linear waveguide system, passing through a series of hybrid-style 4-port tap-offs. These tap-offs couple the appropriate sequential fraction $(1 / 8,1 / 7, \ldots, 1 / 2$ or $1 / 4,1 / 3,1 / 2)$ of the power remaining in the line to all but the last cavity, which is directly fed the remainder. The nominal power required in each cavity is $293.7 \mathrm{~kW}$. Between the tap-offs, the remainder of the 1.326 m coupler spacing is filled with modified straight waveguide sections whose width is symmetrically tapered, with 1/4-wave transformer matching steps, varying the guide wavelength to roughly yield the proper inter-cavity phasing. Between each tap-off output and its associated cavity coupler are a circulator, a three-stub tuner, and a diagnostic directional coupler (see Figure 2.9). The three-stub tuner allows fine adjustment of both cavity phase and external coupling. The circulator, with a load on its third port (thus technically an isolator), absorbs RF power reflected from the standing-wave cavity during filling and emitted during discharge. It provides protection to the klystron and isolation between cavities. A couple of E-plane waveguide U-bends are also needed to keep the system compact and feed into the downward pointed coupler flange, and a short semi-flexible section is included to relieve stress and ease alignment tolerances. 


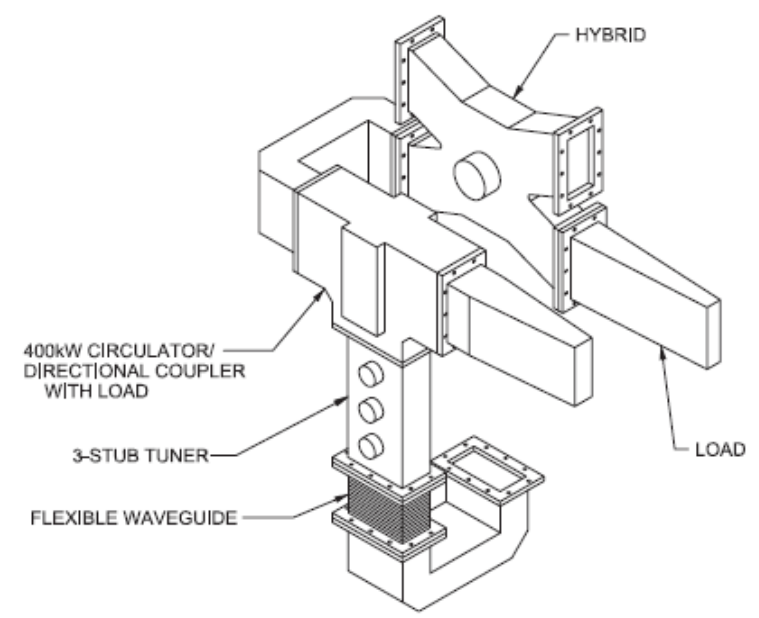

Figure 2.9: Waveguide circuit from tap-off hybrid to coupler input, showing the various components (except for the directional coupler).

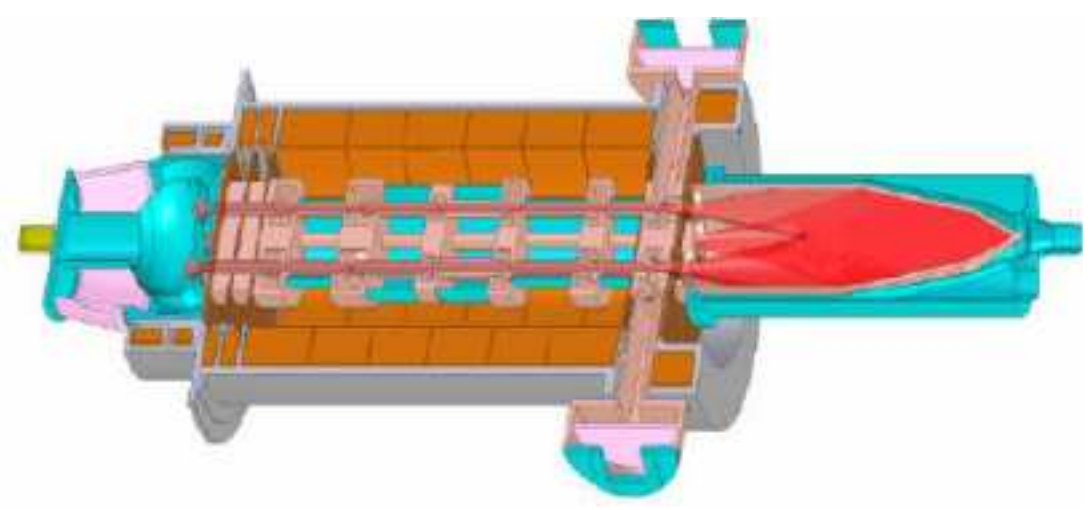

Figure 2.10: Toshiba 3736 Multi-Beam Klystron.

\subsubsection{Klystron}

The accelerating gradient for the ILC main linacs is supplied by superconducting $1.3 \mathrm{GHz}$ cavities powered by $56010 \mathrm{MW}$ RF stations, each with a modulator, klystron and RF distribution system. Another 86 identical klystron/modulator systems are used in the e+ and $\mathrm{e}^{-}$Sources and RTML bunch compressors. The damping ring RF power is supplied by 650 $\mathrm{MHz}$ superconducting cavities powered by 1.2 MW CW klystrons. These are fed from a DC charging supply and do not have modulators. There are also a few special purpose S-band RF stations for instrumentation and a $3.9 \mathrm{GHz}$ RF station to power the crab cavities near the Interaction Point. 
The ILC Accelerator Design

\begin{tabular}{|l|l|}
\hline Parameter & Specification \\
\hline \hline Frequency & $1.3 \mathrm{GHz}$ \\
\hline Peak Power Output & $10 \mathrm{MW}$ \\
\hline RF Pulse Width & $1.565 \mathrm{~ms}$ \\
\hline Repetition Rate & $5 \mathrm{~Hz}$ \\
\hline Average Power Output & $78 \mathrm{~kW}$ \\
\hline Efficiency & $65 \%$ \\
\hline Saturated Gain & $\geq 47 \mathrm{db}$ \\
\hline Instantaneous 1 db BW & $>3 \mathrm{MHz}$ \\
\hline Cathode Voltage & $\leq 120 \mathrm{kV}$ \\
\hline Cathode Current & $\leq 140 \mathrm{~A}$ \\
\hline Power Asymmetry & $\leq 1 \%$ \\
\hline Lifetime & $>40,000$ hours \\
\hline
\end{tabular}

Table 2.9: 10MW MBK parameters.

The 10 MW L-band source in the ILC baseline design is a Multi-Beam Toshiba Klystron (MBK) $[8,9,10,11]$, chosen as a result of ten years of R\&D for TESLA and the European XFEL. The MBK is a design approach for linear beam tubes that achieves higher efficiency by using multiple low space charge (low perveance) beams. This allows MBKs to operate at a lower voltage yet with a higher efficiency than simpler single round beam klystrons, and provides a cost-effective and simplified design configuration for the ILC RF systems. MBK prototypes have been successfully built for the XFEL by three major electron tube manufacturers: Thales, CPI and Toshiba (Figure 2.11). These prototypes were designed for essentially the same peak RF output power specifications as required at ILC, yet with nearly twice the duty cycle as required for the XFEL. All of these manufacturers have extensive past experience in bringing prototype klystrons of state- of-the-art designs into production models, and they are regarded as fully capable of ramping up and producing the required quantities of MBKs to meet the delivery schedule for the construction of the ILC. A summary of the MBK specifications is shown in Table 2.9. The RF design of the MBK klystron has matured through several iterations of design and testing, and today essentially all aspects of the electrical design are considered solved, in particular, the choice of resonant frequencies to use for the cavities within the klystron body, the beam focusing and others. Test results for all three manufacturers are summarized in Figure 2.12. The three most important technical issues for the MBK are lifetime, manufacturability, and reliability. Lifetime for linear beam tubes is dominated by cathode performance. Both the CPI and Toshiba MBKs have gun designs with cathode loading close to $2 \mathrm{~A} / \mathrm{cm}^{2}$. 


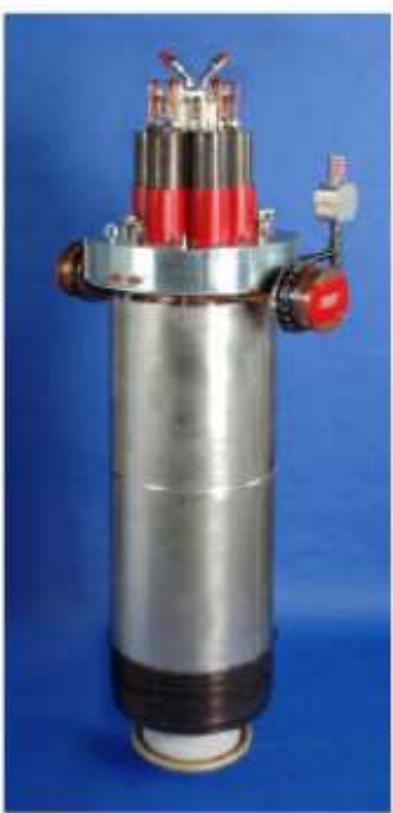

a)

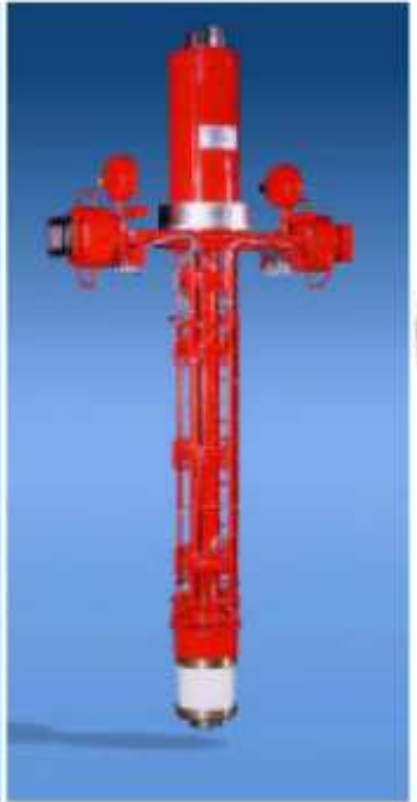

b)

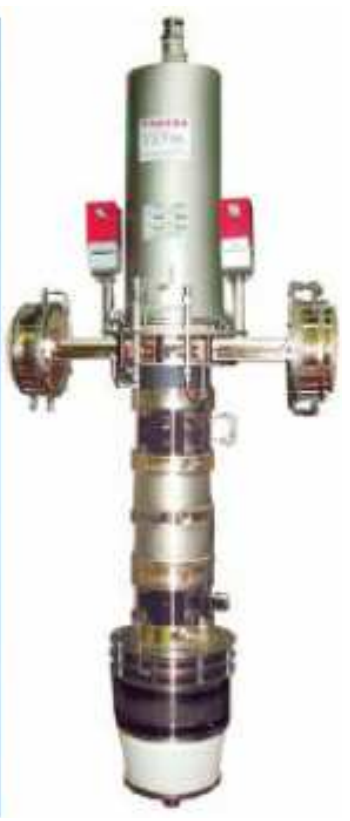

c)

Figure 2.11: (a) CPI VKL-8301 (b) Thales TH 1801 (c) Toshiba MBK E3736.

For an M-type dispenser cathode, this low current density corresponds to a lifetime in excess of 50,000 hours. However, this lifetime has to be confirmed by suitable long-term operation tests. The "lifetime" quoted in Table 2.9 is the time during which the klystron can operate at the design performance specifications.

Construction of the MBK is inherently more complex than that of single-beam klystrons due to the several linear beam tubes being built into a single vacuum envelope. The number of braze joints, the fixturing and tooling, and the processes required to successfully construct, bakeout, and test an MBK are issues that require attention in developing an efficient assembly procedure that reduces the unit cost. For reliable performance, a robust thermal design of the output circuit (output cavity, waveguide, and RF window) is important. Since ILC MBK klystrons are being built for the European XFEL, where they will operate at nearly twice the duty cycle of the ILC, there will be significant thermal/mechanical margin when operated for ILC specifications. The XFEL, however, does not require operation at full power, so reliability at $10 \mathrm{MW}$ must also be demonstrated. A remaining open issue is that the existing prototypes are vertical klystrons but a horizontal version is required for installation in the tunnel. While this is an engineering challenge, DESY is already working with the manufacturers to produce a horizontal klystron for the XFEL. An alternate design is being developed to improve on the manufacturability and reliability of the MBK. The Sheet Beam Klystron (SBK) has fewer parts and processes than an MBK. It is focused with a periodic permanent magnet (PPM) system and, as a result, is smaller and weighs less than an MBK. 

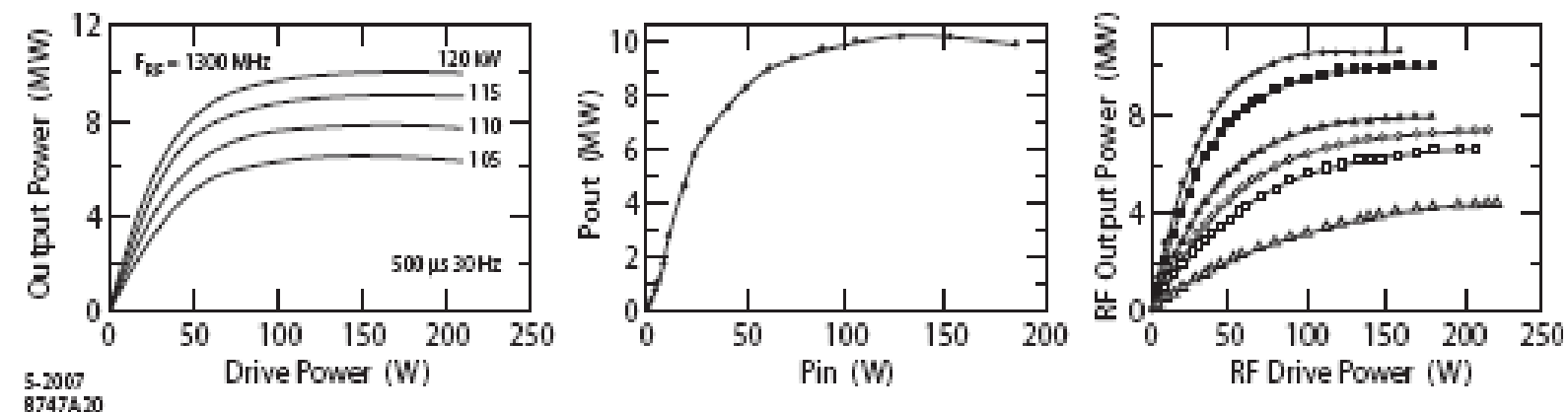

Figure 2.12: Test results for: (a) CPI VKL-8301 at reduced pulse width; (b) Toshiba MBK E3736 at full spec pulse width; (c) Thales TH1801 at reduced pulse width.

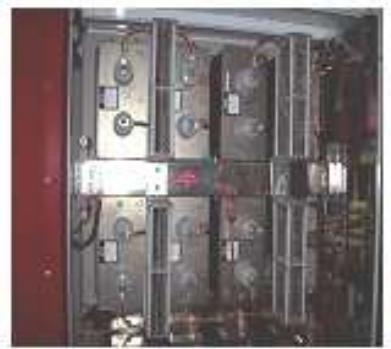

(a)

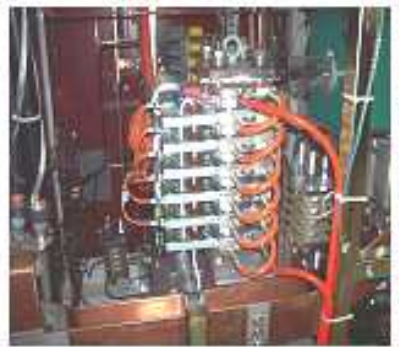

(b)

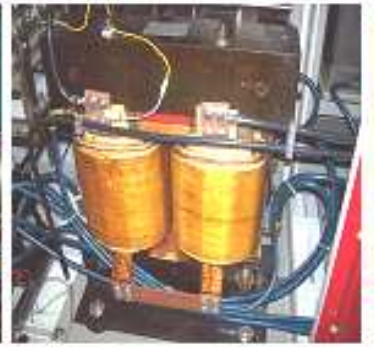

(c)

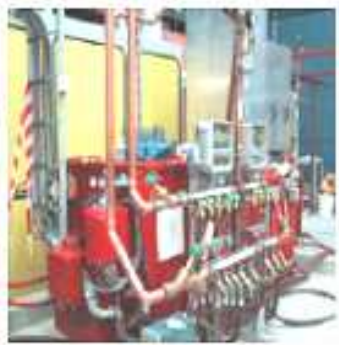

(d)

Figure 2.13: (a) Capacitor Stack, (b) Dual IGBT Switch, (c) Bouncer Choke, (d) Pulse transformer.

DESY is already working with the manufacturers to produce a horizontal klystron for the XFEL. An alternate design is being developed to improve on the manufacturability and reliability of the MBK. The Sheet Beam Klystron (SBK) has fewer parts and processes than an MBK. It is focused with a periodic permanent magnet (PPM) system and, as a result, is smaller and weighs less than an MBK.

\subsubsection{Klystron Modulator}

Modulator operation is straightforward: the charger delivers a DC voltage to the storage capacitors of approximately $11 \mathrm{kV}$. The modulator main switch is then triggered and held closed for about $1.7 \mathrm{msec}$. Capacitor current flows through the switch to the step-up transformer input. At the same time, an auxiliary droop compensation "bouncer" circuit is fired to maintain the pulse top at to within $\sim 0.5 \%$ during the RF drive period. The slightly above $10 \mathrm{kV}$ drive pulse (to compensate for Bouncer voltage) is delivered to the input of the pulse transformer in order to produce at least $115.7 \mathrm{kV}, 133.0$ A to the klystron for rated 10 MW peak output. Principal devices of a klystron modulator are shown in Figure 2.13. 


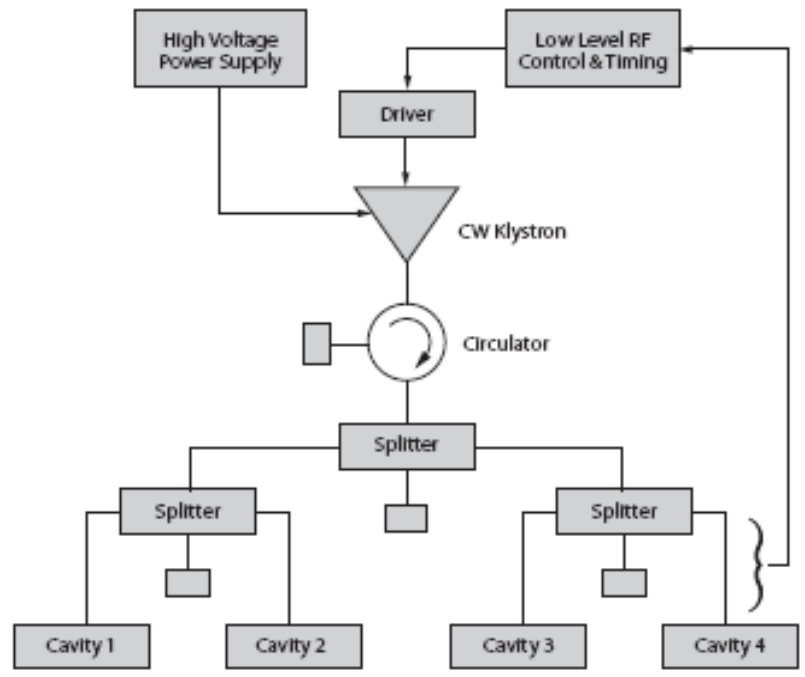

Figure 2.14: Damping Ring 1.2 MW RF station (1 of 20).

\begin{tabular}{|c|c|c|}
\hline Specification & Typical & Maximum \\
\hline Charger input voltage kV RMS & 7.67 & 8 \\
\hline Charger average power input $\mathrm{kW}$ & 147.9 & 161.7 \\
\hline Charger efficiency & 0.93 & 0.93 \\
\hline Charger DC output voltage $=$ Modulator $\mathrm{kV}_{\text {in }}$ & 10.8 & 11.3 \\
\hline Charger DC avg output current $=$ Modulator $A_{\text {in }}$ & 13.26 & 13.26 \\
\hline Charger average power output @ $5 \mathrm{~Hz} \mathrm{~kW}$ & 137.5 & 150.3 \\
\hline Modulator efficiency & 0.94 & 0.94 \\
\hline Modulator pulse voltage output $=$ Pulse Transformer $\mathrm{kV}_{\text {in }}$ & 10.16 & 10.18 \\
\hline Modulator pulse current output $=$ Pulse Transformer $A_{\text {in }}$ & 1560 & 1680 \\
\hline Modulator average power output @ $5 \mathrm{~Hz} \mathrm{~kW}$ & 129.3 & 141.3 \\
\hline Pulse transformer step-up ratio & 12 & 12 \\
\hline Pulse transformer efficiency & 0.97 & 0.97 \\
\hline Pulse transformer voltage out $=$ Klystron $\mathrm{kV}_{\mathrm{pk}}$ & 115.7 & 120 \\
\hline Pulse transformer current out $=$ Klystron $A_{\mathrm{pk}}$ & 133.0 & 140 \\
\hline Pulse transformer average power output $@ 5 \mathrm{~Hz} \mathrm{~kW}$ & 125.4 & 137.1 \\
\hline High voltage pulse duration $(70 \%$ to $70 \%) \mathrm{ms}$ & 1.631 & 1.7 \\
\hline High voltage rise and fall time ( 0 to $99 \%) \mathrm{ms}$ & $<0.23$ & 0.23 \\
\hline High voltage flat top $(99 \%$ to $99 \%) \mathrm{ms}$ & 1.565 & 1.565 \\
\hline Pulse flatness during flat top \% & $< \pm 0.5$ & \pm 0.5 \\
\hline Pulse to pulse voltage fluctuation \% & $< \pm 0.5$ & \pm 0.5 \\
\hline Energy deposit in klystron from gun spark $\mathrm{J}$ & $<20$ & 20 \\
\hline Pulse repetition rate, $\mathrm{Hz}$ & 5 & 5 \\
\hline Klystron filament voltage $\mathrm{V}$ & 9 & 11 \\
\hline Klystron filament current A & 50 & 60 \\
\hline
\end{tabular}

Table 2.10: Modulator Specifications \& Requirements Assuming Klystron $\mu \mathrm{P}=3.38$, Efficiency $=65 \%$. 
The ILC Accelerator Design

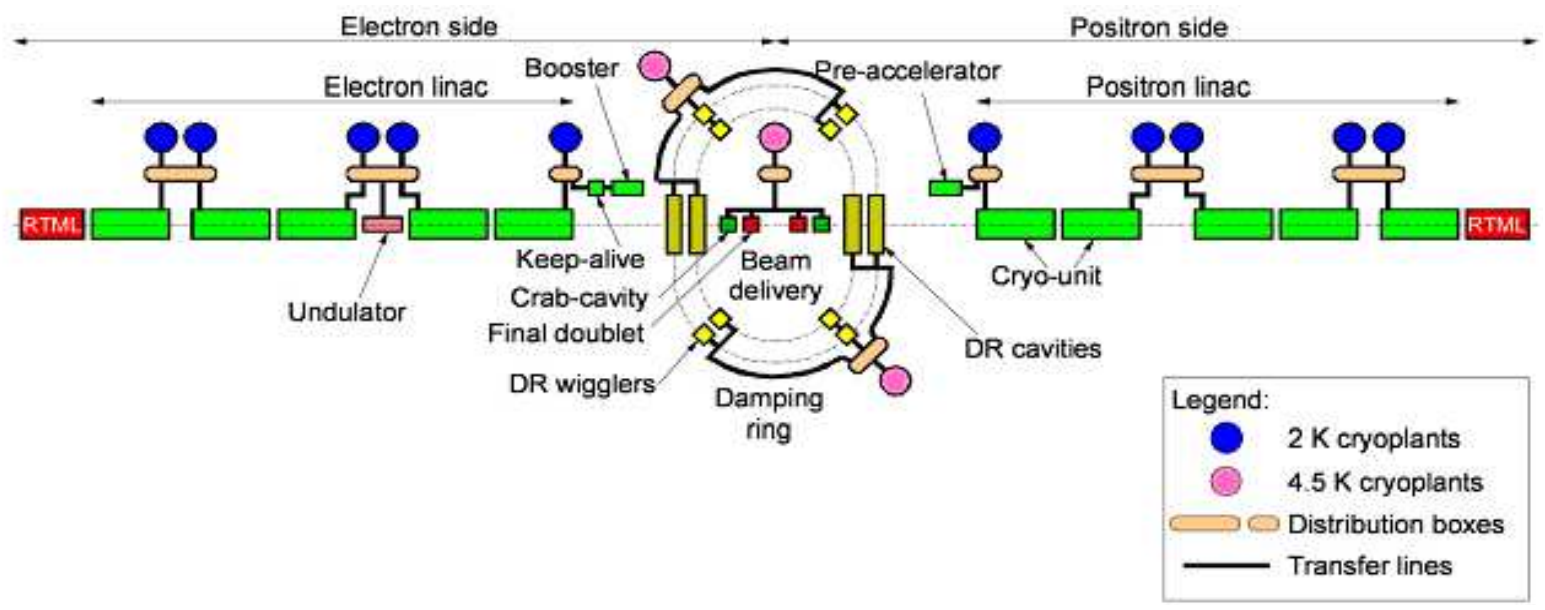

Figure 2.15: The overall layout concept for the cryogenic systems.

The Damping Rings have $650 \mathrm{MHz}$ CW stations using 1.2 MW peak power klystrons, 20 in total for 2 rings. Power is supplied from a DC supply of $2.0 \mathrm{MW}$ delivering $50-75 \mathrm{kV}$ at 17 10 A DC. The RF envelope is controlled by the low level RF and timing to maintain stability and clearing gaps as needed. The station block diagram is shown in Figure 2.14. There are no major technical issues with the L-Band modulator as long as the entire system has sufficient overhead (redundancy) to compensate for a failed station. To achieve an acceptable availability, the linac energy and beam current parameters must be chosen to provide some RF spare stations. Redundancy of internal components such as IGBT switches and sectioning of chargers for $\mathrm{N}+1$ redundancy is also important. Currently this is only partially implemented in the prototypes. The present design which develops the drive pulse at low voltage and high current has larger losses than would be experienced with a higher voltage design. This is not a major technical issue, but a cost, size and weight issue. Installation and repair during operations will be more difficult with multi-ton components such as the transformer and main capacitor switch multi-cabinet assembly. An alternative modulator design is being investigated to address these issues, including the possibility of significant cost reduction. The design would reduce the overall footprint and eliminate the step-up transformer and other oil-filled components.

\subsubsection{The cryogenic systems}

With superconducting equipment throughout the ILC, cryogenic systems of extensive size and capacity will be required (Figure 2.15). Superconducting RF cavities operating at $2 \mathrm{~K}$ in the main linacs are the primary accelerating structures in the ILC and comprise the largest cryogenic cooling load. Although not as extensive, the positron and electron sources, damping rings, RTML, and beam delivery systems include a large number and variety of superconducting RF cavities. 


\begin{tabular}{|l|c|c|c|c|c|c|c|}
\hline $\begin{array}{c}\text { Cryomodules } \\
\begin{array}{c}\text { (cavities/cryomoule) } \\
\text { (quads/cryomodule) } \\
\text { (requency, MHz) }\end{array}\end{array}$ & $\begin{array}{c}8-\mathrm{C} \\
1-\mathrm{Q} \\
1300\end{array}$ & $\begin{array}{c}9-\mathrm{C} \\
0-\mathrm{Q} \\
1300\end{array}$ & $\begin{array}{c}8-\mathrm{C} \\
2-\mathrm{Q} \\
1300\end{array}$ & $\begin{array}{c}6-\mathrm{C} \\
6-\mathrm{Q} \\
1300\end{array}$ & $\begin{array}{c}\text { Total } \\
1300\end{array}$ & $\begin{array}{c}1-\mathrm{C} \\
650\end{array}$ & $2-\mathrm{C}$ \\
\hline \hline Main Linac e $^{-}$ & 282 & 564 & & & 846 & & \\
\hline Main Linac e $^{+}$ & 278 & 556 & & & 834 & & \\
\hline RTML e $^{-}$ & 18 & 30 & & & 48 & & \\
\hline RTML e $^{+}$ & 18 & 30 & & & 48 & & \\
\hline $\mathrm{e}^{-}$source & 24 & & & & 24 & & \\
\hline $\mathrm{e}^{+}$booster & 12 & & 6 & 4 & 22 & & \\
\hline $\mathrm{e}^{+}$Keep Alive & 2 & & & & 2 & & \\
\hline $\mathrm{e}^{-}$Damping Ring & & & & & & 18 & \\
\hline $\mathrm{e}^{+}$Damping Ring & & & & & & 18 & \\
\hline Beam Delivery System & & & & & & & 2 \\
\hline \hline Total & 634 & 1180 & 6 & 4 & 1824 & 36 & 2 \\
\hline
\end{tabular}

Table 2.11: Superconducting RF modules in the ILC, excluding the two 6-cavity energy compressor cryomodules located in the electron and positron LTRs.

Table 2.11 summarizes the numbers of various types of superconducting RF modules in the ILC. In addition to the RF modules listed in Table 2.11, there are a variety of superconducting (SC) magnets in the ILC. About one third of the $1.3 \mathrm{GHz}$ cryogenic modules contain SC magnets. As part of the positron source, the electron linac includes about 150 meters of SC helical undulators in 2 to 4 meter length units. The Damping Rings have 8 strings of superconducting wiggler magnets, and there are special SC magnets in the sources, RTML, and beam delivery system. Figure 2.15 illustrates the concept for the cryogenic system arrangement in ILC. Ten large cryogenic plants with 2 Kelvin refrigeration cool the main linac, RTML and the electron and positron sources. Three smaller cryogenic plants with mostly $4.5 \mathrm{~K}$ loads cool the damping rings and beam delivery system. The ILC cryogenic systems are defined to include cryogen distribution as well as production. Thus, components of the cryogenic system include the cryogenic plants, distribution and interface boxes, transfer lines, and non-magnetic, non-RF cold tunnel components. Although cryomodules, SC magnets, and production test systems also include significant cryogenics, those are not considered in this section of the RDR. Main linac cryogenic modules each containing eight (with magnet package) or nine (without magnet package) nine-cell niobium cavities, cold helium pipes, and thermal shields are the dominant load to be cooled by the cryogenic system. The magnet package, in one third of the cryomodules, includes a superconducting quadrupole and corrector magnets. The ILC cryomodule design for the $1.3 \mathrm{GHz}$ RF is based on the TESLA Test Facility (TTF) type III design which contains all the cryogenic pipe-work inside its vacuum enclosure. There are approximately $23 \mathrm{~km}$ of $1.3 \mathrm{GHz}$ cryomodules including main linac, RTML, and sources. Series architecture is mostly used in the cryogenic unit cooling scheme. 


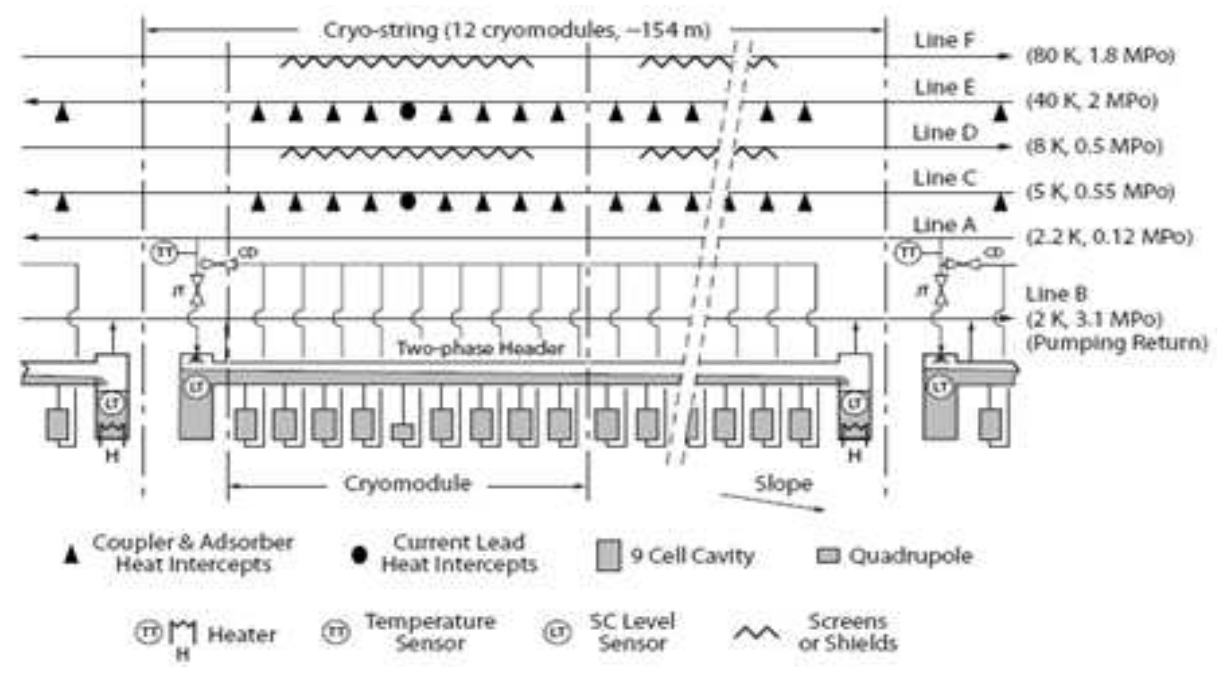

Figure 2.16: Cooling scheme of a cryo-string.

Like for the TESLA cryogenic concept, saturated He II cools RF cavities at $2 \mathrm{~K}$, and helium gas cooled shields intercept thermal radiation and thermal conduction at $5-8 \mathrm{~K}$ and at $40-80$ K. A two-phase line (liquid helium supply and concurrent vapor return) connects to each helium vessel and connects to the major gas return header once per module. A small diameter warm-up/cool-down line connects the bottoms of the He vessels. A subcooled helium supply line connects to the two-phase line via a Joule-Thomson valve once per string (typically 12 modules). The $5 \mathrm{~K}$ and $40 \mathrm{~K}$ heat intercepts and radiation screens are cooled in series through an entire cryogenic unit of up to $2.5 \mathrm{~km}$ in length. For the $2 \mathrm{~K}$ cooling of the RF cavities, a parallel architecture is implemented with the parallel cooling of cryo-strings resulting in operational flexibility. Consequently, each cryogenic unit is subdivided into about 14 to 16 cryo-strings, each of which corresponds to the 154 meter length elementary block of the cryogenic refrigeration system. Figure 2.16 shows the cooling scheme of a cryo-string, which contains 12 cryomodules. The cavities are immersed in baths of saturated super-fluid helium gravity filled from a $2 \mathrm{~K}$ two phase header. Saturated super-fluid helium owes all along the two-phase header for filling the cavities and phase separators located at both ends of the twophase header. The first phase separator is used to stabilize the saturated liquid produced during the final expansion. The second phase separator in used to recover the excess of liquid, which is vaporized by a heater. At the interconnection of each cryomodule, the twophase header is connected to the pumping return line. The division of the Main Linac into cryogenic units is driven by various plant size limits and a practical size for the low pressure return pipe. A cryogenic plant of $25 \mathrm{~kW}$ equivalent $4.5 \mathrm{~K}$ capacity is a practical limit due to industrial production for heat exchanger sizes and over-the-road shipping size restrictions. Cryomodule piping pressure drops also start to become rather large with more than $2.5 \mathrm{~km}$ distances. Practical plant size and gas return header pressure drop limits are reached with 192 modules in a 16-string cryogenic unit, $2.47 \mathrm{~km}$ long. 


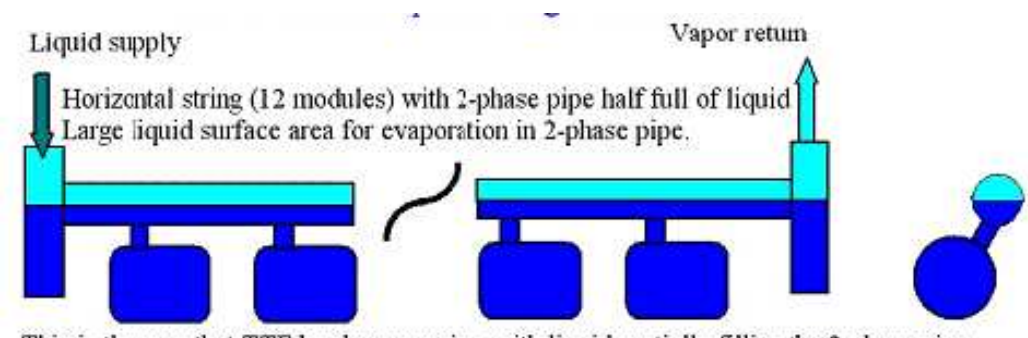

This is the way that TTF has been running, with liquid partially filling the 2-phase pipe.

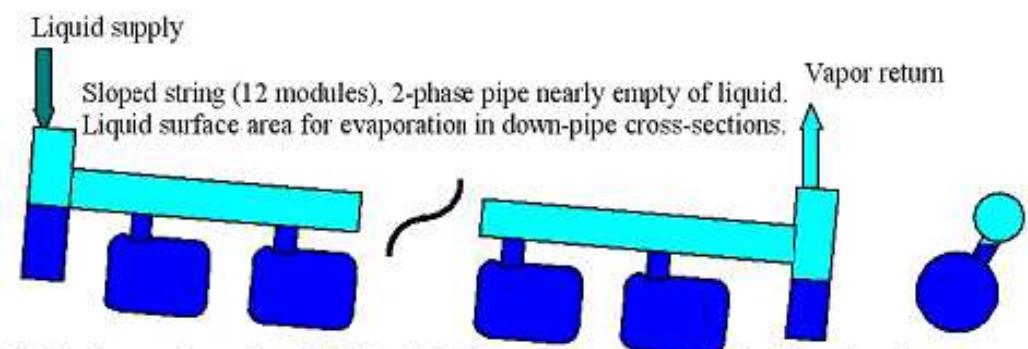

This is the way that a sloped IC (and XFEL) would operate, with liquid running down the 2-phase pipe, filling each helium vessel, and excess liquid dropping into a vessel at the string end where it is boiled away by a small electric heater.

Figure 2.17: Two-phase helium flow for level and for sloped systems.

Five cryogenic units divide the main linac conveniently for placing the positron source undulators at $150 \mathrm{GeV}$. As the ILC site has not yet been selected, the cryogenic system concept must accommodate different configurations of tunnel and civil works. The tunnel may follow the earth's curvature or be laser-straight with a maximum slope of up to $0.6 \%$ creating large elevation differences. To avoid harmful instabilities, all fluid should ideally be transported over large distances in a mono-phase state. Local two-phase circulation of saturated liquid can be tolerated over limited lengths, within a controlled range of vapor quality. Figure 2.17 illustrates two methods of liquid management in the two-phase supply pipe for main linac cryogenic modules, one case for a sloped system and the other for a level system. As listed above in Table 2.10, electron and positron sources each include just over 20 SRF modules containing 1.3 GHz RF cavities cooled to 2 Kelvin. The sources also include several superconducting magnets, as well as about 150 meters of superconducting positron source undulators. These undulators are cooled by one of the cryogenic plants in the electron linac cryogenic system. Damping ring cryogenic loads include $4.5 \mathrm{~K}$ superconducting wigglers, $4.5 \mathrm{~K} 650 \mathrm{MHz}$ cryomodules, associated cryogenic distribution systems, and $70 \mathrm{~K}$ thermal shields for all of these. Two cryogenic plants serve the damping rings. The beam delivery system has one $3.9 \mathrm{GHz}$ cryomodule (containing two cavities) on each side of the interaction point, superconducting final focus quadrupoles, and other special superconducting magnets spaced several hundred meters from the IR. One cryogenic plant serves both sides of the interaction region. 
The ILC Accelerator Design

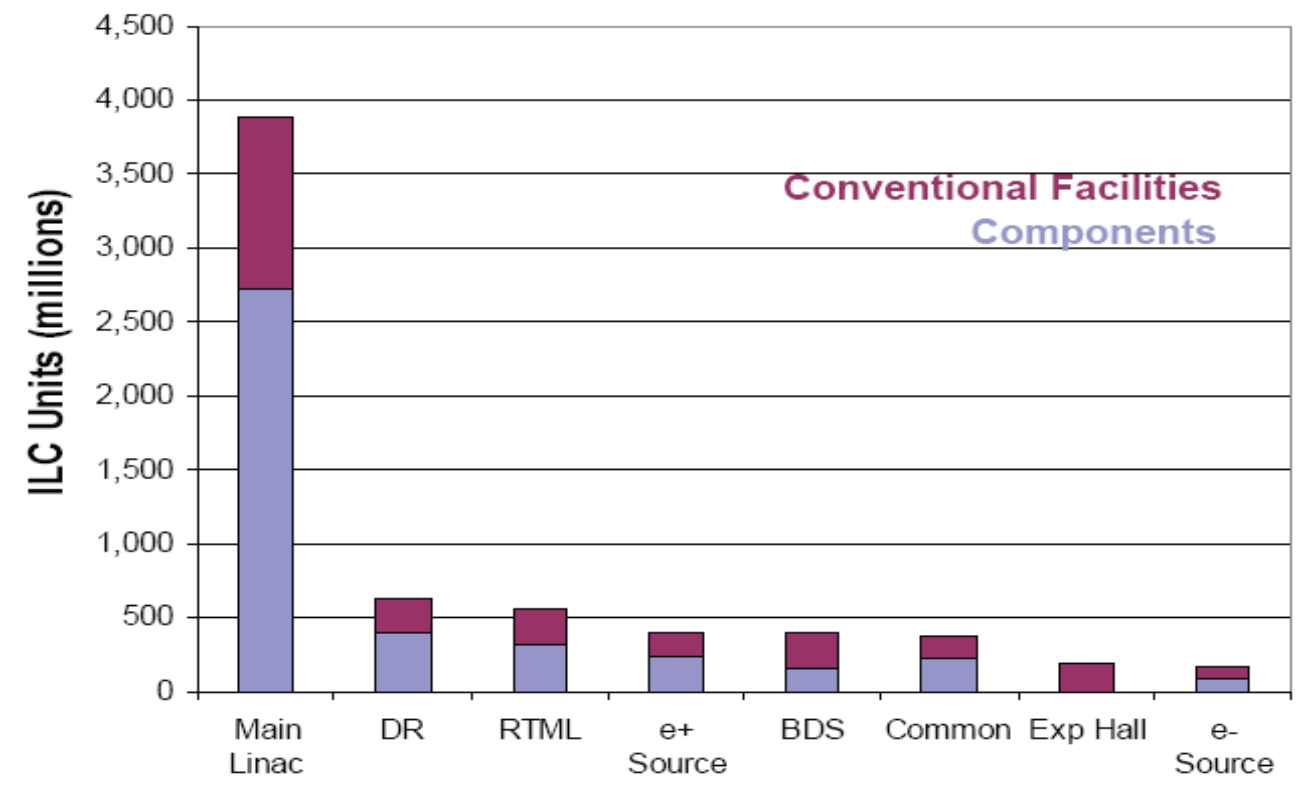

Figure 2.18: Distribution of the ILC value estimate by area system and common infrastructure, in ILC Units. The estimate for the experimental detectors for particle physics is not included.

\subsection{Estimate for construction of ILC}

The value and explicit labor estimates are current as of February 1, 2007, and will be updated in the final report. The preliminary value is for the cost of the ILC in its present design and at the present level of engineering and industrialization. The estimate contains three elements:

- 1.83 Billion (ILC Units) for site-dependent costs, such as the costs for tunneling in a specific region;

- 4.79 Billion (ILC Units) for shared value of the high technology and conventional components;

- 14,200 person-years for the required supporting manpower (= 24 million personhours)

A common estimate was used for all non site-specific technical components, regardless of region. The three regional site-specific estimates were based on local costs for civil engineering and the primary high voltage electrical power connections, feeds, substations and primary cooling water systems. All three site-dependent estimates are within a few percent of the average. There are many possible models for dividing the responsibilities among the collaborating regions. The numbers below present one possible model where the estimates are divided into site-specific and shared parts. In this model, the host region is expected to provide the site-specific parts, because of the size, complexity, and specific nature of these elements. 
The ILC Accelerator Design

\begin{tabular}{|c|c|c|c|}
\hline Region & Site-Specific & Shared & Total \\
\hline \hline Asia & $1.75 \mathrm{~B}$ & $4.78 \mathrm{~B}$ & $6.53 \mathrm{~B}$ \\
\hline Americas & $1.89 \mathrm{~B}$ & $4.79 \mathrm{~B}$ & $6.68 \mathrm{~B}$ \\
\hline Europe & $1.85 \mathrm{~B}$ & $4.79 \mathrm{~B}$ & $6.64 \mathrm{~B}$ \\
\hline and Average & $1.83 \mathrm{~B}$ & $4.79 \mathrm{~B}$ & $6.62 \mathrm{~B}$ \\
\hline \hline \multicolumn{5}{|c|}{ plus 14 K person-years of explicit labor } \\
or 24 M person-hours 1,700 hours/year \\
\hline
\end{tabular}

Table 2.12: Possible division of responsibilities for the 3 sample sites (ILC Units).

The site-specific elements include all the civil engineering (tunnels, shafts, underground halls and caverns, surface buildings, and site development work); the primary high-voltage electrical power equipment, main substations, medium voltage distribution, and transmission lines; and the primary water cooling towers, primary pumping stations, and piping. Responsibilities for the other parts of the conventional facilities: low-voltage electrical power distribution, emergency power, communications, HVAC, plumbing, fire suppression, secondary water-cooling systems, elevators, cranes, hoists, safety systems, and survey and alignment, along with the other technical components, could be shared between the host and non-host regions. Such a model may be summarized as shown in Table 2.12. The value estimates broken down by Area System are shown separately for both the conventional facilities and the components in Figure 2.18 and Table 2.13. Common refers to infrastructure elements such as computing infrastructure, high-voltage transmission lines and main substation, common control system, general installation equipment, site-wide alignment monuments, temporary construction utilities, soil borings and site characterization, safety systems and communications. The component value estimates for each of the Area (Accelerator) Systems include their respective RF sources and cryomodules, cryogenics, magnets and power supplies, vacuum system, beam stops and collimators, controls, Low Level RF, instrumentation, installation, etc.. The superconducting RF components represent about $69 \%$ of the estimate for all non- CF\&S components. Initial cursory analysis of the uncertainties in the individual estimates from the Technical Systems indicates that the RMS for the current RDR value estimate for the presented baseline design is likely to be in the $\sigma= \pm 10-15 \%$ range, and that the 95th percentile for this estimate is no larger than $+25 \%$ above the mean. The explicit labor for the Global Systems, Technical Systems, and specific specialty items for Electron Source, Positron Source, Damping Rings, and Ring to Main Linac, include the scientific, engineering, and technical staff needed to plan, execute, and manage those elements including specification, design, procurement oversight, vendor liaison, quality assurance, acceptance testing, integration, installation oversight, and preliminary check-out of the installed systems. 


\begin{tabular}{|l|c|c|c|}
\hline Area - M ILC Units & Total & Components & $\begin{array}{c}\text { Conventional } \\
\text { Facilities }\end{array}$ \\
\hline \hline Main Linac & 3,894 & 2,723 & 1,172 \\
\hline DR & 630 & 398 & 231 \\
\hline RTML & 554 & 320 & 234 \\
\hline $\mathrm{e}^{+}$source & 398 & 232 & 166 \\
\hline BDS & 408 & 157 & 252 \\
\hline Common & 369 & 229 & 140 \\
\hline Exp Hall & 200 & 0 & 200 \\
\hline $\mathrm{e}^{-}$source & 165 & 87 & 78 \\
\hline \hline Sum & 6,618 & 4,146 & 2,472 \\
\hline
\end{tabular}

Table 2.13: Distribution of the ILC Value Estimate by area system and common infrastructure, in ILC Units. The estimate for the experimental detectors for particle physics is not included.

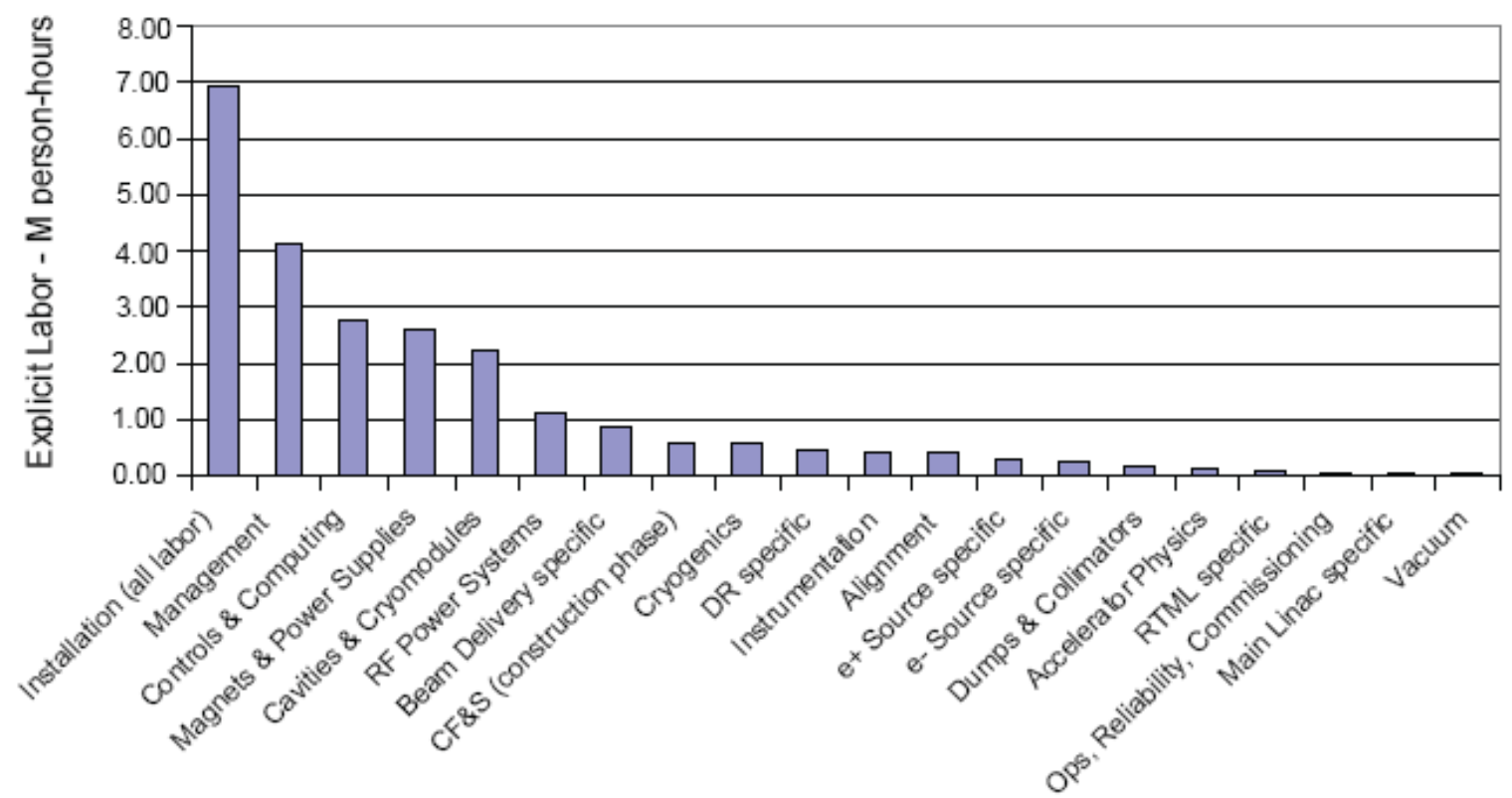

Figure 2.19: Explicit labor, which may be supplied by collaborating laboratories or institutions listed by Global, Technical, and some Area-specific Systems. 


\begin{tabular}{|l|c|}
\hline Explicit labor & M person-hours \\
\hline \hline Installation (all labor) & 6.91 \\
\hline Management & 4.09 \\
\hline Controls \& computing & 2.76 \\
\hline Magnets \& Power Supplies & 2.60 \\
\hline Cavities \& cryomodules & 2.23 \\
\hline RF Power Systems & 1.12 \\
\hline Beam Delivery System specific & 0.89 \\
\hline CF\&S (construction phase) & 0.60 \\
\hline Cryogenics & 0.56 \\
\hline DR specific & 0.46 \\
\hline Instrumentation & 0.44 \\
\hline Alignment & 0.42 \\
\hline$e^{+}$Source specific & 0.31 \\
\hline$e^{-}$Source specific & 0.24 \\
\hline Dumps \& Collimators & 0.19 \\
\hline Accelerator Physics & 0.11 \\
\hline RTML specific & 0.09 \\
\hline Ops, Reliability, Commissioning & 0.07 \\
\hline Main Linac specific & 0.06 \\
\hline Vacuum & 0.05 \\
\hline Sum & 24.19 \\
\hline
\end{tabular}

Table 2.14: Explicit labor, which may be supplied by collaborating laboratories or institutions, listed by Global, Technical, and some Area-specific Systems.

Installation is the largest fraction of explicit labor, about 29\%. Management is the second largest fraction at about $17 \%$. At this stage of the ILC design, it is too early for a complete analysis of installation requirements. Instead, the RDR estimate was based on scaled information from a variety of sources, including the actual manpower used for the installation of recent accelerator projects. There was also a bottoms-up study for installation of the cryomodules for the Main Linac done by two separate engineering teams, with comparable results. The estimates were reviewed by experts and crosschecked for reasonability. In the present estimate, the installation task is characterized almost exclusively as explicit labor, with minimum costs for material-handling equipment. This is on the assumption that much of the installation and system check-out labor at the ILC site can be contributed by the staffs of collaborating institutions or laboratories. The validity of this assumption depends on the availability of the necessary skilled manpower and local labor regulations. Because of the size of the project, it is likely that many tasks like electrical and plumbing work will need to be outsourced to industry. 


\begin{tabular}{|c|c|}
\hline Unit & Responsibilities \\
\hline Directorate (30): & $\begin{array}{l}\text { Directors Office, Planning, ES\&H Over- } \\
\text { sight, Legal, External Affairs, Educa- } \\
\text { tion, International Coordination, Technol- } \\
\text { ogy Transfer; }\end{array}$ \\
\hline Management Division (13): & Quality Assurance, ES\&H; \\
\hline Laboratory Technical Services (125): & $\begin{array}{l}\text { Facilities Services, Engineering Support, } \\
\text { Material and Logistical Services, Labora- } \\
\text { tory Fabrication Shops, Staff Services; }\end{array}$ \\
\hline Administrative Services (94): & $\begin{array}{l}\text { Personnel, Finance, Procurement, Minor- } \\
\text { ity Affairs; }\end{array}$ \\
\hline Project Management Division (83): & $\begin{array}{l}\text { Management, Administrative, Project } \\
\text { Management Division Office. }\end{array}$ \\
\hline
\end{tabular}

Table 2.15: Composition of the management structure at ILC.

Trade-offs and translations are likely between using in-house labor and external contracts. It is estimated that a minimum of $10 \%$ of the installation task must be management and supervision by in-house manpower. The ILC staff consists of 345 persons, divided as shown in Table 2.15. Personnel for the Area, Global, and Technical System groups are not included in the Project Management Division. This explicit labor estimate is very preliminary. Producing a more realistic explicit labor estimate will be a priority in the Engineering Design phase. It is the practice in some regions to apply general and administrative overheads to purchases and labor for projects. These overheads are applied as a multiplier on the underlying LABOR and VALUE, and cover the costs of the behind-the-scenes support personnel. In this estimate, such personnel are explicitly enumerated as labor under Directorate, Management Division, Laboratory Technical Service, and Administrative Services in Table 2.15. Therefore, the overheads are included as additional explicit LABOR, rather than as a multiplier on VALUE. This explicit labor corresponds to $35 \%$ scientists and engineers, 14\% administrative personnel, 27\% technical staff, and 24\% installation staff which could be either institutional or laboratory labor or contract labor or some combination. Operating costs are not included in the estimate for the construction project, but a very preliminary estimate is given. It is also to be noted that spare components (those stored in warehouses and not the installed redundant components), although fabricated along with the installed components, are assumed to be financed through operating funds, and are not considered part of the construction projects. Major factors in the operating cost include personnel costs, electrical power, maintenance and repairs, helium and nitrogen consumables, and components that have a limited life expectancy and need continuous replacement or refurbishment, like klystrons. The model assumes 9 months of machine operations per year at full power of about $227 \mathrm{MW}$, corresponding to $500 \mathrm{GeV}$ at design luminosity, plus 3 months standby at reduced power (25 MW) with the superconducting cavities maintained at $4.5 \mathrm{~K}$, which is above their operating temperature. At the current electrical power rate of $\$ 0.1$ per $\mathrm{kW}-\mathrm{hr}$, the operating costs for these materials and services are estimated to be approximately 150-270 M\$ per year in 2007 Dollars. 


\section{Chapter 3}

\section{Klystron Modulator Power Supplies}

\subsection{Modulator charging PS design requirements}

The baseline design for the International Linear Collider, outlined in the Reference Design Report [8], envisions two parallel tunnels for each main linac, an accelerator tunnel housing the beamline itself and a service tunnel containing the RF sources - power supplies, modulators, and klystrons - that power it through waveguide penetrations. A primary goal of the current ILC design phase is to identify and evaluate cost-saving options. The Modulators are used to generate the pulsed power for the klystrons of $1.3 \mathrm{GHz}$ Radio Frequency superconducting cavities of the International Linear Collider. They have been able to produce rectangular high voltage pulses of with amplitude of $120 \mathrm{kV}$ corresponding a nominal current of $120 \mathrm{~A}, 2 \mathrm{~ms}$ of pulse width and a repetition rate of $10 \mathrm{~Hz}$. Besides the droop of high voltage pulses must be around $1 \%$ of nominal value. The electrical power during the pulse is typically $15 \mathrm{MW}$ and can be at maximum $16.8 \mathrm{MW}$. This leads to a needed pulsed power of 8.9 GW. It is obvious that this energy can not be taken from the mains directly. Therefore it is stored in capacitor banks to be released during the pulse. DC/DC switching power supply is needed to recharge the main capacitor banks and to decouple the low repetition rate from the mains.

General requirements of a DC/DC switching power supply to the charge the klystron modulators are mentioned as follows:

- Maximum charge voltage $=12 \mathrm{kV}$;

- Pulse repetition rate $=10 \mathrm{~Hz}$;

- Average power per modulator $=300 \mathrm{~kW}$;

- Power factor $24 \%$;

- Total line voltage variation at $5 \div 10 \mathrm{~Hz}= \pm 0.5 \%$;

- Maximum efficiency;

- The Main Capacitor Bank (MCB) loaded with constant power, minimum ripple, phase distortions and an accuracy of $\pm 0.5 \%$;

- Maximum power factor of the rectifier, feeding the charge device by both decreasing the harmonic composition of the consumed current and by the high value of $\cos \varphi$;

- The low repetition rate $(5 \div 10 \mathrm{~Hz})$ has to be suppressed in order not to produce disturbances to the mains. 

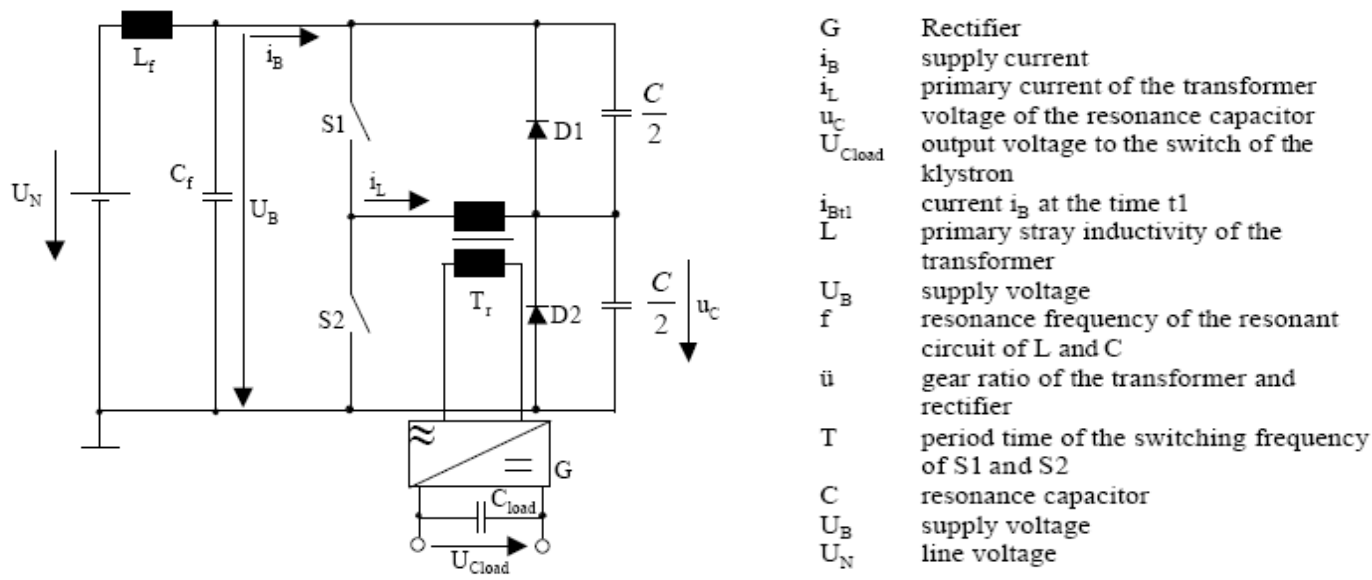

Figure 3.1: Shematic of the series resonance sine converter.

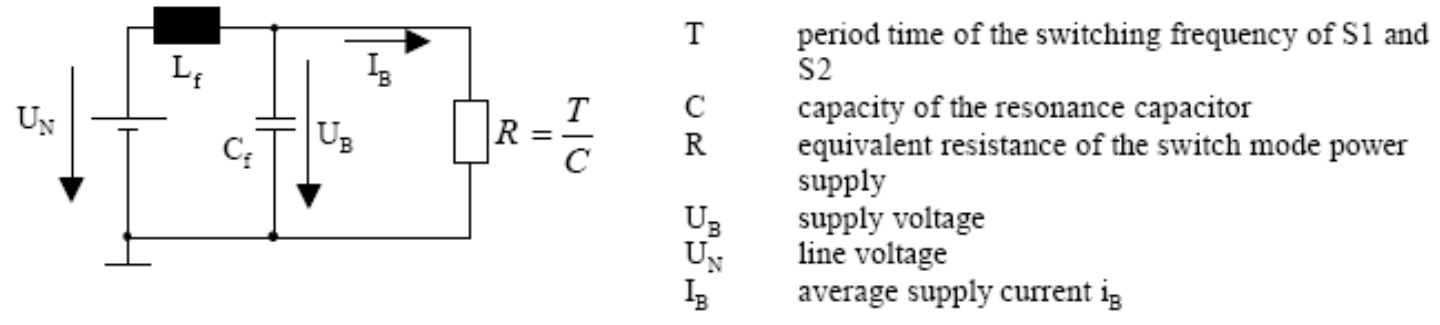

Figure 3.2: Equivalent circuit of the power supply.

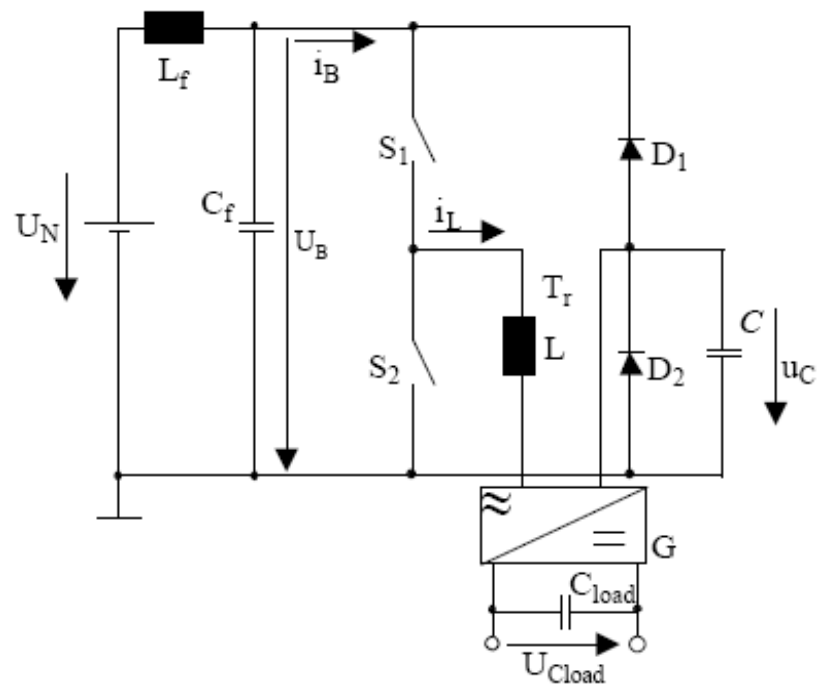

Figure 3.3: Series resonance sine converter with only one capacitor 


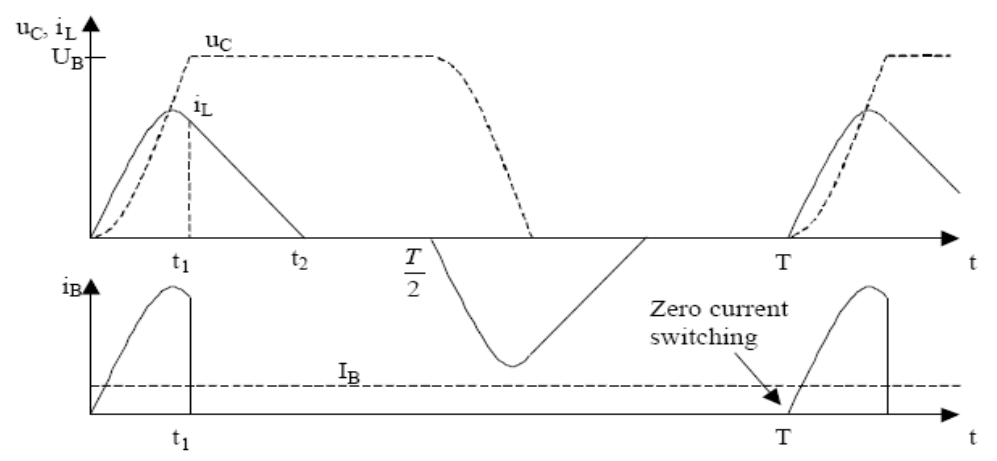

Figure 3.4: Voltage and current function of the half bridge.

\subsection{Modulator power supply design analysis}

\subsubsection{TESLA design}

The power supply shown in Figure 3.1 was developed at DESY [12]. This topology is known for small power supplies for auxiliary voltages. So far it has not been used as power supply to charge large capacitive loads in a constant power mode. The equivalent circuit of the switch mode power supply (Figure 3.2) is a resistor $\mathrm{R}$ which is constant when the period time $\mathrm{T}$ of the switching frequency $\mathrm{f}(10-20 \mathrm{kHz})$ is constant. This resistance is independent of the capacitor voltage $\mathrm{U}_{\mathrm{Cload}}$ and the pulse repetition rate of the modulator. By this the input power is (in a wide range) independent from the voltage of the main capacitor bank of the modulator. To study the circuit in Figure 3.1 we can consider the circuit in Figure 3.3.

Assumption: $\mathrm{u}_{\mathrm{c}}=0 \mathrm{~V}, \mathrm{I}=0 \mathrm{~A}$ and $\mathrm{S}_{1}$ turning on. Because of the stray inductance $\mathrm{L}$ of the transformer the current $i_{\mathrm{L}}$ keeps zero while turning $\mathrm{S}_{1}$ on (zero current switching power supply).Then the current $i_{L}$ raises and the resonance capacitor $C$ is charged. While charging the resonance capacitor $C$ the supply current $i_{B}$ is equivalent to the resonance capacitor current $\mathrm{i}_{\mathrm{L}}$. When the resonance capacitor voltage $\mathrm{u}_{\mathrm{c}}$ reaches the supply voltage UB diode $\mathrm{D}_{1}$ will start to conduct. The current $\mathrm{i}_{\mathrm{L}}$ continues flowing forced by the energy stored in the stray inductance $\mathrm{L}$ of the transformer. Since the path of the current $i_{\mathrm{L}}$ is no longer through the resonance capacitor $C$ and the supply current $i_{B}$ equals zero. The energy stored in the stray inductance $\mathrm{L}$ of the transformer will slowly be passed to the load Cload and the current $\mathrm{i}_{\mathrm{L}}$ decreases linearly to zero. After that a new cycle can start by turning $\mathrm{S}_{1}$ off and $\mathrm{S}_{2}$ on. The corresponding voltage and current waveforms can be seen in Figure 3.4. With each loading and unloading period the same amount of energy is transmitted from the primary side of the transformer to the main capacitor bank. This amount is the energy of the resonance capacitor loaded to the voltage $\mathrm{UB}_{\mathrm{B}}$ or unloaded to 0 . 


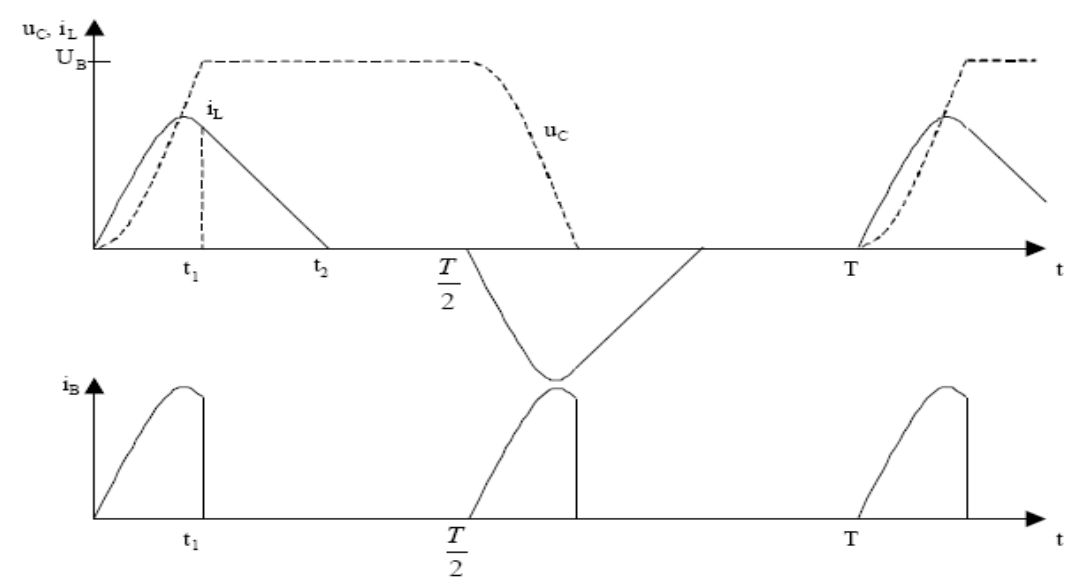

Figure 3.5: Voltage and current waveform of power supply.

While charging the resonance capacitor $C$ the supply current $i_{B}$ is equivalent to the resonance capacitor current $\mathrm{i}_{\mathrm{L}}$. Therefore the charge $\mathrm{Q}$ taken from the power supply to the resonance capacitor C equals $Q=C U_{B}$. The charge $\mathrm{Q}$ can also be expressed as follows:

$$
Q=\int_{0}^{T} i_{B} d t=U C_{B}
$$

Multiplication of the integral with the term $T / T$ with $\mathrm{T}=$ period time of the switching time:

$$
Q=T *\left[\int_{0}^{T} i_{B} d t\right]=C U_{B}
$$

The term in brackets is equivalent to the average supply current $I_{B}=\int_{0}^{T} i_{B} d t$.

$$
Q=T * I_{B}=C U_{B}
$$

The resulting expression for the average supply current is:

$$
I_{B}=\frac{C U_{B}}{T}=\frac{U_{B}}{R}
$$

The voltage and current wave forms for the half bridge shown in Figure 3.4 are given in Figure 3.5. The same principle as for the half bridge is valid. The difference is that the 


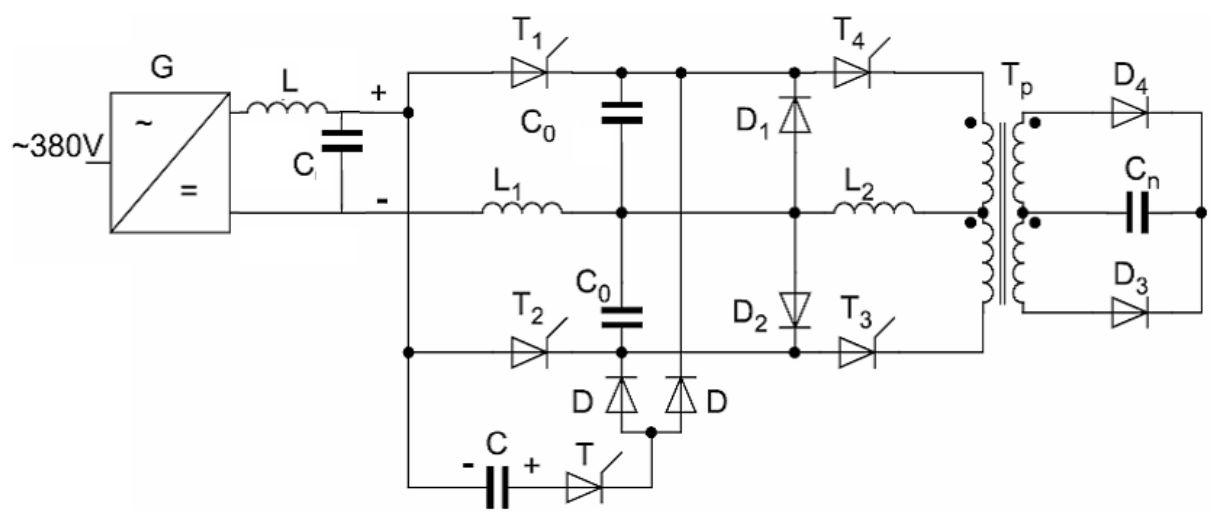

Figure 3.6: Simplified circuit diagram of a charge device with an electron protection.

frequency of the current it doubled and the currents are decreased. When one of the capacitors is loaded, the second capacitor is unloading and vice versa. The required power is with today's technique hard to fulfill with just one power part. A $300 \mathrm{~kW}$ prototype consisting of four modules having $75 \mathrm{~kW}$ each is going to be tested in TTF. One $75 \mathrm{~kW}$ module was tested successfully.

\subsubsection{A Russian design for TESLA}

The basic part of the module is a thyristor inverter converting the input voltage into current pulses at a high repetition rate, charging in portions the capacitor up to the required voltage through the step-up transformer (Figure 3.6). The inverter consists of two loops. The first loop consist of a main rectifier $G$, thyristors $T_{1}$ and $T_{2}$, a choke $L_{1}$ and dosing capacitors $C_{0}$, the second loop includes capacitors $\mathrm{C}_{0}$, thyristors $\mathrm{T}_{3}$ and $\mathrm{T}_{4}$, diodes $\mathrm{D}_{1} \div \mathrm{D}_{4}$ and a storage capacitor $\mathrm{C}_{\mathrm{n}}$. The step-up transformer $\mathrm{T}_{\mathrm{p}}$ used only to transform the inverter output voltage. The inverter works in the following way. Capacitors $\mathrm{C}_{0}$ are charged in succession from a lowvoltage mains rectifier $G$ up to its two-fold voltage by switching on thyristors $T_{1}$ and $T_{2}$ through the inductance $\mathrm{L}_{1}$. Every half-period of the inverter operation they transfer their energy to the capacitor $C_{n}$, being discharged by switching $T_{3}$ and $T_{4}$ through the inductance $\mathrm{L}_{2}$ to the primary winding of the transformer $\mathrm{T}_{\mathrm{p}}$. For a complete energy transfer from $\mathrm{C}_{0}$ to $\mathrm{C}_{\mathrm{n}}$ they are shunted by diodes $\mathrm{D}_{1}$ and $\mathrm{D}_{2}$. With the help of these diodes there is no over-charging of the capacitors $\mathrm{C}_{0}$, the energy is completely transferred to capacitor $\mathrm{C}_{\mathrm{n}}$ and there are provided initial conditions for the next charge cycle. The last process is the most principle one in the inverter operation. W e have situation, when the rectifier inside the charge interval works to dosing capacitors with zero initial conditions: $\mathrm{Uc}_{0}=0$. But the capacitors $\mathrm{C}_{0}$ are discharged completely with a full energy transfer to the storage capacitor $\mathrm{C}_{\mathrm{n}}$ only to the value $U c_{n}=U_{G} \cdot n\left(U_{G}=500 \mathrm{~V}\right.$ is the voltage at the rectifier, $n$ is the transformation ratio of the transformer $\mathrm{T}_{\mathrm{p}}$ ). 
With a further increase in the voltage on $\mathrm{C}_{\mathrm{n}}$, the capacitors $\mathrm{C}_{0}$ don't discharge completely, which leads to a reduction in the average value of the consumed current and a violation of the constant power consumption from the mains. The capacitor charging is finished at $U c_{n} \sim$ $(1.05 \div 1.1) U_{G} \cdot n$, which is the maximum tolerable over-voltage in the circuit. Thus, the range $0<U c_{n}<U_{G} \cdot n$ is the condition for the optimal operation at a constant power consumption. The control of the charge voltage level is performed by varying the pulse repetition rate of the inverter. The maximum pulse repetition rate corresponds to the continuous inverter operation and the maximum charge voltage. The voltage decreases with the pulse repetition rate. The thyristors are triggered by means of a functional "voltage-frequency" converter which is controlled with the help of a variable reference voltage. The operation of a single charging device, provided the mean power consumed from the mains is constant, is characterized by ripples with drops to zero in the intervals between the charge pulses. At a maximum repetition rate the phase shift between the sinusoidal charge pulse of the current is equal to $\pi$. In this case, the efficiency coefficient $K_{e f}=I_{a v} / I_{\max }$ ( $\mathrm{I}_{\max }$-maximum value of current) is equal to 0.637 and decrease with the increase in the phase shift during the voltage drop in the process of control. The multi phase design of the charge system decreases fluctuations and makes the efficiency coefficient equal to $\mathrm{K}_{\mathrm{ef}}=0.9$ in the two-phase variant for the phase shift by $\pi / 2,0.95$ and 0.97 in the three - and four - phase variants, respectively. The inverter doesn't require an insulating mains transformer in the rectifier G; Either one, or a number $(10 \div 20)$ of inverters can operate from one high-current rectifier. Between the rectifier and the inverter an electron switch is used. It works together with emergency switches which are of a comparatively slow action. The protection circuit operates as follows: the capacitor C, charged from the low power rectifier $(50 \div 100 \mathrm{~W})$ ( in the indicated polarity, is in a waiting mode. During the alarm situation, for example, when the operation fails or inverter sticks, the rectifier current feeding the inverter goes out of the design mode. In this moment, when it reaches the determinate level, the thyristor $\mathrm{T}$ is switched on by a comparator signal and the reverse voltage from the capacitor $C$ is applied o the inverter thyristors $T_{1}$ and $T_{2}$ at the same time the trigger pulses are taken off. The thyristor which was switched on at that moment $\left(\mathrm{T}_{1}\right.$ or $\mathrm{T}_{2}$ ), switches off, the inverter alarm current is taken over by the circuit: the capacitor $\mathrm{C}$, the thyristor $\mathrm{T}$ and one of diodes $\mathrm{D}$. After the polarity reversal of the $\mathrm{C}$, the thyristor $\mathrm{T}$ is switched off and the protection circuit is restored. It seems advantageous to employ the following scheme of a power supply for the TESLA modulators:

- Each inverter is located near the modulator. It is connected to the rectifier through contactor;

- The contactor is remote-controlled; in emergency situations it is automatically disconnected.

Many of charge devices are based on thyristor rectifiers, where the capacitor charge mode is preset by a thyristor switch phase. As a rule, the thyristor switch phase is close to $\pi$ at the beginning of the charge interval and as the capacitor is being charged, it decrease, approaching at the best value of the mains phase, i.e. $\pi / 3$. This fact impairs the harmonic composition of the consumed current and leads to a charge of $\cos \varphi$ inside the charge interval. 


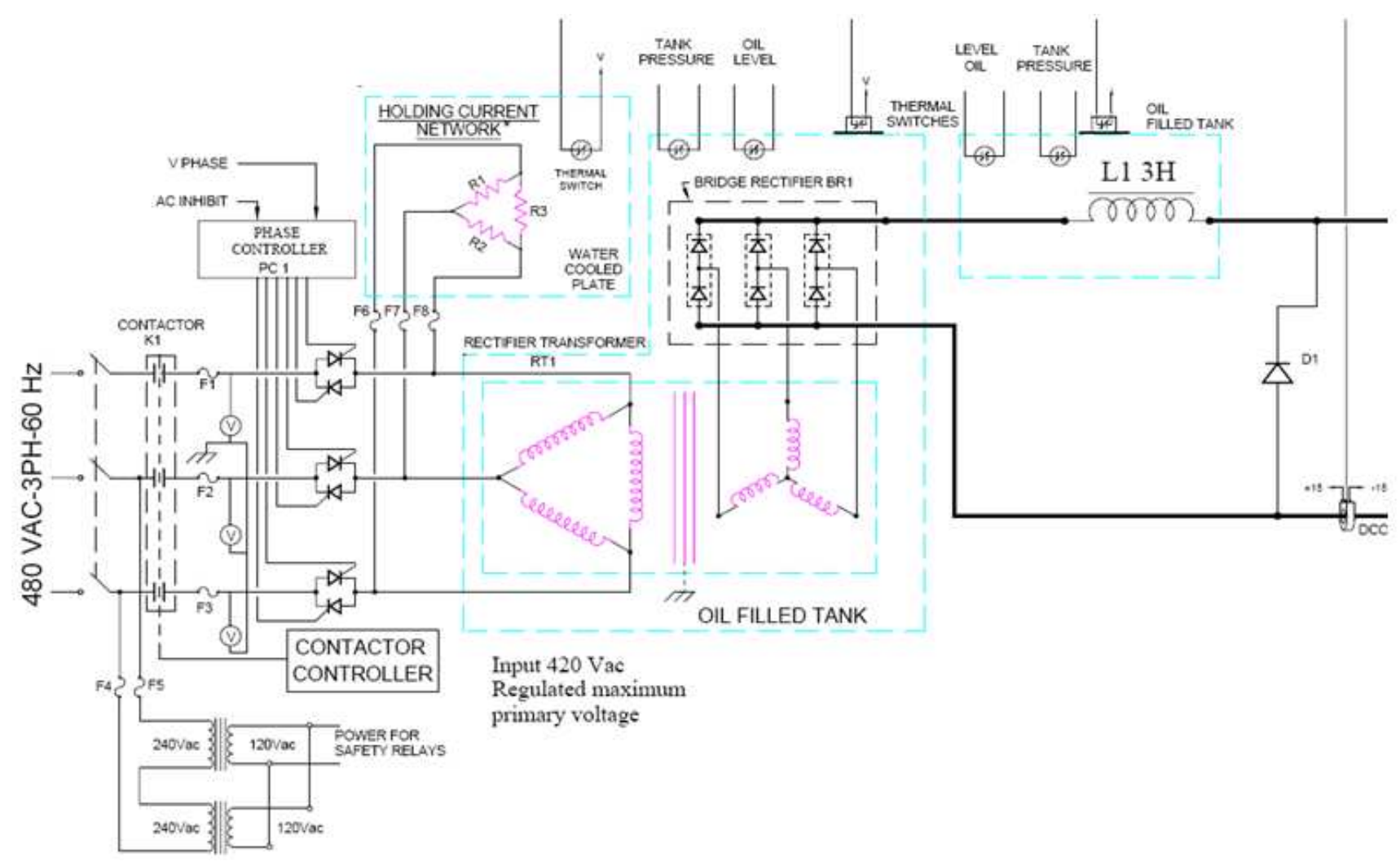

Figure 3.7: Fermilab DC power supply $10.6 \mathrm{kV} @ 14 \mathrm{~A}$.

The charge systems with thyristor regulators reduce the rectifier power factor. It is known that the rectifier power factor $\chi$ depends on the shift factor $\cos (\varphi)$ as well as on the distortion factor $v$ :

$$
\chi=v * \cos \varphi
$$

So some disadvantages of the charge system described are:

- The charge device is based on the inverter, that is, as a powerful frequency converter, a source of radio interference voltage. That is why it is necessary to connect an interference filter at its input. The industry produces a great number of interference filters for different currents and voltages with a working damping of 60,80 and 100 $\mathrm{dB}$;

- The inverter is a source of noises of the sound range; the main sources of noises are the chokes $\mathrm{L}_{1}, \mathrm{~L}_{2}$ and the transformer $\mathrm{T}_{\mathrm{p}}$. We partially decrease this disadvantage placing these elements in a tank filled with transformer oil. 


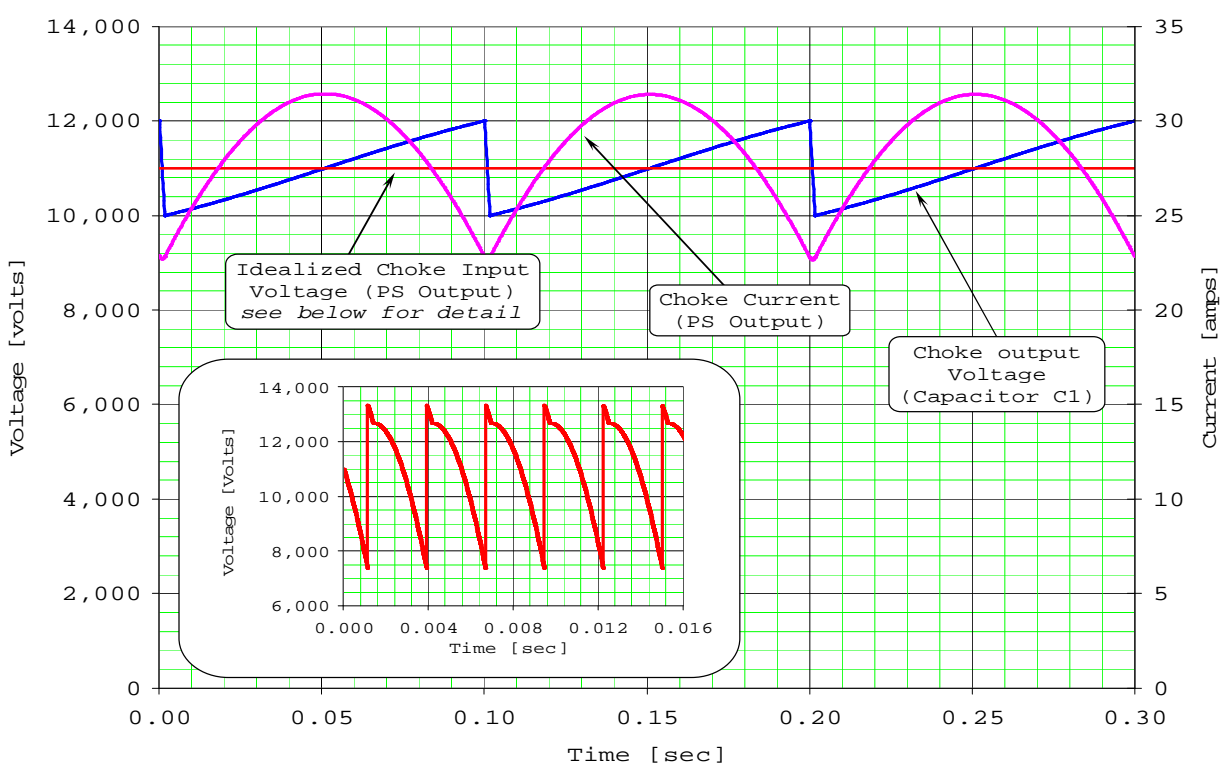

Figure 3.8: PS Voltage / Current and Capacitor Voltage.

\subsubsection{Fermilab power supply}

The Figure 3.7 represents the Fermilab modulator DC power supply now under test in a ILC test facility called Meson Area.

The principal parts of the DC power supply are:

- A rectifier transformer, composed by a step-up transformer to increase the voltage primary (420 Vac) with a three-phase rectifier non controlled bridge to straighten the current waveform;

- A big choke to level the charging current at $5 \mathrm{~Hz}$ with these specifications:

- 3 Henries $\pm 7 \%$ at $60 \mathrm{~Hz}$;

- Less than $3 \mathrm{~kW}$ total losses under the operating conditions;

- A current of 28.5 Amps RMS maximum, 32 Amps peak;

- No less than $90 \%$ of nominal inductance at 36 Amps.

- A primary phase controller to regulate the operating voltage.

The advantages of this power supply are:

- Simplicity;

- Relative low cost; 


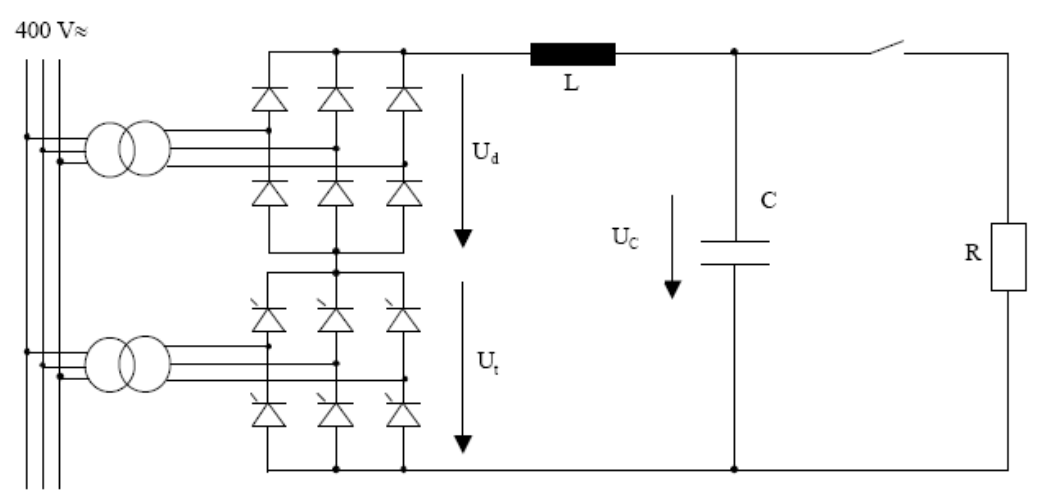

Figure 3.9: Series connection of a diode and a SCR bridge.

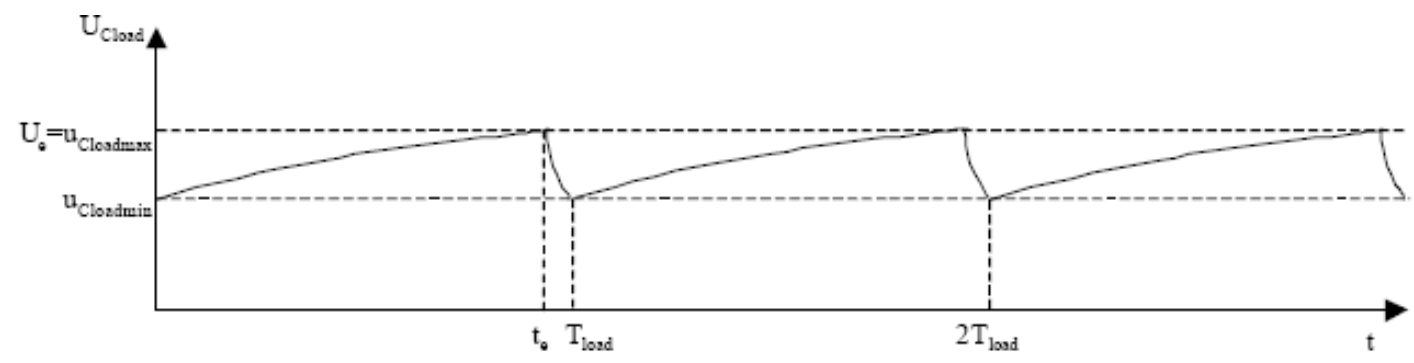

Figure 3.10: Voltage curve form of the main capacitor bank in the modulator.

In other hand the disadvantages of the device are :

- The necessity to have an holding current network for the SCRs use;

- The burden and the price of the large 3H choke;

- The high variation of the power supply output current waveform as shown in Figure 3.8 ;

\subsubsection{SCR series with a diode rectifier}

When we looks at the waveform of the capacitor bank, the modulator voltage (Figure. 3.10) can be divided into two parts. There is a constant DC part and a part of varying voltage. The basic idea is to produce the constant DC part with an unregulated diode rectifier. The changing part will be produced by a switched mode power supply e.g. a buck converter. By this combination the full regulation dynamic of the switched mode supply can be used. Since the price of the diode rectifier is app. $40 \%$ to $50 \%$ of the price of a switched mode supply, an overall price reduction of $20 \%$ to $30 \%$ seems possible depending on the chosen value of the DC voltage. 


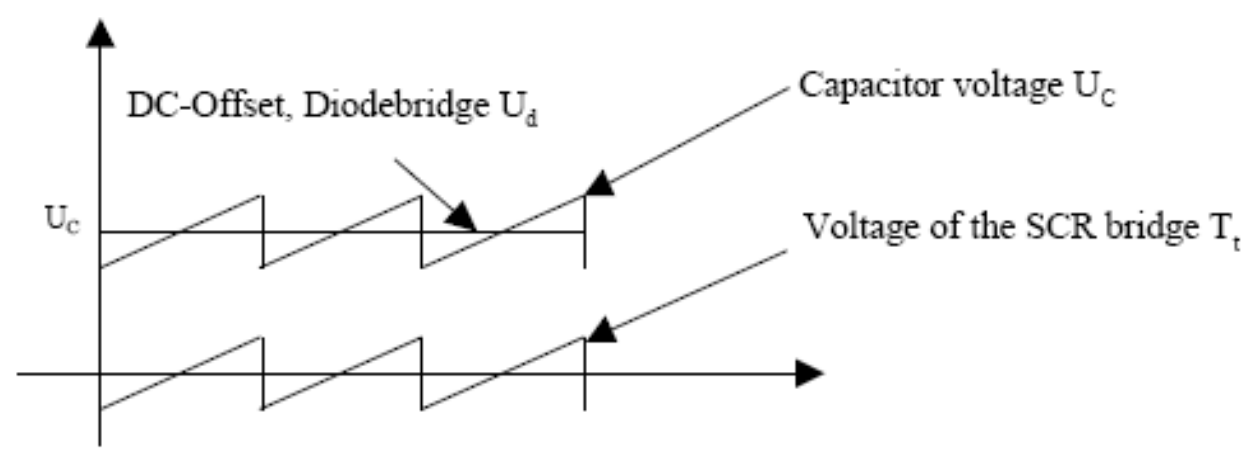

Figure 3.11: Voltage curve of series connection of diode and SCR Bridge.

The same basic idea as for the hybrid supply is assumed. Here the switched mode supply shall be replaced by SCR technology to further reduce cost. The voltage of the diode rectifier is at $10 \mathrm{kV}$. The SCR has to be in inverter mode at the beginning of the loading period to decrease the voltage. At the end of the loading period the power supply has to add voltage. A principle schematic is shown in Figure. 3.9. The voltage curves are shown in Figure. 3.11.

\subsubsection{SCR bridge with a single phase control}

The power of the SCR supply can be calculated:

P: real power

Q: reactive power

S: apparent power

$\mathrm{Q}_{1}$ : fundamental reactive power

$\mathrm{Q}_{0}$ : harmonics reactive power

$\mathrm{S}_{1}$ : fundamental apparent power

$\alpha$ : trigger angle

$$
P=U_{d i} * I_{L} * \cos \alpha
$$

$$
Q=\sqrt{Q_{1}^{2}+Q_{0}^{2}}
$$

$$
S=\sqrt{P^{2}+Q^{2}}
$$

$$
Q_{1}=U_{d i} * I_{L} * \sin \alpha
$$

$$
Q_{0}=0.31 * U_{d i} * I_{L}
$$

$$
S_{1}=U_{d i} * I_{L}
$$




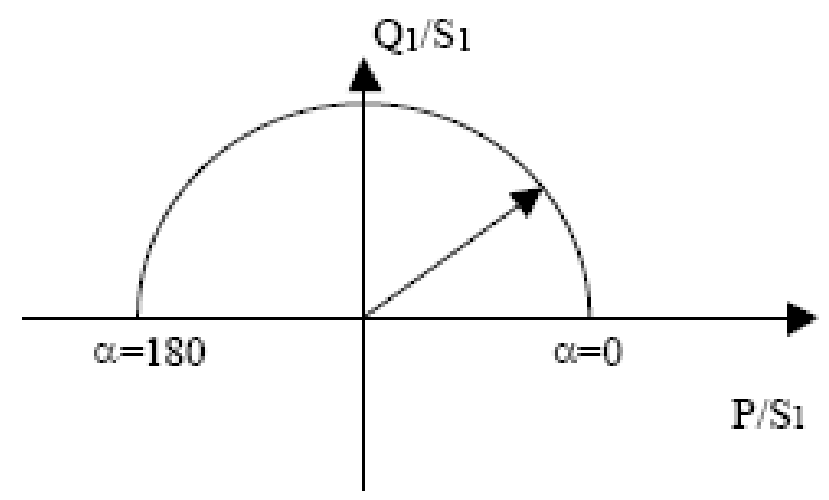

Figure 3.12: Real and reactive power in dependence of the phase angle $\alpha$.

The correspondence of the reactive power to the real power in dependence of the trigger angle is shown in Figure 3.12.

The overall real power is: $P=P_{\text {thyristor }}+P_{\text {diode }}=U_{\text {dit }} * I_{L} * \cos \alpha+U_{d} * I_{L}$

For a constant power $\alpha$ can be calculated: $\alpha=\operatorname{Arccos}\left(\left(\frac{P}{I_{L}}-U_{d i d}\right) * \frac{1}{U_{d i t}}\right)$

The arccos function is only valid when $\frac{P}{I_{L}}-U_{d i d} * \frac{1}{U_{d i t}}$ is between -1 and 1 .

If the relation:

$$
\frac{P}{U_{d i d}+U_{d i t}} \leq I_{L} \leq \frac{P}{U_{d i d}-U_{d i t}}
$$

is not valid, no phase shift angles can be calculated for a constant power mode.

The simulation shows that a constant power mode is possible when having a nominal voltage of $3 \mathrm{kV}$ of the SCR supply. The power diagram is shown in Figure 3.13. The power was calculated in periods of $20 \mathrm{~ms}$ the repetition rate is $10 \mathrm{~Hz}$.

\subsubsection{Digital regulation}

The regulation is a major part of a constant power supply. The voltage of the capacitor bank at the trigger time of the pulses has to have a pulse to pulse repetition accuracy of $+/-0.5 \%$. This in combination with the demand of constant power requires a digital regulation. To be able to react on variations of the mains, temperature effects or non linear behavior of components the regulation is self learning. A simplified modulator circuit is shown in Figure 3.14. The regulator contains a RAM wherein the charging curve of the capacitor voltage $\mathrm{U}_{\text {Cload }}$ is stored. It is driven according to the RAM curve. A fast regulator ensures that the 


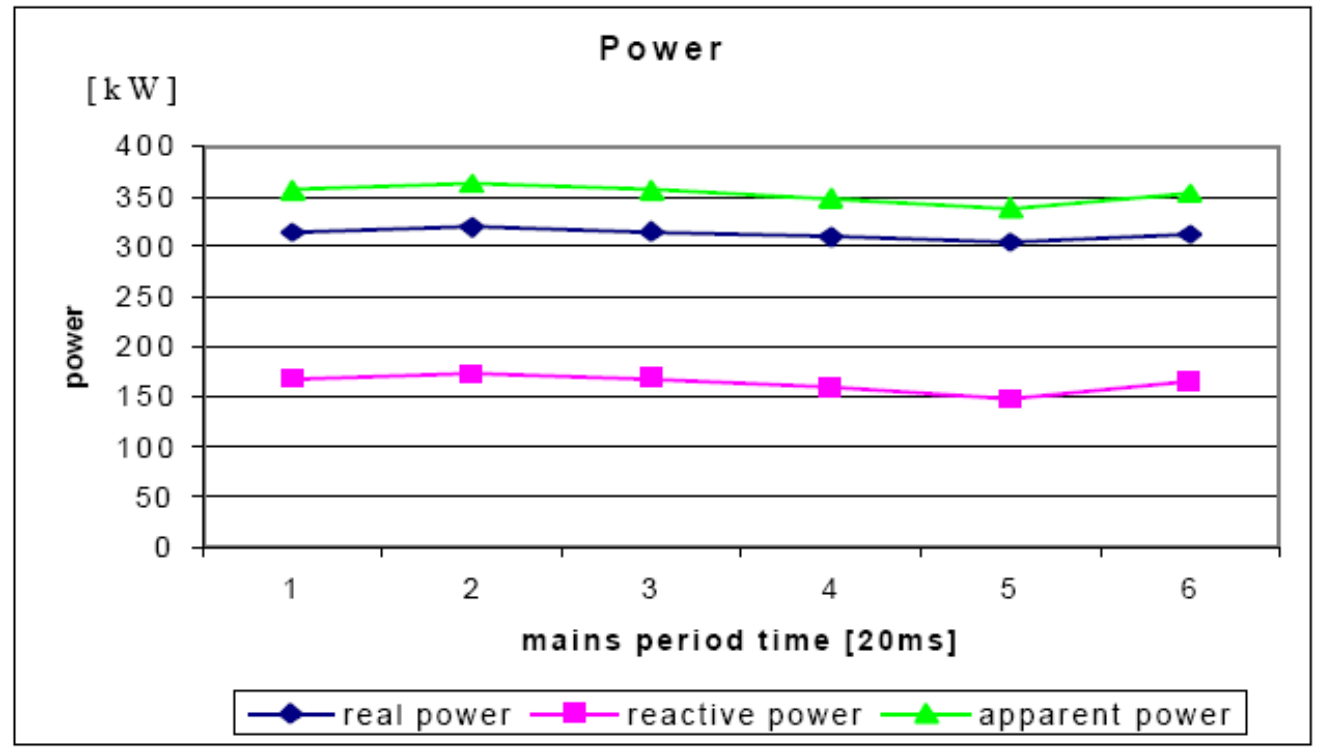

Figure 3.13: Power diagram of a series connection of a diode and SCR bride $\alpha(\mathrm{P})$.

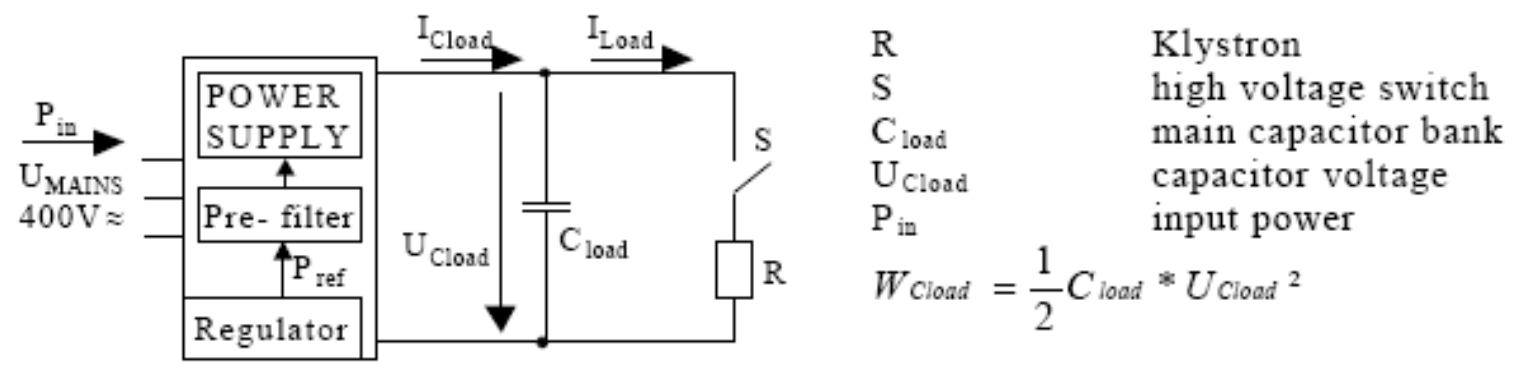

Figure 3.14: Simplified modulator circuit. 


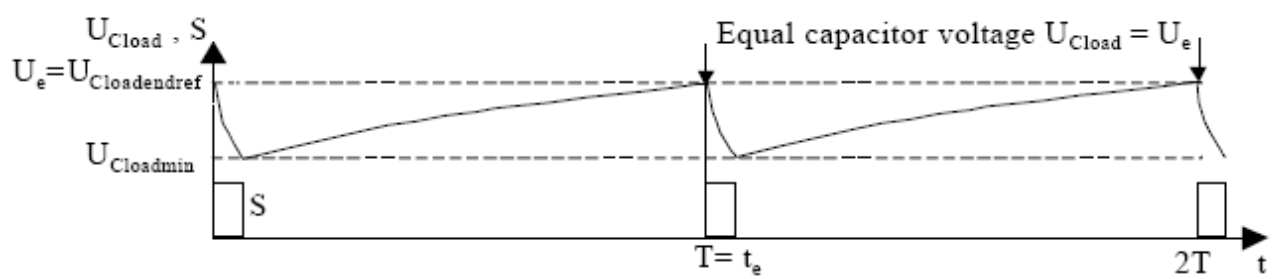

Figure 3.15: Voltage curve of the main capacitor.

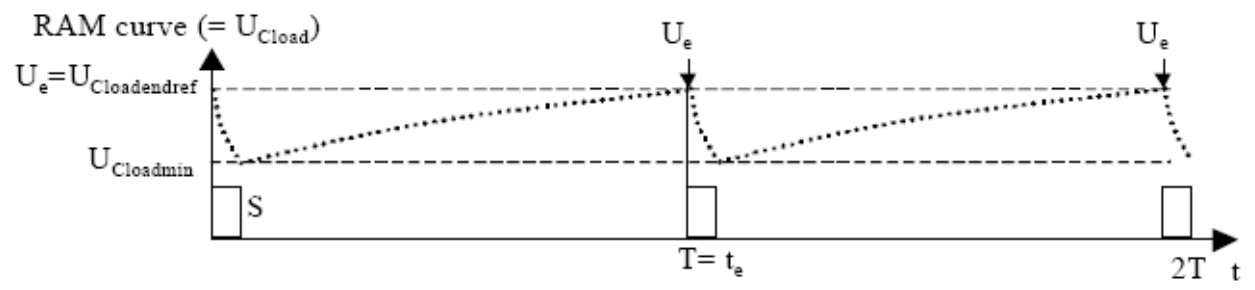

Figure 3.16: Stored reference charging curve of the capacitor.

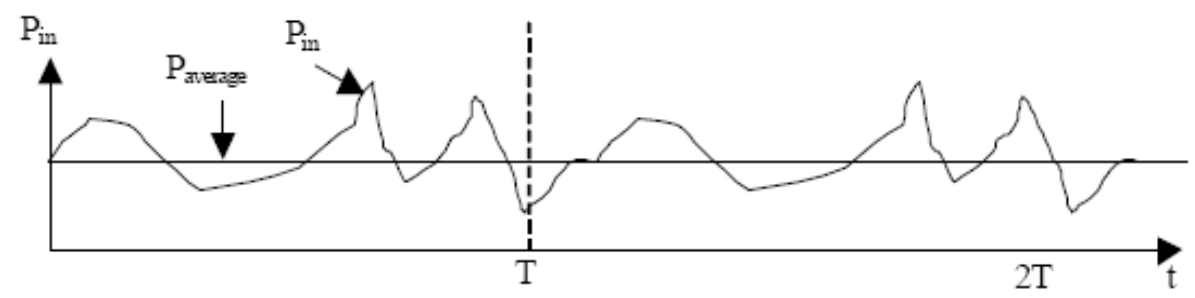

Figure 3.17: Input power of the modulator.

RAM curve and the capacitor voltage $\mathrm{U}_{\text {Cload }}$ are equal despite of even fast voltage variations of the mains voltage $U_{\text {MAINS }}$. For this reason it is obvious that the final voltage $U_{e}=U_{\text {Cload }}$ at time te remains the same at each charging cycle because it equals the well known RAM curve. Figure 3.15 shows again the voltage curve of the main capacitor bank.

For achieving constant input power a learning process is introduced. A voltage charging curve for $\mathrm{C}_{\text {load }}$ is determined. With this curve the final charging voltage equals the nominal voltage $\left(\mathrm{V}_{\text {capend }}=\mathrm{V}_{\text {capendref }}\right)$. This charging curve is stored in the memory. The possible starting curve is shown in Figure 3.16. With this reference charging curve the input power is not yet constant but looks e.g. like the curve shown in Figure 3.17. The aim is to have a constant power. With the self learning algorithm the stored reference curve is modified in such a way that the reference values are increased or decreased until the input power is constant. The result of the learning process is equal output voltage $\mathrm{U}_{\text {Cload }}=\mathrm{U}_{\mathrm{e}}=$ constant after each charging cycle $\mathrm{T}$ and constant input power consumption $\mathrm{P}_{\mathrm{in}}=\mathrm{P}_{\text {average }}=$ constant. 

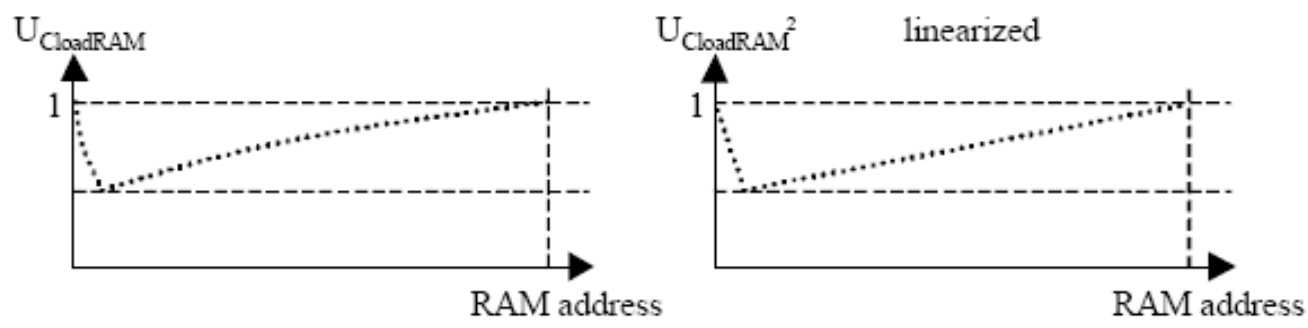

Figure 3.18: RAM curves of the capacitor reference curves.

Reference curve

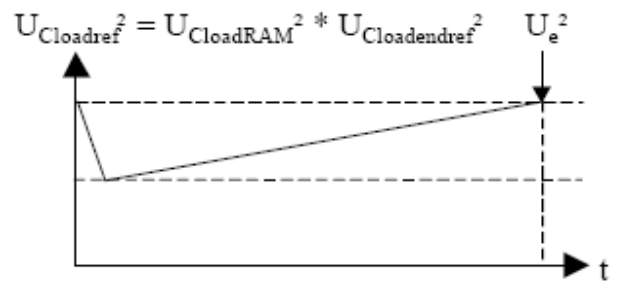

Actual curve

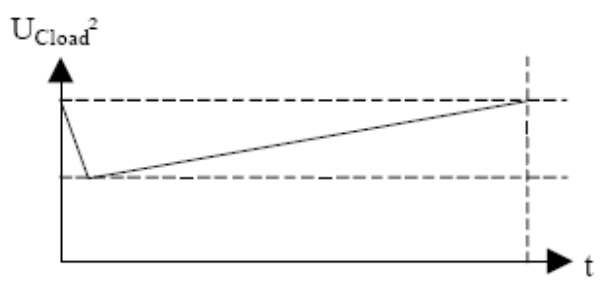

Figure 3.19: Linearized and scaled reference curve and monitored curve.

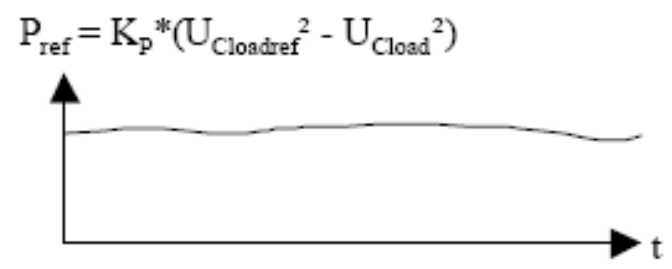

Figure 3.20: Output signal of the regulation.

To be independent from changes in the charging time $\mathrm{T}$ (changes in repetition rate) or the output voltage $\mathrm{U}_{\mathrm{e}}$ a unit curve is stored in the RAM. By this it is not necessary to relearn this curve in case of change. The unit charging curve is simply scaled to the real time and voltage axis. To linearize the regulation the capacitor voltages are used as squared values. This is because the energy stored in the capacitor $\mathrm{C}_{\mathrm{Load}}$ is proportional to the squared capacitor voltage $\mathrm{U}_{\text {Cload }}{ }^{2}$. The linearized curve is stored as RAM curve.

$$
W_{\text {cload }}=\frac{1}{2} C_{\text {load }} * U^{2} \text { cload }
$$

$\mathrm{U}_{\text {Cloadref }}^{2}(\mathrm{t})$ (reference curve) is the linearized reference value of the squared capacitor voltage $\mathrm{U}_{\text {Cload }}^{2}(\mathrm{t})$ (actual curve) . 


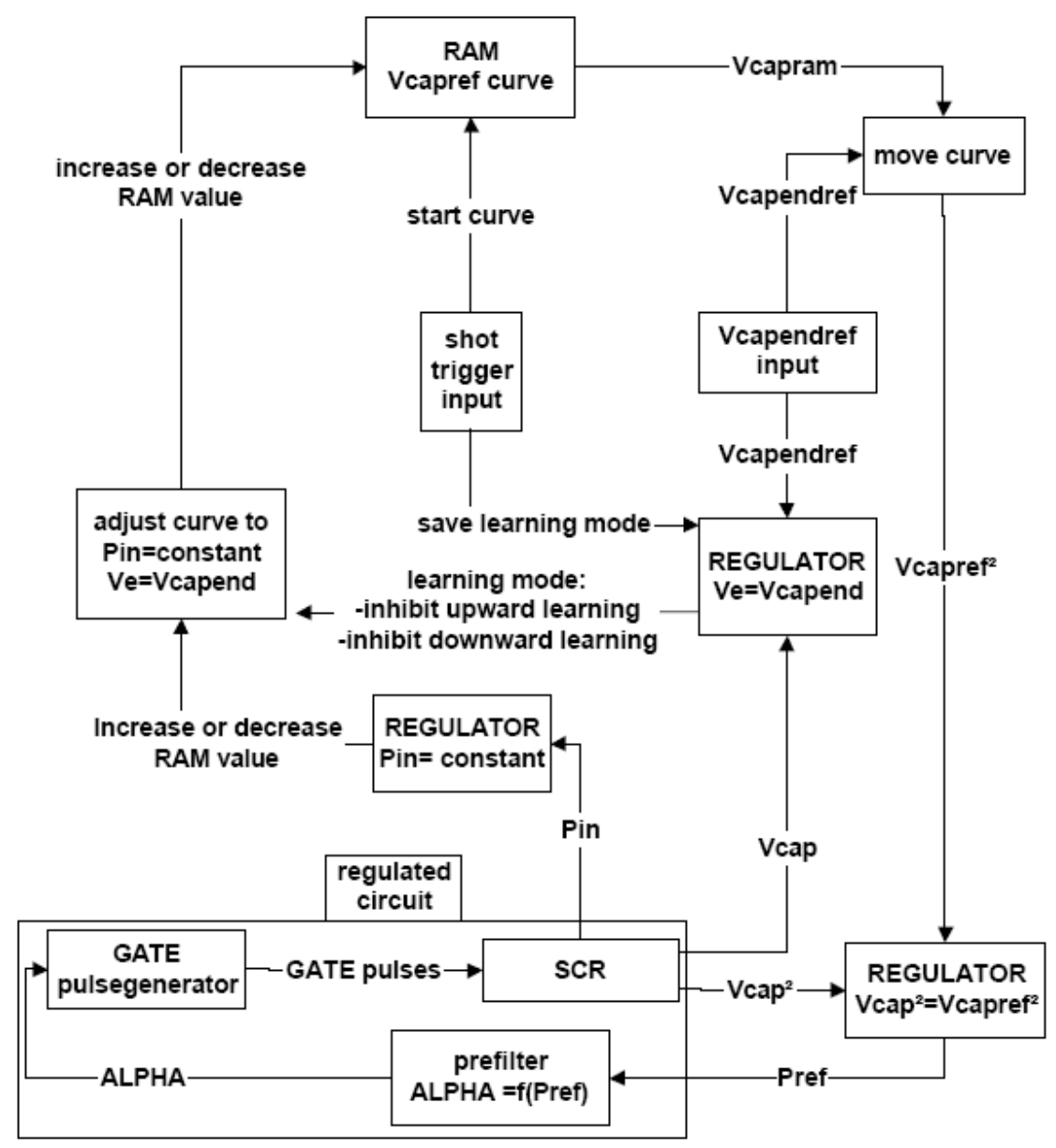

Figure 3.21: Block diagram of the digital self-learning regulation.

The reference value $\mathrm{UCloadref}^{2}$ and the linearized squared capacitor voltage $\mathrm{U}_{\mathrm{Cload}^{2}}$ are passed to a $\mathrm{P}(\mathrm{I})$ regulator. The signal $\mathrm{P}_{\text {ref }}$ is the signal steering the power consumption of the power supply (Figure 3.20). It is necessary that the actual input power $\mathrm{Pin}_{\mathrm{in}}$ is proportional to the steering signal $\mathrm{P}_{\text {ref }}\left(\mathrm{P}_{\text {in }} \sim \mathrm{P}_{\text {ref }}\right)$. In some power supplies a pre- filter has to be used to ensure that $\mathrm{P}_{\text {in }} \sim \mathrm{P}_{\text {ref }}$. Droops of the mains voltage UMAINs are the most disturbing error signals for the $\mathrm{P}(\mathrm{I})$ regulator. The pre filter in the proposed power supply for TESLA guarantees the independence of the input power $P_{\text {in }}$ from the mains voltage Umains $\left(\mathrm{P}_{\text {ref }} \sim \mathrm{P}_{\text {in }} \neq \mathrm{f}(\mathrm{UmaIns})\right)$.

The hardware of the regulation is based on a programmable ALTERA device. The device contains the complete regulation software, the pulse firing generation of the switched mode power supply and the interlocks for the power supply. The designed board is able to work in local mode. A VME interface allows communicating with a control computer to feed in precalculated curve forms for the modulator. By this the learning time is decreased. The regulation scheme is shown in Figure 3.21. 


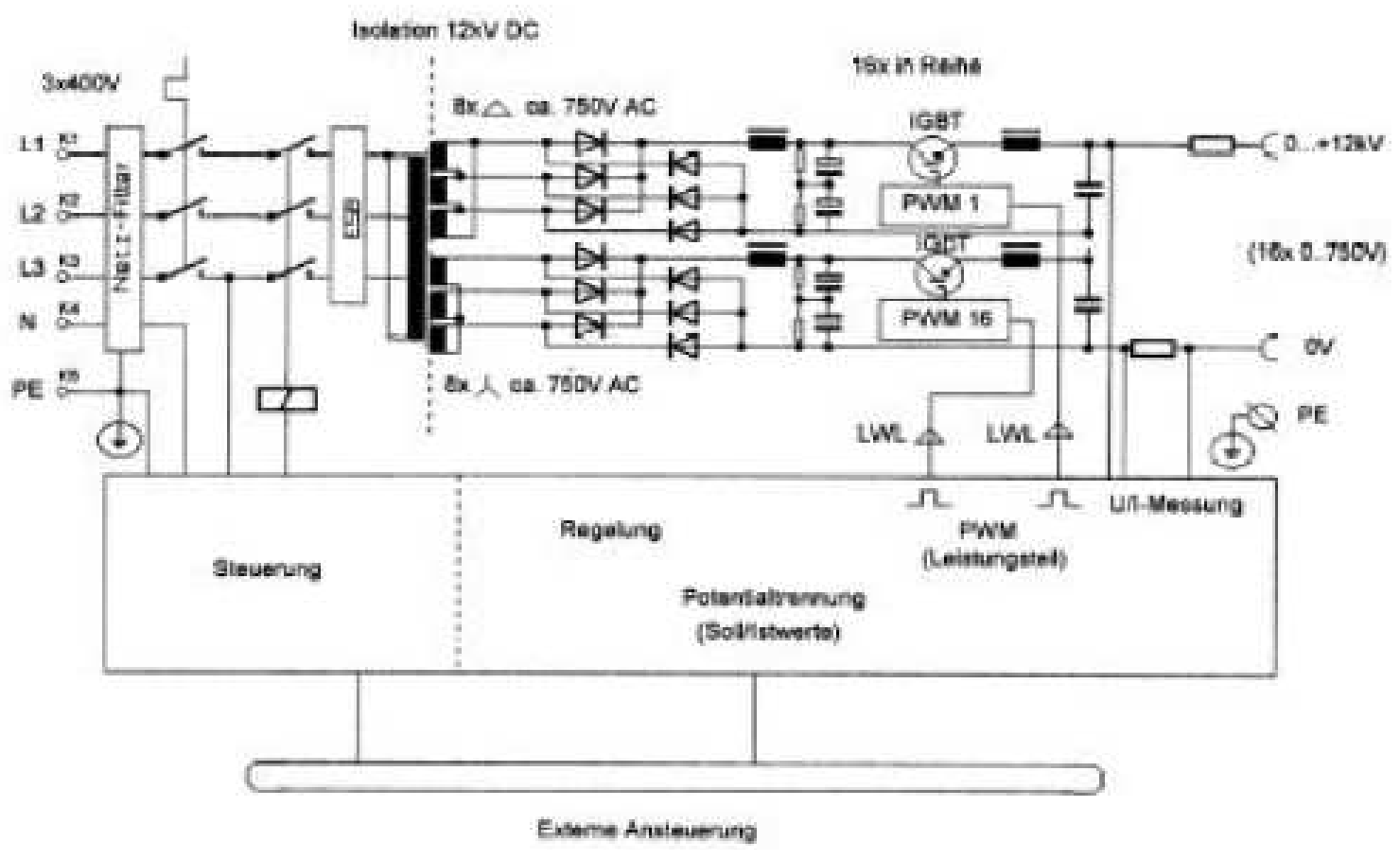

Figure 3.22: Power supplies with stacked buck converters.

\subsubsection{Series connection of buck converters (stacked unit)}

To achieve the high voltage level of the main capacitor bank it is possible to stack a group of low voltage power supplies. This topology is used for power supplies that manufactured in industry. The units are now under construction and will be used for some of the TTF modulators. The power supplies are buck converters each having a nominal voltage of app. $750 \mathrm{~V}$ each. 16 modules are stacked to deliver the required voltage of $12 \mathrm{kV}$. The transformer has a $400 \mathrm{~V}$ input and 16 outputs of which 8 outputs are in delta, the other 8 outputs are in star to get less mains harmonics. Figure 3.22 shows the principle diagram of such a power supply. The buck converters are working with a Pulse Width Modulation (PWM). The overlaid regulation for the voltage and the constant input power is accomplished on an external board that is developed and provided by DESY. The internal regulation of the power supply is a power regulation. The units will receive the power reference signal from the DESY regulation and transforms this into voltage and current values. 


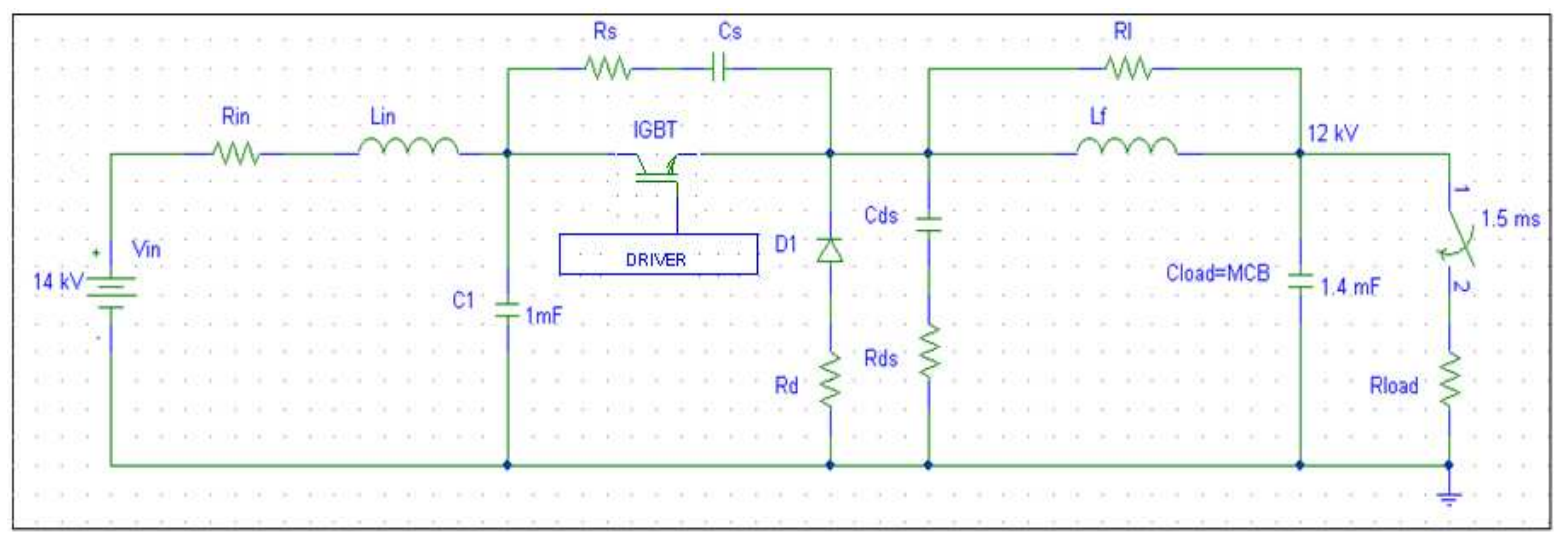

Figure 3.23: The overall layout of buck converter.

\subsection{The Buck converter solution}

The previous chapter has shown several modulator power supply design analysis. At this point the basic idea is to realize a modulator power supply project constituted by a high voltage buck converter (or step down) single unit. The device, that is supplied by an input stabilized high voltage of $14 \mathrm{kV}$, must charge the main capacitor bank (with a project value of $1.4 \mathrm{mF}$ ) of each single modulator with a maximum voltage of about $12 \mathrm{kV}$ as request by ILC reference design report. Initially will be shown and explained the schematic of device and in particular will be exposed the requirements of buck converter. After, a circuit simulation will be implemented to analyze the appropriateness of device functionality with the requirements.

\subsubsection{Buck converter schematic and design requirements}

The requirements of buck converter are mentioned as follows:

- Constant power functionality;

- An input voltage of $14 \mathrm{kV}$ and output voltage of $\sim 12 \mathrm{kV}$;

- The high voltage storage capacitor of the main capacitor bank charged in $100 \mathrm{~ms}$ from $10 \mathrm{kV}$ to $\sim 12 \mathrm{kV}$ considering $10 \mathrm{kV}$ as initial voltage value of main capacitor bank;

- The main capacitor bank discharged in $1.5 \mathrm{~ms}$ from $\sim 12 \mathrm{kV}$ to $10 \mathrm{kV}$;

- A switching frequency of $25 \mathrm{kHz}$ ( and a switching period of $40 \mu \mathrm{s}$ );

- A duty factor D of $0.75 \div 0.86$. 
The Figure 3.23 shows the circuital structure of the device that will be analyzed. The principal parts of buck converter and them functionalities are:

- An input LC filter that isolates power lines from harmonics generated during the main switch turn-on/off; the value of input inductance Lin is $10 \mu \mathrm{H}$ while the value of input capacitor $\mathrm{C}_{1}$ is $1 \mathrm{mF}$;

- A main switch characterized by an IGBT stacked switch working in hard switching. It produces a variable voltage proportional to duty factor;

- A free-wheeling diode $\mathrm{D}_{1}$ that allows path of inductor current during the mains switch turn-off;

- The main switch and diode RC snubber circuits that limit overvoltage on switches during them turn-off; the value of snubber resistance and capacitor chosen are $200 \Omega$ and $1 \eta \mathrm{F}$;

- A charging inductance $\mathrm{L}_{\mathrm{f}}$ that reduces the main capacitor bank charging current ripple to an appropriate value. The $\mathrm{L}_{\mathrm{f}}$ value can be calculated in the following way.

The main capacitor bank $\mathrm{C}_{\text {load }}$ must be charge in $100 \mathrm{~ms}$ from $10 \mathrm{kV}$ to $12 \mathrm{kV}$. Its charging current is

$$
I c=C_{\text {load }} * \frac{\Delta V_{c}}{\Delta t}=1.4 m F * \frac{2 k V}{100 m s} \approx 30 \mathrm{~A}
$$

Now considering the inductance charging current variation of $10 \%$, we can calculate the $\mathrm{L}_{\text {filter }}$ value as

$$
L=\frac{\Delta t * V_{L}}{\Delta I_{L}} \cong \frac{\frac{1}{2 f} * 2 k V}{3 A} \cong \frac{\frac{1}{50 k H z} * 2 k V}{3 A} \cong 13.5 \mathrm{mH}
$$

- The $\mathrm{R}_{\text {load }}$ represents the modulator resistance and in particular the value chosen is 7.6 $\Omega$.

\subsubsection{Circuit simulations and analysis}

The circuit simulation have been implemented using WinSpice3 (version 11/2004) a generalpurpose circuit simulation for non-linear DC, non-linear transient and linear AC analyses. It is based on Spice3 developed by Department of Electrical Engineering and Computer Sciences, University of California, Berkeley. We have performed a transient analysis to computes the transient output variables as a function of time over a user-specified time interval. 

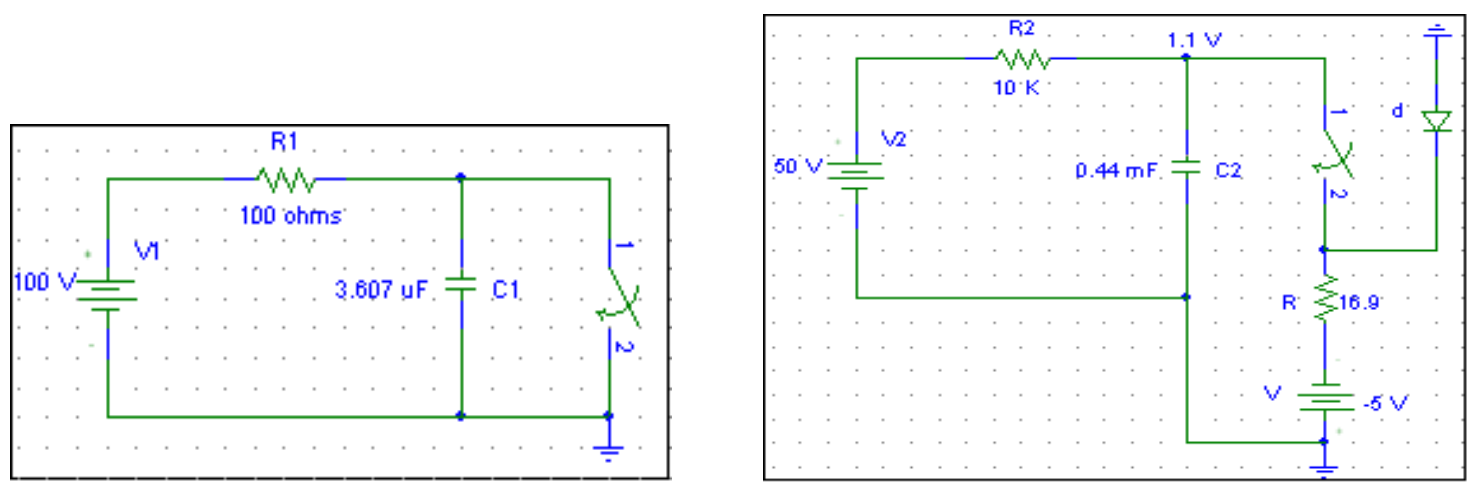

Figure 3.24: a) RC Circuit for carrier waveform; b) RC Circuit for modulating waveform.

The initial conditions are automatically determined by a DC analysis that determines the DC operating point of the circuit with inductors shorted and capacitors opened. The circuit to be analyzed is described to WinSpice 3 by a set of element lines, which define the circuit topology and elements values and a set of control lines, which define the model parameters and the run controls.

The main circuit elements and simulation parameters used are:

- Linear resistors, capacitors and inductors;

- Diode and voltage controlled switches;

- DC voltage sources and independent sources as pulse sources to switches control;

- A transient period of $1 \mu \mathrm{s} \div 220 \mathrm{~ms}$;

Also the use of an ideal element that is highly non-linear such as a switch can cause large discontinuities to occur in the circuit node voltage. A rapid change such as that associated with a switch changing state can cause numerical round-off or tolerance problems leading to erroneous results or time-step difficulties. So to improve this situation was used:

- A tolerance on transient errors of $10^{-1}$;

- A relative tolerance of $10^{-4}$.

\subsubsection{Main switch control for constant power}

The core of project is to generate the control waveform of the main switch to obtain the buck converter constant power functionality. The basic idea is to generate a square root waveform with a variable duty factor $\mathrm{D}$ variation in the range $0.75 \div 0.86$ to guarantee the charging voltage of capacitor from $10 \mathrm{kV}$ to about $12 \mathrm{kV}$. The square root can be obtained comparing two waveforms: a carrier waveform and a modulating waveform. 


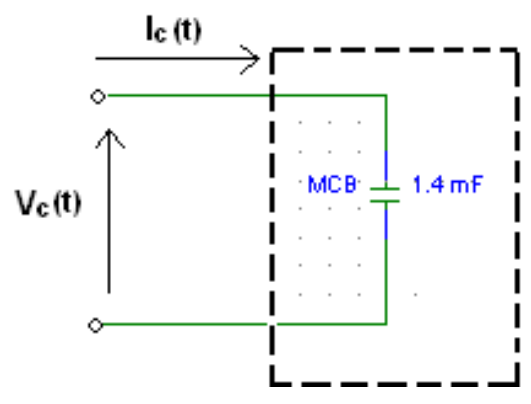

Figure 3.25: The main capacitor bank as black box.

As shown in Figure 3.24 a), a first RC circuit produces a sawtooth waveform or the carrier waveform that increases linearly each $40 \mu \mathrm{s}$ (switching period of stacked switch) from $0 \mathrm{~V}$ to $10 \mathrm{~V}$ and discharge immediately in about $20 \eta \mathrm{s}$. Now is important to understand what the modulating waveform to obtain constant power is.

Seeing the storage capacitor as a black box we can write the following system of equations

$$
\left\{\begin{array}{l}
P(t)=V_{c}(t) * I_{c}(t) \\
I_{c}(t)=C_{\text {laod }} \frac{d V_{c}}{d t}
\end{array}\right.
$$

To obtain constant power means

$$
P(t)=k \Rightarrow I_{c}(t)=\frac{k}{V_{c}(t)} \Rightarrow \frac{k}{V_{c}(t)}=C_{\text {load }} \frac{d V_{c}}{d t}
$$

and integrating the two members we obtain

$$
\int_{V_{0}}^{V} V_{c} d V=\frac{K}{C_{\text {load }}} \int_{t_{0}}^{t} d t
$$

where $V_{0}$ is the initial voltage of capacitor and $V$ its final voltage while $t_{0}$ is the initial time and $t$ is the final time of charge. Resolving the integral we have the expression of voltage waveform to charge the capacitor for constant power functionality as

$$
V_{c}(t)=\left[\frac{2 k}{C_{\text {load }}} t+\left(V_{0}\right)^{2}-\frac{2 K}{C_{\text {load }}} t_{0}\right]^{1 / 2}
$$




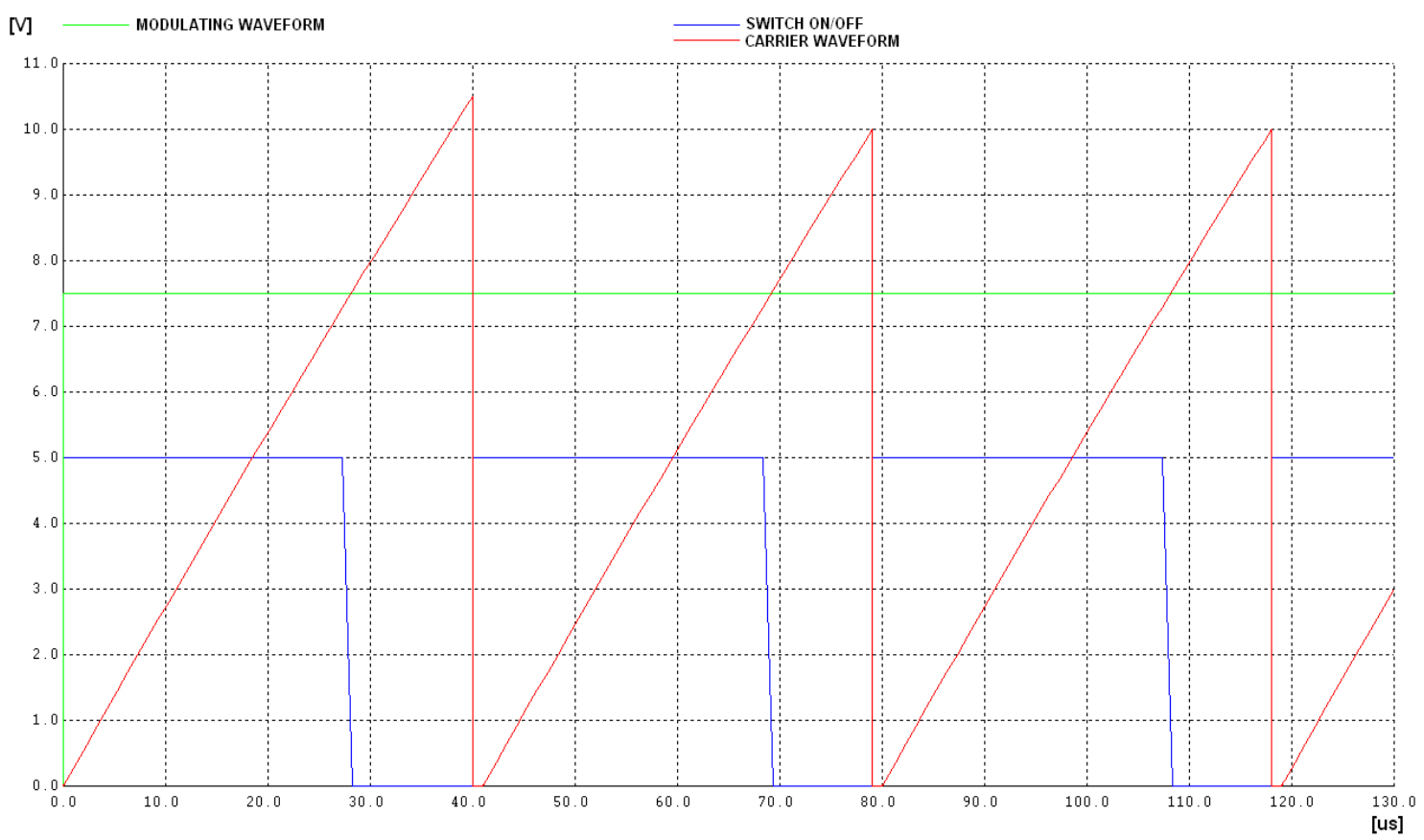

Figure 3.26: Control waveform of main switch for constant power.

Considering $\alpha=\frac{2 k}{C_{\text {load }}}$ and $\beta=\left(V_{0}\right)^{2}-\frac{2 k}{C_{\text {load }}} t_{0}$ the equation becomes

$$
V_{c}(t)=[\alpha * t+\beta]^{1 / 2}
$$

The relation (3.14) represents the analytical solution at our problem.

In our case $\alpha=177.1$ and $\beta=56.25$ and so

$$
V_{c}(t)=\sqrt{177.1 * t+56.25}
$$

is the modulating signal to have constant power.

At this point a second RC circuit (see Figure $3.24 \mathrm{~b}$ )) produces another saw tooth waveform that increases linearly each $100 \mathrm{~ms}$ from $0 \mathrm{~V}$ to $1.1 \mathrm{~V}$ and it discharges in $1.5 \mathrm{~ms}$. And so with the sqrt command WinSpice3, we have implemented the square root waveform of voltage capacitor described in the relation (3.15). 
[A]

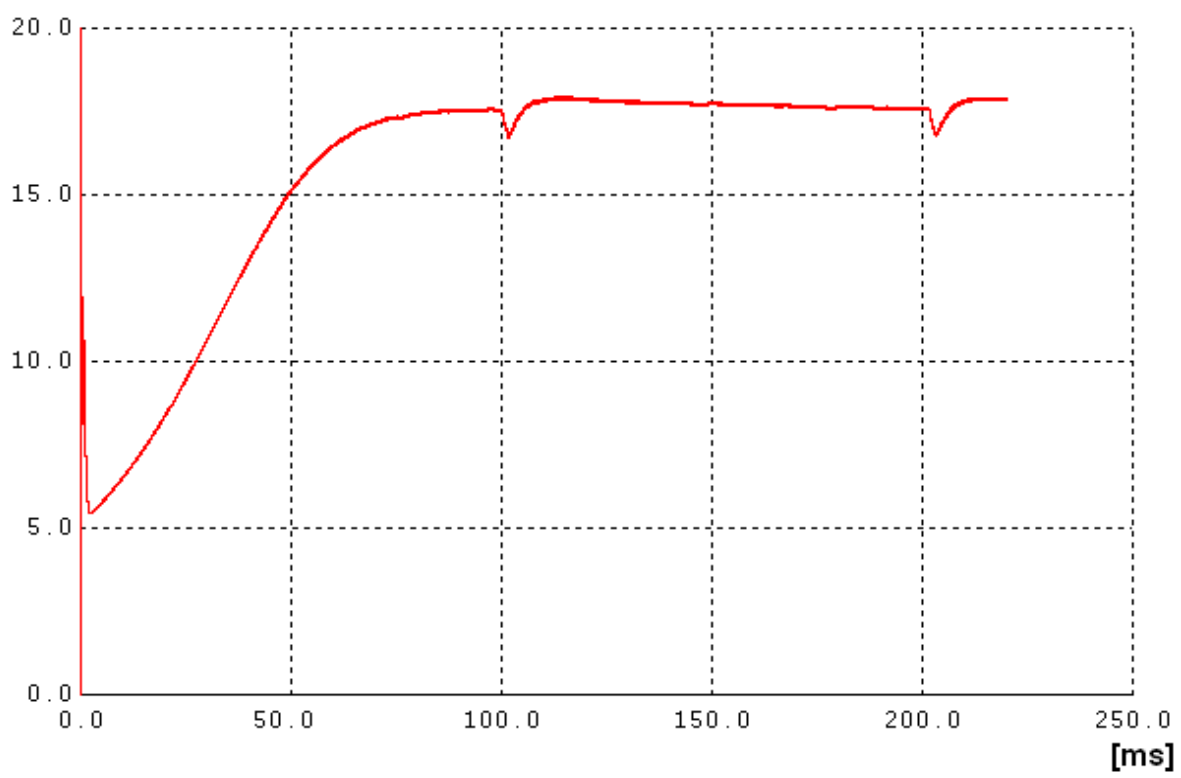

Figure 3.27: Buck converter input current.

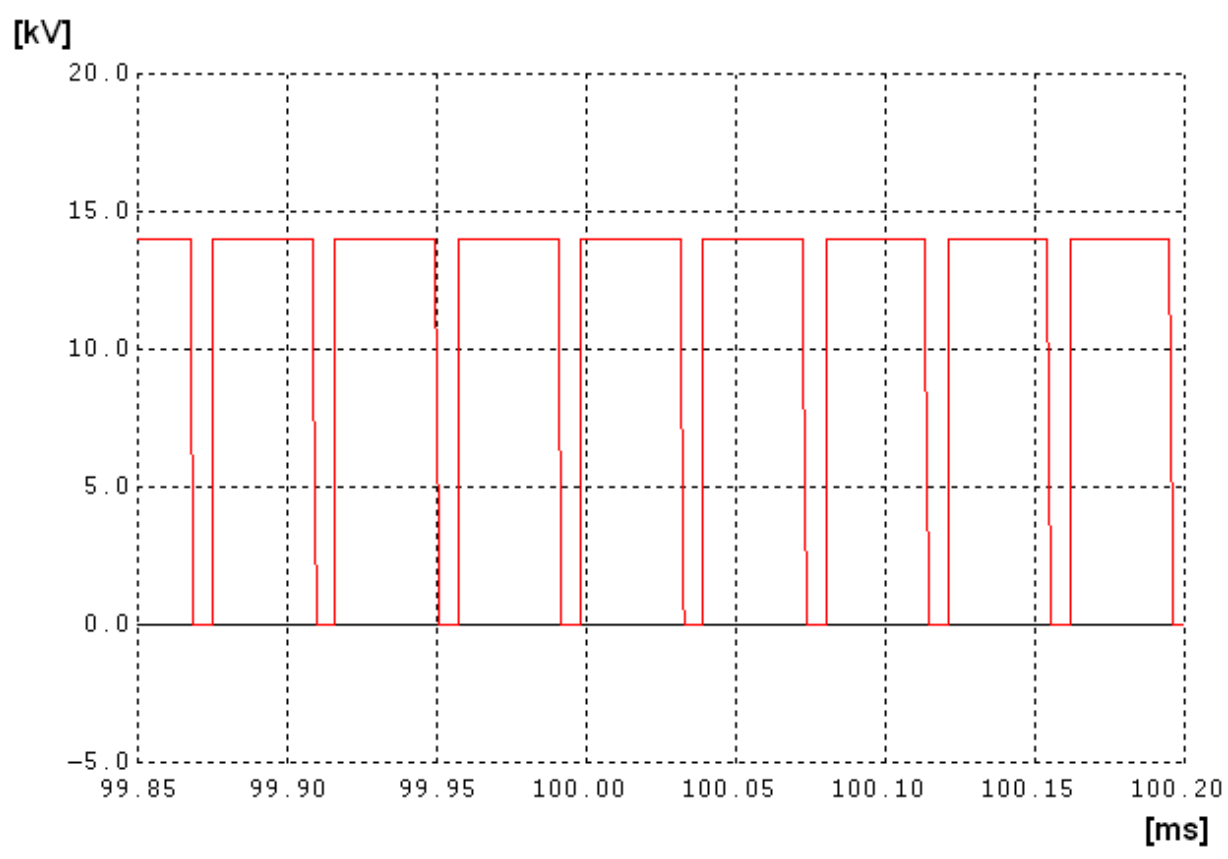

Figure 3.28: Main switch output voltage. 
[kV]

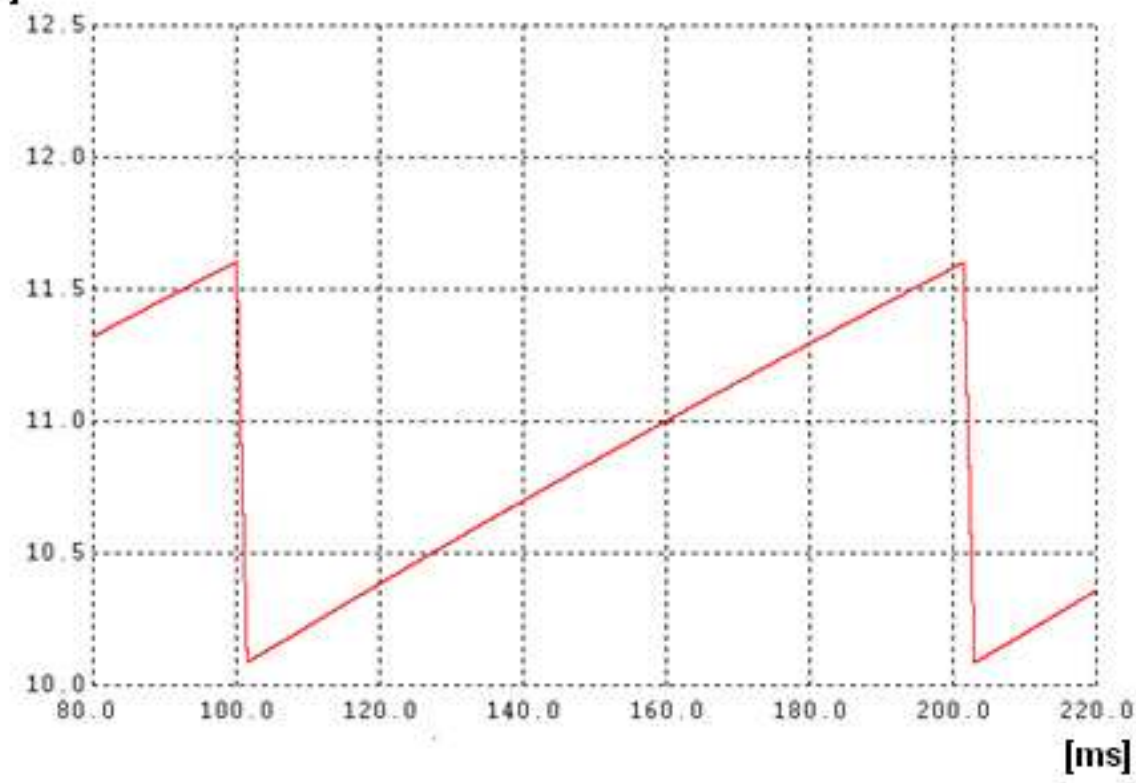

Figure 3.29: Main capacitor bank voltage.

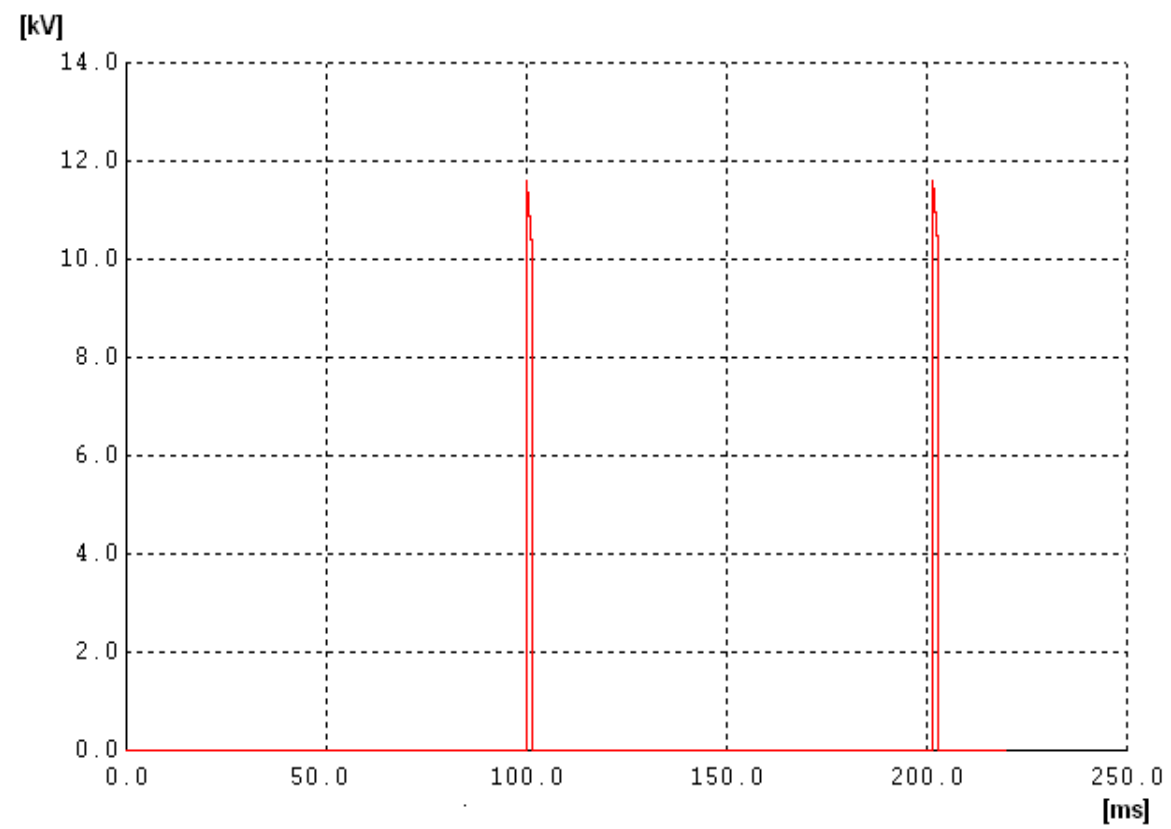

Figure 3.30: Modulator output pulses. 

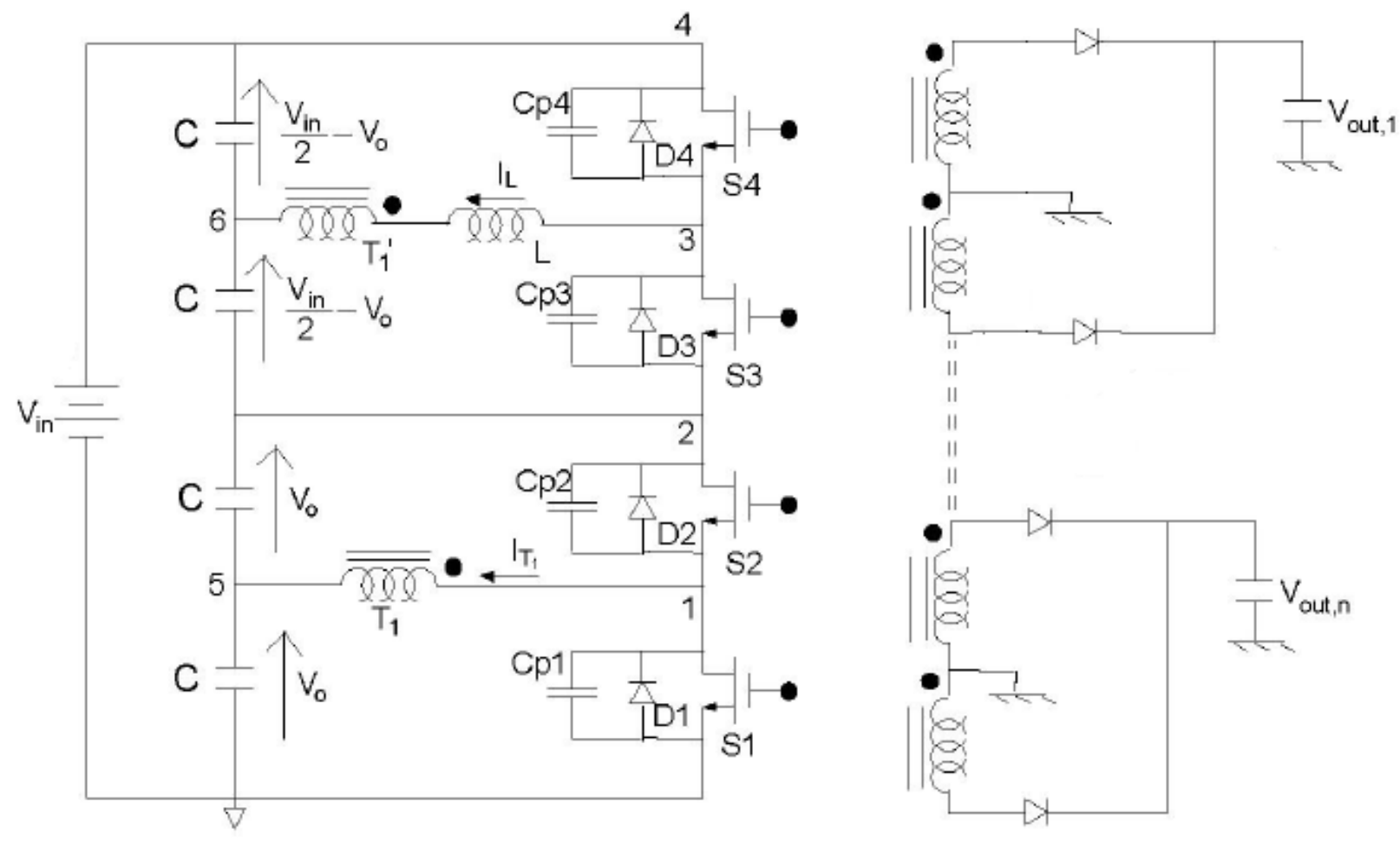

Figure 3.31: SILC Converter schematic.

\subsubsection{Buck converter waveforms}

In this paragraph we will analyzed the results of simulation and in particular will be analyzed the following waveforms: input current, main switch output voltage, main capacitor bank voltage and the modulator output voltage. The Figure 3.27 shows the modulator power supply current; it has a maximum value of 18 A. After an initial transitory, the current becomes constant in each period of $100 \mathrm{~ms}$. And so as the input voltage and the input current are constants in $100 \mathrm{~ms}$, the buck converter works in constant power. While as shown in Figure 3.28 the main switch output voltage is pretty good and this is proof that the switch works well. In particular it's a square root waveform with a duty factor in the range $0.75 \div$ 0.86 , with zero initial voltage and a final value of $14 \mathrm{kV}$. The Figure 3.29 represents the modulator main cap bank voltage. The voltage increases in $100 \mathrm{~ms}$ from $\sim 10 \mathrm{kV}$ to $11.6 \mathrm{kV}$ and it discharges in $1.5 \mathrm{~ms}$. Moreover the waveform follows the reference or modulating signal to obtain constant power. In the end the Figure 3.30 illustrates the modulator output voltage characterized by pulses with a period of time of $1.5 \mathrm{~ms}$, a maximum voltage value of about $12 \mathrm{kV}$ and a pulse repetition rate of $10 \mathrm{~Hz}$. 
Klystron Modulator Power Supplies

\subsection{The Switch In Line Converter}

Innovation in the field of switched power supplies and DC/DC converters has recently acquired a new impulse by the phase-shift (PS) control. Inherent advantages of this technique, in term of constant operating frequency, soft switching and dynamic simplicity, emerge from proposal of new topologies suited for applications in different ranges of power. This chapter will explain an high power DC/DC phase-shifted converter: the "Switch In Line Converter" (SILC) $[13,14,15,16]$, characterized by disposition in line of IGBTs and good soft switching performance.

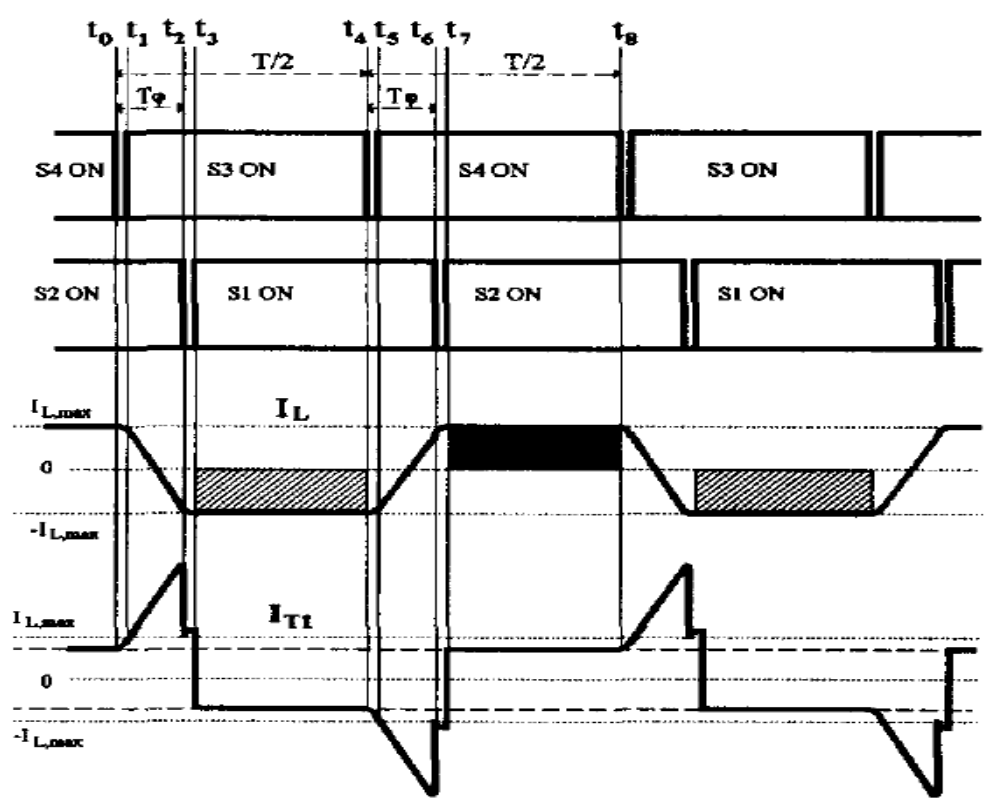

Figure 3.32: SILC switching commands and current waveforms.

\subsubsection{Operation Principle of the SILC Converter}

In the Figure 3.31, the proposed circuit topology of the SILC Converter is shown. It consists in the series connection of two switched half bridges (lower HB and upper HB). The inductor current $I_{L}$ is controlled by the phase-lead shift of the commands to S3, S4 relative to the commands to S1, S2. In the Figure 3.32, the ideal switching sequence and the current waveforms in the windings $\mathrm{T} 1$ ' and into $\mathrm{T} 1$ are represented. Reference is made to the typical bias condition $V_{i n}=4 V_{O}$, which means that there is the same steady-state voltage on each HB. The initial status, at $t=t_{0}{ }^{-}$, of the switches is: S1 OFF, S2 ON, S3 OFF and S4 ON. 


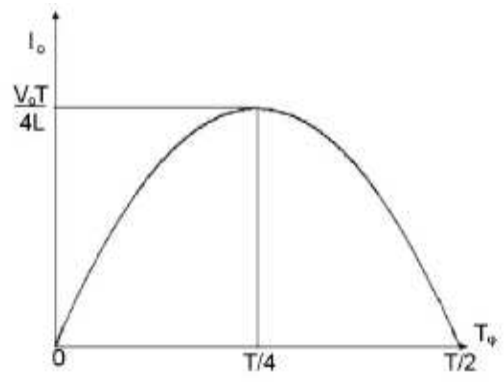

Figure 3.33: Dependence $f$ the output current on the phase shift delay.

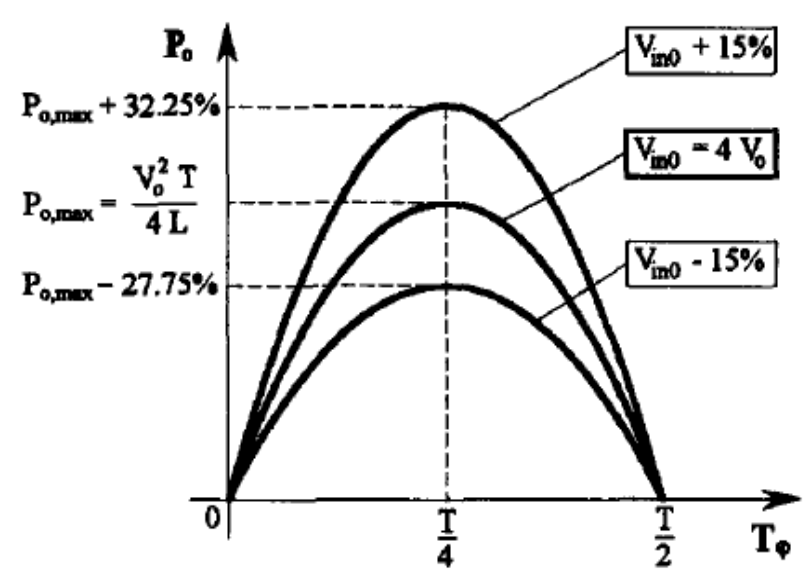

Figure 3.34: Phase-shift control characteristic.

At $t=t_{0}{ }^{+}$, S4 turns OFF: the current $\mathrm{I}_{\mathrm{L}}$ flows through $\mathrm{C}_{\mathrm{p} 4}$ and $\mathrm{C}_{\mathrm{p} 3}$ during the soft switching interval until D3 clamps; S3 turn ON at $t=t_{l}$. Then, since the voltage across $\mathrm{L}$ is $-2 \mathrm{~V}_{\mathrm{O}}, \mathrm{I}_{\mathrm{L}}$ decreases and reverses its polarity until it reaches the value $-\mathrm{I}_{\mathrm{L}, \max }$ at $t=t_{2}{ }^{-}$, when $\mathrm{S} 2$ turns OFF and the output rectifiers too. Current balance in the transformer implies that $I_{T 1}+I=I_{T 1}$ - $I_{L, \max }=0$, hence $I_{T l}=I_{L, \max }$. Another resonant voltage clamped transition, identical to the previous one, occurs at node 1 . Switch $\mathrm{S} 1$ turns $\mathrm{ON}$ at $t=t_{3}{ }^{-}$after clamping of D1. From $\mathrm{t}_{3}$ to $\mathrm{t}_{4}$ the current $\mathrm{I}_{\mathrm{L}}$ remains constant; in this interval the Kirchhoff law implies that $I_{T I}+I_{L}=I_{0}$, where $\mathrm{I}_{0}$ is the output current reflected to the primary side of the transformer. When S3 switches OFF $\left(t=t_{4}{ }^{-}\right)$, the second half period begins and it exactly mirrors the first one. The period ends at $t=t_{8}=t_{0}+T$. Power transfer to the load occurs during the time slots $t_{3}-t_{4}$ and $t_{7}-t_{8}$ only and it is $\mathrm{P}=\mathrm{V}_{\text {in }} \mathrm{Q} / \mathrm{T}$, where $\mathrm{Q}$ is the charge in the shaded area of Figure 6.0.To simplify, let's make reference to a single equivalent output voltage $\mathrm{V}_{\text {OUT }}=\mathrm{V}_{\mathrm{O}}$ corresponding to a unity winding ratio supplying an output current $\mathrm{I}_{\mathrm{OUT}}=\mathrm{I}_{\mathrm{O}}$, so that $\mathrm{P}=\mathrm{V}_{\mathrm{O}} * \mathrm{I}_{\mathrm{O}}$. Being and

$$
Q-2 i_{L, \max }\left(\frac{T}{2}-T_{\varphi}\right)-\frac{V_{i n} T_{\varphi}}{2 T}\left(\frac{T}{2}-T_{\varphi}\right)
$$


assuming zero ripple in the capacitor, that is $c \rightarrow \infty$, the output current referred to $\mathrm{V}_{\mathrm{O}}$ is given by :

$$
I_{O}=\frac{V_{i n}^{2} T_{\varphi}}{4 L T v_{O}}\left(\frac{T}{2}-T_{\varphi}\right)=\frac{V_{\ln } T_{\varphi}}{L T}\left(\frac{T}{2}-T_{\varphi}\right)
$$

The parabolic dependence of $\mathrm{I}_{\mathrm{O}}$ on $T_{\psi z}$ is shown in the Figure 3.33, where the operative control range emerges to be $0<T_{n}<\mathrm{T} / 4$. The advantages of the SILC topology are:

- the input voltage partition due the series connection of the half bridges reduces the stress of the power switches and the voltage transient amplitude at the switching resonance;

- the converter acts like a controlled current generator ;

- increased ZVS range and efficiency;

- "integrated-magnetic" feasibility, as L can be obtained by the leakage inductance of T1';

- non isolated feedback;

- $\quad$ suitability for multiple output in series or in parallel;

- nearly symmetrical soft switching;

- inherent power limitation and short circuit protection;

- natural output voltage clamping;

- high failure tolerance because of the in-line disposition of the switches;

- single pole dynamics.

\subsubsection{The $30 \mathrm{~kW}$ SILC Project}

The previous chapter has shown the detailed SILC operation. At this point the basic idea is to realize a high DC/DC switching power supply constituted by a Switch In Line Converter of $30 \mathrm{~kW}$. The device, that's supplied by an input stabilized low voltage of $560 \mathrm{~V}$, must charge a main capacitor bank with a maximum voltage of $1.4 \mathrm{kV}$. Initially will be exposed the requirements of SILC. After, a circuit simulation will be implemented to analyze the appropriateness of device functionality with the requirements. 


\subsubsection{SILC Design Requirements and Project}

The requirements of SILC converter are mentioned as follows:

- Constant power functionality;

- An input voltage of $560 \mathrm{~V}$ and an output voltage of $1.4 \mathrm{kV}$;

- The main capacitor bank charged in $100 \mathrm{~ms}$ up till $1.4 \mathrm{kV}$;

- The main capacitor bank discharged in $2 \mathrm{~ms}$ from $1.4 \mathrm{kV}$ to $1.3 \mathrm{kV}$ considering a droop voltage of $100 \mathrm{~V}$;

- A load current of $1200 \mathrm{~A}$;

- A switching frequency of $15 \mathrm{kHz}$ ( and a switching period of $66 \mu \mathrm{s}$ );

- A duty cycle $\mathrm{D}$ of $\sim 50 \%$.

According with these requirements, the values of components was chosen as follows:

- the final level voltage of main capacitor bank is $1.4 \mathrm{kV}$. Also, the main capacitor bank supplies load with $1200 \mathrm{~A}$ in $2 \mathrm{~ms}$. Assuming a droop voltage of the main capacitor bank equals to $100 \mathrm{~V}$ the value of the capacitor is :

$$
C_{\text {load }}=\frac{I_{\sigma} * \Delta T}{\Delta V}=\frac{1200 \mathrm{~A} * 2 \mathrm{mg}}{100 \mathrm{~V}}=24 \mathrm{mF}
$$

- the value of load resistance is :

$$
R_{\text {load }}=\frac{1400 \mathrm{~V}}{1200 \mathrm{~A}}=1.16 \Omega
$$

- assuming that at the end of transient time the input capacitors are charged to a level voltage of $140 \mathrm{~V}$ the transformer has a turns ratio of 1:10. Besides, being the charging current of the main capacitor bank is about $\sim 25 \mathrm{~A}$, the primary current of the transformer is about $\sim 250 \mathrm{~A}$. At this point, the magnetizing inductance of transformer can be determined by the following equation :

$$
L_{m}=\frac{V_{L} * \Delta T}{\Delta I}=\frac{560 \mathrm{~V} * 33_{\mu s}}{50 \mathrm{~A}} \approx 400 \mu \mathrm{H}
$$

considering $\Delta T=T / 2$ and $\Delta I \sim 20 \% I_{\text {pmimary }}$.

- the value of input capacitors is $50 \mathrm{mF}$ and of inductor $\mathrm{L}$ is $5 \mu \mathrm{H}$. 


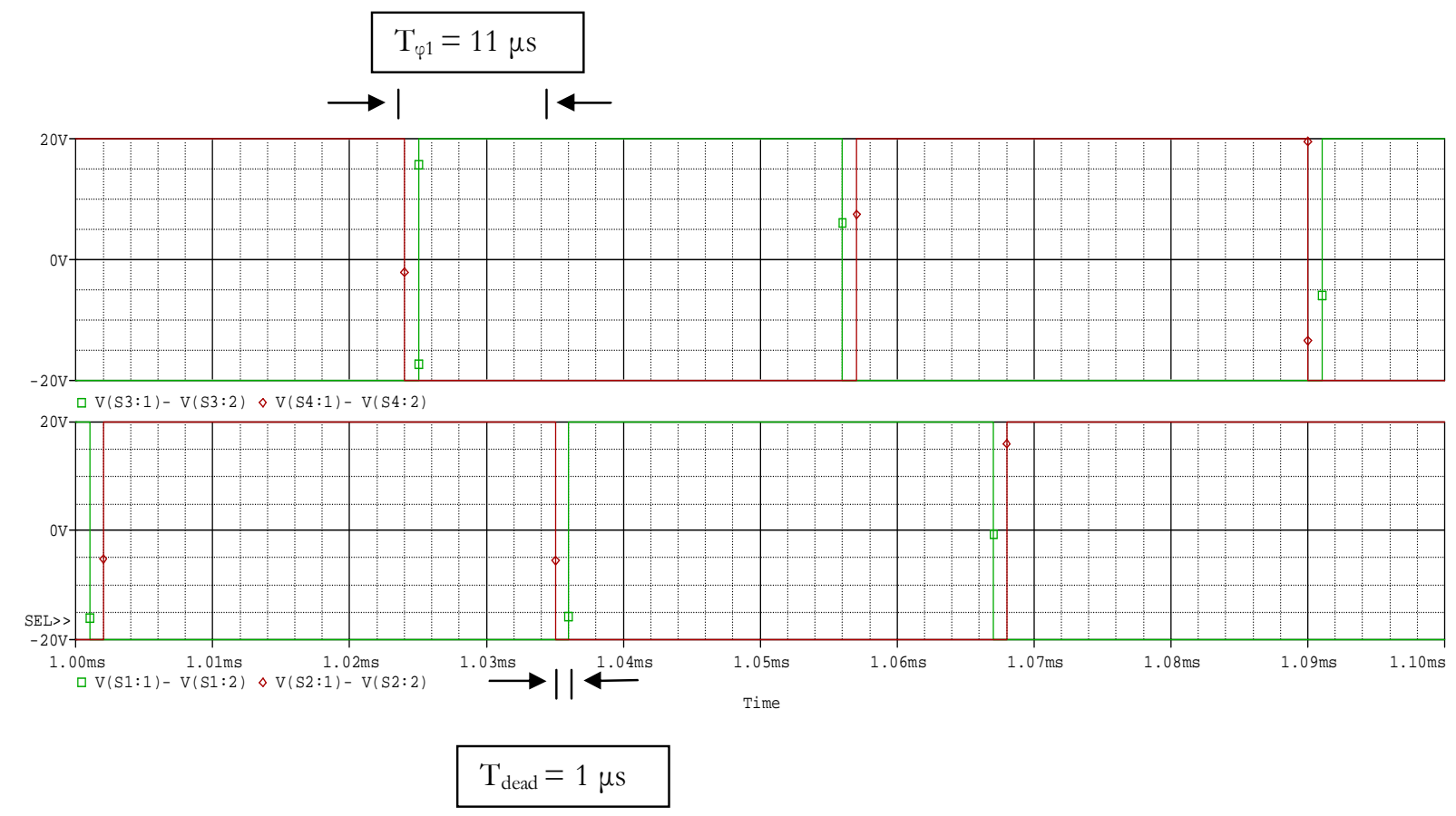

Figure 3.35: Switches control signals in the range time $0<\mathrm{t} \leq 100 \mathrm{~ms}$.

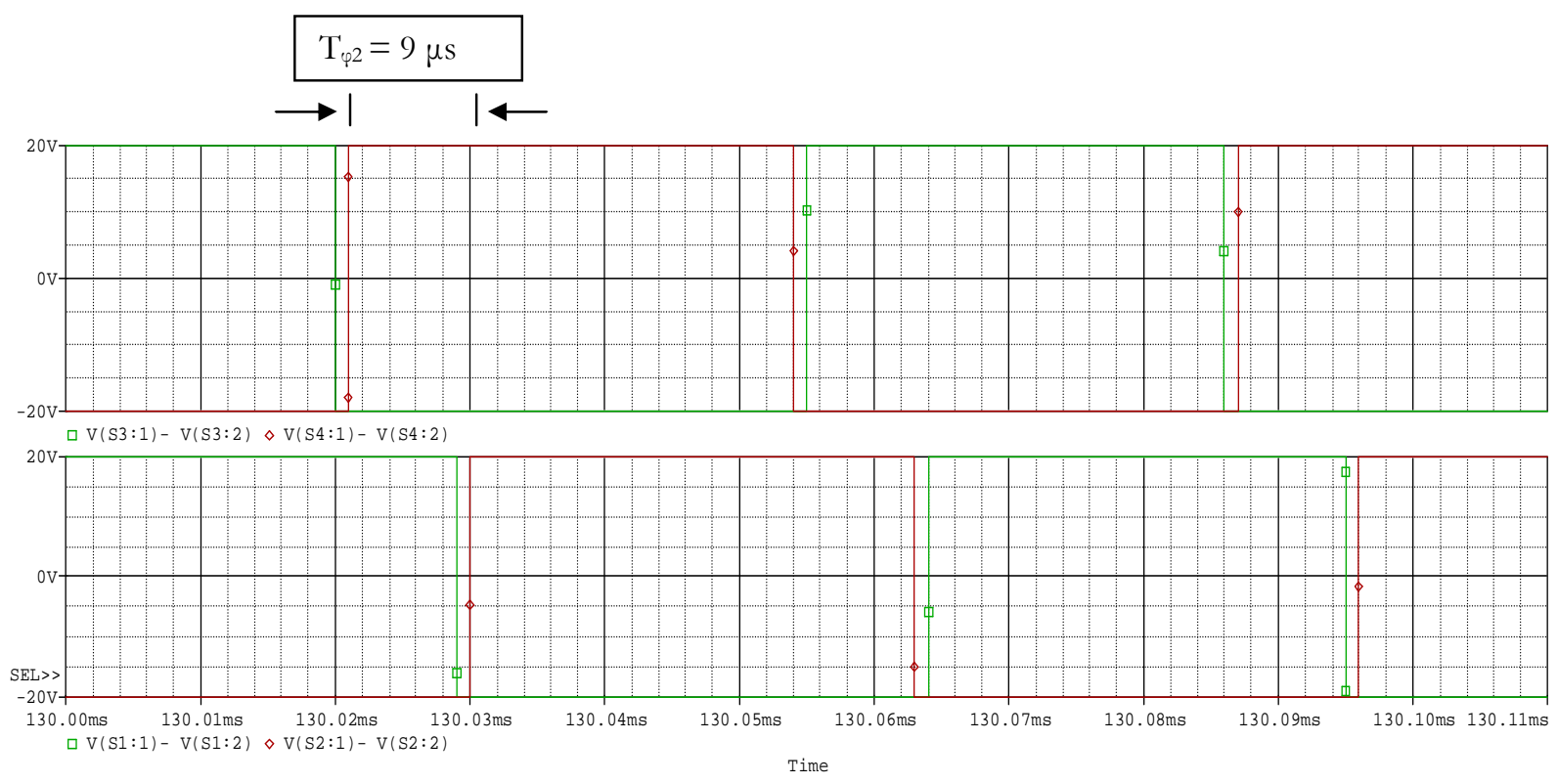

Figure 3.36: Switches control signals after $100 \mathrm{~ms}$. 
Klystron Modulator Power Supplies

\subsubsection{Circuit Simulations and Analysis}

The circuit simulations have been implemented using the Schematic session of OrCad 9.2 a general-purpose circuit simulation for non-linear DC, non-linear transient and linear AC analyses. We have performed a transient analysis to computes the transient output variables as a function of time over a user-specified time interval. The initial conditions are automatically determined by a DC analysis that determines the DC operating point of the circuit with inductors shorted and capacitors opened. The main circuit elements and simulation parameters used are:

- Linear resistors, capacitors and inductors;

- Power diode and power switches (IGBT);

- A step-up transformer with a coupling factor $\mathrm{k}=0.999$;

- DC voltage sources and independent sources as pulse sources to switches control;

- A transient period of $3 \mathrm{~s}$;

Also the use of an ideal element that is highly non-linear such as a switch can cause large discontinuities to occur in the circuit node voltage. A rapid change such as that associated with a switch changing state can cause numerical round-off or tolerance problems leading to erroneous results or time-step difficulties. So to improve this situation was used:

- A tolerance on transient errors of $10^{-1}$;

- A relative tolerance of $10^{-4}$.

\subsubsection{Control Signals of Switches}

According to the relation (3.17), the control of transfer power with the SILC topology is obtained with the control of the phase-lead shift of the commands to S3, S4 relative to the commands to $S 1, S 2$. In particular the maximum transfer power is achieved when $T \varphi=T / 4$ as well shown in the Figure 3.34. In the project was assumed a topology of control that consists to lead the switches commutation with a high value of $\mathrm{T}_{\varphi}$ until $100 \mathrm{~ms}$ and after the switches are controlled with a less $T_{\varphi}$ so that it is possible to control that the main capacitor bank is charged at level of voltage required. Accurately the values of $\mathrm{T}_{\varphi}$ chosen are:

- $\quad \mathrm{T}_{\varphi 1}=\mathrm{T} / 6=11 \mu \mathrm{s}(0<\mathrm{t} \leq 100 \mathrm{~ms})$

- $\quad \mathrm{T}_{\varphi 2} \sim \mathrm{T} / 7=9 \mu \mathrm{s} \quad($ after $100 \mathrm{~ms})$.

The Figures 3.35 and 3.36 show the control waveforms for switches. 


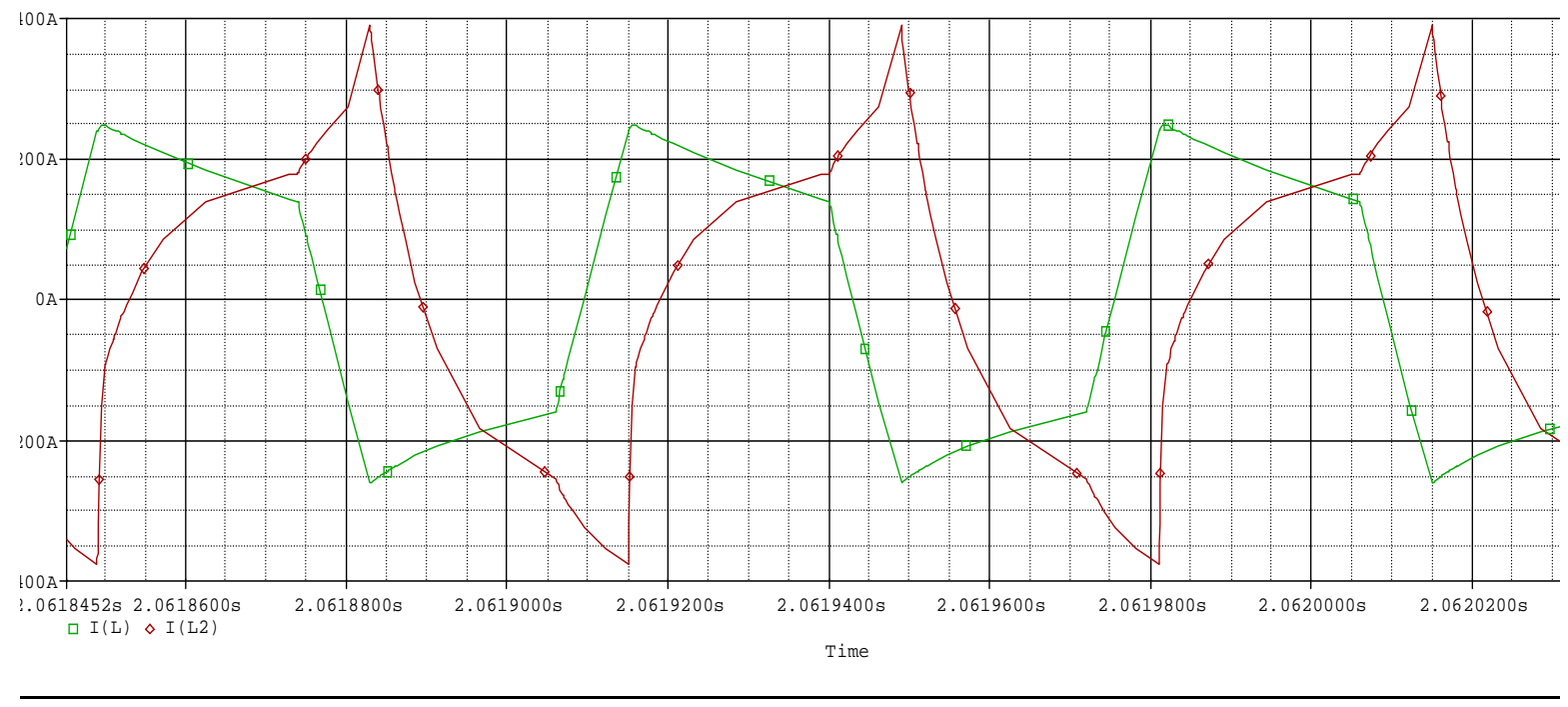

Figure 3.37: Transformer primary current windings T1' and T1.

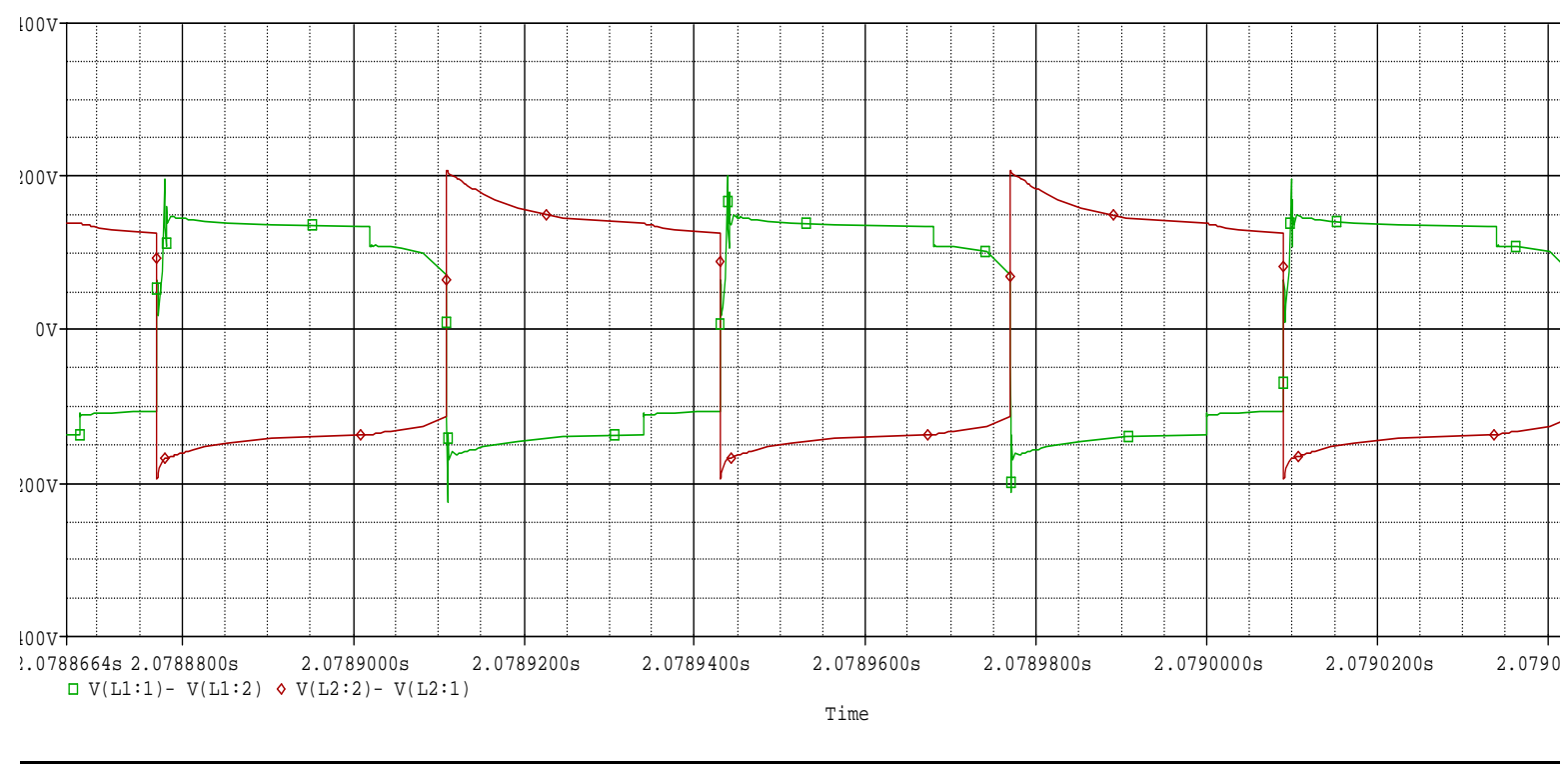

Figure 3.38: Transformer primary voltage windings $\mathrm{T} 1$ ' and $\mathrm{T} 1$. 


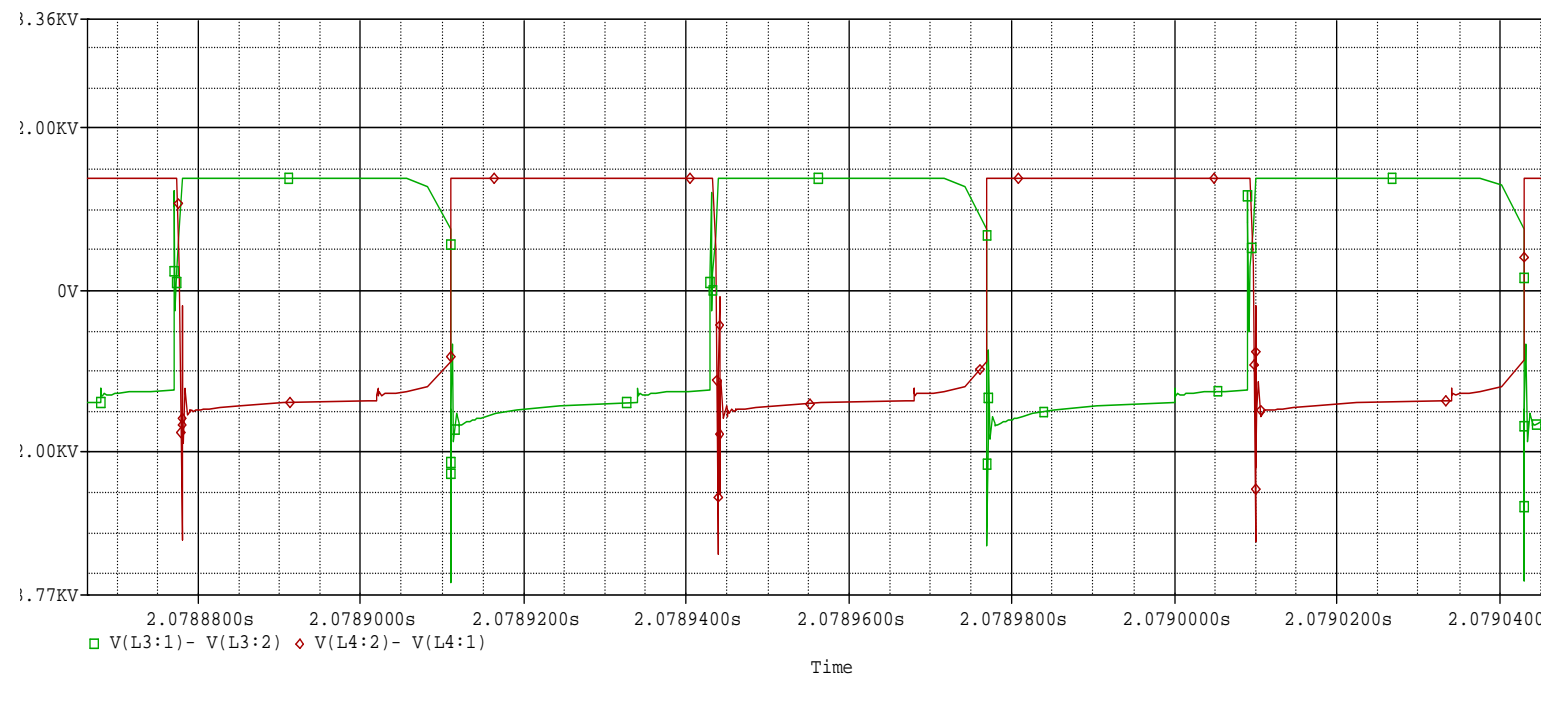

Figure 3.39: Voltage of the transformers secondary windings $\mathrm{T} 1$ ' and $\mathrm{T} 1$ in the configuration with center-tapped transformer.

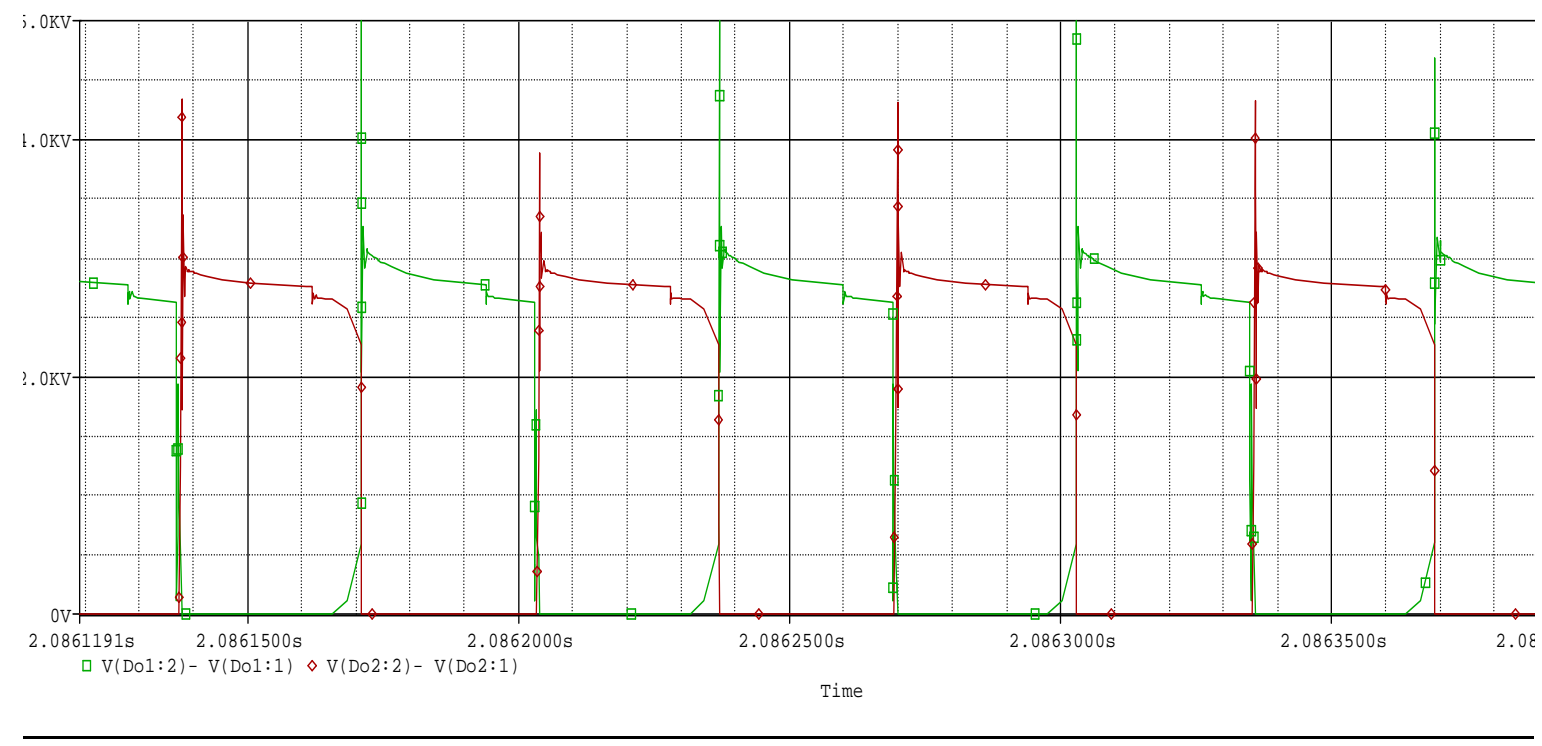

Figure 3.40: Voltage on the rectifier diodes in the configuration with center-tapped transformer. 


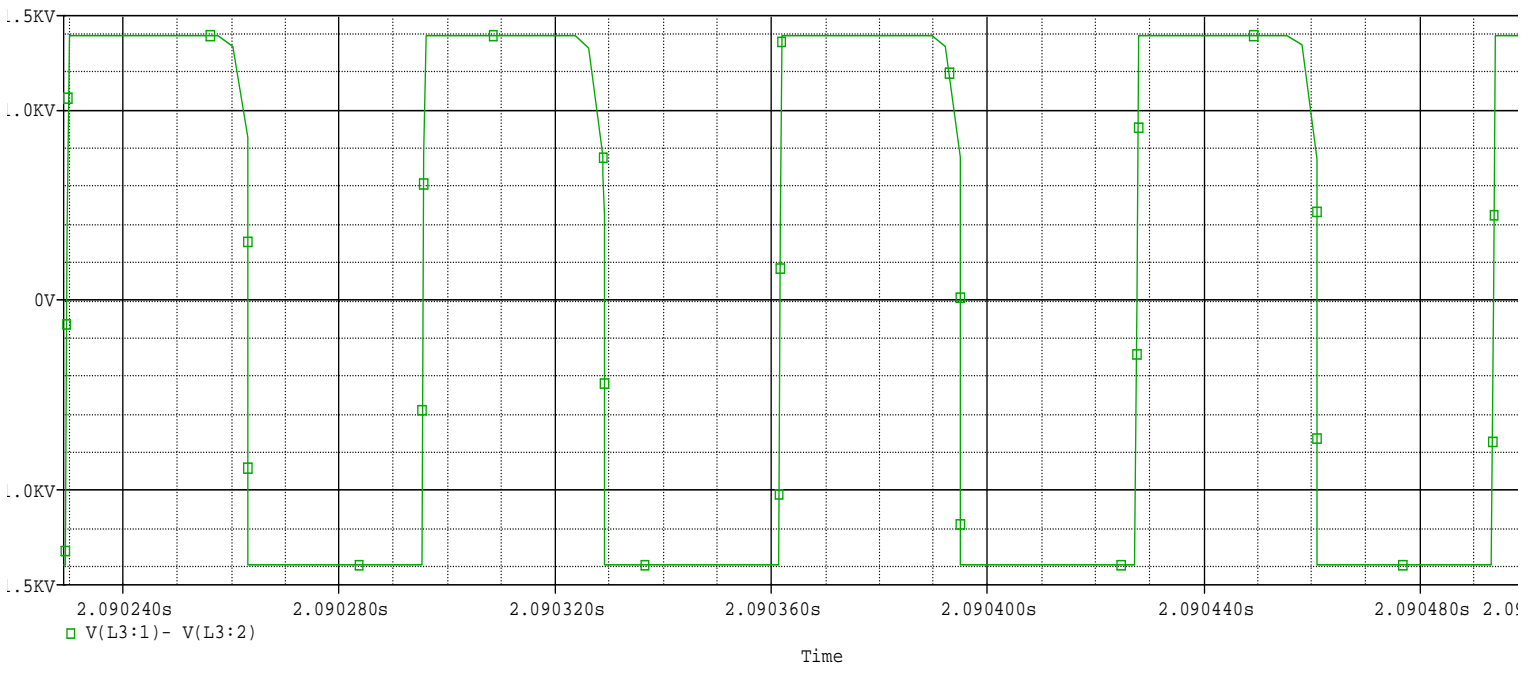

Figure 3.41: Voltage of the transformers secondary windings relatively at Graetz bridge configuration.

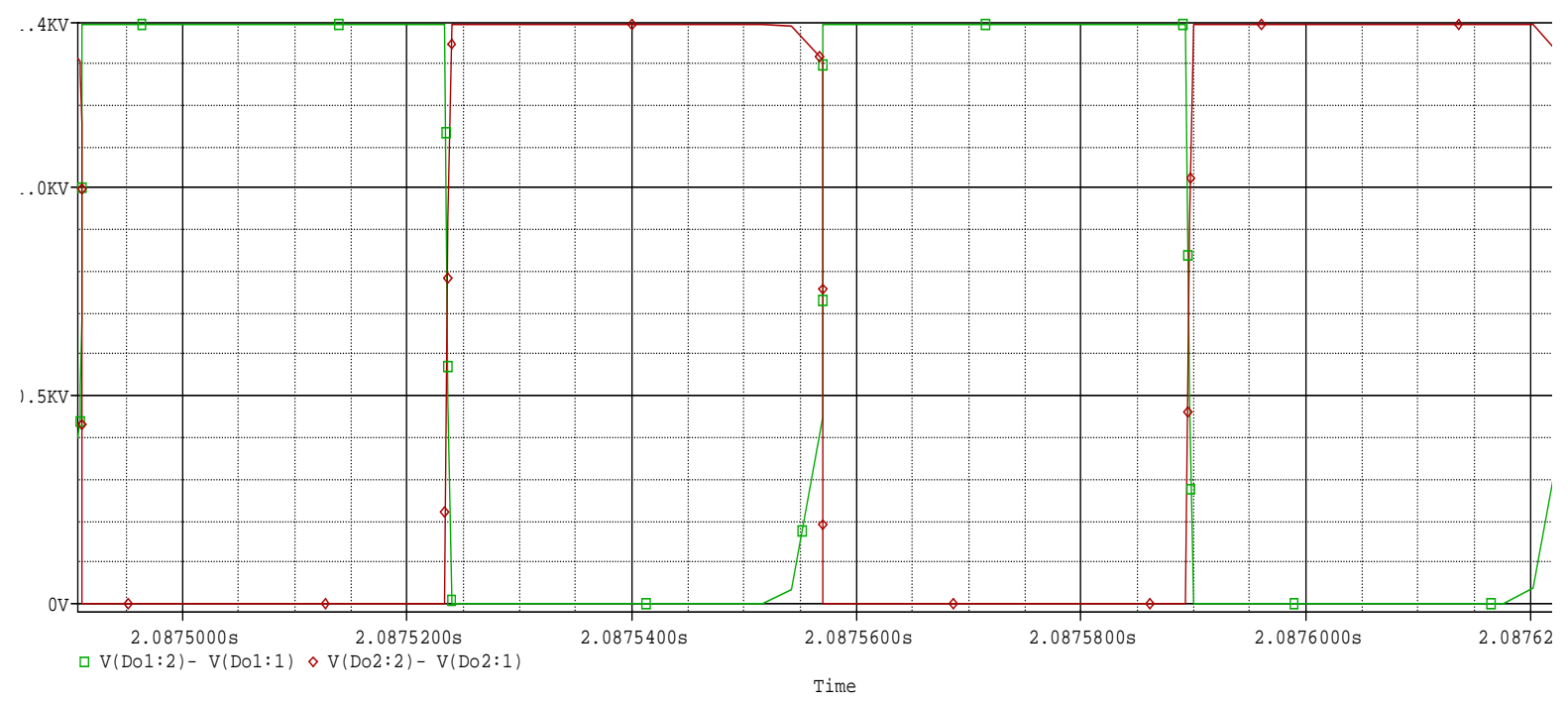

Figure 3.42: Voltage on the rectifier diodes in the configuration with Graetz bridge. 


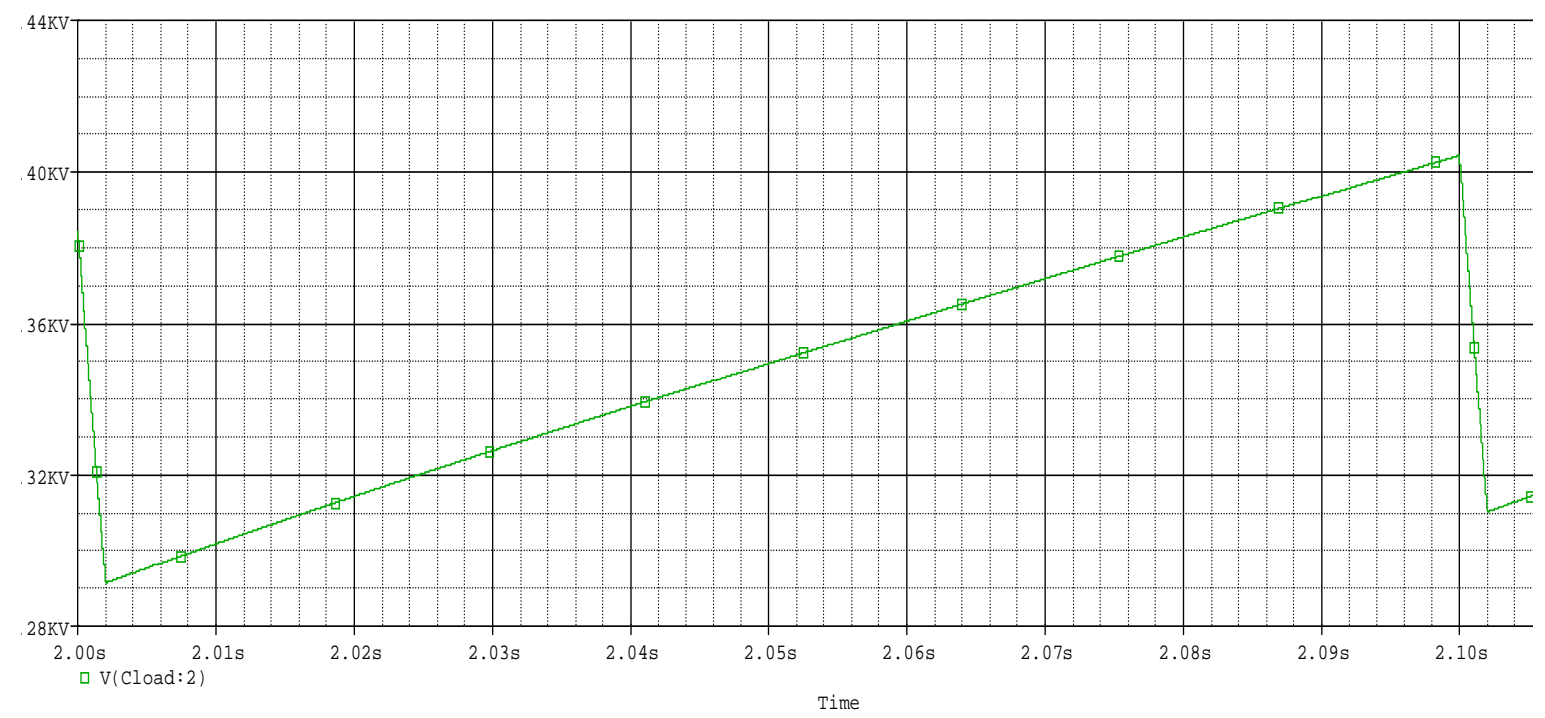

Figure 3.43: Main capacitor bank voltage.

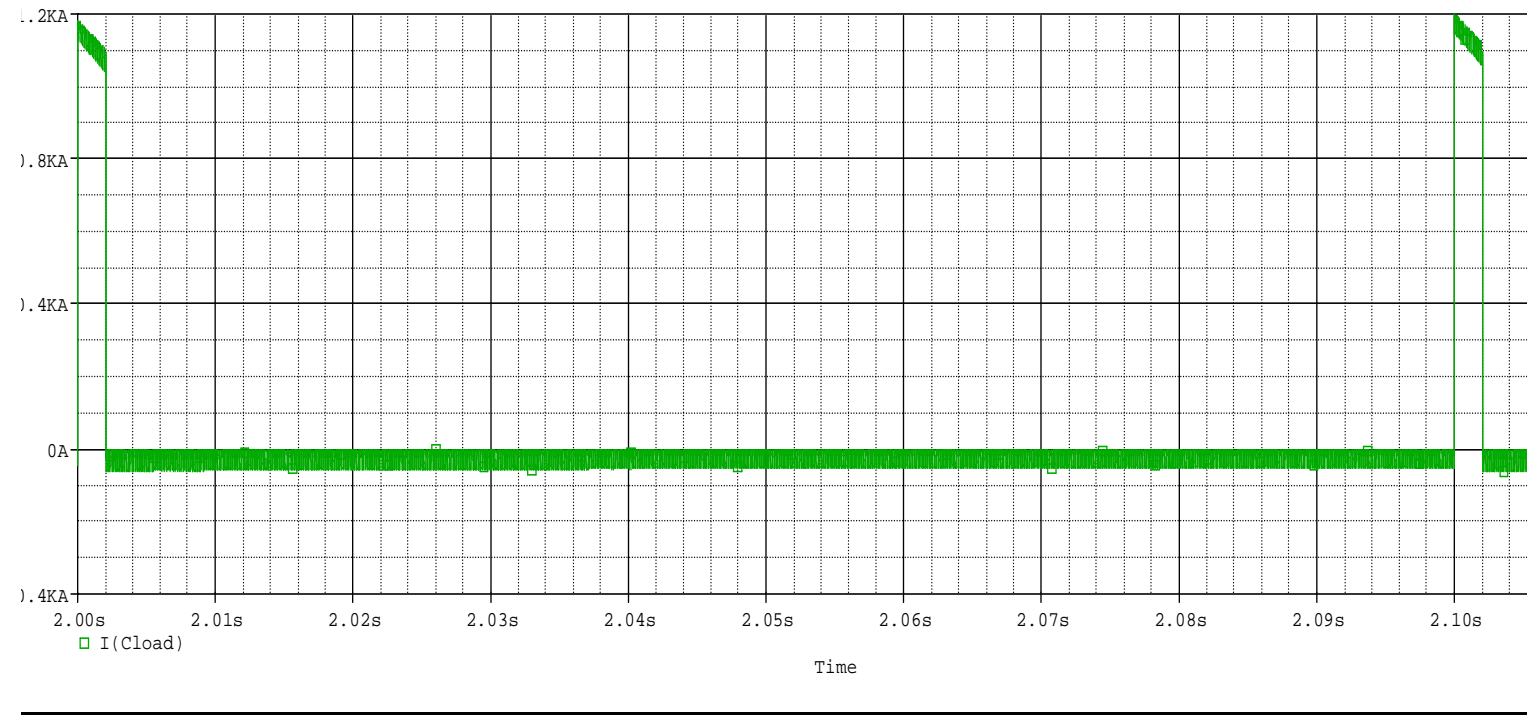

Figure 3.44: Charging and discharging current of the main capacitor bank. 
Klystron Modulator Power Supplies

\subsubsection{SILC Project Waveforms}

This paragraph analyzes the SILC waveform obtained with circuit simulations. Figure 3.37 shows the current in the transformer primary windings T1' and T1. It's important to see as the peak value of the current in the primary windings T1' is approximately 250 A. Figures 3.38 and 3.39 show the voltage of the transformer primary windings $\mathrm{T} 1$ ' and $\mathrm{T} 1$ and of secondary windings; it can be notice that transformer works well. While is fundamental to see the voltage on the rectifier diodes. It possible understands as the voltage stress on the diodes is more less in the Graetz bridge than in the configuration with center-tapped transformer. In fact as possible well to see in the Figure 3.42 the maximum voltage value on a rectifier diode is $1.4 \mathrm{kV}$, while the Figure 3.40 shows that the rectifier diode is submitted to an hard voltage stress with a voltage value that is perfectly double respect to Graetz bridge configuration.

Also an important particular is shown in the Figure 3.39. They represent the waveforms relatively at voltage across the transformer secondary windings in the Graetz bridge configuration of the rectifier. And so it is important to see as the voltage stress is not presented differently as shown in the Figure 3.41. Figure 3.43 represents the main cap bank voltage. The voltage increases in $100 \mathrm{~ms}$ from $1.3 \mathrm{kV}$ to $1.4 \mathrm{kV}$ and it discharges in $2 \mathrm{~ms}$ with a droop voltage of $100 \mathrm{~V}$. While as shown in the Figure 3.44 the main capacitor bank absorbs in $100 \mathrm{~ms}$ a constant charging current and so the converter is functioning in constant power; besides in $2 \mathrm{~ms}$ the capacitor supplies the load with a peak current of $1200 \mathrm{~A}$.

\subsubsection{Soft Switching Commutation}

Power semiconductors are the heart of switching mode power supplies. A soft switching commutation in several power converters it's important to reduce the conduction losses and switching losses. There are two main soft-switching approaches that are the zero-current switching (ZCS) and the zero-voltage switching (ZVS). The choice depends on the semiconductor device technology that will be used. For example, Mosfet's present better performance under ZVS. This is because under ZCS the capacitive turn-on losses increase the switching losses and the electromagnetic interference (EMI).

\subsection{Snubbers circuits}

To perform a soft switching commutation in the power converters, typically are used the snubber circuits [17]. Snubbers are circuits which are placed across the semiconductor devices to reduce the electrical stresses placed on a device during switching by a power electronics converter top levels that are within the electrical ratings of the device. More explicitly a snubber circuit reduces the switching stresses to safe levels by:

- Limiting voltages applied to devices during turn-off transients;

- Limiting device currents during turn-on transients; 


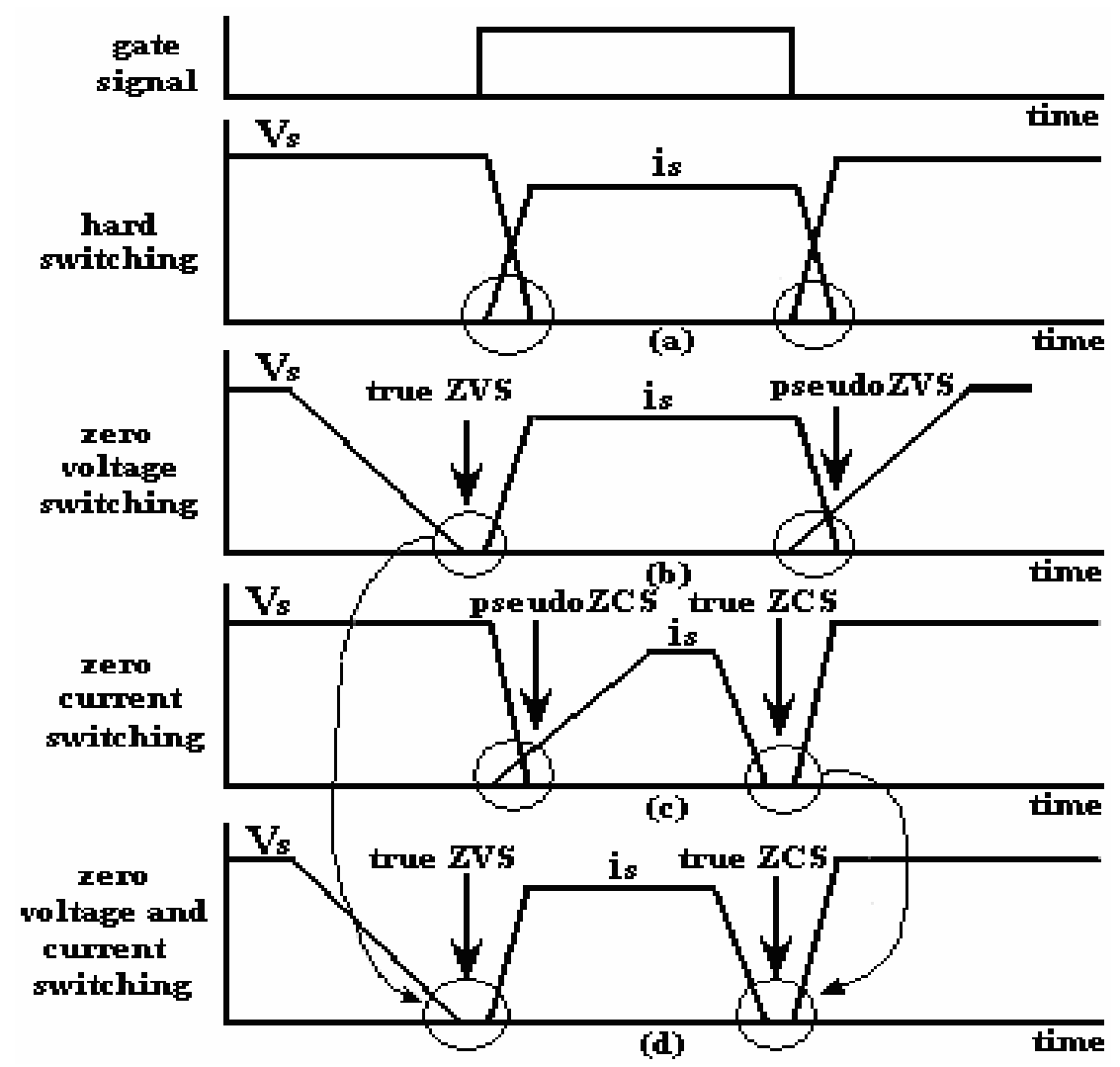

Figure 3.45: Commutation waveforms.

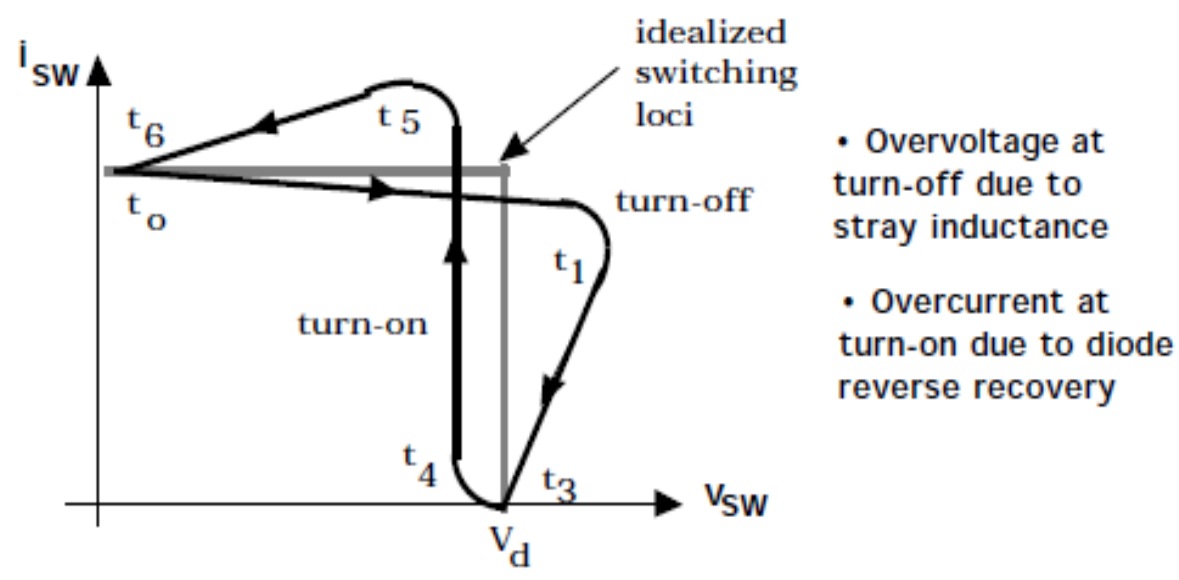

Figure 3.46: Switching loci relatively to hard turn-on and turn-off of a semiconductor device. 


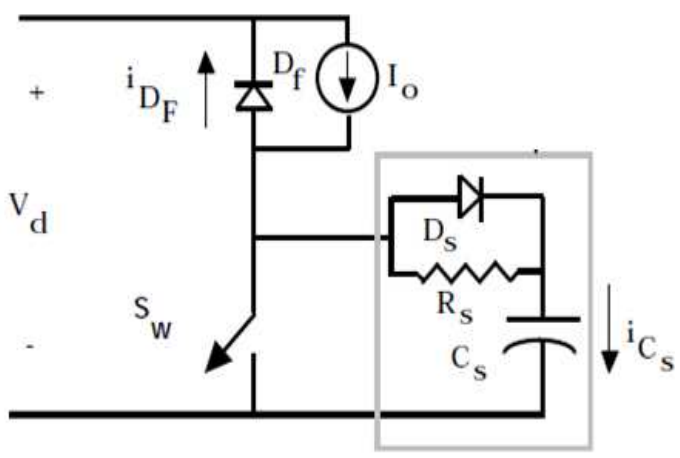

Figure 3.47: Circuit configuration of turn-off snubber

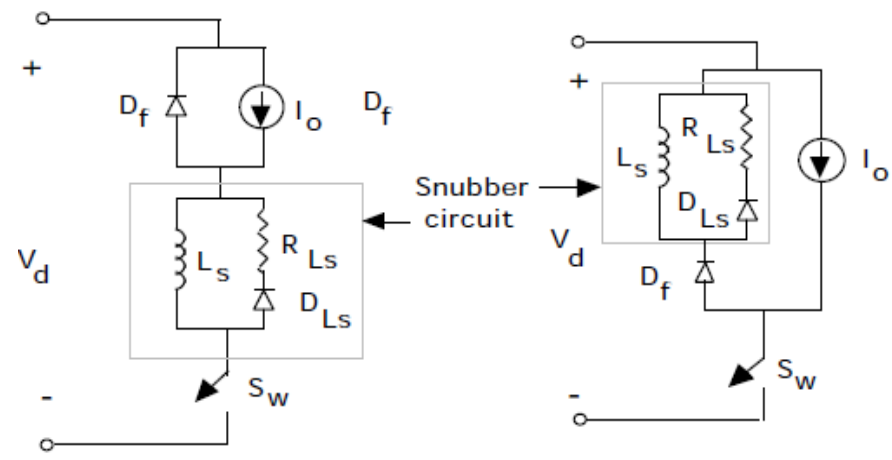

Figure 3.48: Circuit configuration of turn-on snubber.

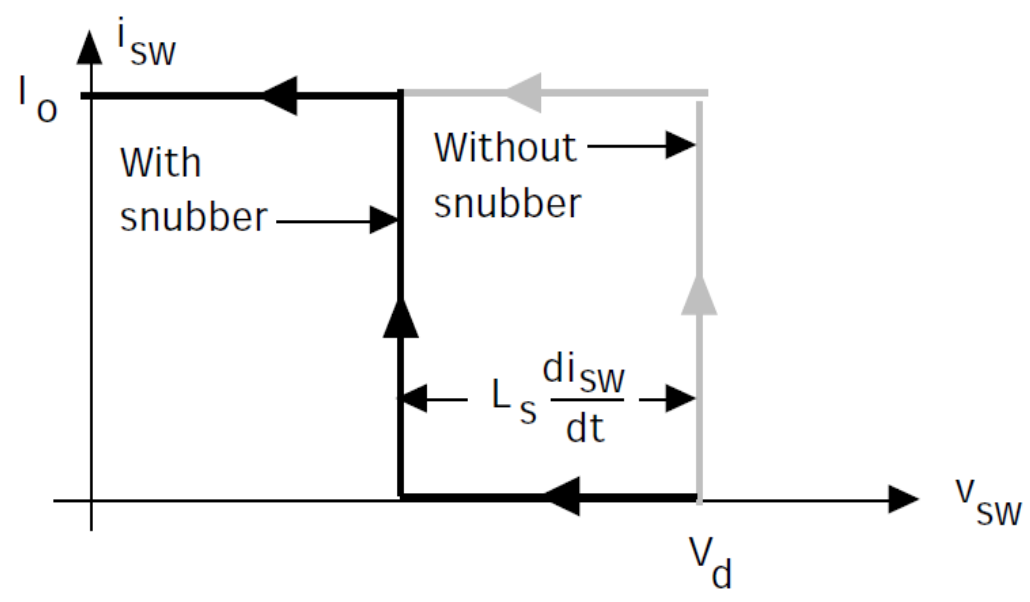

Figure 3.49: Switching trajectories with and without turn-on snubber. 

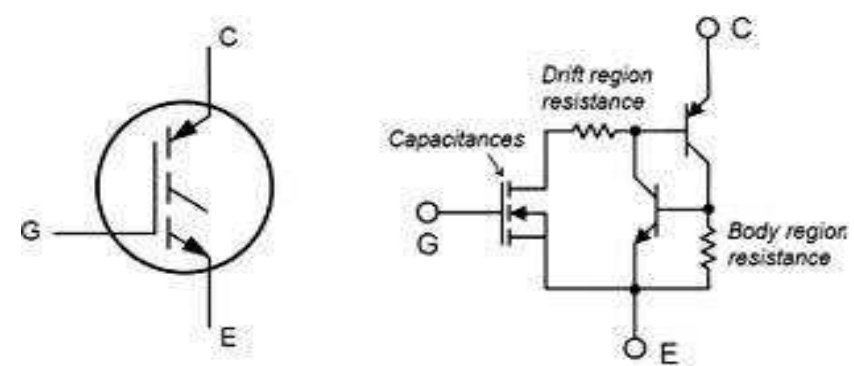

Figure 3.50: IGBT circuit symbol and equivalent circuit.

- Limiting the rate of rise $(d i / d t)$ of currents through devices at device turn-on;

- Limiting the rate of rise $(d v / d t)$ of voltages across devices during its turn-off or during reapplied forward blocking voltages (e.g., SCRs during the forward blocking state);

- Shaping of switching trajectory of the device as it turns on and off to keep the functioning of the devise inside its safe operating area (SOA);

- Limiting total losses due to switching;

- Limiting EMI by damping voltage and current ringing.

From circuit topology perspective, there are three broad classes of snubber circuits. These classes include:

- Unpolarized series R-C snubbers used to protect diodes and thyristors by limiting the maximum voltage and $d v / d t$ at reverse recovery;

- Polarized R-C snubbers. These snubbers are used to shape the turn-off portion of the switching trajectory of controllable switches, to clamp voltage applied to the device to safe levels, or to limit $d v / d t$ during device turn-off. The Figure 3.47 shows an example of this type of snubbers.

- Polarized L-R snubbers. These snubbers are used to shape the turn-on switching trajectory of controllable switches and/or to limit $d i / d t$ during device turn-on. The Figure 3.48 shows an example of this type of snubbers and the Figure 3.49 shows the shape of switching trajectory of the device using the turn-on snubber.

It must be emphasized that snubbers are not fundamental part of a power electronic converter circuit. The snubber circuit is an addition to a basic converter, which is added to reduce stresses on an electrical component, usually a power semiconductor device. Snubbers may be used singly or in combination depending on the requirements. The additional complexity and cost added to converter circuit by the presence of the snubber must be balanced against the benefits of limiting the electrical stresses on critical components. 
MITSUBISHI IGBT MODULES

\section{CM600HA-12H}

HIGH POWER SWITCHING USE INSULATED TYPE
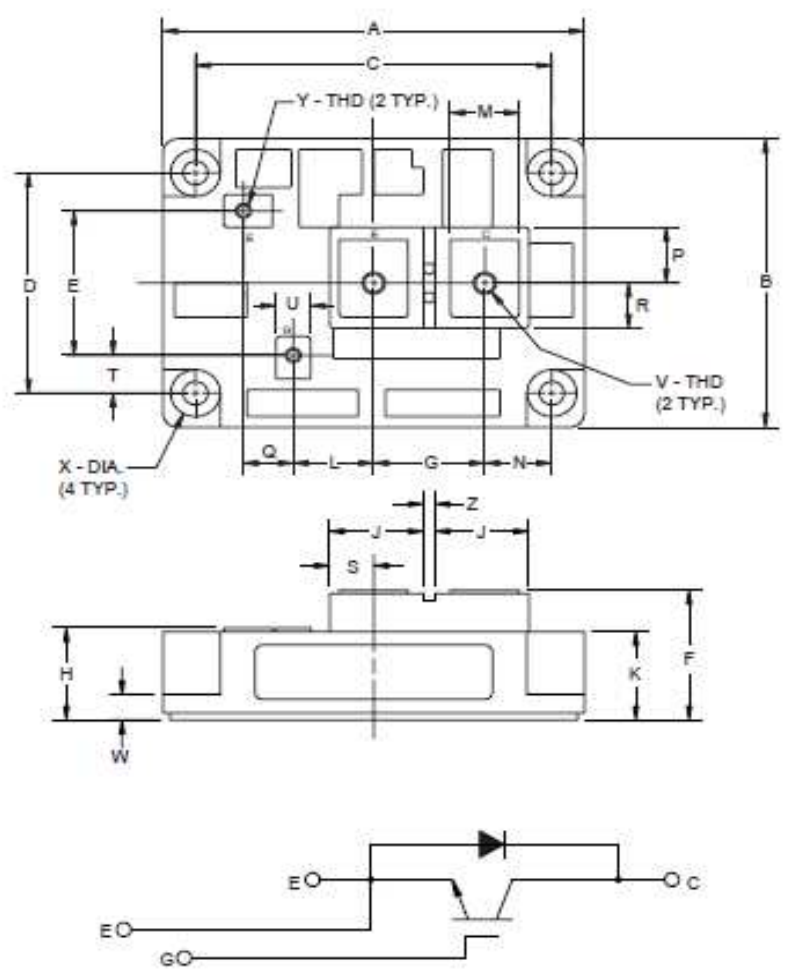

Outline Drawing and Circuit Diagram

\begin{tabular}{ccc}
\hline Dimensions & Inches & Millimeters \\
\hline $\mathrm{A}$ & 4.33 & 110.0 \\
\hline $\mathrm{B}$ & 3.15 & 80.0 \\
\hline $\mathrm{C}$ & $3.66 \pm 0.008$ & $93.0 \pm 0.25$ \\
\hline $\mathrm{D}$ & $2.44 \pm 0.008$ & $62.0 \pm 0.25$ \\
\hline $\mathrm{E}$ & 1.57 & 40.0 \\
\hline $\mathrm{F}$ & $1.42 \mathrm{Max}$. & $36.0 \mathrm{Max}$. \\
\hline $\mathrm{G}$ & 1.14 & 29.0 \\
\hline $\mathrm{H}$ & $1.00 \mathrm{Max}$. & $25.5 \mathrm{Max}$. \\
\hline $\mathrm{J}$ & 0.84 & 24.5 \\
\hline $\mathrm{K}$ & 0.83 & 24.0 \\
\hline $\mathrm{L}$ & 0.83 & 21.0 \\
\hline $\mathrm{M}$ & 0.71 & 18.0 \\
\hline
\end{tabular}

\begin{tabular}{ccc}
\hline Dimensions & Inches & Millimeters \\
\hline $\mathrm{N}$ & 0.80 & 17.5 \\
\hline $\mathrm{P}$ & 0.61 & 15.5 \\
\hline $\mathrm{Q}$ & 0.51 & 13.0 \\
\hline $\mathrm{R}$ & 0.49 & 12.5 \\
\hline $\mathrm{S}$ & 0.45 & 11.5 \\
\hline $\mathrm{T}$ & 0.43 & 11.0 \\
\hline $\mathrm{U}$ & 0.35 & 9.0 \\
\hline $\mathrm{V}$ & M8 Metric & M8 \\
\hline $\mathrm{W}$ & 0.28 & 7.0 \\
\hline $\mathrm{X}$ & 0.256 Dia. & Di3. 6.50 \\
\hline $\mathrm{Y}$ & M4 Metric & M4 \\
\hline $\mathrm{Z}$ & 0.12 & 3.0 \\
\hline
\end{tabular}

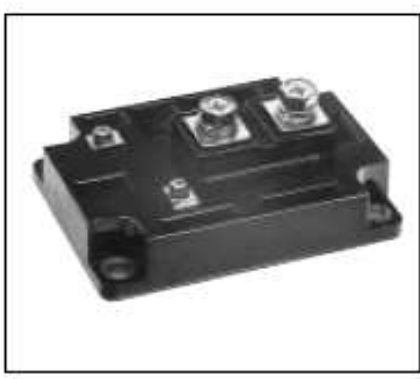

Description:

Mitsubishi IGBT Modules

are designed for use in switching applications. Each module consists of one IGBT in a single configuration with a reverse-connected super-fast recovery free-wheel diode. All components and interconnects are isolated from the heat sinking baseplate, offering simplified system assembly and thermal management.

\section{Features:}

$\square$ Low Drive Power

$\square$ Low $V_{C E \text { (sat) }}$

$\square$ Discrete Super-Fast Recovery Free-Wheel Diode

$\square$ High Frequency Operation

$\square$ Isolated Baseplate for Easy Heat Sinking

\section{Applications:}

\begin{tabular}{|c|c|c|}
\hline $\begin{array}{l}\square \mathrm{A} \\
\square \mathrm{N} \\
\square \mathrm{U} \\
\square \mathrm{V}\end{array}$ & $\begin{array}{l}\text { Motor Control } \\
\text { on/Servo Cor } \\
\text { ding Power S }\end{array}$ & plies \\
\hline $\begin{array}{l}\text { Ord } \\
\text { Exar } \\
\text { mod } \\
\text { the t } \\
12 \mathrm{H} \\
\text { pere }\end{array}$ & $\begin{array}{l}\text { g Informatio } \\
\text { Select the } \\
\text { number you } \\
\text { below -i.e. } \\
600 \mathrm{~V} \text { (V.CE } \\
\text { gle IGBT Mo }\end{array}$ & $\begin{array}{l}\text { mplete part } \\
\text { sire from } \\
1600 \mathrm{HA}- \\
600 \mathrm{Am}- \\
\text { le. }\end{array}$ \\
\hline Type & $\begin{array}{c}\text { Current Rating } \\
\text { Amperes }\end{array}$ & $\begin{array}{c}V_{\text {CES }} \\
\text { Volts }(x 50)\end{array}$ \\
\hline$\overline{\mathrm{CM}}$ & 600 & 12 \\
\hline
\end{tabular}


MITSUBISHI IGBT MODULES

CM600HA-12H

HIGH POWER SWITCHING USE INSULATED TYPE

\begin{tabular}{|c|c|c|c|}
\hline Ratings & Symbol & $\mathrm{CM} 600 \mathrm{HU}-12 \mathrm{H}$ & Units \\
\hline Junction Temperature & $\mathrm{T}_{\mathrm{J}}$ & -40 to 150 & ${ }^{\circ} \mathrm{C}$ \\
\hline Storage Temperature & $T_{5 t g}$ & -40 to 125 & ${ }^{\circ} \mathrm{C}$ \\
\hline Collector-Emitter Voltage (G-E SHORT) & $V_{\text {CES }}$ & 600 & Volts \\
\hline Gate-Emitter Voltage (C-E SHORT) & $V_{G E S}$ & \pm 20 & Volts \\
\hline Collector Current $\left(T_{C}=25^{\circ} \mathrm{C}\right)$ & $\mathrm{I}_{\mathrm{C}}$ & 600 & Amperes \\
\hline Peak Collector Current $\left(T_{\mid} \leq 150^{\circ} \mathrm{C}\right)$ & $\mathrm{I} \mathrm{CM}$ & $1200^{x}$ & Amperes \\
\hline Emitter Current ${ }^{x=}\left(T_{C}=25^{\circ} \mathrm{C}\right)$ & $\mathrm{lE}$ & 600 & Amperes \\
\hline Peak Emitter Current" & IEM & $1200^{x}$ & Amperes \\
\hline Maximum Collector Dissipation $\left(\mathrm{T}_{\mathrm{C}}=25^{\circ} \mathrm{C}\right)$ & $P_{c}$ & 2100 & Watts \\
\hline Mounting Torque, M8 Main Terminal & - & $8.83 \sim 10.8$ & $\mathrm{~N} \cdot \mathrm{m}$ \\
\hline Mounting Torque, M6 Mounting & - & $1.96 \sim 2.94$ & $\mathrm{~N}-\mathrm{m}$ \\
\hline Mounting Torque, M4 Terminal & - & $0.88 \sim 1.47$ & $\mathrm{~N}-\mathrm{m}$ \\
\hline Weight & - & 560 & Grams \\
\hline Isolation Voltage (Main Terminal to Baseplate, AC 1 min.) & $V_{i s o}$ & 2500 & Vrms \\
\hline
\end{tabular}

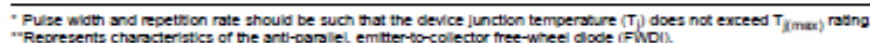

Static Electrical Characteristics, $\mathrm{T}_{\mathrm{j}}=25^{\circ} \mathrm{C}$ unless otherwise specified

\begin{tabular}{|c|c|c|c|c|c|c|}
\hline Characteristics & Symbol & Test Conditions & Min. & Typ. & Max. & Units \\
\hline Collector-Cutoff Current & $\mathrm{I}_{\mathrm{CES}}$ & $V_{C E}=V_{C E S} \cdot V_{G E}=O V$ & - & - & 1.0 & $\mathrm{~mA}$ \\
\hline Gate Leakage Current & $I_{\text {GES }}$ & $V_{G E}=V_{G E S} \cdot V_{C E}=0 V$ & - & - & 0.5 & $\mu \mathrm{A}$ \\
\hline Gate-Emitter Threshold Voltage & $V_{G E(t h)}$ & $I_{C}=60 \mathrm{~mA}, V_{C E}=10 \mathrm{~V}$ & 4.5 & 6.0 & 7.5 & Volts \\
\hline \multirow[t]{2}{*}{ Collector-Emitter Saturation Voltage } & $\mathrm{V}_{\mathrm{CE} \text { (sat) }}$ & $\mathrm{I}_{\mathrm{C}}=600 \mathrm{~A}, \mathrm{~V}_{\mathrm{GE}}=15 \mathrm{~V}$ & - & 2.1 & $2.8^{n}$ & Volts \\
\hline & & $I_{C}=600 A, V_{G E}=15 V, T_{J}=150^{\circ} \mathrm{C}$ & - & 2.15 & - & Volts \\
\hline Total Gate Charge & $Q_{G}$ & $V_{C C}=300 \mathrm{~V}, I_{C}=600 A, V_{G E}=15 \mathrm{~V}$ & - & 1800 & - & $\mathrm{nC}$ \\
\hline Emitter-Collector Voltage & $V_{E C}$ & $\mathrm{l}_{\mathrm{E}}=600 \mathrm{~A}, \mathrm{~V}_{\mathrm{GE}}=0 \mathrm{~V}$ & - & - & 2.8 & Volts \\
\hline
\end{tabular}

" Pube widh and repettion rate should be such that device Junction temperaure rise is negl gitie

Dynamic Electrical Characteristics, $\mathrm{T}_{\mathrm{j}}=25^{\circ} \mathrm{C}$ unless otherwise specified

\begin{tabular}{|c|c|c|c|c|c|c|c|}
\hline \multicolumn{2}{|c|}{ Characteristics } & Symbol & Test Conditions & Min. & Typ. & Max. & Units \\
\hline \multicolumn{2}{|c|}{ Input Capacitance } & $\mathrm{C}_{\mathrm{les}}$ & & - & - & 60 & $\mathrm{nF}$ \\
\hline \multicolumn{2}{|c|}{ Output Capacitance } & $\mathrm{C}_{\text {oes }}$ & $V_{G E}=O V, V_{C E}=10 \mathrm{~V}$ & - & - & 21 & $\mathrm{nF}$ \\
\hline \multicolumn{2}{|c|}{ Reverse Transfer Capacitance } & $\mathrm{C}_{\text {res }}$ & & - & - & 12 & $\mathrm{nF}$ \\
\hline Resistive & Turn-on Delay Time & $\operatorname{tg}(0 n)$ & & - & - & 350 & ns \\
\hline Load & Rise Time & $t_{r}$ & $V_{C C}=300 \mathrm{~V}, l_{C}=600 \mathrm{~A}$ & - & - & 700 & ns \\
\hline Switching & Turn-off Delay Time & $\operatorname{tg}(0 \pi)$ & $V_{G E 1}=V_{G E 2}=15 V, R_{G}=1.0 \Omega$ & - & - & 350 & ns \\
\hline Times & Fall Time & $t_{f}$ & & - & - & 300 & ns \\
\hline \multicolumn{2}{|c|}{ Diode Reverse Recovery Time } & $t_{\pi}$ & $\mathrm{I}_{\mathrm{E}}=600 \mathrm{~A}, \mathrm{di}_{E} / \mathrm{dt}=-1200 \mathrm{~A} / \mu \mathrm{s}$ & - & - & 110 & ns \\
\hline \multicolumn{2}{|c|}{ Diode Reverse Recovery Charge } & $Q_{\pi}$ & $\mathrm{I}_{\mathrm{E}}=600 \mathrm{~A}, \mathrm{di} E / \mathrm{dt}=-1200 \mathrm{~A} / \mu \mathrm{s}$ & - & 1.62 & - & $\mu \mathrm{C}$ \\
\hline
\end{tabular}

Thermal and Mechanical Characteristics, $\mathrm{T}_{\mathrm{j}}=25^{\circ} \mathrm{C}$ unless otherwise specified

\begin{tabular}{|c|c|c|c|c|c|c|}
\hline Characteristics & Symbol & Test Conditions & Min. & Typ. & Max. & Units \\
\hline Thermal Resistance, Junction to Case & $R_{\text {th }(1-c)}$ & Per IGBT & - & - & 0.06 & ${ }^{\circ} \mathrm{C} / \mathrm{W}$ \\
\hline Thermal Resistance, Junction to Case & $R_{\text {th }(\mid-c)}$ & Per FWDi & - & - & 0.12 & ${ }^{\circ} \mathrm{C} / \mathrm{W}$ \\
\hline Contact Thermal Resistance & $R_{\text {th }}(\infty-1)$ & Per Module, Thermal Grease Applied & - & - & 0.035 & ${ }^{\circ} \mathrm{C} / \mathrm{W}$ \\
\hline
\end{tabular}

Figure 3.51: Datasheet of CM600HA-12H Power IGBT. 
Klystron Modulator Power Supplies

\begin{tabular}{|r|r|r|r|r|r|}
\hline Csnubber [nF] & Pigbt1 [W] & Pigbt2 [W] & Pigbt3 [W] & Pigbt4 [W] & Ptot [W] \\
\hline 120 & & & & & \\
\hline 300 & 1200 & 1290 & 210 & 278,5 & 2978,5 \\
\hline 500 & 1088 & 1215 & 124,05 & 201,9 & 2683,95 \\
\hline 800 & 1021 & 1161 & 84,94 & 167,65 & 2501,59 \\
\hline 1000 & 1000 & 1075 & 70,21 & 144,7 & 2336,91 \\
\hline 2000 & 923 & 1015 & 63,4 & 140 & 2278,4 \\
\hline
\end{tabular}

Table 3.52: Mean power losses and total power losses values depending of snubber capacity.

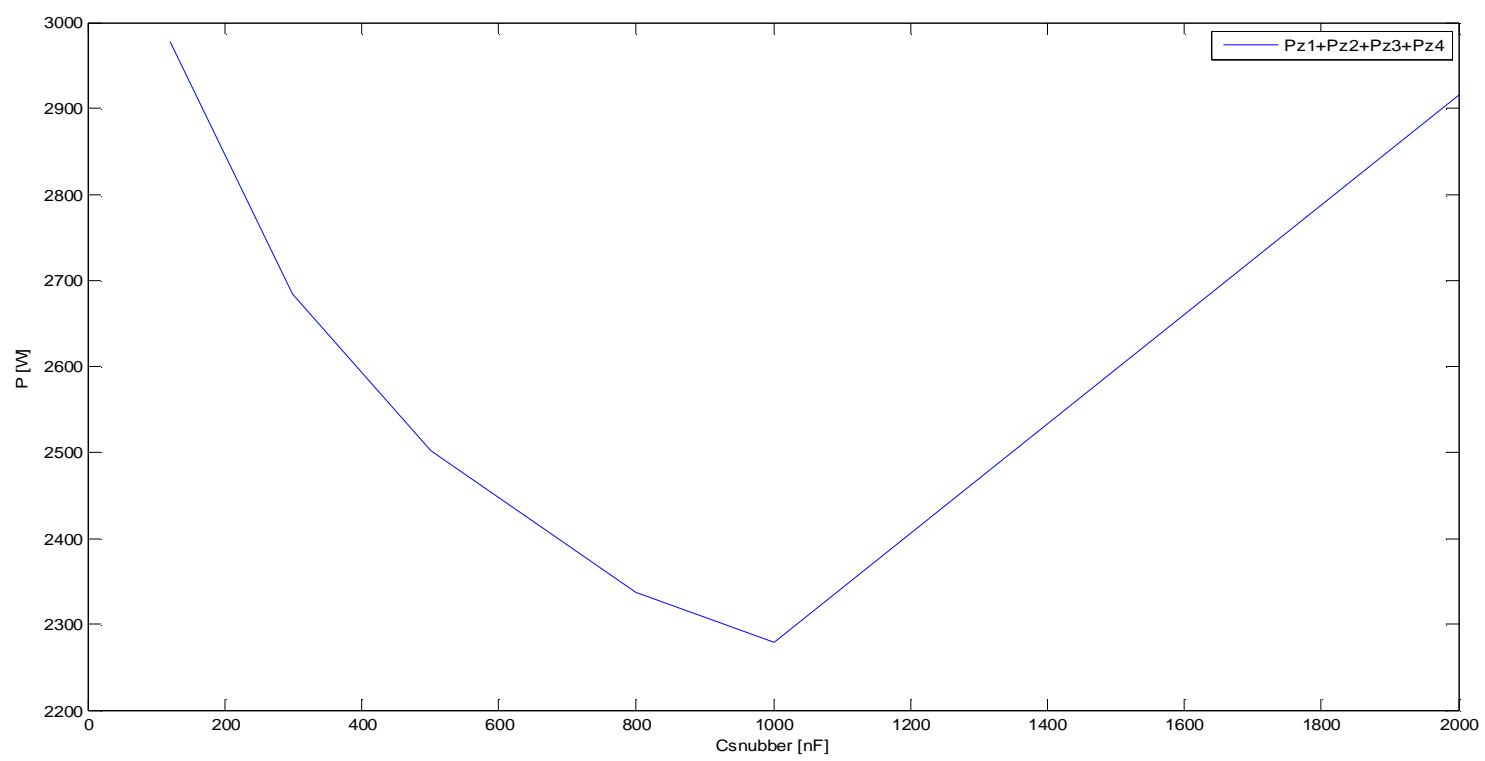

Figure 3.53: Total power losses depending of snubber capacity.

\subsubsection{Select of SILC's switches}

This paragraph will explain what type of switch was selected for the project. In particular, after a short description of the switch, will be shown the datasheet of device, the selection of the snubber circuit in particular the calculation of snubber capacitance and in the end will be illustrated the commutation waveforms of switches to verified if the soft switching requirement is satisfied. 
Klystron Modulator Power Supplies

\subsection{Insulated Gate Bipolar Transistors ( IGBTs)}

As previously illustrated, we have need of four switches that work with high values of voltage and current and with a low switching frequency $(15 \mathrm{kHz})$. For this reasons, IGBT switches was chosen. The circuit symbol and equivalent circuit for an IGBT is shown in Figure 3.50.

The IGBTs have some advantages of the MOSFETs (Metal-Oxide-Semiconductor FieldEffect Transistor), the BJT (Bipolar Junction Transistor), and the GTO (Gate Turn-On Thyristor) combined. Similar to the MOSFET, the IGBT has a high impedance gate, which requires only a small amount of energy to switch the device. Like the BJT, the IGBT has a small on-state voltage even in devices with large blocking voltage ratings (for example, $\mathrm{V}_{\text {on }}$ is 2-3 V in $1000 \mathrm{~V}$ device). While similar to the GTO, IGBTs can be designed to block negative voltages, as their idealized switch characteristics. Besides, insulated gate bipolar transistors have turn-on and turn-off times on the order of $1 \mu \mathrm{s}$ and are available in module ratings as large as $1700 \mathrm{~V}$ and $1200 \mathrm{~A}$. Voltages ratings of up to $3-4 \mathrm{kV}$ are projected and utilized. In the SILC project was utilized the Spice Model of CM600HA-12H, the Mitsubishi IGBT modules with a collector-emitter voltage of $600 \mathrm{~V}$ and a collector current of $600 \mathrm{~A}$. The datasheet of device is proposed [18].

\subsection{Choose of Snubber Capacity and Switching Commutations}

The aim of this paragraph is explain the procedure for the selection of the the snubber capacity to guarantee the soft switching of devices in their turn-offs. The particular procedure that was followed is to fix a value of snubber capacity and after a simulation take the value of mean power loss of a single device considering the turn-on and turn-off. The dates concerning this aspect are listed in the first four columns of the Table 3.51, while the last column, represent for a particular value of snubber capacity, the total mean power loss of all devices. Besides it's important to observe that the topology of SILC converter produce always in any different case a major stress on the switches of the low half bridge ( IGBT1 and IGBT2). In fact the Table 3.52 shows well as the main part of switching power losses derive from these switches. In the end, considering the Figure 3.53 the value of snubber capacity that was chosen for the project is $\mathrm{Cs}=1000 \mathrm{nF}$.

Figures 3.54, 3.55, 3.56, 3.57, 3.58, 3.59, 3.60 and 3.61 show the turn-on and turn-off commutations of IGBTs and in particular it's important to see as ZVS and ZCS commutation on all IGBTs are guaranteed. 


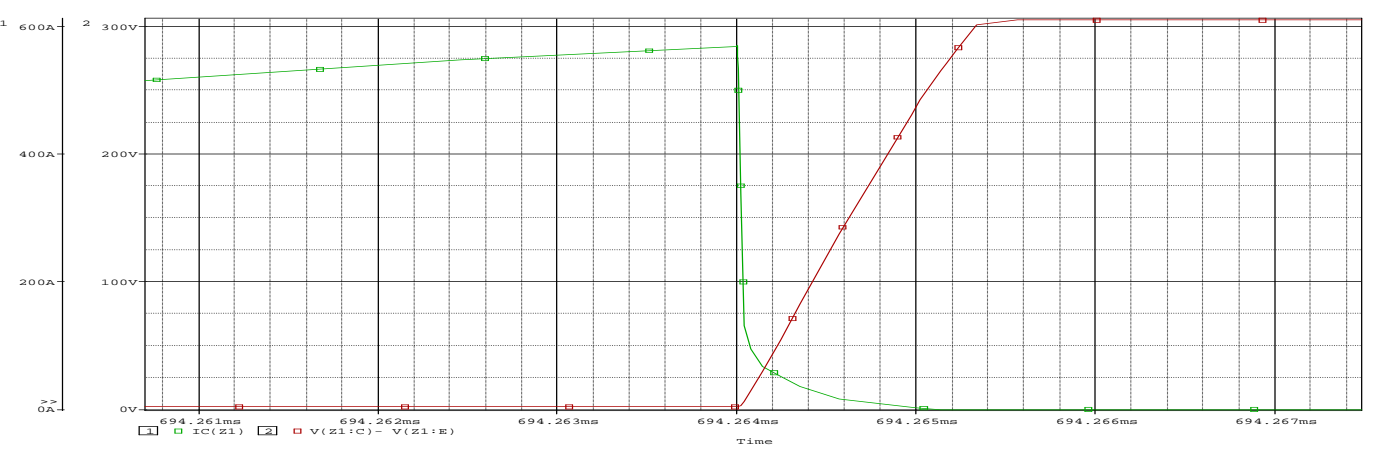

Figure 3.54: IGBT1 turn-off.

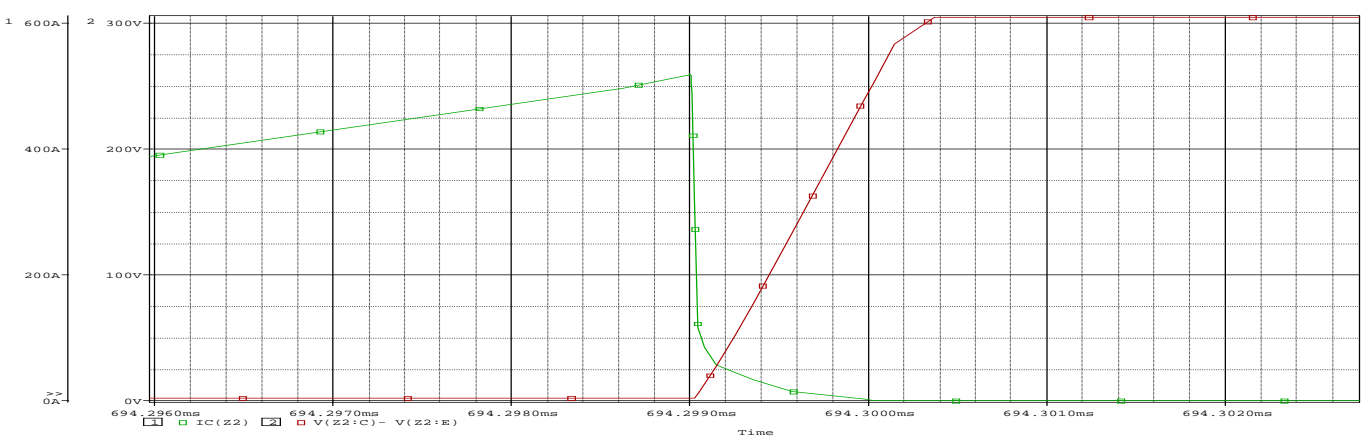

Figure 3.55: IGBT2 turn-off.

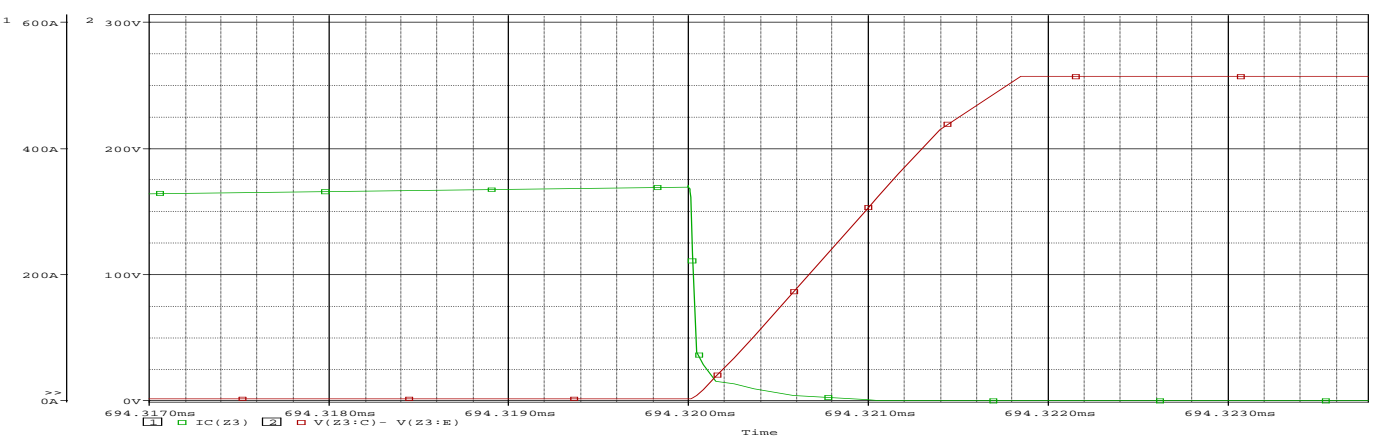

Figure 3.56: IGBT3 turn-off

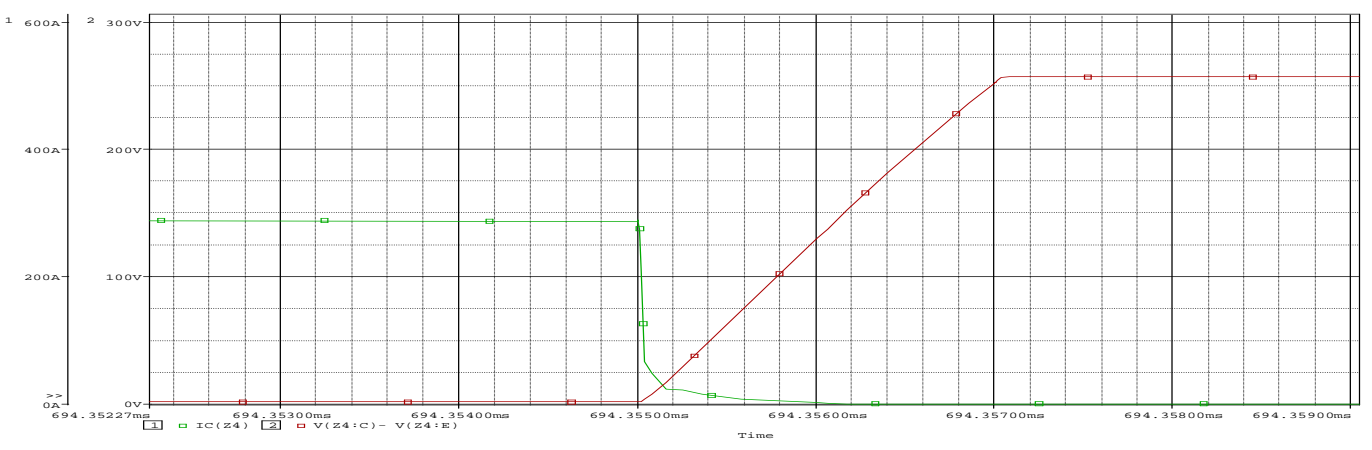

Figure 3.57: IGBT4 turn-off 


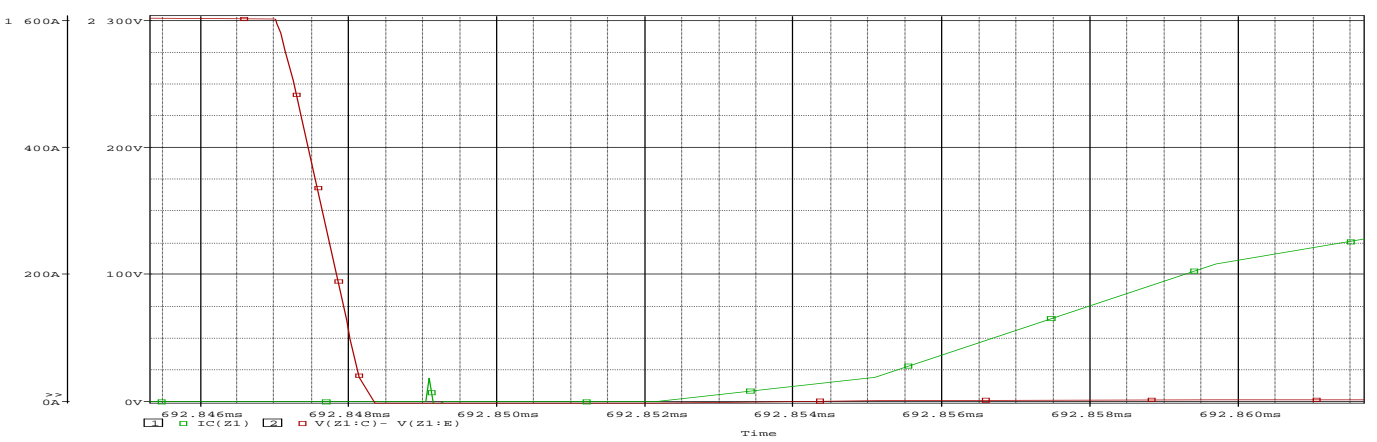

Figure 3.58: IGBT1 turn-on.

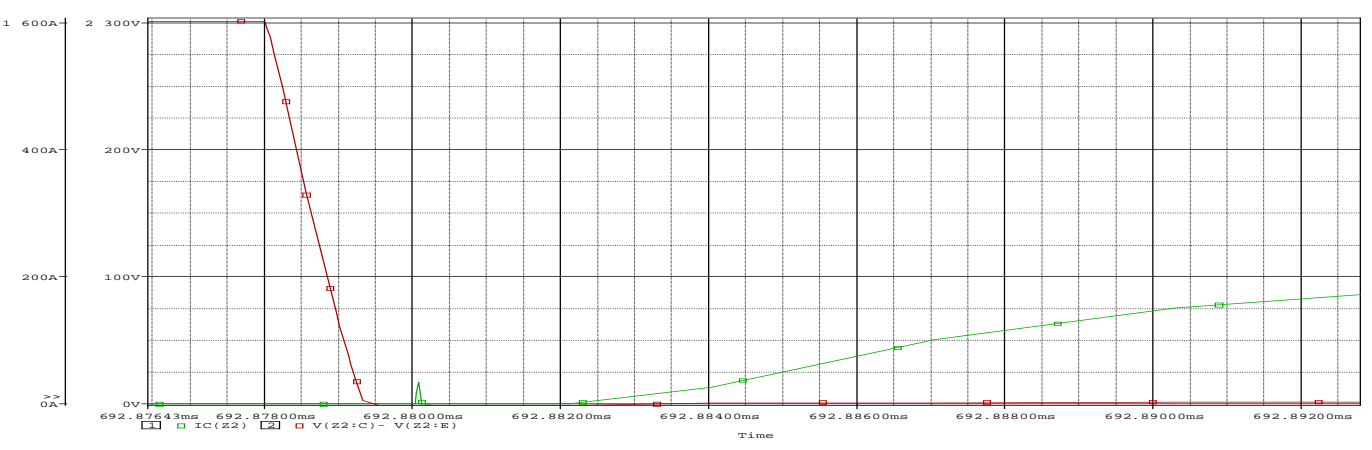

Figure 3.59: IGBT2 turn-on.

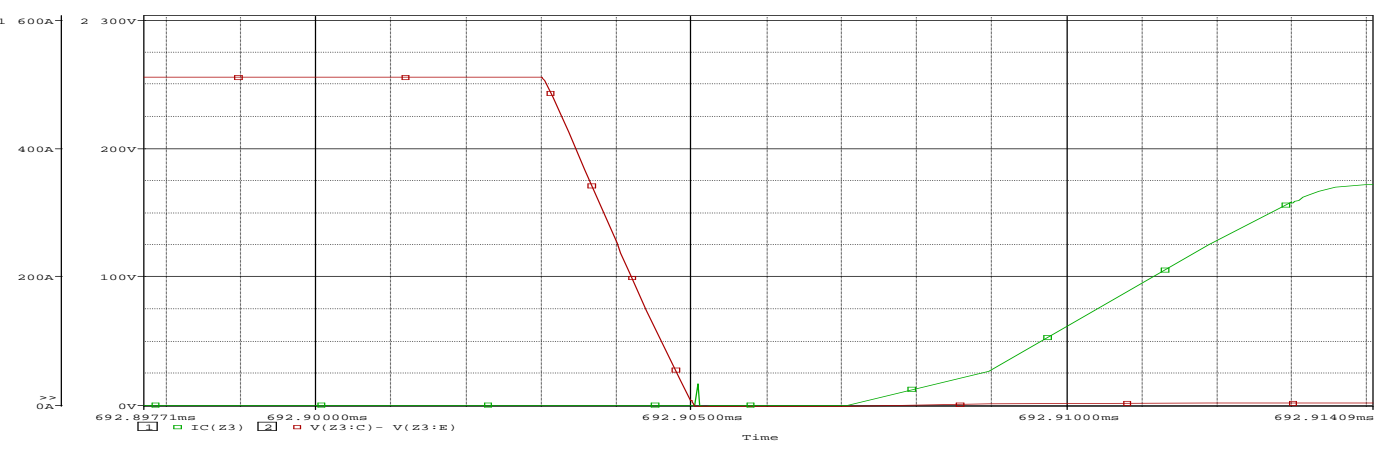

Figure 3.60: IGBT3 turn-on.

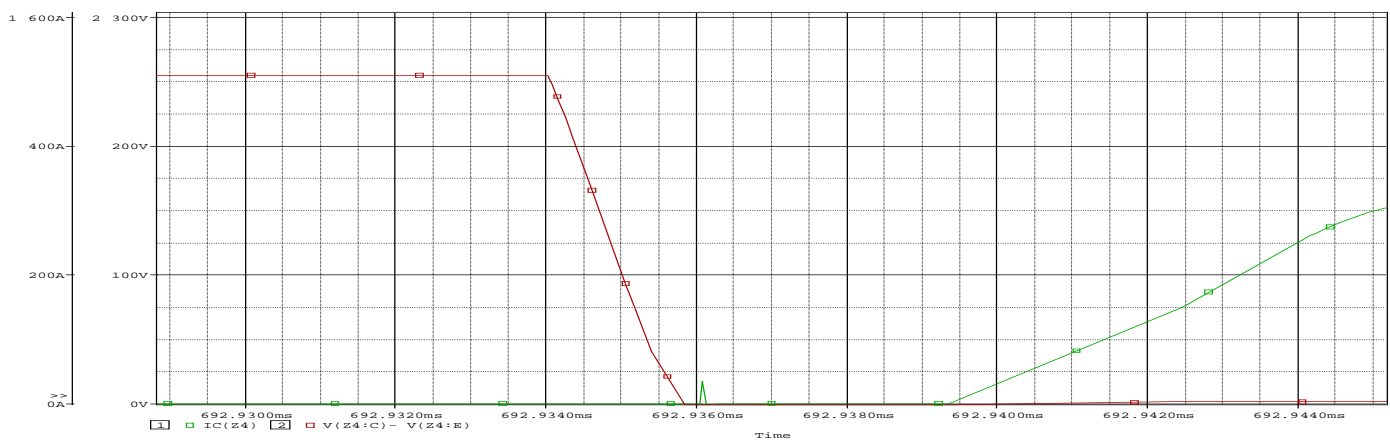

Figure 3.61: IGBT4 turn-on. 


\section{Chapter 4}

\section{A Solid State Marx Modulator for ILC}

\subsection{Introduction}

The Main Linac of the International Linear Collider will require, in order to accelerating electrons and positron up to center of mass energy of $500 \mathrm{GeV}$, per collision, 576 Radio Frequency (RF) stations. Each RF station will power a $10 \mathrm{MW}$ L-band klystron that will provide the acceleration energy to 26 niobium superconducting cavities. Each cavity has a design accelerating gradient of $35 \mathrm{MeV} / \mathrm{m}$. The $10 \mathrm{MW}$ L-band klystron will be supplied by a modulator which have to be capable to produce an output pulsed signal with a width of $2 \mathrm{~ms}$, a repetition rate of $10 \mathrm{~Hz}$, an amplitude of $120 \mathrm{kV}$ for a current of $120 \mathrm{~A}$. These design parameters correspond to output energy of about $27 \mathrm{~kJ}$. The output pulse droop have to stay under the nominal value of about $1 \%$. In the existing Fermilab Baseline Conceptual Design (BCD), the klystron modulator, generally called Bouncer Modulator, has a transformer-based topology. The Bouncer Modulator was developed at the Fermi National Accelerator Laboratory (FNAL) near to Batavia, Illinois (USA). It will be tested, starting from summer 2010, in the Fermilab ILC Test Facility at New Muon Lab (Figure 4.1.a, 4.1.b and Figure 4.2). The large size, weight, and cost of this transformer, the large capacitors banks as well as the large and expensive oil cooled inductor have been between the motivations of the present R\&D work, where we want to find alternative topologies that do not employ the use of all these massive and expensive components. In order to fit the ILC modulator design requirements, we developed a Solid State Marx Modulator $\left(\mathrm{SM}^{2}\right)$ that uses solid state switches (IGBTs) and isolation elements to connect capacitors in parallel, while charging and in series during the capacitor discharge.

\subsection{The Fermilab Bouncer Modulator}

The Bouncer Modulator is a transformer based large inductor modulator, developed by the Fermilab Accelerator Division [26, 27, 28, 29, 30]. The main layout of this apparatus is shown in Figure 4.3. This modulator uses a large size switched capacitor bank of $1.4 \mathrm{mF}$, that droops approximately of $20 \%$ during the full pulse width. This very large droop is then compensated by using a resonant LC circuit. If the capacitor bank is connected to the high side of the pulse transformer primary, using a series of GTO switches, instead, the resonant circuit is connected to the low side of the pulse transformer primary. The resulting output pulse is flat within $1 \%$ for $1.9 \mathrm{~ms}$ of the full $2.3 \mathrm{~ms}$ pulse width, fitting the design request. 

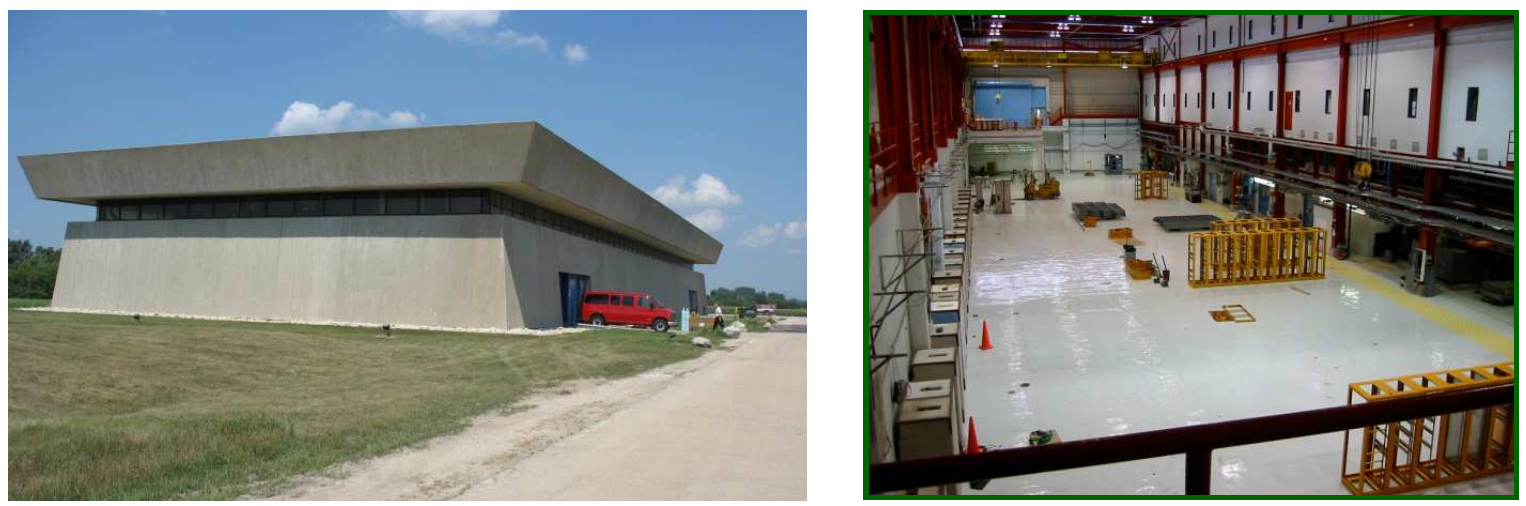

Figure 4.1: a) The New Muon Lab building; b) the ILC Test Facility at the New Muon Lab.

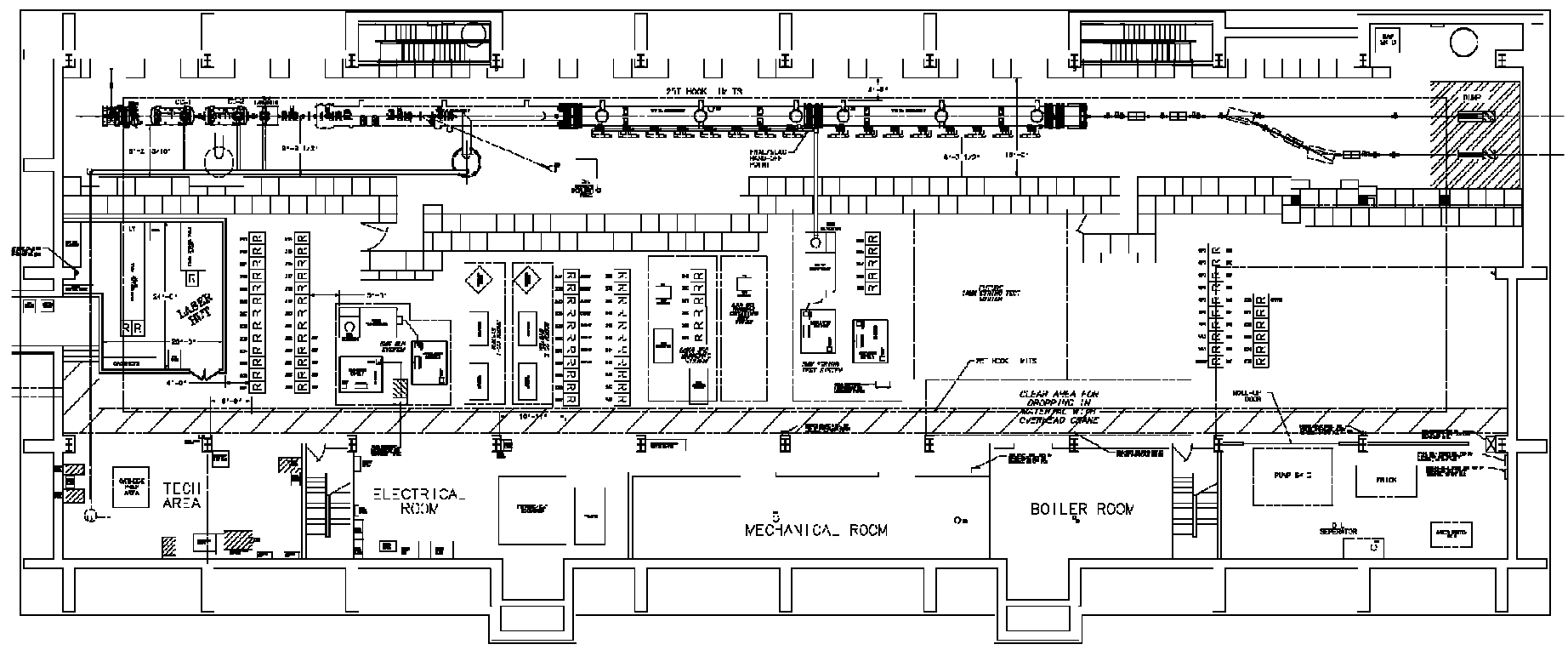

Figure 4.2: Layout of the beam line and service apparatus: cooling, RF lines, klystron, Modulator, etc. in the New Muon Lab complex. 
The Fermilab Bouncer Modulator has been tested and it is efficiency measured, from breaker to klystron, including energy losses, in the rise time. These measurements show that the Bouncer Modulator has an efficiency of $85 \%$.

\subsubsection{Operational Principle}

The Bouncer Modulator operational principle is the following. The main capacitor bank, $\mathrm{C}_{1}$, is charged approximately at a value that is $10 \%$ higher than what required output voltage, while the bouncer circuit elements, $\mathrm{L}_{3}(600 \mu \mathrm{H})$ and $\mathrm{C}_{2}(2 \mathrm{mF})$, in Figure 4.3, are charged to $10 \%$ of the required output voltage. In order to produce a long output pulse width, the bouncer resonant circuits switch $\mathrm{S}_{4}$, is closed, and the capacitor starts to ring with the inductor. At the appropriate time, the GTO switch, $S_{1}$, connecting the main capacitor bank to the pulse transformer, is closed. Then, after $2 \mathrm{~ms}$, the GTO switch is opened. The bouncer circuit then continues to ring through is full cycle.

\subsubsection{The Bouncer Modulator key components and costs}

The Fermilab Bouncer Modulator is done by several components that are custom. A reliable and robust closing and opening switches are required, so a series stack of six Gate Turn Off Thyristors (GTO) are used. Recently, IGBTs switches are used as a replacement for GTOs switches because of their higher reliability, lower cost and better switch control [27]. Figure 4.4 shows the Bouncer Modulator with a series stack of seven IGBTs. The bouncer circuit must be designed with low loss and the maximum impedance consistent with effective output regulation. The main capacitor bank must be designed for a crowbar operation and capacitor fault conditions. The pulse transformer (with 1:12 turns ratio) must have a very large volt second rating and a relatively low leakage inductance $(317 \mu \mathrm{H})$. Moreover, mechanical vibration, from the system, must be kept to a minimum because the RF superconducting cavities are nearby. The cost for the Bouncer Modulator components have been of about 370 $\mathrm{k} \$$. This prize includes approximately $20 \mathrm{k} \$$ spent for buying the control system, $50 \mathrm{k} \$$ for the GTO switches, $75 \mathrm{k} \$$ for the $400 \mathrm{~V}$ AC phase controller and for the power transformer and $90 \mathrm{k} \$$ for the pulse transformer. Fermilab facility, testing and the labor costs have not been included in the present list of the costs. 


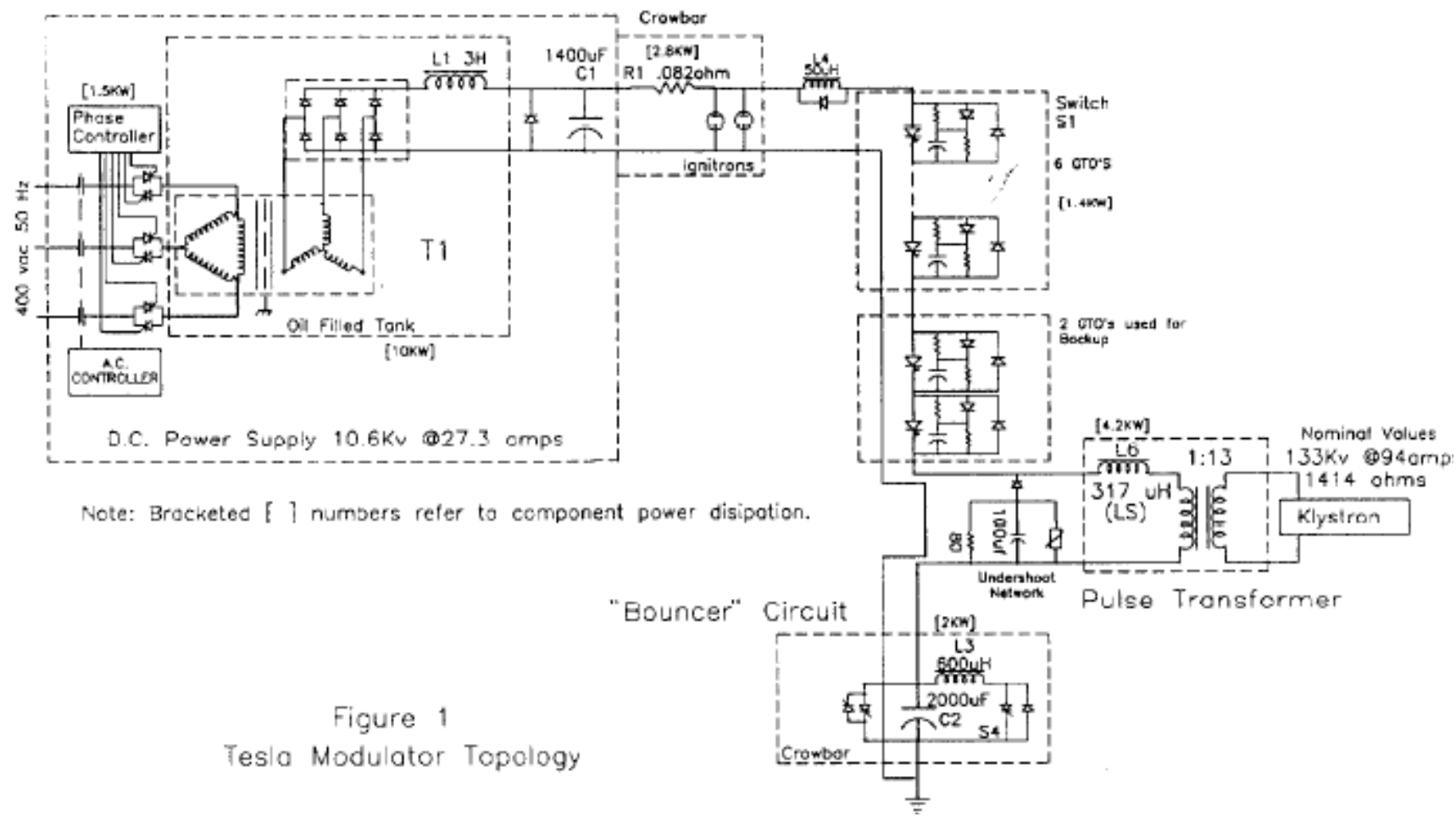

Figure 4.3: DC power supply and bouncer modulator design with GTOs switches.

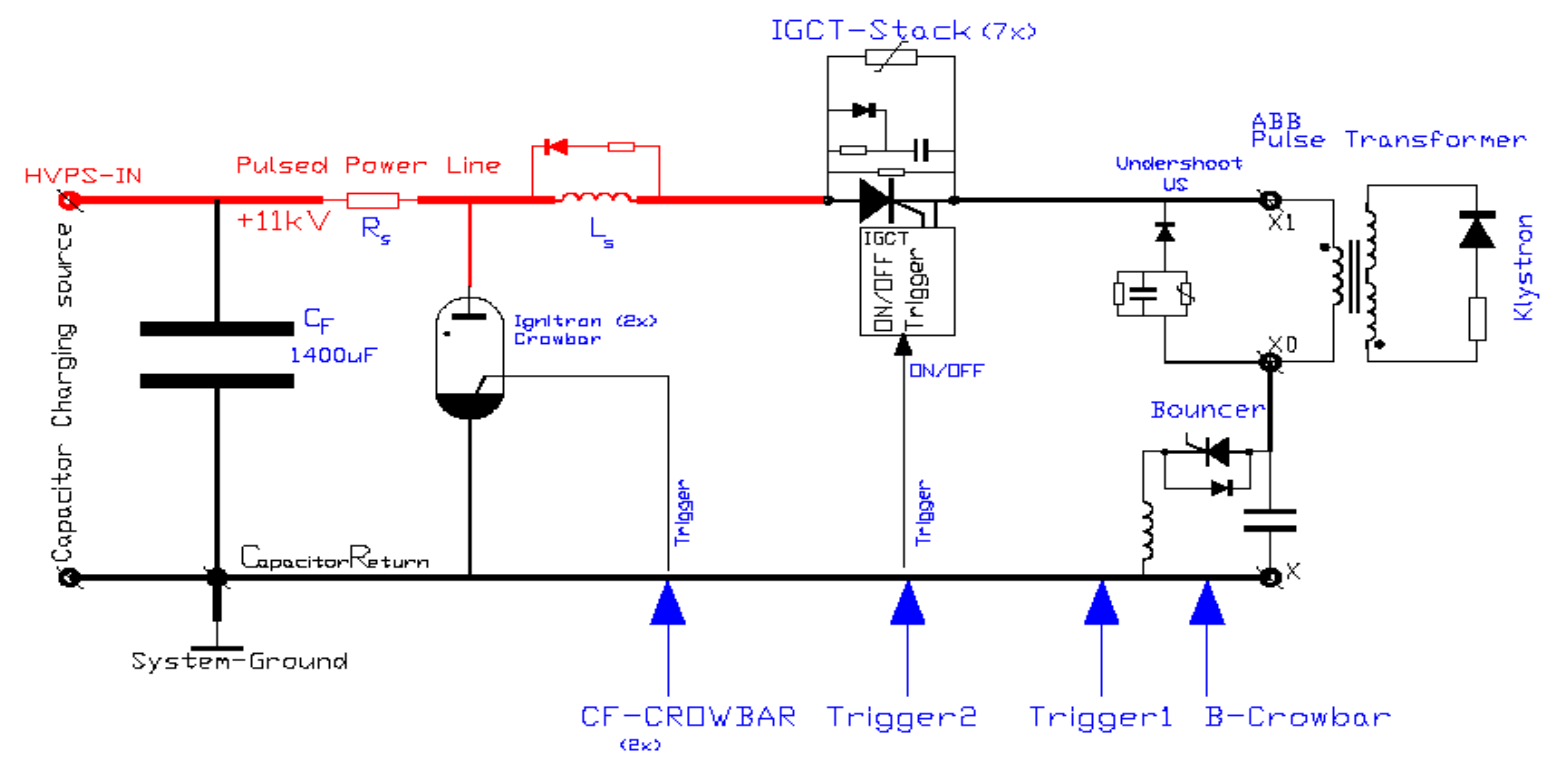

Figure 4.4: The Fermilab Bouncer Modulator design with IGBTs switches. 


\subsection{The Marx Generator Concept}

\subsubsection{Introduction}

A Marx generator is an electrical circuit, introduced for the first time by Erwin Otto Marx in 1924, whose purpose is to generate high-voltage pulses. In a Marx generator, a set of $N$ capacitors are charged in parallel to a low input voltage, $\mathrm{V}_{\mathrm{in}}$, and discharged in series to apply a high voltage $\boldsymbol{V}_{\text {out }}=\boldsymbol{N} \boldsymbol{V}_{\text {in }}$ to the target load. Marx generators are widely used in pulsed power technologies due to their simplicity, scalability and low voltage input requirements. Applications include medical accelerators, welding and cutting, laser supplies, radar systems and particle accelerators. Emerging uses include the sterilization of liquids and foods (via electroporation) [19,20], juice extraction [21], rock crushing [22], electromagneticallypowered transportation [23]. Marx generators are extensively used also for simulating the effects of lightning during high voltage and for aviation equipment testing. Recently, a bank of 36 Marx generators have been used by the Sandia National Laboratories, located in Albuquerque, New Mexico (USA), to generate X-rays in their so called Z Machine. The Z machine is the largest $X$ ray source in the world. It had been designed to test materials in conditions of extreme temperatures and pressures. This machine can be used also as an ignition switch for thermonuclear devices. At the present, Marx generators are also used in order to produce short high-power pulses for Pockels cells, for driving a Transversely Excited Atmospheric Pressure (TEA) Laser, for the ignition of conventional explosive used in the nuclear weapons, and to produce radar pulses. The time speed of such a kind of devices is relatively large, as the switching times, of even high-speed versions, is greater than $1 \mathrm{~ns}$ when many low-power electronic devices are faster. In the design of high-speed circuits, classical electrodynamics effects may be important; this is not the case of Marx generators, where the basic design is nevertheless essentially an electrostatic one. As matter of fact, if the first gap breaks down, pure electrostatic theory predicts that the voltage across all stages rises. However, stages are coupled capacitively to ground and serially to each other, and thus each stage encounters a voltage rise that is increasingly weaker the further the stage is, from the switching one. The adjacent stage, to the switching one, therefore, encounters the largest voltage rise, and thus switches in turn. As more stages switch, the voltage rise to the remainder increases, which speeds up their operation. Thus a voltage rise fed into the first stage becomes amplified and steepened at the same time. The speed of a switch is determined by the speed of the charge carriers, which gets higher with higher voltages and by the current 


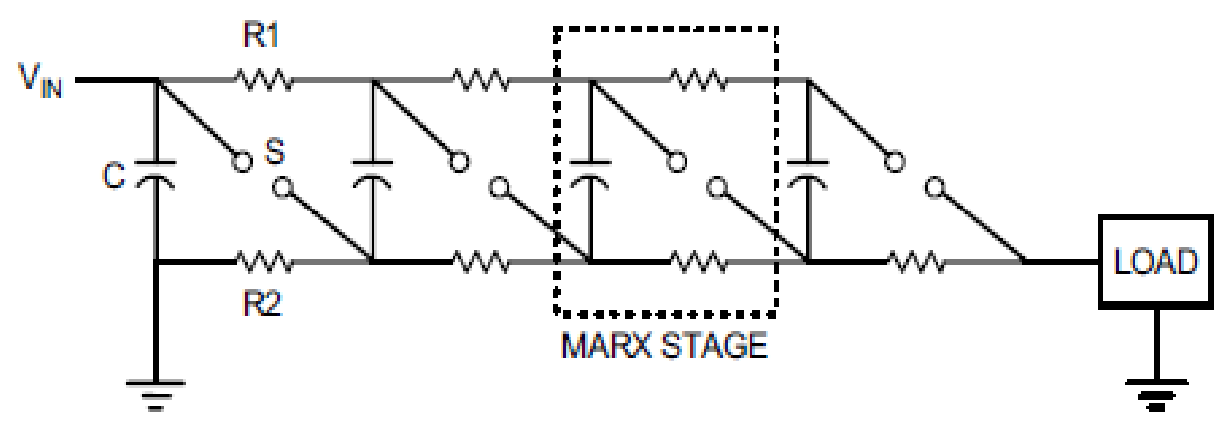

Figure 4.5: A classical 4-stages Marx generator circuit.

available to charge the inevitable parasitic capacity. In solid-state avalanche devices, a high voltage automatically, leads to high currents. Because the high voltage is applied, only for a short amount of time, solid-state switches will not heat up excessively. As compensation, for the higher voltages encountered, the later stages have to carry lower charge too. Stage cooling and capacitor recharging also go well together.

\subsubsection{The classical layout}

A Marx generator, in his classical configuration, has, essentially, a resistive-based topology. Figure 4.5 shows an example of a 4-stages classical Marx generator circuit. The operation principle of this design is characterized by a capacitor $\mathrm{C}$, which charges through two resistances, $R_{1}$ and $R_{2}$, when the spark gaps $S$ are opened. When fully charged, the lowest gap breaks down from overvoltage (or an external trigger), which puts the lowest two capacitors in series and then over-voltages the second gap and so on. Eventually, the erected load voltage is equal to the number of stages $\mathrm{N}$, multiplied by the input voltage $\mathrm{V}_{\text {in }}$. Likewise, the total erected capacitance, at that point, is equal to $\mathrm{C} / \mathrm{N}$. The major advantages of this topology are low voltage input requirements, while disadvantages are energy losses through passive charging elements as well as through charging/discharging stacked stray capacitances. Further issues may arise from stacking such as deformation of the pulse shape at higher currents, due to increased parasitic inductances. Charging resistors may also limit the pulse fall time as they act as high resistance shunt, through which stray capacitances must discharge. 
a)

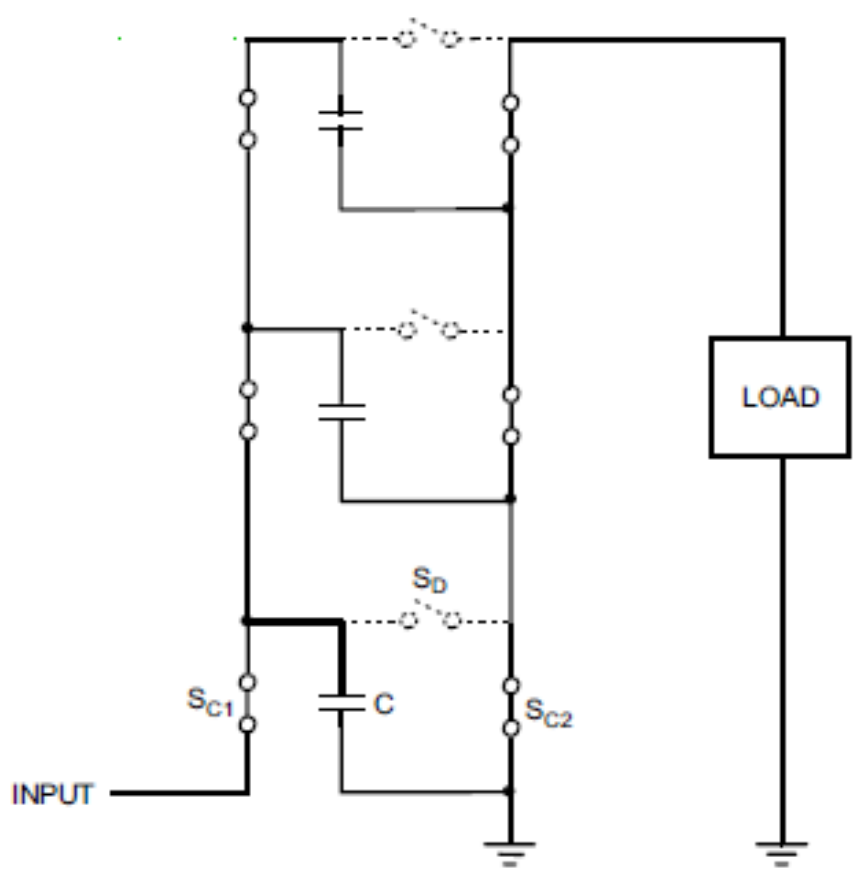

b)

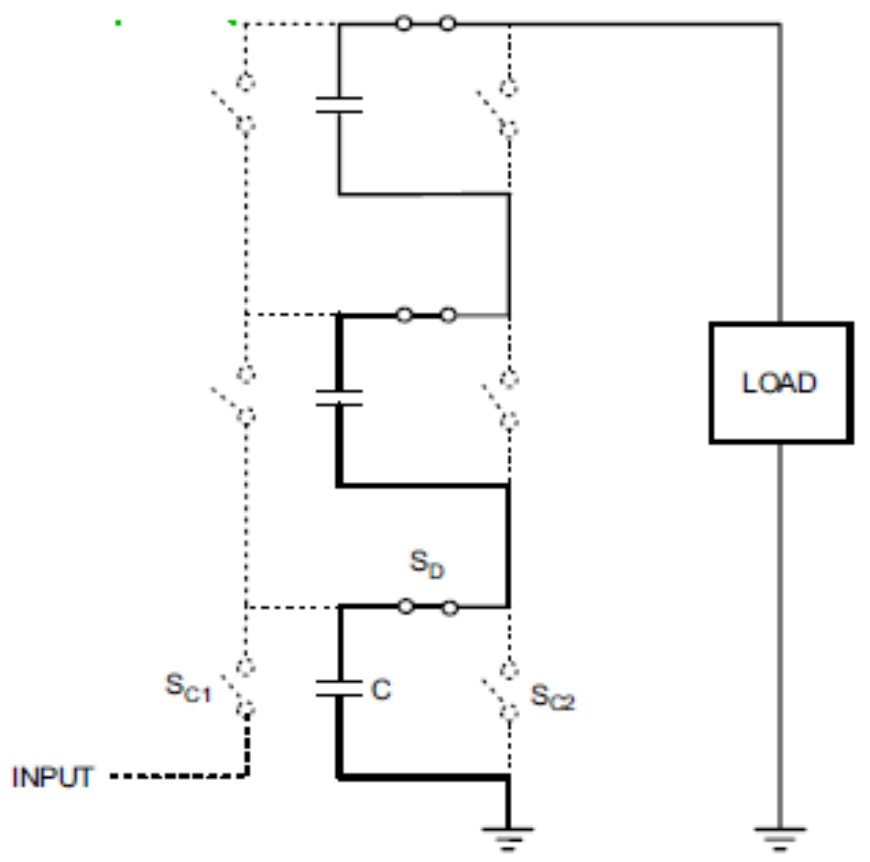

Figure 4.6: A 3-stages Marx generator layout; figure a) shows the current charging path, while figure $b$ ) gives the discharging current path. 


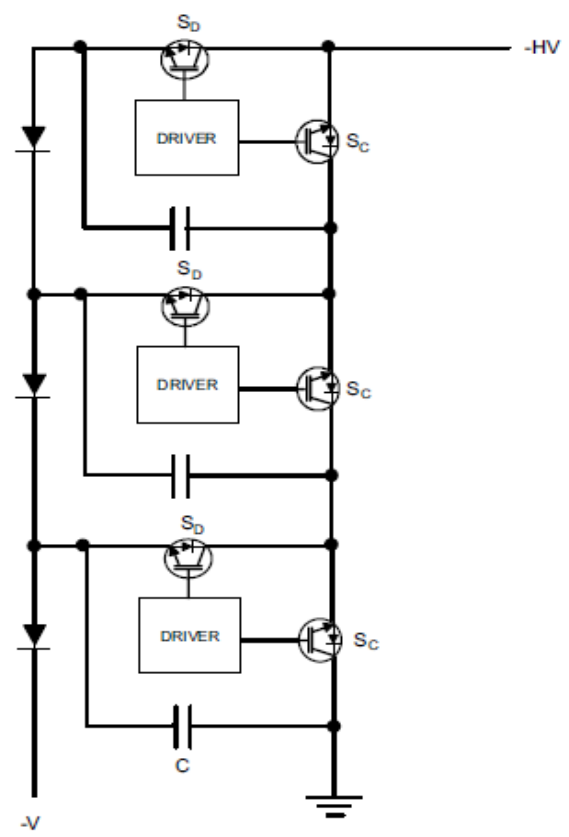

Figure 4.7: An example of 3-stages Solid State Marx Bank employing ideal charge and discharge IGBTs $\left(\mathrm{S}_{\mathrm{C}}\right.$ and $\left.\mathrm{S}_{\mathrm{D}}\right)$.

\subsubsection{Solid State Marx Generator}

In recent years, the classical Marx layout has been improved by using semiconductor switches that increase control, efficiency and pulse form [24, 25]. In a Marx design, the danger of overvoltage is also minimized because switches don't hold-off large potentials and remain isolated within the subsystems. In many cases the improvements consist solely of replacing traditional switches (e.g. spark gaps) with solid-state devices, but traditional passive elements (inductive chokes of resistors) are kept to provide charging paths and stage isolation. This is an improvement only half-complete. While newer switching technologies bring certain advantages to the fore, passive elements continue to inhibit performances through an inherent trade-off between efficiency and duty cycle. Charging resistances must be large enough to isolate stages during the discharge (force energy through the load) but small enough to allow rapid recharging in the dead time between pulses. This trade-off is the reason for why chokes are now typically used instead of resistors, yet they become themselves impractical when longer pulse widths are required. To balance the trade-off, components are selected for a specific duty cycle. A deviation from this configuration is detrimental to performance. A solid state Marx generator design is outlined in Figure 4.6 and in Figure 4.7. The first shows the general design concept where the passive elements $R_{1}$ and $R_{2}$ in Figure 4.5 have been replaced by a solid state charge and discharge switches $S_{C}$ and $S_{D}$ respectively. In order to charge the storage capacitors, all switches $S_{D}$ are opened and all 


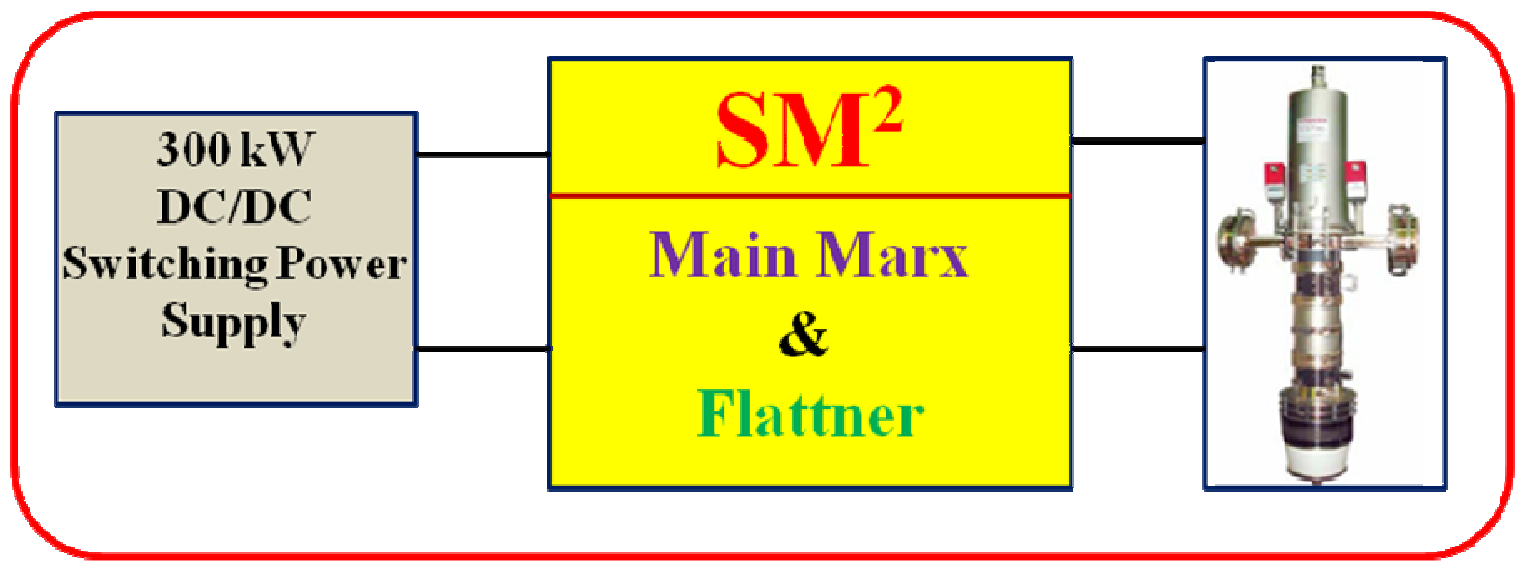

Figure 4.8: Klystron power supply complex, using our Solid State Marx Modulator ( $\left.\mathrm{SM}^{2}\right)$.

switches $\mathrm{S}_{\mathrm{C}}$ are closed. Once the capacitors are charged, the Marx bank is fired into the load, by opening switches $S_{C}$ and closing switches $S_{D}$. At the end of the high voltage pulse, their states invert once again to recharge the storage capacitors. Figure 4.3 shows how an ideal Marx bank may be realized through solid-state power components alone. In this implementation, the charging element $\mathrm{R}_{1}$, of a classical Marx modulator has been replaced by a high voltage switching diode and $\mathrm{R}_{2}$ by an IGBT-based switch. The diode has a low impedance path for charging storage capacitors in parallel, but provides high impedance isolation during their discharging in series. It also provides a bypass path when the $S_{\mathrm{D}}$ switch fails to close, during the discharge. In such a case, the system output is simply reduced and the amount of energy remains in the isolated stage. This configuration allows the stages to charge simultaneously and therefore enforces an equal share of electrical stresses at all times. Note that an active "pull-down" through the charge switches $S_{C}$ is inherent in the designs of Figure 4.6 and Figure 4.7. Such a feature rapidly discharges stray and load capacitances at the beginning of the charge cycle, to give a sharper pulse trailing edge.

\section{4 $\mathrm{SM}^{2}$ a Solid State Marx Modulator for ILC}

$\mathrm{SM}^{2}$, Solid-State Marx Modulator is a transformerless-based solid state Marx generator, developed by a group of physicist and engineers of the University of Cassino (Italy) working inside the Istituto Nazionale di Fisica Nucleare (INFN) NTA-ILC Collaboration.

A solid state Marx topology will not need to use any high voltage transformer. As matter of fact this design has several advantages over the conventional klystron modulator designs:

* it is physically smaller;

* there is no pulse transformer (quite massive for the needed voltages and currents);

* no need of large energy storage capacitor banks, owing to the active droop 


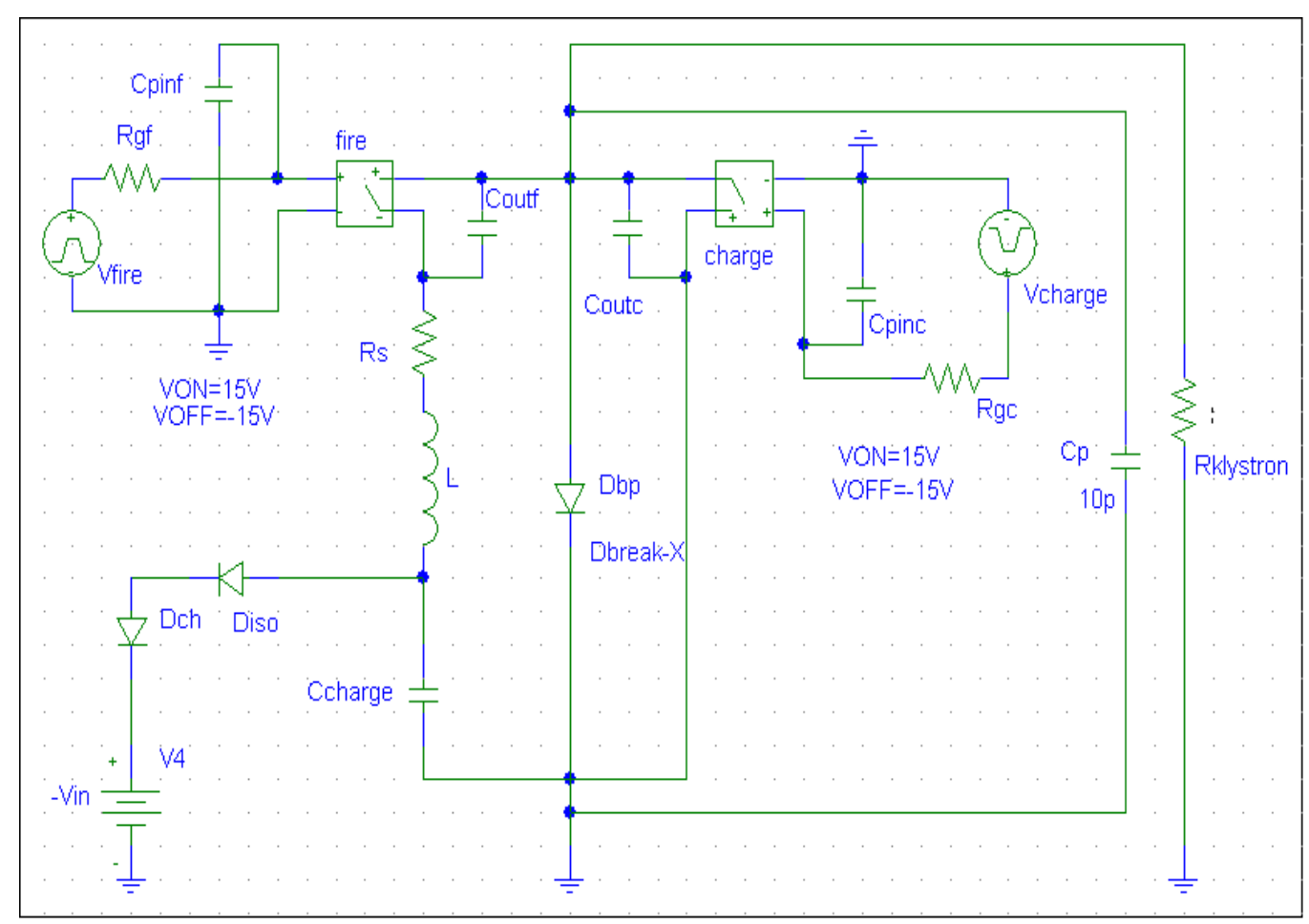

Figure 4.9: The $\mathrm{SM}^{2}$ fundamental cell layout.

compensation;

* it is oil-free;

* voltage hold-off is achieved by using air insulation;

* it is air cooled;

* secondary air-water heat exchanger is physically isolated from the electronic components.

Figure 4.8 shows a block diagram of the klystron power supply complex. In particular is shown a $300 \mathrm{~kW}$ DC/DC switching power supply which have to be capable to produce a stabilized input voltage for $\mathrm{SM}^{2}$. The Solid-State Marx Modulator, that supplies with particular requirements, already mentioned, the $10 \mathrm{MW}$ L-band klystron, is constituted by a series of two specific circuits. The first circuit, that will be called also Main Marx (MM), is a solid state switch Marx generator that uses 24 identical fundamental cells. The MM is supplied by an input voltage stabilized of $5 \mathrm{kV}$ and it produces an output pulsed signal with a width of $2 \mathrm{~ms}$, a repetition rate of $10 \mathrm{~Hz}$, an amplitude of $120 \mathrm{kV}$ with a output current of 120 A and a output signal droop of about $13 \%$. The second circuit, that will be call also Flattener, is a solid state switch smaller Marx generator that uses 19 identical fundamental cells to achieve an output drop compensation of about $1 \%$, as required by the ILC design report. The Flattener is supplied by an input stabilized voltage of $1.2 \mathrm{kV}$ and it is opportunely fired to reduce the output pulse droop. 


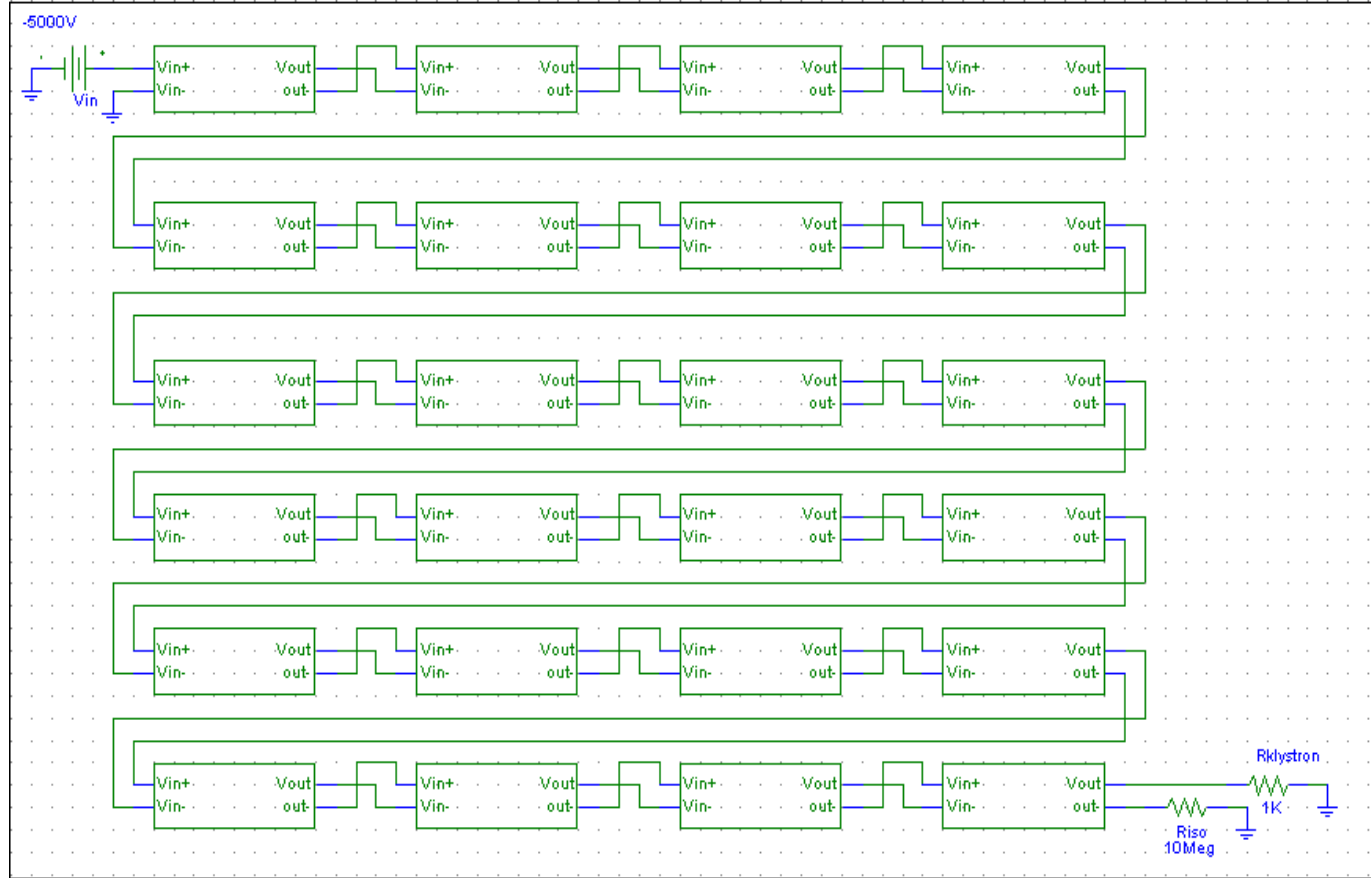

Figure 4.10: The Main Marx block layout.

\subsubsection{The $\mathrm{SM}^{2}$ fundamental cell design}

The layout of the single cell of the $\mathrm{SM}^{2}$ modulator, the fundamental cell, is shown in Figure 4.9. The key components of each fundamental cell are:

- a charge diode $\left(\mathrm{D}_{\mathrm{ch}}\right)$ that provides a path for charging the main capacitor $\left(\mathrm{C}_{\text {charge }}\right)$ to input voltage;

- an isolation diode $\left(\mathrm{D}_{\text {iso }}\right)$ that provides isolation between a single cell and the other cells during erection;

- a charge switch (charge) that provides the charging path for the main capacitor during a charging period of $100 \mathrm{~ms}$;

- a fire switch (fire) which closure will produce $\mathrm{a}-\mathrm{V}_{\text {in }}$ output pulse from the cell of a 2 ms pulse length;

- a by-pass diode $\left(\mathrm{D}_{\mathrm{bp}}\right)$ that provides a conduction path to the load through the cell or the cells that have not been fired;

- a series inductor $\mathrm{L}$ to limit $\mathrm{dI} / \mathrm{dt}$ in the output current. 


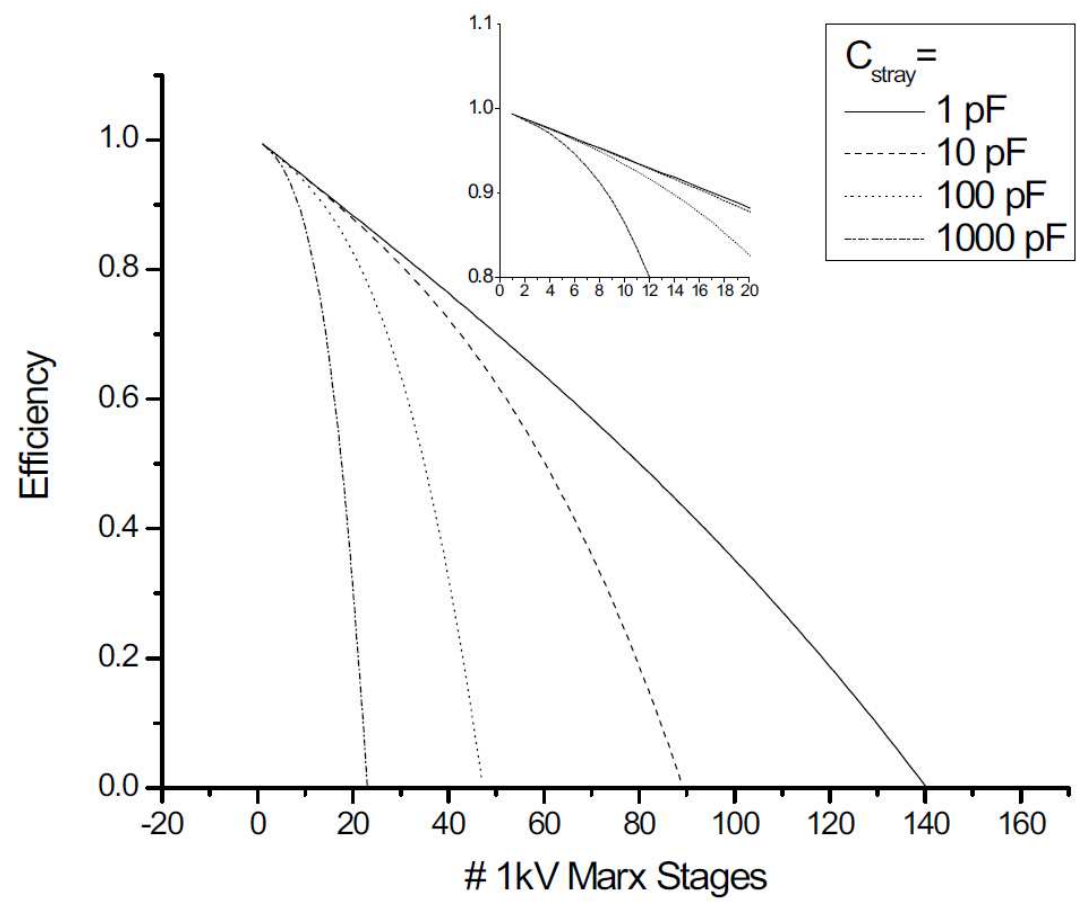

Figure 4.11: Marx bank efficiency versus the number of Marx stages for various stray stage capacitances. The inset shows a close-up of 10-stages modulator efficiency.

\subsubsection{The $\mathrm{SM}^{2}$ Main Marx Generator}

In the previous chapter, we discussed the layout of a fundamental cell of the $\mathrm{SM}^{2}$ Modulator. In this paragraph, we will describe the development of a Marx modulator able to produce output pulsed signals of $120 \mathrm{kV}$, for a current of $120 \mathrm{~A}, 2 \mathrm{~ms}$ base pulse width and a repetition rate of $10 \mathrm{~Hz}$. We will start reviewing the circuit requirements and then we will discuss the results of the simulations performed in order to study the behavior of a fundamental cell and of the full Main Marx generator.

\subsubsection{The Main Marx Layout and Design Requirements}

The Main Marx modulator is a Marx generator that uses solid state switches. MM uses 24 identical fundamental cells. The MM design requirements are the following:

- An input stabilized high voltage of $5 \mathrm{kV}$ and an high output voltage of $120 \mathrm{kV}$;

- An output voltage droop of about $13 \%$;

- An output current of $120 \mathrm{~A}$; 


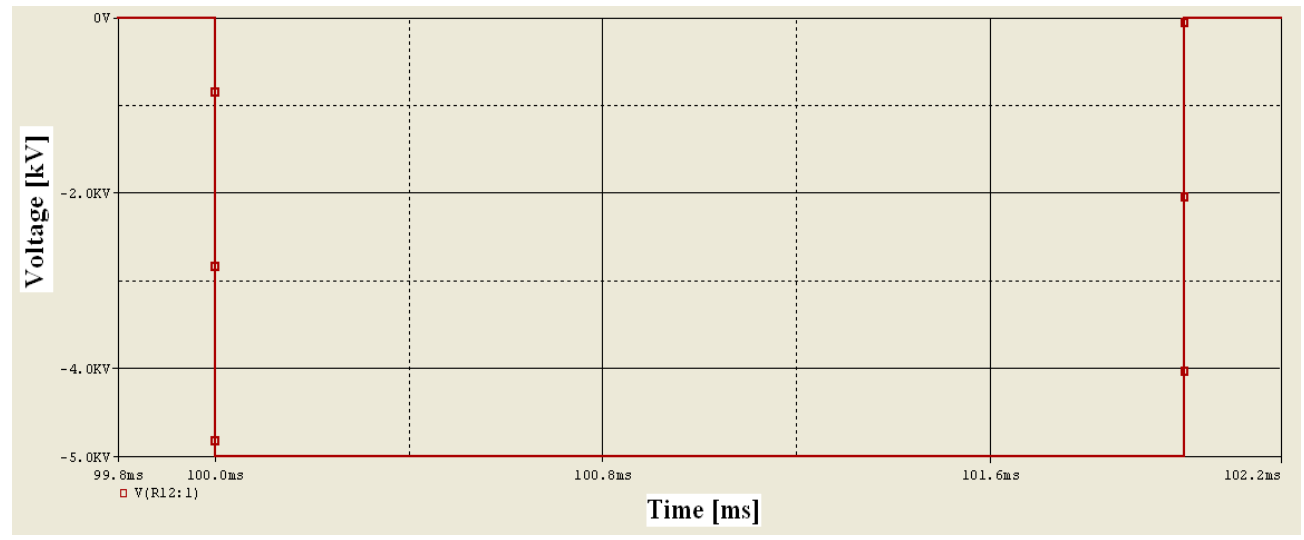

Figure 4.12: Simulated single cell output voltage.

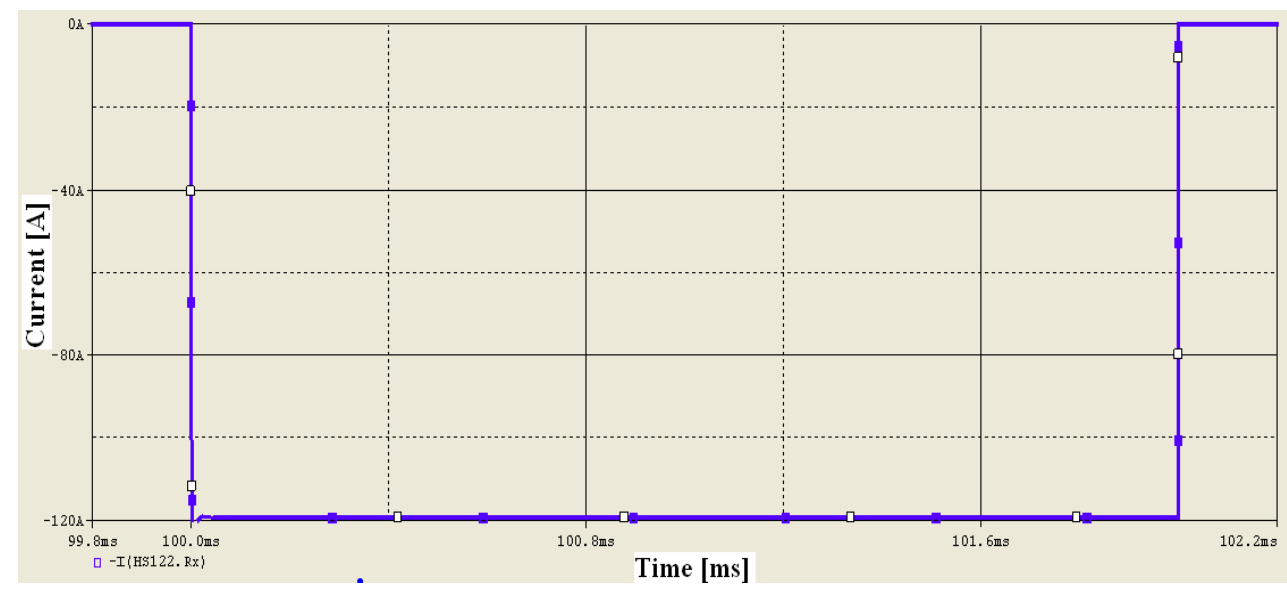

Figure 4.13: Simulated single cell output current.

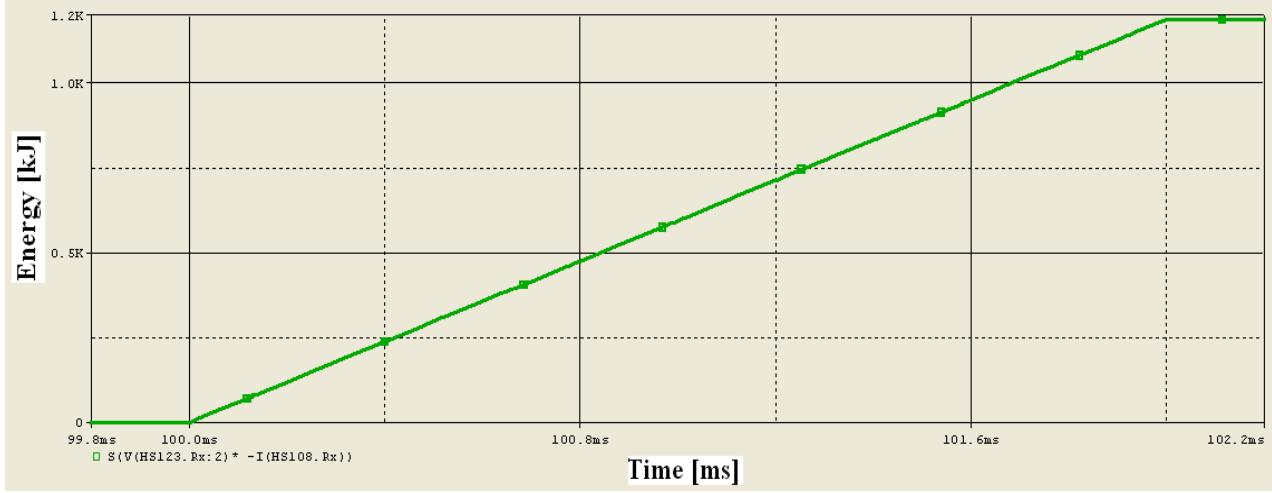

Figure 4.14: Simulated single cell output energy. 
- The main capacitors must charge in parallel in $100 \mathrm{~ms}$ at input voltage level;

- The main capacitors must discharge in series to produce an output pulse of $2 \mathrm{~ms}$ base pulse width;

Because of these requirements, we end up to have a MM circuit with a number of cell equal to 24 and a Main Capacitor, for storing the circuit energy, of $350 \mu \mathrm{F}\left(\mathrm{C}_{\text {charge }}\right.$ in Figure 4.9). The cells have been designed also in an way that each of them accumulate an energy below the value of $1.18 \mathrm{~kJ}$. As matter of fact, this energy value is below to the maximum energy that can be reversed to the klystron without risking to damaging it.

\subsubsection{Stray Capacitance Charging Losses}

Some of the Marx-stored energy is required to repetitively charge stray capacitances that might be present in the bus work of a modulator. This energy cannot be recycled and is lost during the active pull-down. The higher modulator resides on the potential ladder, the more energy is required to charge inherent stray capacitances $C_{P}$ per stage with respect to ground. So the total power lost to this process, assuming stray capacitances are well balanced is,

$$
P_{\text {stray }}=\frac{f}{2} \sum_{k=1}^{N} C_{p}\left(k V_{\text {in }}\right)^{2}
$$

Inefficiencies relating to stray capacitance increase quadratically with the number of stages $\mathrm{N}$ and as such impose an upper practical limit on the number of stages in a Marx modulator. In order to minimize stray capacitances, design efforts must reduce the physical size of the modulator footprint, components and stage interconnects. Curves of efficiencies for various values of stray capacitance are plotted versus number of stages in Figure 4.11. From this graph it is apparent that at $10 \mathrm{kV}$ the efficiency remains above $90 \%$, even with liberal amounts of internal stray capacitances. In the $\mathrm{SM}^{2}$ cell layout (Figure 4.9) the value of stray capacitance was assumed equal to $10 \mathrm{pF}$.

\subsubsection{Main Marx output response}

The circuit simulations have been implemented by using the schematic session of the OrCad 9.2 program. OrCad is a general-purpose circuit simulation for non-linear DC, non-linear transient and linear AC analyses fully owned by Cadence Design Systems. We have performed a transient analysis to computes the transient output variables as a function of time over a user-specified time interval. The initial conditions were automatically determined by a DC analysis that determines the DC operating point of the circuit with inductors shorted and capacitors opened. The main circuit elements and simulation parameters used are: 


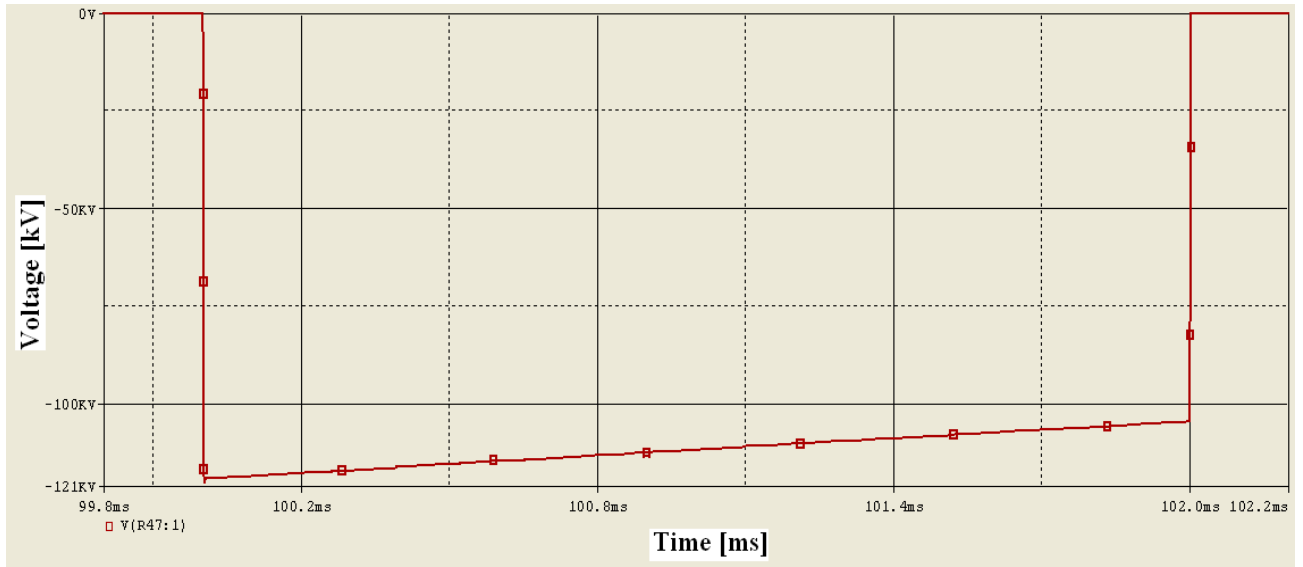

Figure 4.15: Simulated Main Marx output voltage; the droop in the output voltage has a value of about $13 \%$.

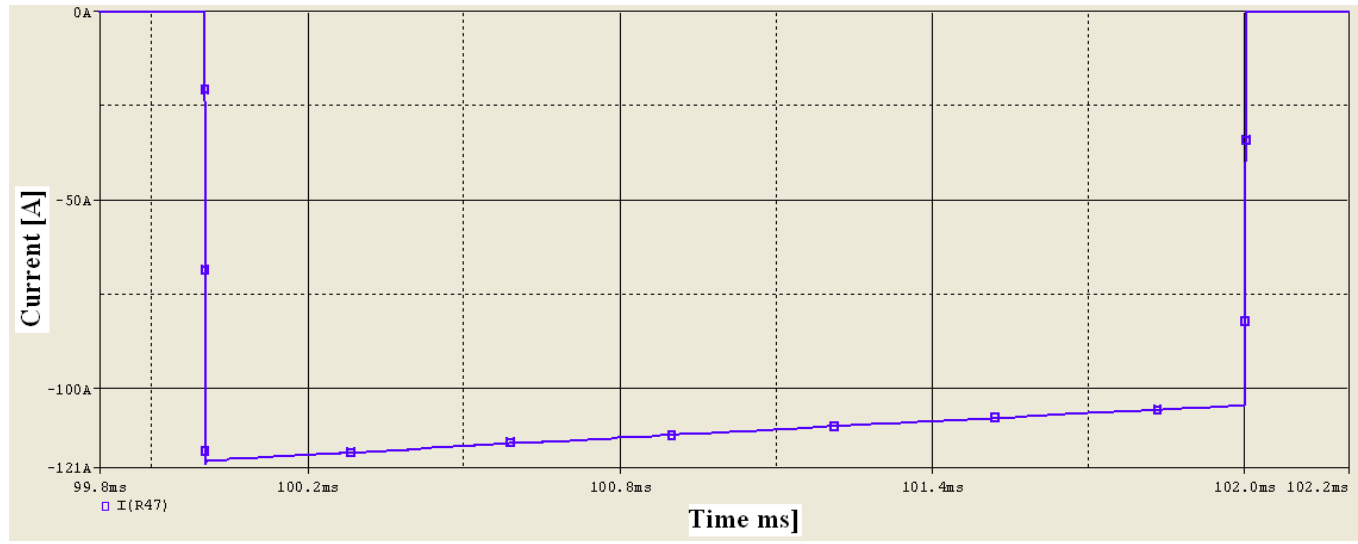

Figure 4.16: Simulated Main Marx output current; the droop in the output current has a value of about $13 \%$.

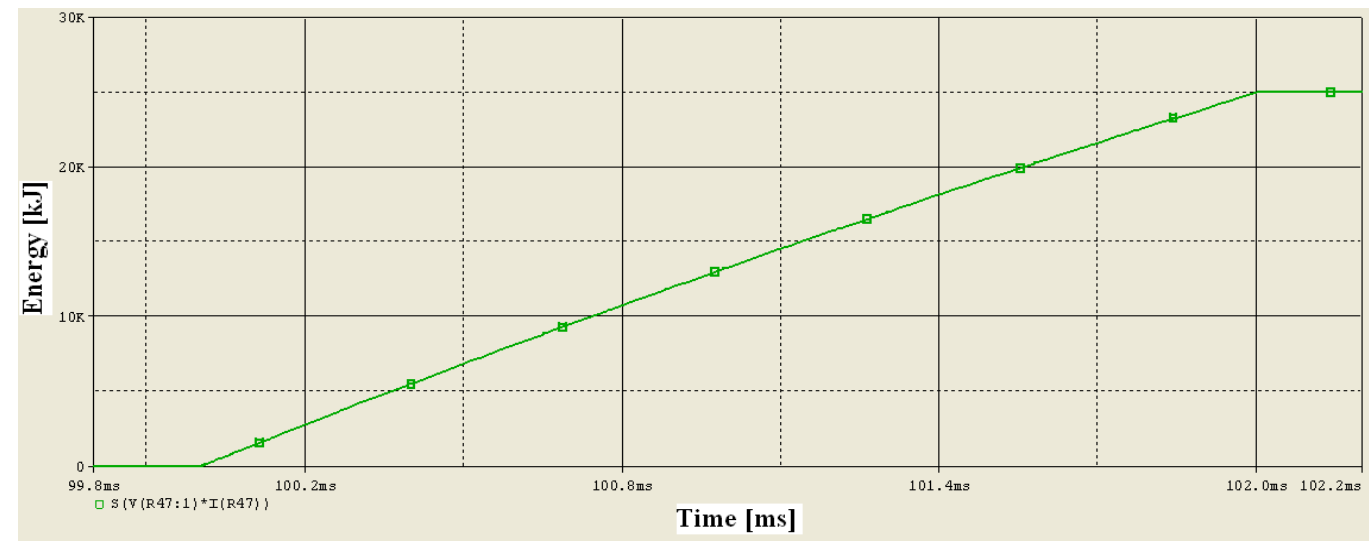

Figure 4.17: Simulated Main Marx output energy. 
- Linear resistors, capacitors and inductors;

- Stray resistance and capacitance;

- Real power diode and real power IGBTs;

- DC voltage sources and independent sources as pulse sources to switches control;

- A transient period of $50 \mathrm{~ms}$;

Also the use of an ideal element that is highly non-linear such as a switch can cause large discontinuities to occur in the circuit node voltage. A rapid change such as that associated with a switch changing state can cause numerical round-off or tolerance problems leading to erroneous results or time-step difficulties. So to improve this situation was used:

- A step ceiling of $10^{-3}$;

- A relative tolerance of $10^{-2}$

In order to perform a realistic study of the circuit behavior, we used, in the simulation, real circuital elements by implementing real switches and by adding, to each circuital component, parasitic capacitance and resistance as well as real tolerances. These quantities have been also used, later, in the Montecarlo simulations of the full $\mathrm{SM}^{2}$ Modulator behavior under different operational conditions.

The output response of our simulations, performed on a single fundamental MM cell, is given in Figure 4.12, 4.13 and 4.14, where the output voltage, the output current and the output energy are plotted. Figure 4.15, 4.16 and 4.17, on the other hand, show the behavior of the full Main Marx generator. As it is possible to see, the droop in the output voltage and in the output current is around $13 \%$ of the total voltage and current value $(120 \mathrm{kV}, 120 \mathrm{~A})$.

\subsubsection{The Flattener circuit}

In the $\mathrm{SM}^{2}$ Modulator, the droop regulation of the output pulses up to a value of $\pm 0.5 \%$ is achieved by adding a small Marx in series with the Main Marx. This small Marx generator, the Flattener circuit, is composed of 19 fundamental $1.2 \mathrm{kV}$ cells similar to the one used for the Main Marx. The flattener cells are then fired sequentially in order to be able to generate a series of discrete corrections on the Main Marx output signal droop. If we compare this droop compensation strategy to the droop compensation achieved in a bouncer-like correction, the conclusions are that our scheme is much smaller and less expensive than the other. Figure 4.18 shows the overall modulator, characterized by the Flattener in series with the Main Marx. The time regulation of such a system is very crucial in order to achieve the droop compensation up to the design requirements. In the next paragraph, we will describe how the Flattener charging and discharging switches are fired and in particular we will show the control driving law that must be implemented, together with the trigger system, in order to obtain a good performance of Flattener circuit. 


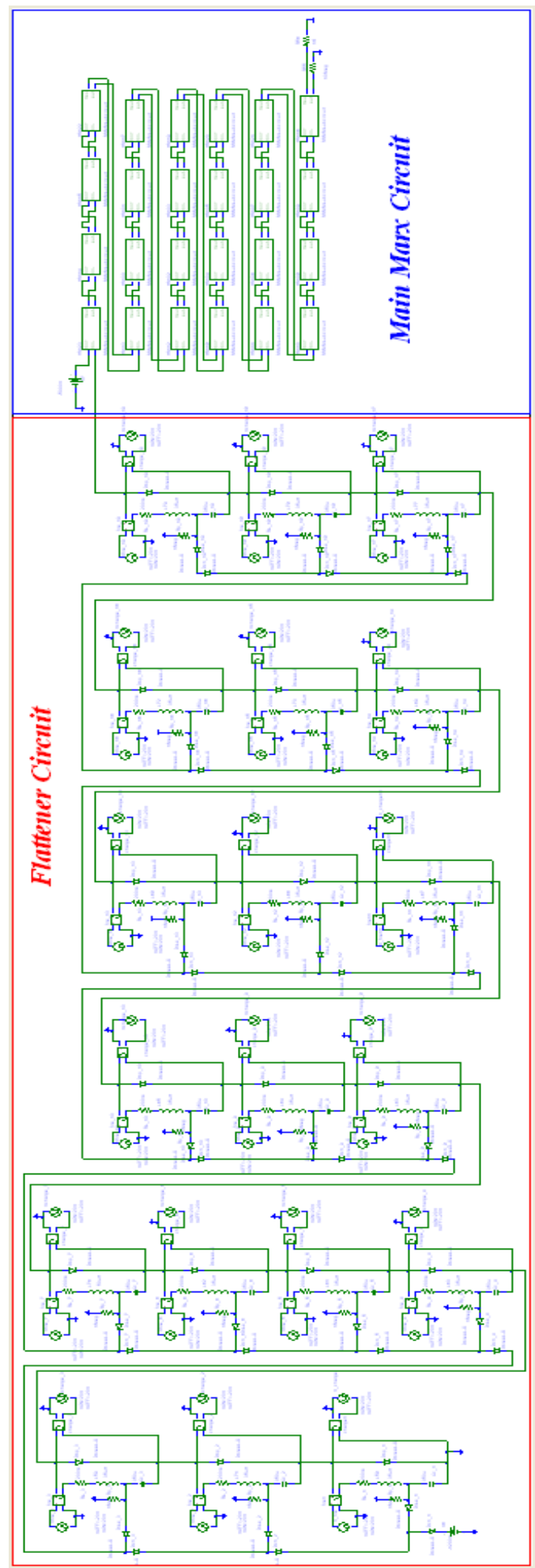

Figure 4.18: Overall $\mathrm{SM}^{2}$ Marx Modulator layout. As it is possible to see in the circuit schematic, the Flattener circuit is in series with the Main Marx generator. 


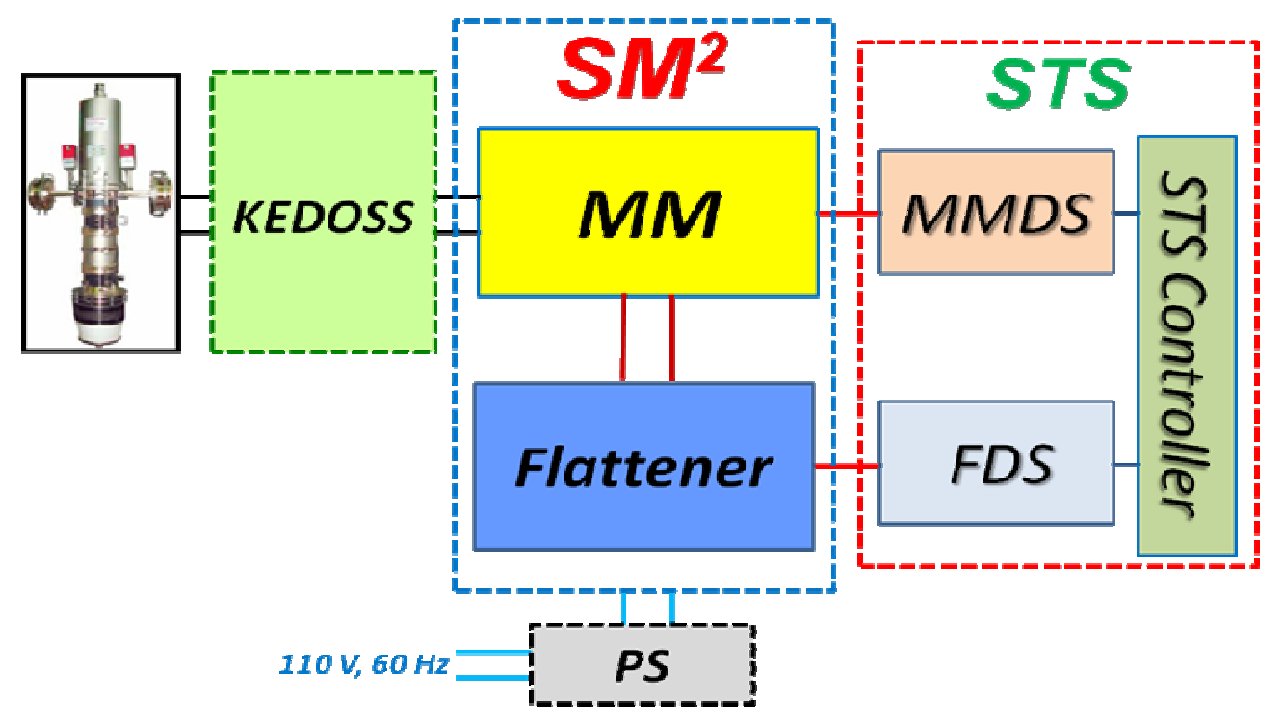

Figure 4.19: Full $\mathrm{SM}^{2}$ Modulator conceptual design, where STS is the Switching Trigger System for the $\mathrm{SM}^{2}$ modulator, $\mathrm{MM}$ is the $\mathrm{SM}^{2}$ Main Marx modulator, the MMDS is the Main Marx Driving System, FDS is the Flattener Driving System and the KEDOSS is the Klystron Energy Droop Out Safety System. $\mathrm{PS}$ is the $\mathrm{SM}^{2} \mathrm{DC} / \mathrm{DC}$ switching power supply.

\subsubsection{The $\mathrm{SM}^{2}$ Switching Trigger System}

The implementation of the $\mathrm{SM}^{2}$ Switch Trigger System (STS) is an important part of our solid state Marx generator complex. It is mainly based on a general controller that acts on two different digital trigger systems:

- The Main Marx Driving System (MMDS) that is used to generate the control signal sequence, for the Main Marx Generator switch charging and discharging. The MMDS controls the closing of all charging switches during a period time of $100 \mathrm{~ms}$, to allow the main capacitor charging $\left(\mathrm{C}_{\text {charge }}\right)$ in parallel and the closing of all the discharging switches during the output signal creation in a period of time of $2 \mathrm{~ms}$.

- the Flattener Driving System (FDS) that is used in order to fire, with an appropriate time control sequence, the charging and discharging switches of the Flattener circuit in order to open and close sequentially the switches for the Main Marx droop compensation in the high voltage output pulses.

It has to be noticed that the MMDS and the FDS must be accurate in order to fire simultaneously all charging switches during the charging time and all discharging switches during discharging period. Nowadays, the implementation of such an apparatus can be easily achieved by using Field-Programmable Gate Array devices (FPGA) and optical fibers for trigger signal transport. Figure 4.19 gives a schematic overview of the full Solid State Marx 


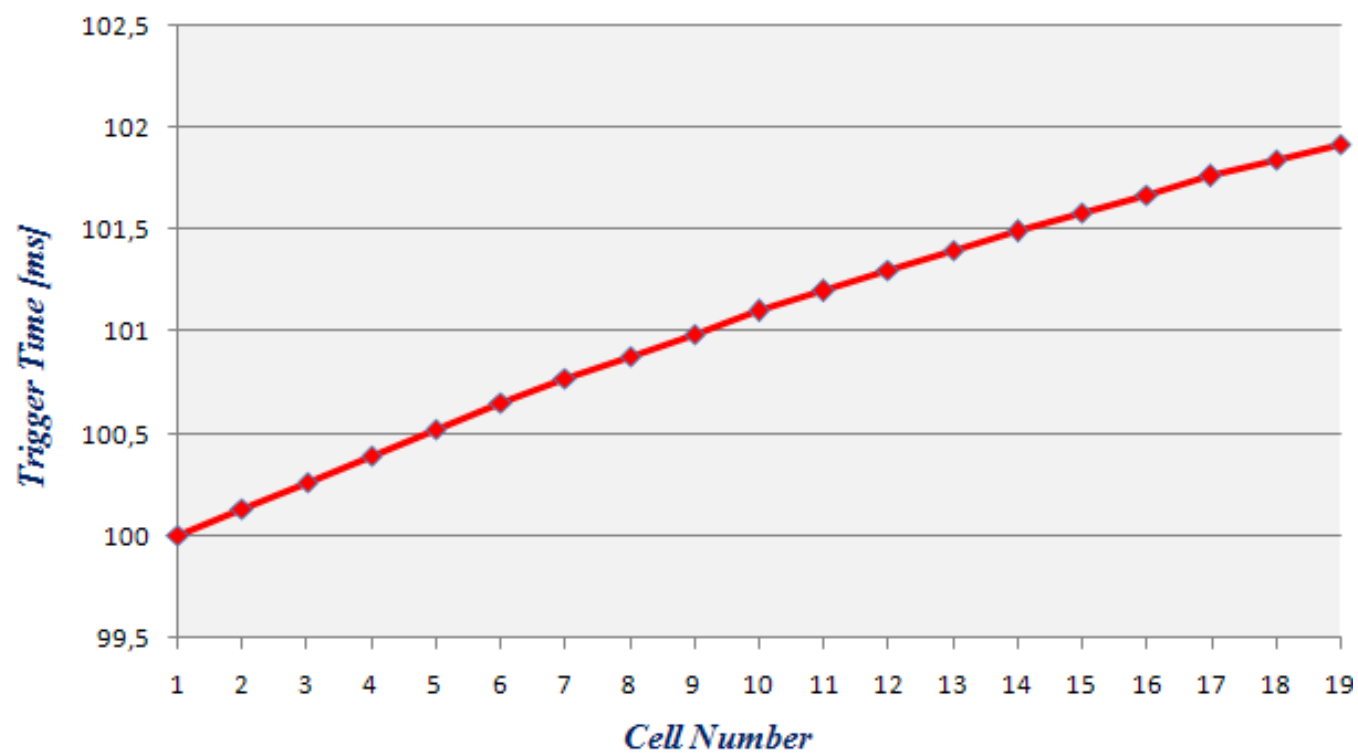

Figure 4.20: Sequence of the trigger signals necessary to operate the Flattener charging switches.

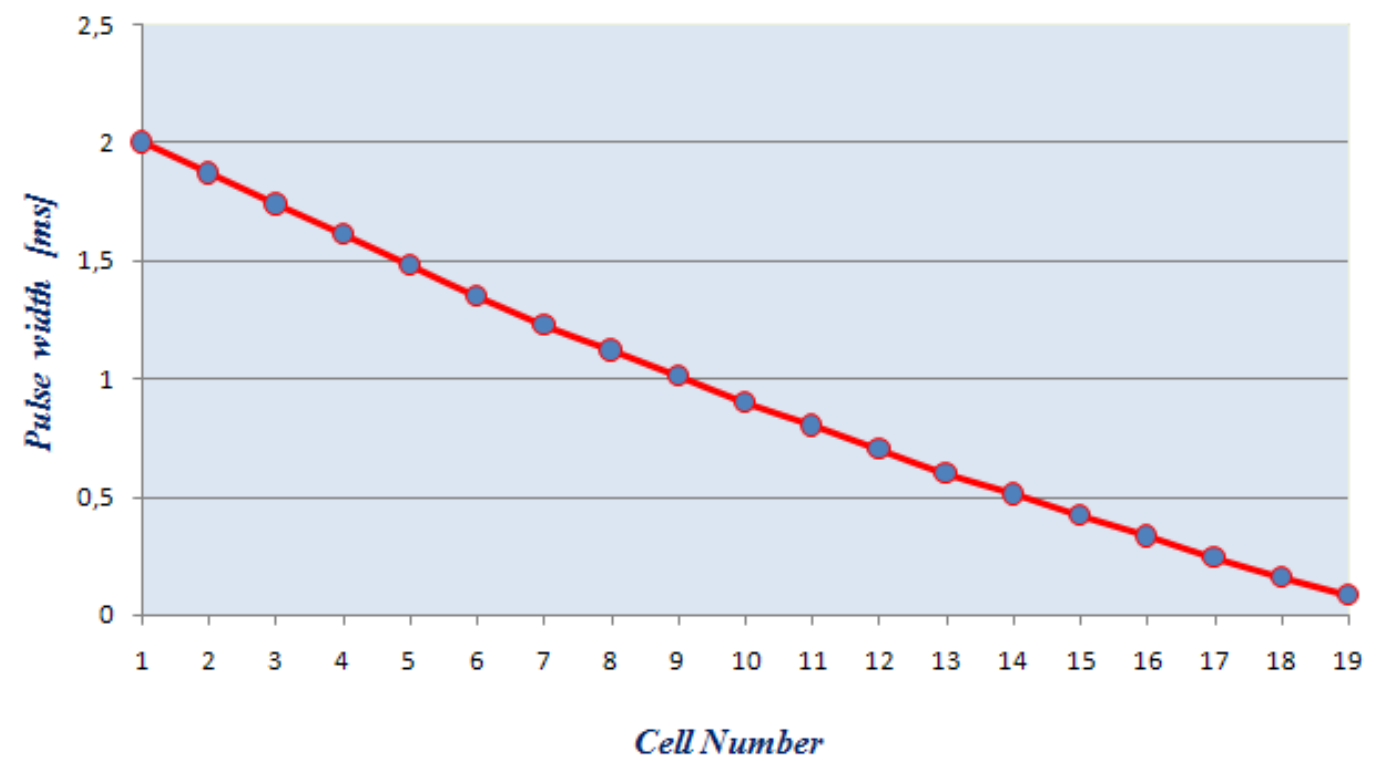

Figure 4.21: Pulse width of the trigger signals necessary to operate the Flattener discharging switches. 


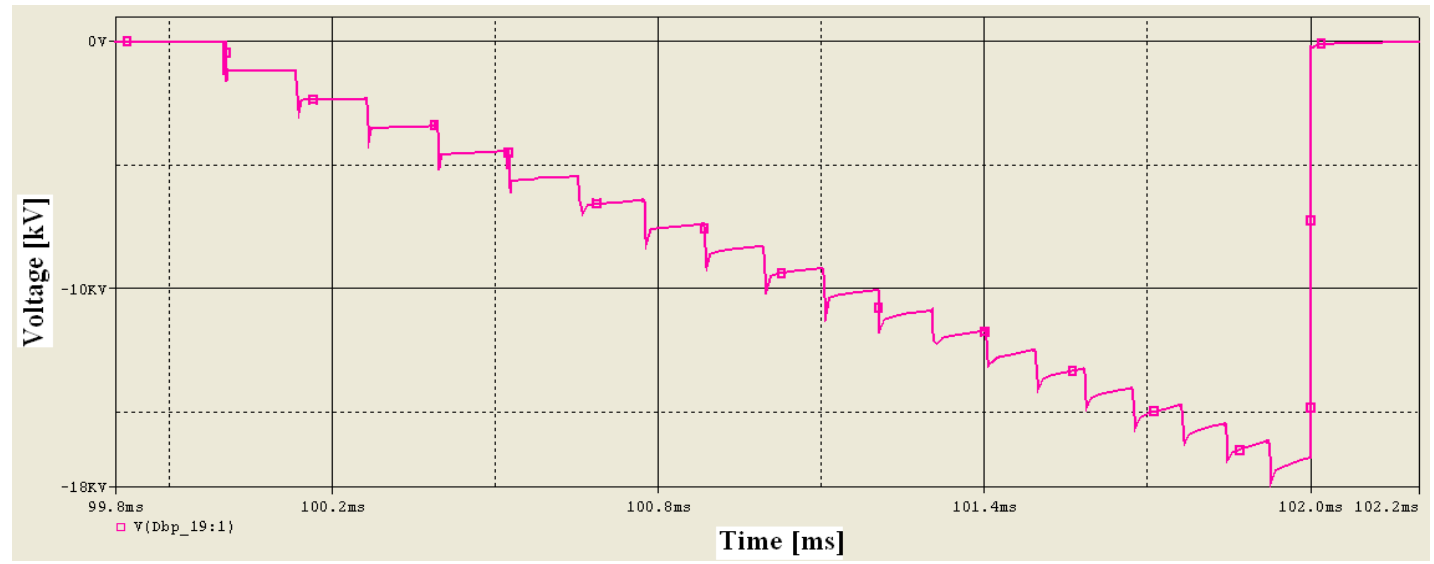

Figure 4.22: Simulated Flattener output voltage.

Modulator and Switching Trigger System. In Figure 4.20, 4.21 we report the time sequence needed to trigger the charging and discharging switches of the Flattener. In particular, Figure 4.20 shows the sequence of the trigger signals necessary to operate the Flattener charging switches, while in Figure 4.21 the pulse widths of the trigger signals, necessary to operate the Flattener discharging switches, are illustrated. Finally, the simulated output signal coming from the Flattener circuit is given in Figure 4.22.

\subsection{Solid State Marx Modulator single Failure Risks}

The probability of critical component failure increases directly with the number of elements. In the basic design scheme, the failure of any single IGBT or capacitor will fail the entire modulator by shorting out the input DC power. These critical single-point failures must be eliminated in any practical design by using a large numbers of cells with a lower ripple voltage. The transmission scheme, for vital control data and timing fiducials, must also be massively parallel in nature, to avoid single-point control-induced failures. Failures of any IGBT or capacitor, in a basic Solid State Marx Modulator design, will fail the entire modulator. These single-point risks can be mitigated by adding a simplified high-side IGBT buck regulator to each cell. This regulator serves three vital functions:

1. Control of inrush currents during the charging cycle;

2. Local regulation of the cell operating voltage;

3. Isolation of the cell from the main DC charging path, in the event of a cell failure.

Another important characteristic of any klystron modulator have the protection from arcs, when the klystron faults. The klystron is a very expensive device and it will probably arc at some time of its life. Regarding the ILC TDR klystron model: Toshiba MBK 3736, that works typically with the following voltages and currents $\mathrm{V}_{\mathrm{In}}=120 \mathrm{kV}, \mathrm{I}_{\mathrm{In}}=120 \mathrm{~A}$, 

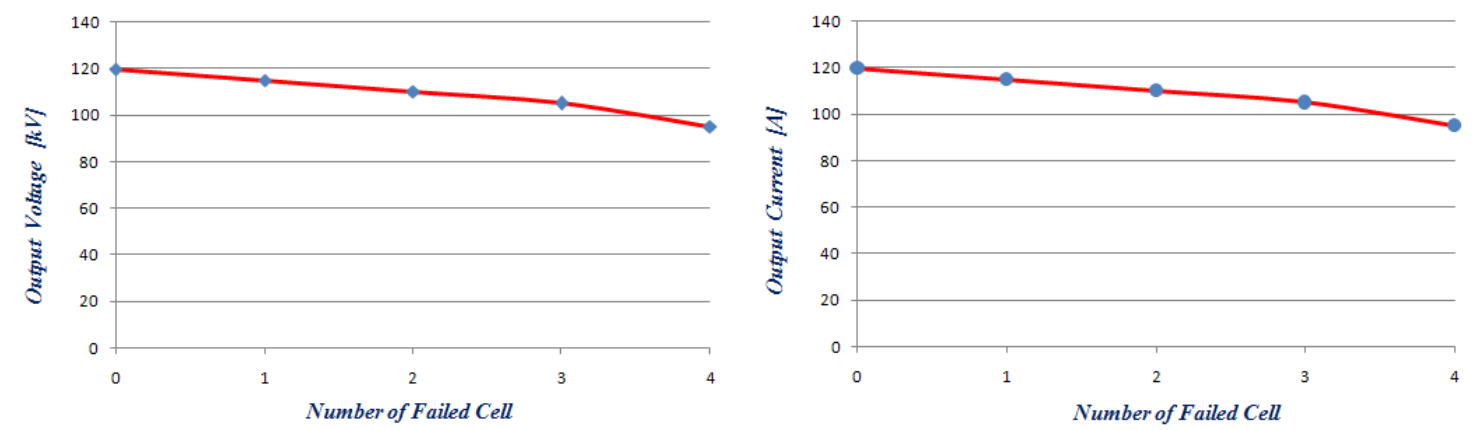

Figure 4.23: Simulated voltage (a) and current (b) droop as a function of number of cell that failed in the $\mathrm{SM}^{2}$ modulator.

$\left(\mathrm{R}_{\mathrm{Load}}=1 \mathrm{k} \Omega\right)$ the $\mathrm{SM}^{2}$ modulator is expected to deliver an energy to the klystron of about $\mathrm{E}=$ $\mathrm{RI}^{2} \mathrm{~T}=28.8 \mathrm{~kJ}$. Then, an arc protection system has to be implemented. Figure 4.21 shows the $\mathrm{SM}^{2}$ modulator conceptual design where the Klystron Energy Drop-out Safety System (KEDOSS) is also represented. The development of the KEDOSS apparatus goes beyond the goals of the present work. Anyhow there are several solutions under consideration. The first one that we considered is a conventional crowbar circuit that suddenly quenches the klystron arc into a discharge of the stored energy. However the crowbar circuit has false-firing problems caused by electric noise and other crowbarless solutions have been considered. In Figure 4.23.a and in Figure 4.23.b we show the simulated current and voltage droop as a function of the number of cell that failed inside the $\mathrm{SM}^{2}$ Modulator. The behavior reflects what expected as Marx generator adds, to each step, linearly the same voltage step-up: $V_{\text {out }}=$ $\mathrm{N} \cdot \mathrm{V}_{\text {cell }}$. As the probability of $\mathrm{N}$ independent failures inside $\mathrm{SM}^{2}$ goes as $\mathrm{P}^{\mathrm{N}}$ this plot show that a failure of a single cell doesn't quench the modulator output response.

\subsection{The $\mathrm{SM}^{2}$ Marx Modulator circuit simulation and analysis}

In this paragraph we describe a part of the ample analysis that has been performed in order to study the behavior of our Solid State Marx Modulator design. To begin, we will show that the ILC TDR design requirements have been achieved. Figure 4.24 shows the simulated $\mathrm{SM}^{2} 120$ $\mathrm{kV}$ output train pulses; as it possible to see the repetition rate fits the design of a $10 \mathrm{~Hz}$ repetition rate modulator. Figure 4.25 shows the simulated $\mathrm{SM}^{2}$ output voltage that goes to power the klystron. This distribution has a maximum absolute value of $120 \mathrm{kV}$, a pulse width of $2 \mathrm{~ms}$ and the output voltage droop stay below the nominal TDR request of about $1 \%$ [31]. Figure 4.26 shows the simulated $\mathrm{SM}^{2}$ output current. The plot has a maximum value of 120 A, a pulse width of $2 \mathrm{~ms}$ and an output current droop that stays below the nominal TDR request of about $1 \%$. Besides, Figure 4.27 shows the $\mathrm{SM}^{2}$ produced output energy that supplies the klystron of the needed energy to power the 26 niobium superconducting cavities. 


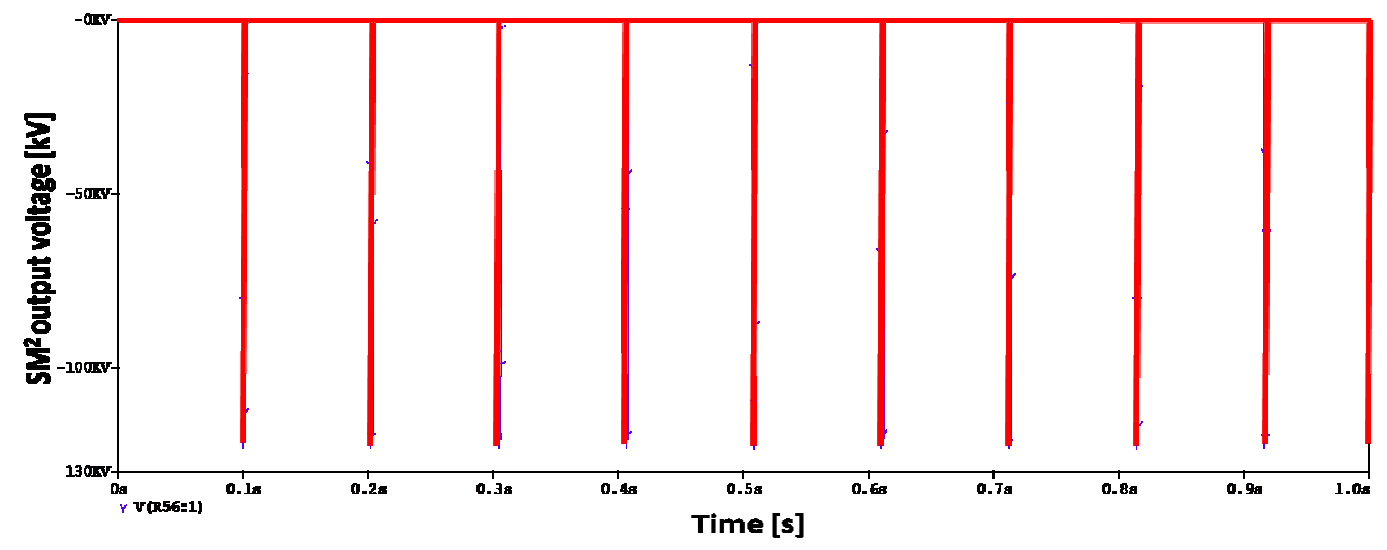

Figure 4.24: Simulated $\mathrm{SM}^{2} 120 \mathrm{kV}$ output train pulses; the repetition rate is $10 \mathrm{~Hz}$.

As already mentioned before, the klystron needs to be powered with an energy, per cycle (2 $\mathrm{ms})$, of about $27 \mathrm{~kJ}$.

As it was mention in the previous paragraphs, we implemented a realistic simulation of our Marx generator concept by accounting real parasitic resistances and inductances for every single component in the $\mathrm{SM}^{2}$ modulator and by using real IGBT based switches. In order to get a more realistic output response we had the need to perform a Montecarlo simulation. Montecarlo simulation is a method of simulation with unknown variables. In Montecarlo simulation values for unknowns are randomly selected according to their statistical distribution. The process is repeated for a number of pseudo-experiments each with a new set of values for the unknowns. The distribution of the final results is taken to be representative of the "real" behavior over the range of inputs. The Montecarlo simulation method is a way of dealing with the large number of correlated and anti-correlated and uncorrelated variables involved in circuit design. Process parameters have been characterized as a distribution of single circuital object behaviors giving the designer a large amount of data to deal with. Montecarlo simulation allows all of these variables to be considered during simulation. The Montecarlo simulation has then been performed in order to predict our circuit yield and sensitivity. The result of the Montecarlo simulation, that required and intensive CPU consuming parallel calculations, proofed that the proposed Solid State Marx Modulator design is very much stable and fits all the ILC TDR design requirements.

This very encouraging result was expected and already predicted. As matter of fact, we evaluated, following another statistical approach, which could be the worst possible scenario (probable in less than a part on $10^{8}$ ) by setting all the real components values in the worst $+1 \sigma$ and $-1 \sigma$ edge. Before performing this analysis we first verify that the quantities were not correlated or anti-correlated, and then we implemented a new set of simulations. These simulations were carried out by assuming a coherent deviation of $+1 \sigma$ and $-1 \sigma$, from the nominal values of each single real circuital element. As matter of fact we each circuital element was implemented considering the full parasitic capacitance and resistance and 


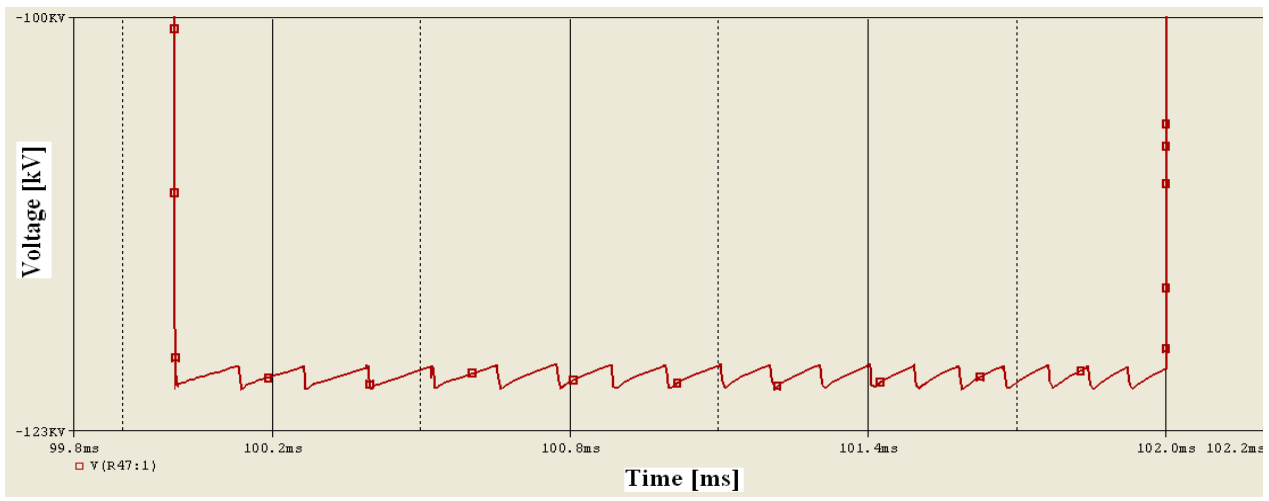

Figure 4.25: Simulated $\mathrm{SM}^{2}$ output voltage with droop compensation; the voltage droop is about $1 \%$.

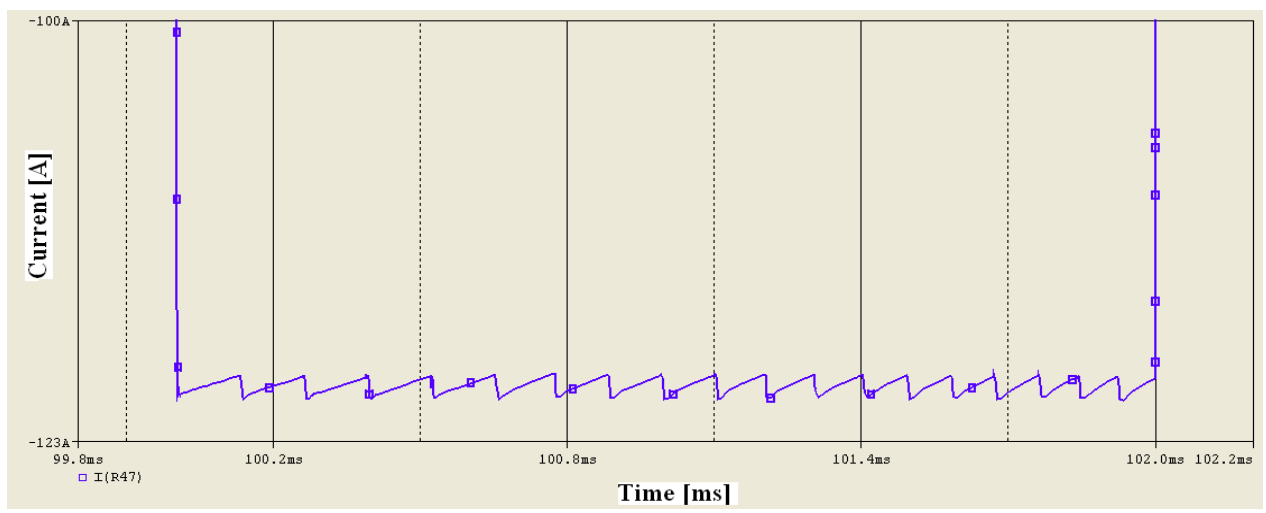

Figure 4.26: Simulated $\mathrm{SM}^{2}$ output current with droop compensation; the current droop is about $1 \%$.

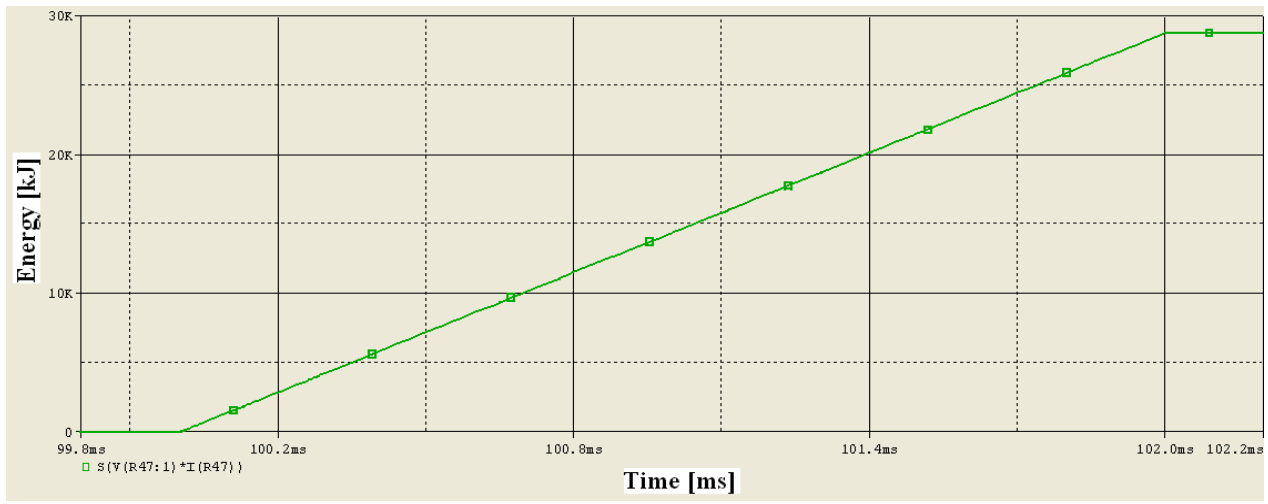

Figure 4.27: Simulated $\mathrm{SM}^{2}$ output energy. 


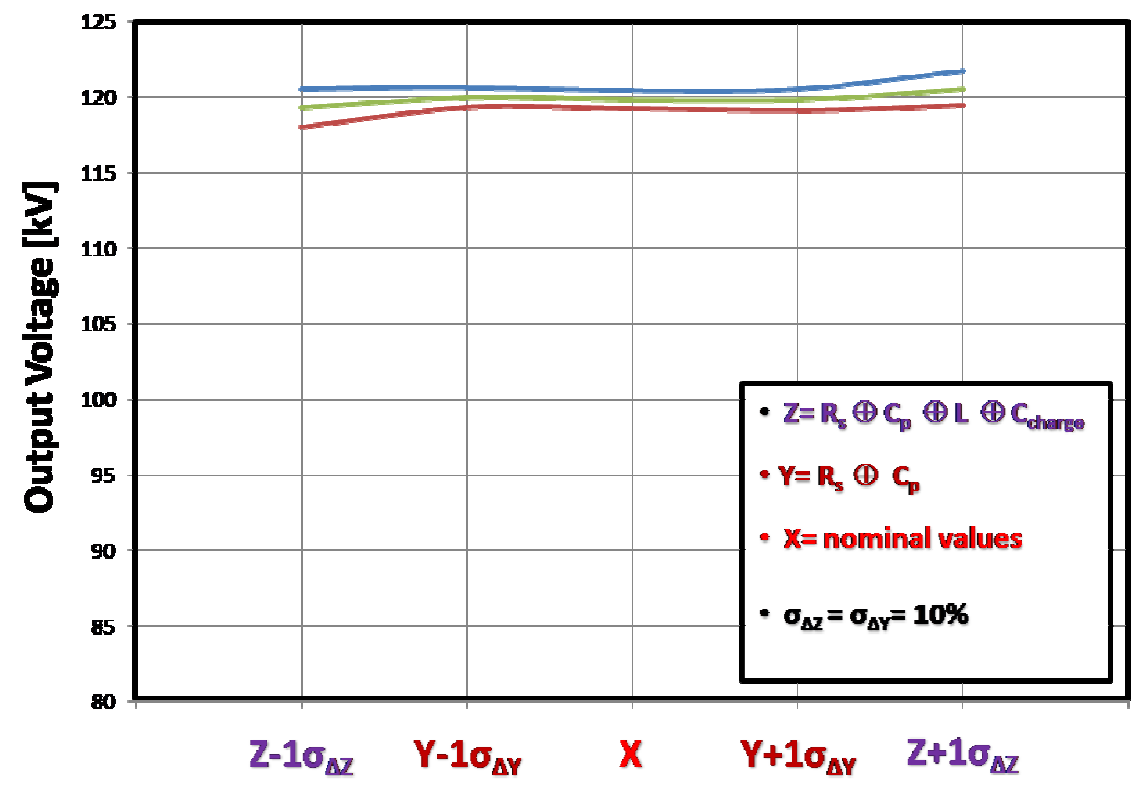

Figure 4.28: Simulated result on the output voltage, in the worst statistical scenario.

tolerances then in our simulation we had $24 \mathrm{C}_{\text {charge }}$ main capacitors, $24 \mathrm{~L}$ inductors and about 150 parasitic elements spread Marx bank that we let to fluctuate of $\pm 1 \sigma$ around the single electric component nominal tolerance. Figure 4.28 shows the result of this analysis. In the plot 4.28, X represents the result on the output voltage simulation obtained by assuming the nominal values for all the electric components involved in the $\mathrm{SM}^{2}$ Marx modulator. $\mathrm{Y} \pm$ $1 \sigma_{\Delta \mathrm{Y}}$ is then what we get by making all the parasitic elements, involved in the $\mathrm{SM}^{2}$ circuit, fluctuating of $\pm 1 \sigma$ and finally $\mathrm{Z} \pm 1 \sigma_{\Delta \mathrm{Z}}$ represent is what we obtained but assuming, at the same time, a fluctuation of the 24 Inductor values $\mathrm{L}$, of the $24 \mathrm{C}_{\text {charge }}$ main capacitors and of the 150 parasitic elements spread in our modulator. Even in the worst possible scenario the simulations shows that the $\mathrm{SM}^{2}$ functionality is very stable and pretty much independent by the component tolerances, if values around $10 \%$ are assumed.

\subsection{Conclusions}

The development of the $\mathrm{SM}^{2}$ klystron modulator topology, for the International Linear Collider (ILC), it is under development. $\mathrm{SM}^{2}$ envisioned as a smaller, lower cost, and higher reliability alternative to the present, Bouncer Modulator, now under test at Fermi National Accelerator Laboratory (Fermilab) near to Batavia, Illinois (USA). The Solid State Marx Modulator presents several advantages over conventional klystron modulator designs. It is physically smaller; there is no pulse transformer (quite massive for the ILC parameters) and the energy storage capacitor bank is quite small, owing to the active droop compensation. It is oil-free; voltage hold-off is achieved using air insulation and it is air cooled. The secondary 
air-water heat exchanger is physically isolated from the electronic components. The $\mathrm{SM}^{2}$ employs all solid state elements: IGBT based switches and diodes, to control the charge, discharge and isolation of the cells. A general overview of the $\mathrm{SM}^{2}$ Marx Modulator design and relative simulations have been presented in this work. The $\mathrm{SM}^{2}$ solution is a Solid State Marx Modulator based on double switch topology to perform the energy loading and the energy commutation to the klystron. This solution is based on four baseline elements. The first, the Main Marx (MM), that is a solid state switch Marx generator that uses 24 identical fundamental cells. The MM is supplied by an input voltage stabilized of $5 \mathrm{kV}$ and it produces an output pulsed signal with a width of $2 \mathrm{~ms}$, a repetition rate of $10 \mathrm{~Hz}$, an amplitude of 120 $\mathrm{kV}$ with a output current of $120 \mathrm{~A}$ and a output signal droop of about $13 \%$. A second part, the Flattener, that is a solid state switch smaller Marx generator that uses 19 identical fundamental cells to achieve an output droop compensation of about $1 \%$, as required by the ILC design report. The Flattener is supplied by an input stabilized voltage of $1.2 \mathrm{kV}$ and it is opportunely fired to reduce the output pulse droop. The third fundamental component of our modulator design is the $\mathrm{SM}^{2}$ Switch Trigger System (STS) mainly based on a general controller that acts on two different digital trigger systems:

- The Main Marx Driving System (MMDS) that is used to generate the control signal sequence, for the Main Marx Generator switch charging and discharging. The MMDS controls the closing of all charging switches during a period time of $100 \mathrm{~ms}$, to allow the main capacitor charging $\left(\mathrm{C}_{\text {charge }}\right)$ in parallel and then thee closing of all the discharging switches during the output signal creation in a period of time of $2 \mathrm{~ms}$.

- the Flattener Driving System (FDS) that is used in order to fire, with an appropriate time control sequence, the charging and discharging switches of the Flattener circuit in order to open and close sequentially the switches for the Main Marx droop compensation in the high voltage output pulses.

At the end there is a need to develop the fourth baseline component that is the Klystron Energy Drop-Out Safety Systems (KEDOSS) that will allow the protection from arcs, when the klystron faults, by suddenly quenching the klystron arc into a discharge of the modulator stored energy. Finally, in the present work we have proved, at a simulation level that $\mathrm{SM}^{2}$ fits all the ILC TDR expectations even after a severe Montecarlo simulation of the circuit behavior. 


\section{Appendix A}

\section{A.1 The synchrotron radiation energy loss}

If a charge is accelerated but is observed in a reference frame where its velocity is small compared to that of light, the instantaneous energy flux is given by the Poynting's vector,

$$
S=\frac{c}{4 \pi} E \times B=\frac{c}{4 \pi}\left|E_{a}\right|^{2} n
$$

This means that the power radiated per unit solid angle is

$$
\frac{d P}{d \Omega}=\frac{c}{4 \pi}\left|R E_{a}\right|^{2}=\frac{e^{2}}{4 \pi c} \mid n \times\left(n \times\left.\dot{\beta}\right|^{2}\right.
$$

If $\Theta$ is the angle between the acceleration $v$ and $n$ then the power radiated can be written

$$
\frac{d P}{d \Omega}=\frac{e^{2}}{4 \pi c^{3}} \dot{v}^{2} \sin ^{2} \Theta
$$

This exhibits the characteristic $\sin ^{2} \Theta$ angular dependence which is a well know result. The total instantaneous power radiated is obtained by integrating (A.3) over all solid angle. Thus

$$
P=\frac{2}{3} \frac{e^{2} \dot{v}^{2}}{c^{3}}
$$

This is the familiar Larmor result for a non relativistic, accelerated charge. Larmor's formula (A.4) can be generalized by arguments about covariance under Lorentz transformations to yield a result which is valid for arbitrary velocities of the charge. Radiated electromagnetic energy behaves under Lorentz transformation like the fourth component of a 4-vector. Since $d E_{\text {rad }}=P d t$, this means that the power $\mathrm{P}$ is a Lorentz invariant quantity. If we can find a Lorentz invariant which reduces to the Larmor formula (A.4) for $\beta<<1$, then we have the desired generalization. There are, of course, many Lorentz invariant which reduce the desired form when $\beta \rightarrow 0$. For an appropriate generalization we write Larmor's formula in the suggestive form:

$$
P=\frac{2}{3} \frac{e^{2}}{m^{2} c^{3}}\left(\frac{d p}{d t} \cdot \frac{d p}{d t}\right)
$$

where $m$ is the mass of the charge, and $p$ its momentum. The Lorentz invariant generalization is clearly

$$
P=\frac{2}{3} \frac{e^{2}}{m^{2} c^{3}}\left(\frac{d p_{\mu}}{d \tau} \frac{d p_{\mu}}{d \tau}\right)
$$

where $d \tau=d t / \gamma$ is the proper time element , and $p_{\mu}$ is the charged particle's momentumenergy 4-vector. To check that (A.6) reduces properly to (A.5) as $\beta \rightarrow 0$ we evaluate the 4vector scalar product,

$$
\frac{d p_{\mu}}{d \tau} \frac{d p_{\mu}}{d \tau}=\left(\frac{d p}{d \tau}\right)^{2}-\frac{1}{c^{2}}\left(\frac{d E}{d \tau}\right)^{2}=\left(\frac{d p}{d \tau}\right)^{2}-\beta^{2}\left(\frac{d p}{d \tau}\right)^{2}
$$


Radiation losses are sometimes the limiting factor in the maximum practical energy attainable. For a given applied force the radiated (A.6) depends inversely on the square of the mass of the particle involved. Consequently these radioactive effects are largest for electrons. So in a linear accelerator the motion is one dimensional. From (A.7) it is evident that in the that case the radiated power is

$$
P=\frac{2}{3} \frac{e^{2}}{m^{2} c^{3}}\left(\frac{d p}{d t}\right)^{2}
$$

The rate of change of momentum is equal to the change in energy of the particle per unit distance. Consequently

$$
P=\frac{2}{3} \frac{e^{2}}{m^{2} c^{3}}\left(\frac{d E}{d x}\right)^{2}
$$

showing that for linear motion the power radiated depends only on the external forces which determine the rate of change of particle energy with distance, not on the actual or momentum of the particle. The ratio of power radiated to power supplied by the external sources is

$$
G=\frac{P}{d E / d x}=\frac{2}{3} \frac{e^{2}}{m^{2} c^{3}} \frac{1}{v} \frac{d E}{d x} \rightarrow \frac{2}{3} \frac{\left(e^{2} / m c^{2}\right)}{m c^{2}} \frac{d E}{d x}
$$

Where the last form holds for relativistic particles $(\beta \rightarrow 1)$. Equation (A.10) shows that the radiation loss will be unimportant unless the gain in energy is of the order $m c^{2}=0.511 \mathrm{MeV}$ in a distance of $e^{2} / \mathrm{mc}^{2}=2.82 * 10^{-13} \mathrm{~cm}$, or of the order of $2 * 10^{14} \mathrm{MeV} / \mathrm{meter}$. Typical energy gains less than $10 \mathrm{MeV/meter.} \mathrm{In} \mathrm{fact} \mathrm{if} \mathrm{we} \mathrm{considered} \mathrm{a} \mathrm{gain} \mathrm{G}=1$ this is the case in which total energy that receive the particle is eliminated as radiation and so

$$
\left(\frac{d E}{d x}\right)=\frac{3}{2} \frac{m c^{2}}{2.8 * 10^{-13} \mathrm{~cm}}=2.5 * 10^{14} \mathrm{MeV} / \mathrm{m}
$$

In the ILC, the $(\mathrm{dE} / \mathrm{dx})=31.5 \mathrm{MeV} / \mathrm{m}$ and so the beam losses are unimportant. Moreover in the ILC the gain is

$$
G \approx \frac{2}{3} \frac{2.8 * 10^{-13} \mathrm{~cm}}{0.51 \mathrm{MeV}} 31.5 \mathrm{MeV} / \mathrm{m} \approx 1.15 * 10^{17}
$$

Circumstances change drastically in circular accelerators like the synchrotron or betatron. In such machines the momentum $p$ changes rapidly in direction as the particle rotates, but the change in energy per revolution is small. Then the radiated power (A.10) can be written approximately

$$
P=\frac{2}{3} \frac{e^{2}}{m^{2} c^{3}} \gamma^{2} \omega^{2}|p|^{2}=\frac{2}{3} \frac{e^{2} c}{\rho^{2}} \beta^{4} \gamma^{4}
$$

Where we have used $a=(c \beta / \rho), \rho$ being the orbit radius. This result was first obtained by Liènard in 1898. The radioactive-energy loss per evolution is

$$
\delta E=\frac{2 \pi \rho}{c \beta} P=\frac{4 \pi}{3} \frac{e^{2}}{\rho} \beta^{3} \gamma^{4}
$$


For high-energy electrons $((\beta \approx 1)$ this has the numerical value

$$
\delta E(M e V)=8.85 * 10^{-2} \frac{[E(\text { Bev })]^{4}}{\rho(\text { meter } s)}
$$

For a typical low-energy synchrotron, $\rho \approx 1$ meter, $\mathrm{E}_{\max } \sim 0.3 \mathrm{BeV}$. Hence, $\delta \mathrm{E}_{\max } \sim 1 \mathrm{KeV}$ per revolution. This is less than, but not negligible compared to, the energy gain of a few kilovolts per turn. In the largest electron synchrotron, the orbit radius is the order of 10 meters and the maximum energy is $5 \mathrm{BeV}$. Then the radioactive loss is about $5.5 \mathrm{MeV}$ per revolution. Since it extremely difficult to generate radiofrequency power at levels high enough to produce energy increments much greater than this amount per revolution, it appears that $5-10 \mathrm{BeV}$ is an upper limit on the maximum energy of circular electrons accelerators. The power radiated in circular accelerators can be expressed numerically as

$$
P(\text { watts })=\frac{10^{6}}{2 \pi} \frac{\delta E(\mathrm{MeV})}{\rho(\text { meters })} J(\text { amp })
$$

where $\mathbf{J}$ is the circulating beam current. This equation is valid if the emission of radiation from the different electrons in the circulating beam is incoherent. In the largest electron synchrotron the radiated power amounts to 0.1 watt per microampere of beam.

For an accelerated charge in nonrelativistic motion the angular distribution shows a simple $\sin ^{2} \Theta$ behavior, as given by (A.3), where $\Theta$ is measured relative to the direction of acceleration. For relativistic motion the acceleration fields depend on the velocity as well as the acceleration and consequently the angular distribution is more complicated. I we imagine a linear motion of charge during which the ordinary velocity and acceleration have same magnitude, direction and are parallel so the power radiated per unit solid angle, proportional to the angular distribution of the energy radiated is

$$
\frac{d P\left(t^{\prime}\right)}{d \Omega}=\frac{e^{2} \dot{v}^{2}}{4 \pi c^{3}} \frac{\sin ^{2} \theta}{(1-\beta \cos \theta)^{5}}
$$

For $\beta<<1$, this is the Larmor result (A.4). But as $\beta \rightarrow 1$, the angular distribution is tipped forward more and more increases in magnitude. The angle $\Theta_{\max }$ for which the intensity is a maximum is

$$
\theta_{\max }=\cos ^{-1}\left[\frac{1}{3 \beta}\left(\sqrt{1+15 \beta^{2}}-1\right)\right] \rightarrow \frac{1}{2 \gamma}
$$

Where the last form is the limiting value for $\beta \rightarrow 1$. In this same limit the peak intensity is proportional to $\gamma^{8}$. Even for $\beta=0.5$, corresponding to electrons of $\sim 80 \mathrm{KeV}$ kinetic energy, $\Theta_{\max }=38.2^{\circ}$. For relativistic particle, $\Theta_{\max }$ is very small, being of the order of the ratio of the rest energy of the particles to its total energy. Thus the angular distribution is confined to a very narrow cone in the direction of motion. For such small angles the angular distribution (A.17) can be approximately

$$
\frac{d P\left(t^{\prime}\right)}{d \Omega} \approx \frac{8}{\pi} \frac{e^{2} \dot{v}^{2}}{c^{3}} \gamma^{8} \frac{(\gamma \theta)^{2}}{\left(1+\gamma^{2} \theta^{2}\right)^{5}}
$$



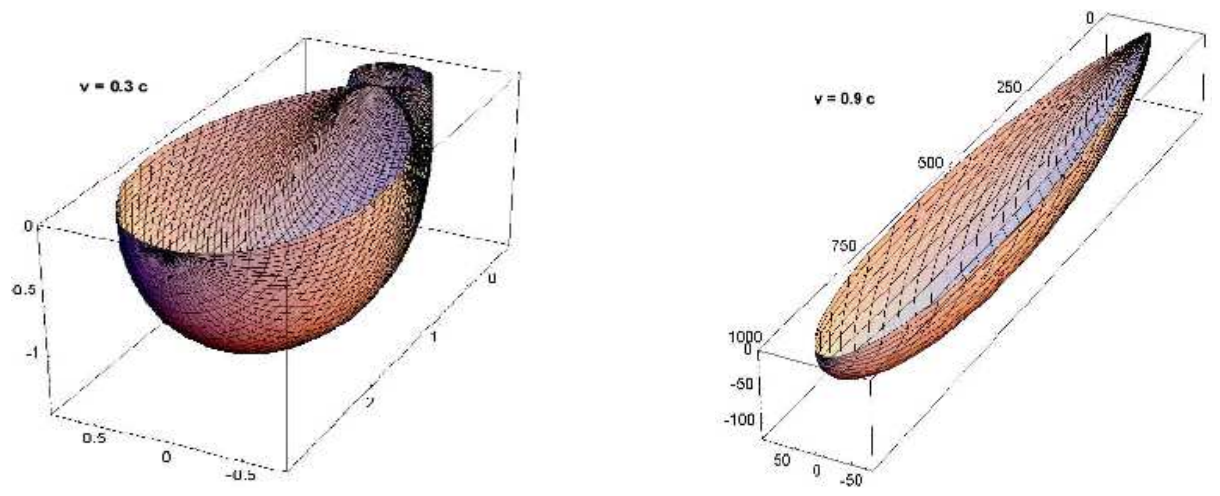

Figura A.1: Angular distribution of synchrotron radiation

The natural unit is evidently $\gamma^{-1}$. The peak in the angular distribution occurs at $\gamma \theta=\frac{1}{2}$ and the half-power points at $\gamma \theta=0.23$ and $\gamma \theta=0.91$. The root mean square angle of emission of radiation in the relativistic limit is

$$
\langle\theta\rangle^{\frac{1}{2}}=\frac{1}{\gamma}=\frac{m c^{2}}{E}
$$

The total power radiated can be obtained by integrating (A.17) over all angles. Thus

$$
P\left(t^{\prime}\right)=\frac{2}{3} \frac{e^{2}}{c^{3}} \dot{v}^{2} \gamma^{6}
$$

in agreement with (A.18).

Another example of angular distribution of radiation is that for a charge instantaneously circular motions with its acceleration perpendicular its velocity. At this point we choose a coordinate system such that instantaneously velocity in the $z$ direction and acceleration in the $x$ direction. With the customary polar angles $\Theta, \Phi$ defining the direction of observation, the formula is

$$
\frac{d P\left(t^{\prime}\right)}{d \Omega}=\frac{e^{2} \dot{v}^{2}}{4 \pi c^{3}} \frac{1}{(1-\beta \cos \theta)^{3}}\left[1-\frac{\sin ^{2} \theta \cos ^{2} \phi}{\gamma^{2}(1-\beta \cos \theta)^{2}}\right]
$$

We note that, although the detailed angular distribution is different from the linear acceleration case, the same characteristic relativistic peaking at forward angles is present .In the relativistic limit $(\gamma>>1)$, the angular distribution can be written approximately

$$
\frac{d P\left(t^{\prime}\right)}{d t} \approx \frac{2}{\pi} \frac{e^{2} \dot{v}^{2}}{c^{3}} \gamma^{6} \frac{1}{\left(1+\gamma^{2} \theta^{2}\right)^{3}}\left[1-\frac{4 \gamma^{2} \theta^{2} \cos ^{2} \phi}{\left(1+\gamma^{2} \theta^{2}\right)^{2}}\right]
$$


The root mean square angle of emission in this approximation is given by (A.20), just as for one-dimensional motion. The total power radiated can be found by integrating (A.22):

$$
P\left(t^{\prime}\right)=\frac{2}{3} \frac{e^{2} \dot{v}^{2}}{c^{3}} \gamma^{4}
$$

It is instructive to compare the power radiated for acceleration parallel to the velocity (A.23) or (A.8) with the power radiated for acceleration perpendicular to the velocity (A.4) for the same magnitude of applied force. For circular motion, the magnitude of the rate of change of momentum (which is equal to the applied force) is $\gamma m \dot{v}$. Consequently, (A.24) can be written

$$
P_{\text {circular }}\left(t^{\prime}\right)=\frac{2}{3} \frac{e^{2}}{m^{2} c^{3}} \gamma^{2}\left(\frac{d p}{d t}\right)^{2}
$$

When this is compared to the corresponding result (A.8) for rectilinear motion, we find that for a given magnitude of applied force the radiation emitted with a transverse acceleration is a factor of $\gamma^{2}$ larger than with a parallel acceleration. 


\section{Appendix B}

\section{B.1 Collider luminosity}

The event rate $\mathrm{R}$ in a collider is proportional to the interaction cross section $\sigma_{\text {int }}$ and the factor of proportionality is called the luminosity:

$$
R=L \sigma_{\text {int }}
$$

It two bunches containing $n_{1}$ and $n_{2}$ particles collide with frequency $f$, the luminosity is:

$$
L=f \frac{n_{1} n_{2}}{4 \pi \sigma_{x} \sigma_{y}}
$$

where $\sigma_{x}$ and $\sigma_{y}$ characterized the Gaussian transverse beam profiles in the horizontal and vertical directions and to simplify the expression it is assumed that the bunches are identical in transverse profile, thet the profiles are independent of position along the bunch, and the particle distributions are not altered during collision. Whatever the distribution at the source, by the time the beam reaches high energy, the normal form is good approximation thanks to the central limit theorem of probability ant the diminished importance of space charge effects. The beam size can be expressed in terms of two quantities, one termed the transverse emittante, $\epsilon$, and the other, the amplitude function $\beta$. The transverse emittance is a beam quality concept reflecting the process of bunch preparation, extending all the way back to the source for hadrons and, in the case of electrons, mostly dependent on synchrotron radiation. The amplitude function is a beam optics quantity and is determined by the accelerator magnet configuration. When expressed in terms of $\sigma$ and $\beta$ the transverse emittance becomes

$$
\varepsilon=\pi \sigma^{2} / \beta
$$

Of particular significance is the value of the amplitude function at the interaction point, $\beta^{*}$. Clearly one wants $\beta^{*}$ to be as small as possible; how small depends on the capability of the hardware to make a near-focus at the interaction point.

Eq. (B.2) can now be recast in terms of emittances and amplitude function as

$$
L=f \frac{n_{1} n_{2}}{4 \sqrt{\varepsilon_{x} \beta^{*}{ }_{x} \varepsilon_{y} \beta^{*}{ }_{y}}}
$$

Thus to achieve high luminosity, all one has to do is make high population bunches of low emittance to collide at high frequency at locations where the beam optics provides as low values of the amplitude functions as possible. 


\section{Bibliography}

[1] R. D. Heuer, D. Miller, F. Richard, P. Zerwas (Editors), "TESLA Technical Design Report, Part III', http://tesla.desy.de/new pages/TDR CD/start.html;

[2] D. N. Spergel et al., arXiv:astro-ph/0603449;

[3] A. Birkedal, K. Matchev and M. Perelstein, Phys. Rev. D 70 (2004) 077701;

[4] J. Feng, "ILC Cosmology”, arXiv:hep-ph/0509309;

[5] C. Pagliarone, L. Diaz-Cruz, "Perspectives of detecting CKM-suppressed top quark decays at ILC" Proceedings of Summer School and Conference on New Trends in HighEnergy Physics: Experiment, Phenomenology, Theory, Yalta, Crimea, Ukraine, 16-23 Sep 2006. e-Print: hep-ph/0612120;

[6] C.Pagliarone, L. Diaz-Cruz, R. Gaitan-Lozano, G. Lopez-Castro, "CKM-suppressed top quark decays $t \rightarrow s(d)+W$ in the standard model and beyond”, Phys.Rev.D77:094010,2008;

[7] C.Pagliarone, "Extra dimensions and black hole production", Proceedings of International Conference on New Trends in High-Energy Physics: Experiment, Phenomenology, Theory, Yalta, Crimea, 22-29 Sep 2001. hep-ex/0.111063;

[8] V. Vogel and S. Choroba, "Status Toshiba MB", (2006);

[9] E. Wright, H. Bolen, S. Lenci, and A. Balkcum, "RF Source Selection for the ILC", Snowmass 2005 (2005);

[10] D. Sprehn, "MBK Testing at DESY - May 22 -June 2 2006", (2006);

[11] A. Beunas, G. Faillon, S. Choroba, A. Gamp, "A High Efficiency Long Pulse Multi Beam Klystron for the Tesla Linear Collider", PAC01 (2001);

[12] H.-J. Eckoldt, N. Heidbrook, "Power Supplies for Modulators", DESY print. TESLA 2000-36;

[13] F. Belloni, P.Maranesi, M. Riva, "DC to DC Converters for the International Space Station" proceedings of the EPE Conference '03, Sep.2003;

[14] M. Legnani, P.G. Maranesi, G. Naummi, "SILC: a Novel Phase-Shifted PWM Power Converter", Proceedings of the EPE '93, Vol.3;

[15] J.A.Sabatè, V. Vlatkovic, R.B. Ridley, F.C. Lee, B.H. Cho, "Design Consideration for High-Voltage High-power Full Bridge Zero-Voltage-Switched PWM Converter", Proceedings of the IEEE APEC '90,pp. 275-284;

[16] Tingting Song, Huang N., Ioinovici A., "A zero-voltage and zero-current switching three-level DC-DC converter with secondary assisted generative passive snubber", Circuits and Systems, 2004. ISCAS '04. Proceedings of the 2004 International Symposium on, Volume 5, 23-26 May 2004;

[17] Ned Mohan,William P. Robbins, "Power Electronics: Converters and Applications" ; [18] http://www.datasheetcatalog.org/datasheet/MitsubishiElectricCorporation/mXvz.pdf;

[19] Z. Ghasemi, S. Macgregor, J. Andreson, and Y. Lamont. "Development of an integrated solid-state generator for light inactivation of food-related pathogenic bacteria”. Measurement Science and Technology, 14(6):26-32, 2003; 
[20] K.H. Schoenbach, R.H. Stark, S. Beebe, and S. Buescher. "Bioelectrics - new applications for pulsed power technology". IEEE International Conference on Plasma Science, pages 293-300, 2001;

[21] M.I. Bazhal, N.I. Lebovka, and E. Vorobiev. "Pulsed electric field treatment of apple tissue during compression for juice extraction”. Journal of Food Engineering, 50(3):129-139, 2001;

[22] Geun-Hie Rim, Chu-Hyun Cho, Hong-Silk Lee, and E.P. Pavlov."Electric-blast system for rock fragmentation”. Digest of Technical Papers-IEEE International Pulsed POWER Conference, 1:165-168, 1999;

[23] Ronald J. Kaye, Bob N. Turman, and Steven L. Shope. "Applications of coil gun electromagnetic propulsion technology”. IEEE Transactions on Plasma Science, 25(2):155159, 1997;

[24] M.P.J. Gaudreau, J.A.Casey, M.A.Kempkes, T.J.Hawkey, and J.M.Mulvaney "Solidstate modulators for plasma immersion ion implantation applications", J. Vac. Sci. Technol. B, Microelectron. Nanometer Struct. (USA), 17(2):888-894, 1999;

[25] R.J. Ritcher-Sand, R.J. Adler, R. Finch, and B. Ashcraft. "Marx-stacked IGBT modulators for high voltage, high power applications". IEEE Conference Record of power Modulator Symposium, pages 390-393, 2002;

[26] H. Pfeffer, L.Bartelson, K.Bourkland, "A Long Pulse Modulator for Reduced Size and Cost”- Proc. of Fourth European Particle Accelerator Conference. London, 1994;

[27] H. Pfeffer, L.Bartelson, K.Bourkland, "A Second Long Pulse Modulator for Reduce Size and Cost"- Proceedings of Fifth European Particle Accelerator Conference. Barcelona, 1996; [28] H. Pfeffer, L.Bartelson, K. Bourkland, C.Jensen, Q. Kerns, P. Prieto, G. Saewert, D.Wolff, "THE TESLA 5 MEGAWATT MODULATOR”;

[29] H. Pfeffer, C. Jensen \& D. Wolff, "The FERMI SMTF Modulators", (2005);

[30] C. Jensen, H. Pfeffer, D. Wolff, “ILC Modulator Talk”, (2004).

[31] “International Linear Collider TDR”, August 2007. 
This document was created with Win2PDF available at http://www.win2pdf.com. The unregistered version of Win2PDF is for evaluation or non-commercial use only. This page will not be added after purchasing Win2PDF. 